\title{
SAME BUT DIFFERENT: Chaos and TV Drama Narratives
}

\author{
BY
}

\author{
ALISON MCLACHLAN
}

A thesis submitted to the Victoria University of Wellington in fulfilment of the requirements for the degree of Doctor of Philosophy

Victoria University of Wellington

(2019) 

Complexity is a term that is now commonly used when discussing TV serial dramas and the way that, in recent years, creators and producers of this narrative form have embraced innovative and challenging strategies to tell their stories. As a result, it is also often argued that all TV serial dramas are strikingly different from one another; one of the few things that contemporary TV serial dramas have in common is their employment of complex narrative strategies. However, in this thesis, I argue that-while serial dramas are different from one another in many ways - they are also all the same at a fundamental level.

In order to examine the fundamental narrative components that all serial dramas employ, I use chaos as a framework. Chaos is a branch of mathematics and science which examines systems that display unpredictable behaviour that is actually determined by deep structures of order and stability. At its most basic level, chaos corresponds with the way in which serial dramas are both complex and simple at the same time; beneath the complexity of serial dramas are fundamental building blocks that are used to generate innovative, challenging and unpredictable narratives.

I apply the findings from my critical examination of chaos and TV drama narratives to the creation of my own TV projects, which employ the inherent structures and patterns of TV drama narratives in a way that produces innovative and complex stories. In doing so, I intend to highlight the potential of serial dramas to be endlessly creative yet consistently the same. 



\section{ACKNOWLEDGEMENTS}

I would like to thank everyone at the IIML-especially Damien Wilkins, Katie HardwickSmith, and Clare Moleta-for providing the time, space and support that made this project possible. Many thanks also go to my fellow PhD creative writing cohort for their feedback and support: Angela Andrews, Valerie Arvidson, Airini Beautrais, Peter Cox, Amy Leigh Wicks, Allan Drew, Kate Duignan, Gigi Fenster, Helen Heath, Therese Lloyd, Monica Macanstantos, Sue Orr, Anna Sanderson, Claire Baylis, Nikki-Lee Birdsey, Ben Egerton, Miles Fuller, Helen Innes, Justine Jungersen-Smith, Mikaela Nyman, and Lena Tichy.

I would also like to thank my mum, my dad, Dougal McLachlan, Hamish McLachlan, GARAR, and Gavin McGibbon for shaping me and helping, in various capacities, to get me to where I am.

My immense gratitude also goes to Megan Le Bas, Kim Le Bas, Florence Le Bas, Lois Le Bas, Diane Owen, Doug Owen, and Kirsty Owen, for welcoming me into your family and providing encouragement, support and countless getaways.

Special thanks go to:

Fiona McLachlan for being an amazing sister and role model.

Fiona Druitt for sharing your knowledge of chaos, and providing such generous and in-depth feedback.

Cameron McLachlan for your rigorous proof-reading and endless support.

My secondary supervisor, Joost de Bruin, for your valuable feedback and calming reassurance.

My primary supervisor, Ken Duncum, for your insights, gentle encouragement, and general wizardry.

My deepest gratitude is for René Le Bas. This thesis wouldn't exist without your love, encouragement, patience, tolerance, and humour. I'm very lucky to have you. 



\section{PART ONE: CRITICAL COMPONENT}

INTRODUCTION

Complexity in TV Drama Narratives

Methodology and Thesis Outline

CHAPTER ONE: “Universality" and the Narrative Structures of TV Dramas

Introduction

"Universality"

24

"Universality" and the Narrative Structures of TV Dramas 29

"Universality" and The Wire 41

Conclusion

CHAPTER TWO: Strange Attractors and Unpredictability in TV Drama Narratives

Introduction

54

Strange Attractors

55

Strange Attractors and Unpredictability in TV Drama Narratives

\section{PART TWO: CREATIVE COMPONENT}

INTRODUCTION

Untold: Series Bible

Untold: Episode Synopses

Untold: Episode One script

Untold: Episode Two script 
Bad News: Series Bible

Bad News: Episode synopses

Bad News Scripts: Episode One script

Bad News: Episode Two script

Bad News and Chaos

PROJECT THREE: THE CHANCES

The Chances: Series Bible

The Chances: Episode Synopses

The Chances Scripts: Episode One script

The Chances: Episode Two script

376

The Chances and Chaos

CONCLUSION

429

BIBLIOGRAPHY

433 


\section{PART ONE: \\ CRITICAL COMPONENT}





\section{INTRODUCTION}

When water is poured into a waterwheel that has holes at the bottom of each of its buckets, the behaviour of the wheel will be unpredictable. This is because the weight of each bucket depends on how much water it contains, as well as how much water it lets out. But the amount of water that a bucket leaks out also depends on how much water it initially contains. This interlinked relationship between how much water is going in and out of each bucket at one time means that, if the flow of water from the top is fast enough, the wheel will display both regular and irregular behaviour. An observer can never predict when the wheel will perform regular or irregular behaviour because of the infinitesimally tight intertwining of stability and instability in the system. This particular waterwheel is known as the Lorenz Waterwheel, named after Edward Lorenz, an applied mathematician and meteorologist who is often considered the father of chaos*-an area of mathematics that examines instability in nonlinear dynamical systems (Gleick 29).

Chaos is commonly associated, by non-mathematicians, with "the butterfly effect"; a term coined after an image that Lorenz came up with to describe chaos, also known as sensitive dependence on initial conditions. That is, the flap of a butterfly's wings can cause a tornado on the other side of the world. This image captures the essence of chaos-that minor perturbations in a chaotic system, like the weather, can have catastrophic and unpredictable consequences in the future due to the infinitesimally tight intertwining of stability and instability. In other words, the weather and the Lorenz Waterwheel are both systems that perform chaos because their behaviour is unpredictable yet deterministic (a system's current state determines the next state). For instance, the speed at which the water flows into the Lorenz Waterwheel initially determines the rate at which the first bucket will fill and leak, which will in turn determine the speed at which the wheel rotates, which in turn determines the rate at which each subsequent bucket will fill and leak, and so on. In this sense, its current state determines its future state but, if the flow of water

\footnotetext{
*The study of nonlinear dynamical systems is popularly known as "chaos theory" in the humanities, but within the field itself is more commonly referred to as "chaos" - I will use the term "chaos" throughout this thesis.
} 
reaches a certain speed, it is not possible to predict what these future states will be-it is impossible to know what the wheel will do next at any given point. Thus, a chaotic system displays behaviour that is determined by particular conditions and states yet is unpredictable (Gleick 27).

In this thesis I argue that the narratives in critically successful TV serial dramas* resemble the behaviour of a chaotic system, like that of the Lorenz Waterwheel. I will also argue that the creators of successful TV serial dramas are attempting, albeit inadvertently, to produce storylines that resemble the chaotic behaviour of these systems. I see this resemblance as apparent in the way that all successful serial dramas are underpinned by the same basic narrative structures, or building blocks, that are utilised in ways that produce unique and engaging stories. In other words, for a serial drama to be successful it must continually strike the difficult balance of being rigidly structured yet endlessly creative; familiar and expected yet surprising and unpredictable; the same but different.

Before I examine serial drama narratives through this lens of chaos in more depth, I will provide a general overview of how scholars and critics have perceived complexity in relation to TV drama. I will then provide a brief introduction to chaos, followed by an examination of how chaos has been successfully and not-so-successfully adopted by those in the field of humanities. From there I will outline my own method of comparing TV serial narratives to chaos and how this will form chapters one and two of this thesis. I will then go on to introduce the three TV projects that I have written as part of my creative component, which have simultaneously informed and been informed by the critical component of this thesis.

\section{Complexity in TV Drama Narratives}

The past two decades have seen a distinct increase in the production of compelling, challenging and risk-taking serial TV dramas, which has in turn given rise to the term "complex TV" in the academic field of television studies. Two major scholars who have investigated "complex TV" in depth - Trisha Dunleavy and Jason Mittell - argue that

\footnotetext{
*While I am aware there are different types of "success" in serial dramas (such as commercial success), I will use "critically successful" serial dramas as the focus of this thesis.
} 
complexity in TV emerged in the early 2000s and has since continued to develop and evolve as technology and viewing platforms have changed (Mittell 1). Dunleavy's book, Complex Serial Drama and Multiplatform Television, examines the authorship processes, narrative characteristics, industrial practices and stylistic tendencies of American 'complex serial' drama. Throughout her book, Dunleavy describes several different characteristics of complex serials, most of which can be identified when comparing and contrasting the narrative form to the conventions and traditions of broadcast television. She posits that complex dramas tend to be more sophisticated and challenging than more traditional TV narratives; complex dramas construct a detailed narrative memory, and are not designed to be viewed casually but rather require and reward audiences who pay close attention (3). This "sophistication" is a result of complex TV dramas being grounded in the serial form rather than the series form; complex TV dramas employ a single, overarching story that spans all episodes, "requiring narrative progression, unavoidable change and an inevitable end", whereas the storylines in a series are generally developed and resolved within the space of an individual episode (I will discuss these two forms in more detail below) (165). Dunleavy also explains that the concepts or premises of complex serial dramas favour unique settings and milieux, compared to the TV drama tradition of employing tried-andtrue institutions like police stations and hospitals to generate story. The favouring of unique settings allows the narratives of complex dramas to explore conflict-driven and transgressive characters as opposed to investigating situations that present conflict for the characters (105). Another characteristic of complex serial dramas that Dunleavy identifies is the use of "additional scenes" like flashbacks and flashforwards to add depth to the tapestry of the characters and their stories (105). Dunleavy writes that these techniques and strategies of complex serial dramas have come about because of the shift into "multiplatform television", such as IDTV (internet-distributed TV), which has meant that TV drama narratives are no longer limited to linear schedules of broadcast TV, and that these particular shows are often more lucrative in terms of their afterlife, rather than when they air (10-11).

Like Dunleavy, Mittell also sees the diverse strategies that serial dramas employ, as well as the different ways in which audiences can watch and consume these serials, as being an indicator of the narrative form's complexity. In his book, Complex TV: The Poetics of 
Contemporary Television Storytelling, Mittell outlines what he deems to be the main strategies that creators of complex TV employ: utilising serial techniques, playing with temporality, constructing ongoing characters, and incorporating transmedia (10). As well as outlining the different strategies of complex TV, Mittell also briefly acknowledges that there are some formal elements and narrative patterns, which emerged out of the constraints of broadcast TV, that all serial dramas continue to borrow and build off. Mittell suggests that it is the way in which the creators of serial dramas employ these elements and patterns in different and nuanced ways that leads to complex storytelling in TV (4). Mittell writes that his emphasis on complex TV narratives, "[...] is not to downplay the importance of traditional pleasures of character depth, neat resolution of plots, storyworld consistency, action-oriented excitement, and humor-complex television at its best employs all of these elements while adding the operational possibilities of formal engagement" (53). According to Mittell, these "operational possibilities of formal engagement" are evident in the way that complex TV drama narratives often challenge viewers by playing with their expectations, inviting temporary confusion and disorientation, and requiring viewers to develop their comprehension skills through long-term viewing and active engagement (261). And so, while their approaches and terminology are slightly different, both Mittell and Dunleavy posit that complex serial dramas are all very different on a conceptual level, but that the one main aspect that unifies them is that they all work against convention in intriguing ways; these vastly different shows can be grouped together because of their shared deployment of complex seriality (Mittell 18; Dunleavy 121).

As is evidenced in Mittell and Dunleavy's work, the nature of seriality is a topic that appears in many studies on TV drama narratives, even in works that aren't explicitly discussing the recent development of TV's complexity. As I mentioned earlier, a series is generally categorised as an episodic TV show in which the storylines are contained within single episodes. A procedural drama, for instance, will have a "case of the week" format, which means that episodes have their own stand-alone plots that are introduced and resolved within the same episode. As a result, a viewer does not need to have seen previous episodes in order to follow the story. At the other end of the spectrum is the serial, which has openended episodes that contribute to an overarching, or continuous, storyline. A viewer needs to have seen previous episodes of a serial in order to follow the story. Mittell writes, "When 
we talk about a serialized program, we are usually referring less to the ubiquitous persistence of storyworld and characters and more to the ongoing accumulation of narrative events-what occurs in one episode will have happened to the characters and storyworld as portrayed in future episodes" (22-23).

While complex TV drama narratives tend to exist on the serialised end of the spectrum, Jeremy Butler argues that most TV drama narratives are a mixture of both forms, and suggests that it is difficult to tell the two forms apart-even though a TV show will usually be categorised as one or the other (ctd. in Gil 179). Kirstin Thompson posits that a certain degree of continuation, but not continuation itself, is the main focus in determining a series from a serial (101). Thompson's claim is similar to that put forward by Dunleavy who goes into more detail about the varying degrees of continuing narratives in TV drama, suggesting that some shows could be considered a "series-serial" or a "serialised series" $(155,158)$. Glen Creeber claims that a series is usually never ending, but involves self-contained episodes, whereas a serial follows an unfolding, episodic narrative structure that moves progressively towards a conclusion (8). The term "flexi-narrative", coined by Robin Nelson, is often adopted in these discussions about how the boundary between serial and series is merging. He defines the term as a "hybrid mix of serial and series forms [...] involving the closure of one story arc within an episode (like a series) but with other, ongoing story arcs involving the regular characters (like a serial)" (82). Despite these slight variations in the understanding of the distinctions of series and serials, it is clear that all successful complex serial dramas, at some level, are a combination of open and closed structures; even if a serial drama mostly consists of ongoing storylines, its narrative will also contain smaller narratives within it. And so, while this thesis will be focusing on the narrative form of TV serial dramas, it will-as a result of this hybridity-also be examining the conventions of series that the complex serial borrows from.

In particular, this combination of open and close narratives in a complex serial is due to the way in which a TV drama narrative is constructed of various parts or units. Sometimes these "parts" are the same as the smaller, closed-off narratives that exist within the larger ongoing narrative but, beyond this, are the more rigid narrative units that constitute a TV series or serial as a whole. That is, a TV show is made up of seasons, which are made up of 
episodes, which are each made up of act breaks, which are each made up of scenes. Many have put forward similar arguments about the various parts that make up a TV drama narrative but give these parts different names and explanations. Dunleavy suggests, in her discussion of "segmentation", that the narrative conventions and forms of TV drama have developed out of a need to accommodate the interruptions of commercial breaks. Michael Newman claims that TV narratives can be thought of as a process of accumulating information, and that TV writers aim to parcel out this information into what he identifies as the beat, the episode, and multi-episode arcs (17). Newman claims that parcelling the narrative out like this achieves "[...] a sense of proportion and symmetry, ensures a steadily rising action, and organises patterns of attention and expectation" (21). The main units of TV narratives according to Porter et al. are what Seymour Chatman calls kernels and satellites. If a kernel were altered, the plot would be changed and the entire story would be different, whereas a satellite focuses on character, setting, or incidental actions that do not move the story along its causal trajectory (25). In Finding Meaning in All the Right Places: A Novel Measurement of Dramatic Structure in Film and Television Narratives, Coleman et al. coin the term "breakpoint" to describe when one action appears to begin or end in TV narratives, and they argue that these breakpoints inevitably coincide with "meaning moments" in the narrative. They conclude that breakpoints in TV enable comprehensibility, and that the overall narrative is deemed by the audience to be less important than the specific moments within the overall narrative that mark distinct changes in the story (30).

Whether they are called signs, texts, units, beats, arcs, break-points, satellites, kernels, or segments, these distinctive structural narrative components that make up TV drama narratives have largely formed in response to commercial constraints-imposed timeframes, ad breaks, the need for cliff-hangers. But these various components have been incorporated, and are now embedded, into the narrative form in innovative ways that enable the rise and fall of action, provide regular turning points, and allow for variety within the overarching story of a TV serial.

And so, just as Mittell suggests that, despite the recent innovations and developments of complex TV drama narratives, serials continue to incorporate traditional narrative strategies such as "character depth, neat resolution of plots, storyworld consistency, action-oriented 
excitement, and humor", this thesis will be examining how, despite its innovation and developments, the complex serial form continues to utilise these various parts, structures and patterns to tell its stories (53). In other words, while scholarship that examines TV's complexity mostly focuses on how all serial dramas are different from one another, this thesis will focus on the ways in which serial dramas are the same. This is not to say that all serial dramas are exactly the same, or that these differences that other scholars have identified don't exist, but rather that all serial dramas utilise the same fundamental building blocks to produce narratives that are different from one another; serial dramas employ the same essential structures to produce innovative, challenging and complex stories. I will be using chaos as a lens through which to examine how serial dramas can be both simple and complex-the same but different. Below is a brief introduction to chaos, and why it is a useful tool with which to examine the similarities of all complex serial dramas.

\section{Introduction to Chaos}

In Chaos: The Amazing Science of the Unpredictable, James Gleick largely attributes the emergence of chaos to Edward Lorenz's exploration of sensitivity to initial conditions in the 1960s (16). Gleick explains that, in an attempt to predict the weather more than a few days in advance, Lorenz produced twelve differential equations that captured the phenomena of the weather: the interconnected causes and effects of atmospheric variables such as heat, moisture, temperature, wind speed, and air pressure. Lorenz programmed his computer to solve his differential equations and found that the mathematical patterns and sequences being produced by the computer were not dissimilar to the real weather patterns that were occurring outside his window. Lorenz, wanting to predict the weather even further into the future, then examined a particular sequence at greater length but, rather than starting a whole new sequence, he started halfway through a previous sequence that the computer had already produced. In other words, the new initial conditions for this equation were taken from the results of an earlier equation. The resulting sequence of Lorenz's new calculation should have been an exact duplication of the previous calculation, but instead Lorenz was surprised to see that the computer had produced wildly different results: "Lorenz saw his weather diverging so rapidly from the pattern of the last run that, within just a few months, all resemblance disappeared" (Gleick 16). Lorenz initially thought his 
computer must have broken, but it turned out that the divergence occurred because he had rounded off the number that the computer had produced and fed back into the initial conditions of the equation -thinking the difference would be inconsequential. This very slight difference between the two initial conditions resulted in completely different results: “[...] small errors proved catastrophic" (Gleick 17).

Lorenz conjectured from this experiment that his dynamical system, and therefore longterm weather, was unpredictable but he suspected that this unpredictability was more than the result of instability in the system (Gleick 22). Rather than trying to predict long-term weather patterns, Lorenz turned his attention to stability. He stripped back the equations he was using for his weather model until he was only left with three very simple nonlinear equations. These equations were so simple that they looked solvable, but they actually produced unpredictable results. For example, Lorenz's equations were used to model convection; the movement in fluid that is caused by hotter molecules rising to the top, and cooler molecules being pushed down to the bottom - as is evident in the way a pot of water behaves when it is reaching boiling point. If the temperature of the pot of water reaches boiling point, the movement of the fluid goes from being stable and predictable to unpredictable. The unpredictable behaviour of a pot of boiling water is the same as the behaviour of the Lorenz Waterwheel, and can be described or explained by the same differential equations. The effect of increasing the speed at which water flows into the waterwheel is the same as the effect that increasing the temperature of a pot of water has. The increased speed, and the increased temperature, also increases the nonlinearity of the system which leads to unpredictable behaviour that is determined by its previous states-it leads to chaos.

From here, Lorenz went on to illustrate the data produced from his equations in phase space: "Phase space gives a way of turning numbers into pictures, abstracting every bit of essential information from a system of moving parts, mechanical or fluid, and making a flexible road map to all its possibilities" (Gleick 134). In other words, phase space draws, or curves, how states are held together and apart in space for all initial conditions (i.e. all possible spins of the Lorenz Waterwheel that could occur). Lorenz's map provided a visual representation of the phase space curve within a dynamical system, and ultimately revealed 
what is known as the Lorenz attractor. The curve of the Lorenz attractor never intersected itself but would form loops and spirals that, after numerous iterations, revealed a shape or pattern: "[t]hose loops and spirals were infinitely deep, never quite joining, never intersecting. Yet they stayed inside a finite space, confined by a box" (Gleick 140). I will examine the Lorenz attractor in more depth in my discussion of strange attractors in Chapter Two but, broadly speaking, the Lorenz attractor revealed an underlying structure embedded in Lorenz's disorderly sequence of data (29).

Gleick explains that Lorenz's discoveries about instability in nonlinear dynamical systems were enthusiastically embraced. However, the ideas and discoveries behind chaos had actually already been explored in the 1900s by the mathematician Henri Poincaré, but had since been forgotten (Gleick 46). Poincaré's study of nonlinear dynamical systems arose out of his investigation into the instability that was exhibited in the three-body, or n-body, problem:

The two-body problem is easy. Newton solved it completely. Each body-the earth and the moon, for example-travels in a perfect eclipse around the system's joint center of gravity. Add just one more gravitational object, however, and everything changes. The three-body problem is hard, and worse than hard. As Poincaré discovered, it was most often impossible (Gleick 145).

In the same way that it becomes impossible to predict the behaviour of Lorenz's waterwheel when the flow of water reaches a certain speed, and the movement of molecules in a pot of water becomes impossible to predict when the temperature becomes hot enough, adding a third body into the two-body problem means that it is impossible to know what will happen to the solar system in the long-term. This unpredictability is due to the interlinked relationship between the movement and force of each body that is present in the system. Since the first body affects the second and third body, and the second body also affects the first and third body, and the third body affects the first and second body and so on, it is impossible to precisely capture how the system will behave. And so, just as Lorenz abandoned his attempt to predict long-term weather patterns sixty years later, Poincaré's solution to this three-body problem was to abandon any attempt to find an absolute or general solution; he acknowledged that it was impossible to know what such a system would do in every possible state or initial condition. 
Instead of asking what might happen next in the dynamical system of the three-body problem, Poincaré set out to describe the states of the system in phase space in order to get a better idea of these dynamics, making it easier to observe the system in all its possible states. Essentially, Poincaré discovered that if the phase space curve of the bodies remains within a bounded region the phase space curve would eventually, after a long but finite time, pass through a point that was very close to its initial state. The phase space curve never quite intersected but came very close to doing so. Poincaré concluded that these points of near-intersection were indications of simultaneous stability and instability within the system, and that the points formed a web so intricate as to be near-impossible to draw by hand (Gleick 142-145).

While Poincaré's study of these points of near-intersection was never investigated any further at the time, nearly a century later his study would be picked up by Stephen Smale, a topologist who developed what is known as the Smale Horseshoe map. Gleick describes the map:

A space is stretched in one direction, squeezed in another, and then folded. When the process is repeated, it produces a kind of structured mixing familiar to anyone who has rolled many-layered pastry dough. A pair of points that end up close together may have begun far apart (Gleick 51).

Smale's Horseshoe map (named because the process involves stretching the space into a horseshoe-shape) provided a visual analogy for the sensitive dependence on initial conditions that both Poincaré and Lorenz had discovered earlier: "[p]ick two nearby points in the original space, and you cannot guess where they will end up" (Gleick 52). Not only does the horseshoe map explain how the dynamics are stretched and folded away from one another in chaotic systems, its repeated iterations of folding and stretching leads to the formation of a fractal structure. A fractal is a recursive pattern that displays self-similarity across all scales and can be identified in the regularity that is embedded in irregular shapes in nature like a snowflake, clouds, or the coastline. The field of fractal geometry was led by Benoit Mandelbrot and, as Gleick explains, Mandelbrot's fractals provided a way to measure qualities that would otherwise have no clear definition (98). The presence of a fractal structure in Smale's horseshoe map, where certain points that start off far apart come 
together, provided an explanation as to how the phase space curve that Poincaré and Lorenz observed is able to come so close to intersecting without ever crossing itself. That is, the repeated points where the phase space curve appear to be intersecting were forming a web of self-similar iterations that went infinitely deep. The fractal structure of these points confirms the presence of regularity beneath the irregularity of the systems.

In essence, mathematicians who investigate chaos aren't interested in predicting what might happen in the future of unstable systems, but are instead interested in understanding the potential order and stability that lie beneath systems that display disorder and instability-the underlying behaviour of systems that are deterministic but unpredictable. My overall argument in this thesis is that the narratives of successful Seral dramas function in a way that is similar to how a nonlinear dynamical system that is deterministic but unpredictable behaves. As a result, the concepts of fractals and of sensitive dependence will be central in the comparisons that I make. However, in order to frame my argument in a more cohesive way, I will look at two areas within chaos that have fractal geometry and sensitive dependence embedded within them: "Universality" and strange attractors (which I will outline in more detail below).

\section{Methodology and Thesis Outline}

The accessibility of chaos through popular science texts-such as James Gleick's Chaos: Making a New Science, and Ian Stewart's Does God Play Dice?-has resulted in the emergence of chaos as a framework of investigation within the fields of social sciences and humanities. In Chaos Bound: Orderly Disorder in Contemporary Literature and Science and in her edited volume Chaos and Order: Complex Dynamics in Literature and Science, $\mathrm{N}$. Katherine Hayles - a rare humanities scholar with a background in both science and literary studies-provides an analysis of chaos as it relates to contemporary literature, and postmodernism in particular. Hayles uses chaos to read literature as having transformative potential through cognitive processing. She views the literary text as a complex dynamic, and uses chaos to understand how literature connects writer, reader, and text with the culture (30). A similar notion is also reflected in Patrick Brady's Chaos Theory, Control Theory, and Literary Theory or: A Story of Three Butterflies. Brady examines the problematic way in 
which we perceive reality as disordered but impose order upon it, and demonstrates how this process is reminiscent of the structures within structures posited by the structuralist anthropologist Claude Levi-Strauss and the developmental psychologist Jean Piaget (66). Jo Alyson Parker takes a slightly different approach and argues that reading narratives whose structures display chaotic qualities enables us to apprehend how their form is meaning, and how their meaning is dynamical due to the feedback loop between text and reader (2-3, 149).

There are also a handful of examples of chaos being used in other fields of the humanities. For instance, Laurence Bouquiaux examines the "increasingly complex world" through a philosophical lens that draws parallels between Leibniz's seventeenth century philosophy of monads and Mandelbrot's fractal geometry, with the conclusion that the universe cannot ever be fully known or understood. John Lewis Gaddis turns to chaos as a way to depart from traditional approaches to historical processes that tend to read history as a series of large events that connect through a clean line of cause and effect. In sociology, Hal Gregersen and Lee Sailer, and Russ Marion, argue that some social behaviour is hard to predict because the underlying social system is inherently chaotic, and this in turn means that social entities that may have virtually identical internal states, or exist in identical environments, can exhibit vastly different behaviours. In a psychoanalytical study, Robert M. Galatzer-Levy examines whether chaos can be used to support the idea that psychological actions are linked in a continuous way, but that these links cannot always be seen or sensed.

While there is little evidence of chaos being applied in media studies, one study by Harri Raisio and Niklas Lundström provides analyses of various films (Butterfly Effect, Mr Nobody and Chaos Theory) each of which incorporates chaos into its plots in different ways. Raisio and Lundstrom identify the various points of bifurcation within each of the film narrativeswhere the protagonists make decisions in the narrative that drastically change future outcomes-and they go on to evaluate the "successfulness" of the choices that they make. While their analyses are valid, the overall argument is undermined in that they are essentially applying chaos to real-world scenarios (identifying social systems as complex or dynamic systems) through the filmmakers' reductive application of chaos to their narratives. Similarly, in "From Fears of Entropy to Comfort in Chaos: Arcadia, The Waste Land, 
Numb3rs, and Man's Relationship with Science", Kirsten Miller examines the way in which particular examples of fiction have incorporated chaos into their themes and storylinesespecially those that explore free will and the human experience. While she provides in depth analyses of particular texts, Miller-like Raisio and Lundström-tends to inaccurately use chaos to mean disorder, pure randomness, or a lack of control.

Indeed, many scholars have been criticised for their similarly generalised and metaphorical applications of chaos. Adrian MacKenzie, for instance, postulates that it is common for academics in humanities to locate chaos at the simple end of the complexity spectrum (46). MacKenzie recognises that the metaphorical borrowing of complexity can result in the mobilisation of concepts and ideas, but he claims that this process will not necessarily lead to the transformation or invention of concepts (59). He goes on to argue that complexity theory, and chaos, should not be used to find solutions in other fields, but can be useful to problematise what may be perceived as simple by introducing uncertainty.

In The Ends of Metaphor: Literary Analysis and Chaos Theory, Merja Polvinen examines what she believes can and cannot be achieved by combining chaos and literary analysis. Polvinen suggests that a large number of scholars, such as Susanne Vees-Gulani and Gordon E. Slethaug, who are interested in using chaos in literary criticism, do so because they wish to analyse works in which chaos appears as a thematic or structural element. In these instances, the tools for literary analysis are not reliant on a deep knowledge of chaos but remain within the discipline.

From these texts we can see that different methods have emerged in terms of how chaos can be applied to subjects within the humanities and social sciences. At one end of the spectrum are studies that use chaos in a reductive and purely metaphoric way, and as a result fail to bring a new perspective to their topic. At the other end of the spectrum are studies that make the impossible argument that the "texts" that are being studied are chaotic systems. For the purpose of this thesis, I will be employing a method that sits somewhere between these two approaches. That is, I am fully aware that my background in the arts limits my ability to understand and apply the mathematics and physics that underpin chaos, and I am aware that this will inevitably lead to a fairly generalised and 
metaphorical understanding and application of chaos, rather than a practical one. My justification for this is that I am not setting out to argue that serial drama narratives are chaotic systems, but I am using chaos as a comparative tool to examine, and bring light to, the fundamental building blocks that exist in all serial dramas, and how they are used in unique, innovative and complex ways.

In saying that, my aim is to be as detailed and non-metaphorical as possible. One way in which I set out to do this is by examining narrative structures through the lens of chaos, rather than simply analysing the content of serial dramas; I am looking at how stories in serial dramas are told in a chaos-like way, and not simply how stories in serial dramas are about chaos, or employ themes of chaos. It is also important to note that I am approaching this thesis from the perspective of a writer, rather than a scholar, of serial drama narratives. That is, rather than analysing the complexity of serial dramas in terms of factors like authorship, genre, stylistic tendencies, industrial practices and the incorporation of transmedia, I will be examining the narrative structures of serial dramas at a molecular level-how the narratives of serial dramas are conceived and written, as well as analysing serial dramas as finished products. This is not to say that I don't believe the broader factors mentioned above aren't significant or relevant to the nature of complexity in serial dramas, it is simply that my perspective of "complexity" is slightly different; rather than focusing on the differences of serial dramas, I will be looking at the techniques and structures that remain the same. Because I am approaching this thesis from the perspective of a writer, chaos will be the primary framework with which I will be examining the narratives of serial dramas, and the scholarship around TV drama narratives will be used as a secondary framework to support and guide my discussion and analyses.

As mentioned earlier, I will be framing my analysis of serial drama narratives around two aspects of chaos-"Universality" and strange attractors. My first chapter will use Mitchell Feigenbaum's theory of "Universality" as a framework in order to re-examine the rules and formulae that underpin successful serial drama narratives. "Universality" is the discovery that dynamical systems that are transitioning from stable to chaotic follow a simple rule (known as the Feigenbaum constant) that remains fixed within the system despite how disorderly such systems may appear on the surface. Feigenbaum argued that this rule not 
only remains fixed within a particular system, but the very same rule can be found in all dynamical systems on their way to chaos. Feigenbaum's ideas correspond to, and incorporate, Mandelbrot's fractal geometry and his observation that variation across scale was not arbitrary, but rather followed rules (162). I will use "Universality" and Feigenbaum's constant as a way to frame my examination of how narrative structures and patterns are repeated within a serial drama, as well as across different serial dramas. I will argue that on a structural level all serial dramas use the same fundamental building blocks, and I will examine how these building blocks are being used to produce original and complex stories. I will largely be using "how to write TV" books as a way to outline the rules and formulae, and will provide an analysis of The Wire (HBO, 2002-2008) in light of my findings.

In my second chapter I will build on my discussion about the repeated structures and rules that underpin TV drama narratives to argue that a successful serial drama must find a balance between being predictable and unpredictable. I will examine the unpredictability of serial drama narratives through the lens of strange attractors which, when depicted in phase space, display a curve that never quite intersects itself but repeatedly comes so close to doing so that it, after numerous iterations, forms a distinctive shape or pattern. Gleick's description of a trajectory that is being pulled towards a Lorenz attractor, for instance, could also be used to describe how I perceive the narrative of a TV serial as a whole: "It always stayed within certain bounds, never running off the page but never repeating itself, either [...]" (30). A strange attractor demonstrates how a system can be deterministic yet unpredictable, similar to how a serial drama must be expected but also unpredictable. I will be providing an analysis of The Leftovers (HBO, 2014-2017) to demonstrate how serial dramas display similar characteristics to the continuous curve of a strange attractor.

My creative component represents a more practical application of chaos and serial drama narratives. Rather than only using chaos to analyse serial dramas that have already been created, I will be applying my critical findings to the creation of my own serial dramas. Above all, my intention with my creative component is to write three TV serials that are engaging and entertaining, not to write three TV serials that purely resemble chaotic systems. In other words, I am not using chaos as a way to transform the fundamental narrative structures of a TV serial, but am rather using chaos as a way to re-examine the 
inherent structures and patterns of TV drama narratives as a kind of exercise or challengeas a beginning writer. I will use this exercise to familiarise myself with the inherent structures and patterns whilst simultaneously employing these structures and patterns in an innovative way.

My first TV project-Untold-is a fantasy serial that is largely set in the psychopathic mind of Letha Maven, an elderly woman who has been sent to prison for suffocating her own babies 50 years earlier. Letha Maven tells a series of embedded stories to her fellow inmates as a way to entertain and manipulate them. Letha, however, doesn't realise that the main character in her story-Sara-has come to life in her head, and is navigating her way through Letha's memories, traumas, dreams, and stories in an attempt to gain free will. I have tried to use the fragmented nature of TV drama narratives in this serial to incorporate embedded and self-similar narratives within it (in the mode of 1001 Nights), and as a result the serial has fractal geometry at its core.

My second TV project-Bad News-is about a group of misfits who set up a business that provides the service of breaking bad news for, and to, other people. Each episode is centred around a case, and is mixed in with the ongoing trials and tribulations of the members of the Bad News business. Bad News was written through the lens of strange attractors in that each of the cases are interlinked without the characters realising it; one person's bad news leads to another person's bad news and so on. In this sense, the characters are inadvertently creating their own "phase space curve" that never intersects but repeatedly comes very close to doing so, and as a result their narrative trajectories are continuous yet episodic.

My third TV project-The Chances - tells the story of seven people throughout history who are all named Bertha, who all look alike, and who all learn about the Berthas who have gone before them at different ages and in different eras. I have written this project through the lens of sensitive dependence to initial conditions in that the varying reactions the Berthas have to finding out about each other determine the subsequent courses of their lives, and also affect how the other Berthas are forced to live their lives. My intention with this serial is to consciously utilise the way in which serial drama narratives generate variety through repetition. And it is in this repetition that I want to establish the links and connections 
between each of the Berthas - in a way that allows me to depict their individual stories, but also combine their stories to form a narrative whole that resembles a nonlinear dynamical system.

While each TV project is written with a particular characteristic of chaos in mind, these characteristics inevitably overlap-by focusing on fractal structures in Untold, for instance, I am also employing the ideas of strange attractors and sensitive dependence. One characteristic of chaos doesn't function independently of another when considering a dynamical system as a whole, just as one element of a serial drama narrative doesn't function independently of another when considering a serial as a whole. In this sense, the unpredictable motion of the Lorenz Waterwheel is an apt analogy for how the interconnected components of serial drama narratives function to produce storylines that are always the same but always different. This analogy will become clearer by the end of this thesis. 



\section{CHAPTER ONE "Universality" and the Narrative Structures of TV Dramas}

\section{Introduction}

In their instructional book, Successful Television Writing, Lee Goldberg and William Rabkin write, "Every TV drama series is the same [...] It's true, there are great differences among these shows. But they're differences of ambition, of execution, of style. In terms of structure, of design, of production [...] they are fundamentally more similar than different" (13). A similar notion is found in most "how to write TV" books, and is a clear indication that, underneath all their differences, all serial drama narratives are actually made up of the same, simple structures. In this chapter I will be using the lens of "Universality" to examine how serial dramas utilise the same fundamental building blocks in innovative and complex ways.

"Universality" emerged in the 1970s, and is largely attributed to the mathematical physicist Mitchell Feigenbaum. The reason "Universality" is a useful lens through which to unpack the fundamentals of serial drama narratives is that it is, in very basic terms, the discovery that unpredictable systems can be understood through a single, simple equation. According to James Gleick, "[u]niversality offered the hope that by solving an easy problem, physicists could solve much harder problems. The answers would be the same" (180). Instead of trying to understand unpredictability through a complex lens, Feigenbaum focused on the simplest equations he could find as a way to comprehend unpredictable behaviour. In a similar way, this chapter will examine the complexity of successful serial drama narratives by focusing on the simple structures, formulae and rules that underpin them. I will begin by giving a brief introduction to "Universality" before moving on to a comparison of "Universality" with the specific narrative structures and rules that, I argue, underpin all serial dramas. The explanation of these structures will largely be drawn from "how to write TV" books, and will focus on act structure, episodic structure, season arcs and series-long arcs, as well as the execution, ambition, and style that Goldberg and Rabkin mention. I will finish the chapter by providing an analysis of The Wire, a show lauded for its complex and ground-breaking storytelling but that still, I argue, adheres to the same fundamental narrative structures and rules as any other serial drama. 


\section{"Universality"}

James Gleick describes how in 1974 Mitchell Feigenbaum knew that the idea of hidden unity and a common underlying form in nature was starting to become a cliché, and that to make something substantial of the idea physicists needed a practical framework that would turn these ideas into calculations. Feigenbaum developed his own thinking from ideas that had already been forming amongst a number of physicists, such as Kenneth Wilson, Leo Kadanoff and Michael Fisher who suggested that seemingly unrelated phase transitions followed the same rules (161). Their findings showed that the smooth and predictable behaviour that such matter would demonstrate when changing from one state to another was little help in understanding the actual transitions themselves. A pot of water on the stove, for example, will demonstrate regular and predictable behaviour as it heats up, until it reaches boiling point and something interesting happens at the molecular interface between liquid and gas - the change in temperature pauses (Gleick 160). In a similar way, a block of metal being magnetized will also undergo regular behaviour until it reaches a particular point at which it must make a choice as to which way it will be oriented. Kadanoff explained this process in terms of scaling - that each smaller piece of metal that makes up the whole piece of metal must be communicating information to each other to finally come to the same decision (Gleick 162). Melanie Mitchell explains this phenomenon when she argues that single entities are limited in their potential activity, but when these single entities come together they produce complicated and sophisticated systems as is seen in the brain, in ant colonies, immune systems, and in economics (6). What Kadanoff observed, however, was that these single entities not only came together to form a more complicated whole, the single entities were also iterations of the whole.

This idea of iterations, or scaling, corresponds to Benoit Mandelbrot's observations in fractal geometry, that variation across scale is not arbitrary, but rather follows rules. As Gleick puts it:

Variability in the standard measures of mass or length meant that a different sort of quantity was remaining fixed. In the case of fractals, it was the fractional dimension-a constant that could be calculated and used as a tool for further calculations. Allowing mass to vary depending on scale meant that mathematicians could recognise similarity across scales (162). 
Kenneth Wilson took Kadanoff's scaling process a step further and converged these ideas with the "Renormalisation Group Theory", which was used when facing the messy problem of calculating items of infinite quantities. Renormalisation got rid of the infinities, creating a shortcut by acting as if a quantity such as mass was not fixed but could fluctuate depending on the scale from which it was being observed (161). Feigenbaum latched onto Renormalisation, finding it useful to identify "ranges" within a particular system as a way to reduce the amount of information or noise needing to be observed in order to form a conclusion. His thinking was, if you can apply the findings taken from a small pool of information to the larger pool via scaling, then you can solve complex problems in a much easier and more efficient way (Feigenbaum 16).

But, as Gleick describes, this process was by no means perfect, as renormalisation still did not explain the "mysterious moment when an orderly system turns chaotic" so Feigenbaum eventually turned to Robert May's study of population biology as a way to come to a better understanding of this "mysterious moment". He examined the following basic difference equation:

$$
y=r\left(x-x^{2}\right)
$$

In population biology, $x$ is used to represent the current population of a specific species, and $y$ represents the future population. So, when presented on a curve, if $x$ is small, then $y$ is going to be small, but larger than $x$ (the curve rises on the graph). If $x$ is in the mid-range, then $y$ will be large. But at a certain point-when $x$ is large- $y$ tapers off and becomes small again, thus representing the way in which a population might crash when it grows too large for the maximum carrying capacity of the environment (Gleick 166). Since Feigenbaum was interested in understanding the behaviour of a system en route to chaos, he used this simple equation repeatedly, creating a feedback loop, to see what would happen to the sequence of values - whether the repeated iteration would create periodicity. He fed the output of one calculation into the input for the next equation; the value of $y$ in one calculation would become the value of $x$ in the next calculation and so on. What he found was that the sequence "bounces from place to place on the parabola at first, and then, perhaps, homes in on a stable equilibrium, where $x$ and $y$ are equal and the value thus does 
not change" (167). Essentially, this process proved that a simple equation repeated over and over again produces unpredictable, rather than periodic, results.

Unbeknownst to Feigenbaum, Edward Lorenz had been dealing with a similar issue in 1964 in his investigation of whether a climate actually exists. He wondered whether periodic behaviour would occur, in terms of climate, when creating a feedback loop:

[...] Lorenz first examined what happened as the equation was iterated, given some parameter. With low parameters he saw the equation reaching a stable fixed point. There, certainly the system produced a "climate" in the most trivial sense possible - the "weather" never changed. With higher parameters he saw the possibility of oscillation between two points, and there, too, the system converged to a simple average. But beyond a certain point, Lorenz saw that chaos ensues (Gleick 168).

Feigenbaum later went on to investigate how and why chaos occurred beyond this certain point, and discovered that when the parameter values of a system en route to chaos reach a certain point, bifurcations, or period-doublings, occur (Boeing 5). That is, the system will shift in behaviour by doubling in value; the population of a species, for instance, might change from a four-year cycle, into an eight-year cycle, exponentially increasing the rate of population (Gleick 171; See figure 1). It was this occurrence of bifurcation that Feigenbaum was especially interested in. In his own words:

[...] for some range of parameter values, the system exhibits orderly periodic behavior; that is, the system's behavior reproduces itself every period of time $T$. Beyond this range, the behavior fails to reproduce itself after $T$ seconds; it almost does so, but in fact it requires two intervals of " $T$ " to repeat itself (Feigenbaum 17).

In other words, the period doubles so that it becomes $2 T$, and it remains that way across a certain range of parameter values before another parameter value is reached which almost causes the behaviour to reproduce itself again, but it cannot do so without becoming 4T. This "process of successive period doubling recurs continually [...] until, at a certain value of the parameter, it has doubled ad infinitum, so that the behavior is no longer periodic" (Feigenbaum 17). 


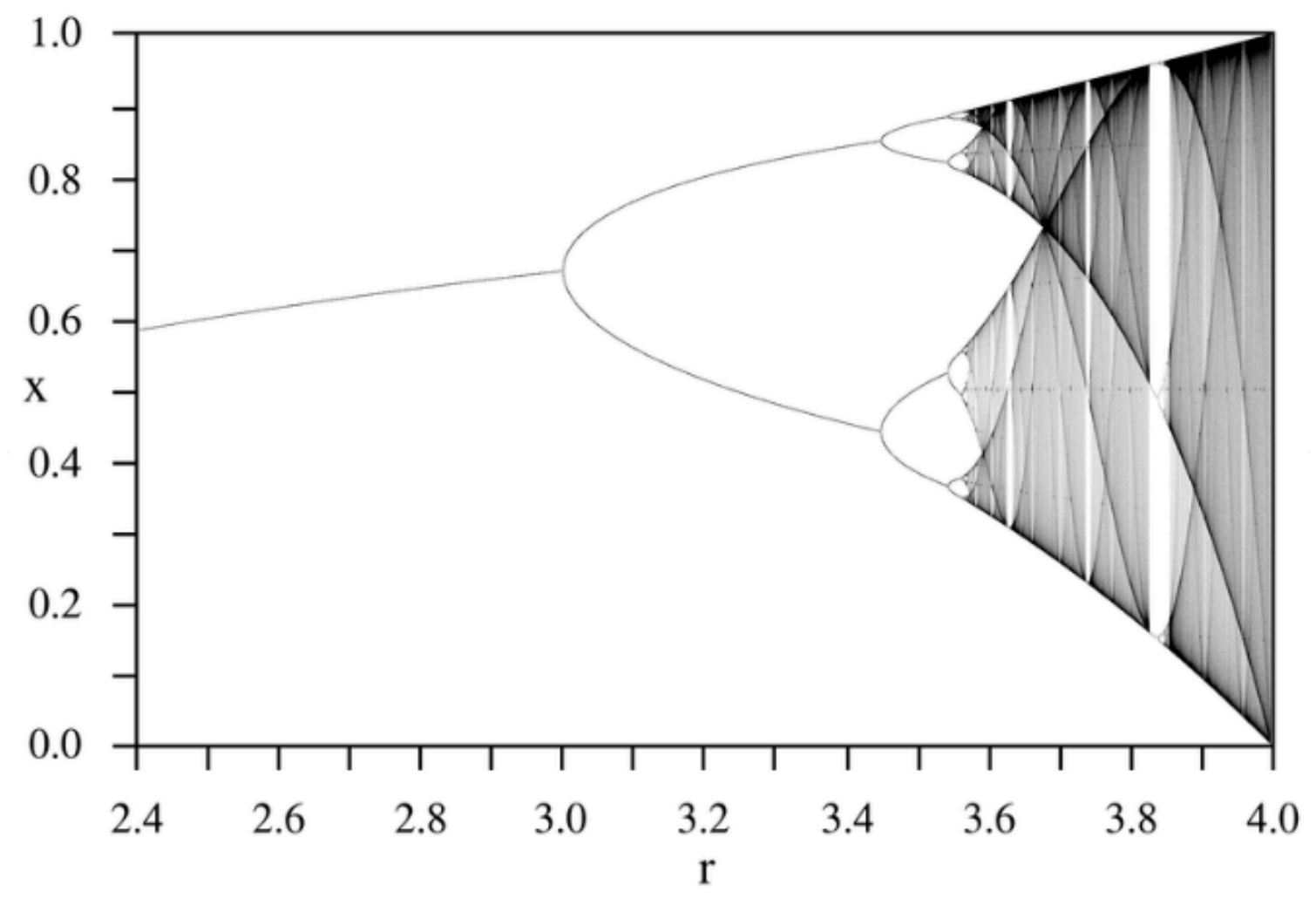

Figure 1: The LOGISTIC MAP demonstrates the increasing rate of bifurcation as a system approaches chaos, image retrieved from: Wikimedia Commons, (PAR, 14 Sept. 2005), <upload.wikimedia.org/wikipedia/commons/7/7d/LogisticMap_BifurcationDiagram.png>

We can see in the figure above that, as a population grows and moves from order to disorder, the points of bifurcation come faster and faster. Feigenbaum calculated the exact parameter values that would lead to a split or doubling, and found an unexpected regularity in his calculations that showed the numbers were converging geometrically the way "a line of telephone poles converges toward the horizon in a perspective drawing"; if you know the ratio between the length of the first two poles then you know the rest as well due to their self-similar scaling properties (Gleick 172). In this sense the period doublings were not just coming faster and faster, but they were coming faster at a constant rate (172). Feigenbaum found that there was a consistent pattern in systems that are heading towards chaotic behaviour. This is reflected on the logistic map diagram, where the bifurcation points occur so close together that they appear incomprehensible and convoluted; in reality there is precise regularity underneath this image of unpredictability. 
Not only did Feigenbaum discover the constant rate at which the bifurcations occurred, he also calculated the ratio of convergence to the most precise number possible and thus discovered what is now referred to as the Feigenbaum Constant (Goldstein 2; Gleick 172):

\subsection{9}

This constant ultimately means that the points of bifurcation aren't necessarily happening randomly, or even by an external variable being changed-it's happening because some kind of deterministic relationship between the parameters exists (Mitchell 33).

Feigenbaum's constant proved that some regularity lay beneath the turbulent surface of his equation and, to add to this level of regularity, he later found the identical geometric convergence occurring in a different, and much simpler, trigonometric function, which he in turn used, to catalogue topological patterns. Again, he calculated the ratio of convergence to the most precise number and got the same result as before: 4.669 (Gleick 172): "[n]o mathematical or physical theory existed to explain why two equations so different in form and meaning should lead to the same result" (173). He then tried several other functions that went through a sequence of bifurcations on the way to disorder and every one of them eventually produced exactly the same number: "[...] something in the heart of these very different equations, repeating over and over again, created a single number" (173). The physical application of Feigenbaum's theory of "Universality" suggested that real-world systems (e.g. boiling water, population growth, the noisiness of a large variety of mechanical, electrical, and chemical oscillators) possess a mathematical characteristic that is the same and would therefore be measurably the same (Gleick 173; Feigenbaum 17).

While I am very aware that a serial drama is not a dynamical system that is transitioning from order to chaos-and that Feigenbaum's constant of 4.669 is not hidden within the narrative structures of various serial dramas -1 do believe that the principles of "Universality" will be useful in unpacking the simple structures and rules that underlie all critically successful serial dramas. I will use "Universality" to examine how these structures and rules are not only useful for creating complex and innovative stories in serial dramas but are actually necessary for creating complex and innovative stories. In other words, there 
are several "constants" within serial drama narratives that, if abandoned or lost, would result in a lack of cohesion and regularity that would then likely result in a loss of viewers. Just as Feigenbaum's discoveries surrounding "Universality" were useful for solving complex problems in a simple and efficient way, I will use "Universality" to demonstrate the way in which all serial dramas are comprised of the same basic narrative structures.

In order to argue this point, I will compare the fractal nature of a system on its way to chaos to the "self-similar" units that constitute a serial drama narrative-a serial is made up of seasons which are made up of episodes which are made up of acts which are made up of scenes. Each of these narrative "units" must reflect each other at different scales yet must also work together to produce a narrative that progresses and advances in an engaging way. I will then go on to compare Feigenbaum's constant to the way in which any successful serial drama has a self-imposed tone, style, or "execution" that is set up at the start of its run and that must remain consistent throughout the entire serial yet must also be used in a way that creates new and fresh stories.

\section{"Universality" and the Narrative Structures of TV Drama}

In her book How to Write for Television, Madeline DiMaggio writes: "When you read television scripts [...] you will be amazed at how simple they appear. The simplicity is an illusion because a good script is so economical" (9). In other words, TV writers must only include that which is vital for telling the story at hand which means that despite the tendency of serial dramas to relate complex, sprawling narratives, they are constructed of much simpler components than they might appear. In order to be economical in this way, several rules and constraints have arisen within the narrative form, but, rather than limiting or stifling creativity, these restrictions and rules can be used in ways that stimulate creativity and variety. In Jason Mittell's words, "[s]uch constraints work to limit how television stories can be told but also provide clear structures within which innovations can flourish, creatively challenging well-established norms" (33).

As well as utilising these structures, a successful serial drama must also make sure that these structures remain hidden from an audience's view: "[o]nce you have the rigid frame of the 
puzzle, you have to hide the puzzle so the audience isn't aware they are being manipulated" (Rabkin and Goldberg 138). And so, just as Feigenbaum unveiled the intricate order and repetition that is underlying the complexity of various systems en route to chaos, it's only by looking at a successful serial drama narrative very closely that we can detect the same patterns being repeated over and over again. As is outlined in most 'hto write TV' books, every episode within a series or serial is broken down into four acts. Goldberg and Rabkin claim that most people will know the four-act structure intrinsically: "The reason you know it is because you've been conditioned by the predictable rhythm of cinematic moments in the story, and the interruption of commercial breaks at the end of each act" (19). My condensed version of Goldberg and Rabkin's break-down of the episodic act structure goes as follows:

Act One:

We are introduced to the characters, the conflict, and what is at stake.

\section{Act Two:}

The hero/ heroes embark on their course of action to resolve the conflict, but new obstacles are thrown in their path-thrusting the story in a new and unexpected direction.

\section{Act Three:}

The hero reacts to the change in the situation and embarks on a new course of action that leads them to believing all is well, only to find out they are wrong and things are much worse. There appears to be no hope.

\section{Act Four:}

The hero comes up with a solution, overcoming their obstacle, resolving the conflict and achieving their goal (19-21).

Goldberg and Rabkin add that some shows "have teasers and tags and also divide their four acts in half to cram in more commercials, but despite these permutations, they all rigidly conform to the same basic structure" (21).

As Epstein explains, each act is approximately ten minutes or so, and-for network or broadcast shows-after each act we generally cut to a commercial which stops the flow of the narrative dead (93). In order to maintain the audience's attention between commercial 
breaks, each act ends with what Epstein calls "Act Outs": "The last thing that happens before a commercial-an event that is so compelling that an audience has to come back to find out what happens next" (100). Epstein emphasises the fact that an "Act Out" doesn't necessarily have to be a dramatic cliff-hanger every time, but must answer the question that is raised at the beginning of the act, and in turn raise new questions that drive the narrative forward (100).

Of course, with TV drama's fairly recent shift into what Dunleavy calls a "multiplatform" era, these industrial constraints no longer have as much impact on how a serial drama is told; narrative strategies like "act outs" and easy-to-follow narrative linearity are no longer a requirement of the narrative form: “..multiplatform television has revealed its capacity to work in radically different ways from the economic models that shaped American television's development as 'broadcast television' and then 'broadcast and cable', paradigms that privileged and/or enabled certain forms of TV drama" (Dunleavy 11). As such, the above outline of the episodic structure and act structure might seem like it is describing the structure of a series or procedural that is made for broadcast TV (in which storylines will often revolve around a "problematic", are generally developed and resolved within single episodes, and must work around ad breaks), rather than a non-broadcast serial (in which episodes are interrelated, interdependent and continuous, and do not need to work around ad breaks) (Dunleavy 101-102). However, as I will outline below, serials still adhere to this general episodic and act structure, but they do so in a slightly less rigid and literal way. That is, where a series will often begin by literally introducing a new character or set of characters (such as a patient in a hospital, a victim of a crime, or a criminal) who will only feature in that episode, a serial will usually set up the main characters, who are already well-established within the show, in a situation or circumstance that will be explored in that specific episode. This situation or circumstance will usually present some kind of conflict that the character or characters will set out to overcome in the second act, only to fail or be thrown off course in some way. In the third act, the character or characters will find a new way to approach the conflict, only for things to get even worse. In act four of a serial, the conflict that was set up at the start of the episode will be resolved in some way. This resolution might be less closed-off in a serial than in a series, but this does not mean that each episode of a serial does not come to some sort of resolution. A serial will provide a 
resolution, but it will do so in a way that presents new questions to be explored in the next episode.

Breaking Bad (AMC, 2008-2013) is a clear example of how a show that is considered a complex serial drama continues to implement this episodic and act structure. For instance, this is evident in episode 7 of season 3 of Breaking Bad, titled "One Minute". This episode opens with a teaser that provides a flashback scene of the "Two Cousins" as children being taught a harrowing lesson by their Uncle about the importance of family, before flashing forward to the present day where "The Cousins", now adults, are plotting to go after DEA agent, Hank, for killing their cousin, Tuco. Here we can see that the main conflict for this episode is going to be between "The Cousins" and Hank. The stakes of this conflict are especially high, now that we've seen how the importance of family has been drilled into "The Cousins" since they were kids. This conflict is further cemented in act one in which Hank jeopardises his career after he beats Jesse to a pulp for tricking him into thinking his wife was in an accident in the previous episode. In this first act we also see Walt who is rattled when Jesse threatens to go after Hank, and also sabotage Walt. Hank's conflict and stakes for this episode are that he might lose his job, which, to him, is his whole identity. Walt's conflict and stakes for this episode are that he might have to choose between killing Jesse or going to jail, unless he can think of another way to stop Jesse from acting out. So, while we are not being introduced to a new character or set of characters in the first act, we are "re-introduced" to characters we are already familiar with in a way that sets up what their episode-specific conflict is (even if this conflict is stemming from previous episodes).

In act two of this episode we see that Hank's course of action to overcome his obstacle is that he pleads the fifth in his statement to the DEA. His obstacle is that Jesse is pressing charges, and his new direction is that he breaks down and shows his vulnerability for the first time. Meanwhile, Walt's act-two action is that he is avoiding Jesse (hoping that Jesse doesn't act out). His obstacle is that Skyler asks him to talk Jesse out of pressing charges against Hank (and this calls back to the overarching theme of family loyalty, that was set up in the teaser). The Cousins' actions are also raising the stakes of the episode's main external conflict between The Cousins and Hank - even though Hank is not aware of this. 
In act three of this episode Hank's new course of action is his intention to tell the truth at his hearing. And his moment of no hope is in his admitting to his wife that his days as a cop are over-not because of Jesse, but because he's been unravelling mentally and physically for a while. Meanwhile, Walt's new course of action in this third act is getting Jesse a job in his lab so that he won't press charges or sabotage Walt. And his moment of no hope is when Jesse lashes out, and throws his job offer back in his face. Both Hank and Walt are at their lowest point at this point in the episode.

In act four of this episode we see that Hank's solution is that he successfully defends himself against "The Cousins" - without his gun - when they finally come after him, thus redeeming himself (and temporarily overcoming his loss of identity). Walt's solution is that Jesse agrees to his job offer after Walt finally pays him a compliment-he is going to be Walt's partner, meaning that Walt doesn't have to kill him, and Jesse can no longer sabotage him. The main conflict between Hank and The Cousins that was set up in the teaser is resolved in the final act in a way that gives the episode its own distinct shape from the other episodes-but this doesn't mean that the storylines in this episode are over, as they might be in a series.

And so, while the ends of each act in a serial aren't necessarily built around commercial breaks, as they are in traditional broadcast TV narratives, it is evident that this act structure persists in all serial dramas to some degree. As Epstein notes, "[...] experienced writers generally write with act outs somewhere in the back of their minds because they're used to it" (103). The persistence of "act outs" is not only due to this structure's long, embedded history in TV drama, but because the "act out" structure facilitates rises and falls in the narrative, which ultimately contribute to the forward drive of an episode, and in turn the entire serial; the ad-break act structure forces continuous change in circumstance and therefore keeps a story moving. As Michael Newman writes, the persistence of the four-act structure serves a variety of interests:

From an aesthetic perspective a four-act structure achieves a sense of proportion and symmetry, ensures a steadily rising action, and organises patterns of attention and expectation, with first acts opening causal chains that are carried across the second and third acts to be resolved[...]in the fourth (21). 
Madeline DiMaggio discusses this forward movement of fictional TV narratives by breaking down the elements of TV drama into "units". She points out that beyond the 4-6 acts that make up an episode, there are other types of "units" at various scales. DiMaggio considers the "scene" as the fundamental building block of a TV script that moves the story forward: "Imagine a set of building blocks. As the set of building blocks come together, they form a complete creation" (26). Sandler makes a similar point that, like the story as a whole, every scene needs to have a narrative structure of its own-a set up at the beginning, a power switch in the middle, and a resolution that then drives you into the next scene (118). These individual scenes work together in building an act, and the acts work together in building the episode (and from there, each episode works together in building the season, and each season works together to form the serial-narrative as a whole). As DiMaggio points out, each of these "units" can be seen as having the same purpose of driving the story forward, in a way that is not dissimilar to the scaling properties that Feigenbaum emphasises are integral to our understanding of non-linear dynamical systems. A dynamical system that is en route to chaos is made up of smaller single entities that not only work together and communicate to drive the whole to greater intricacy, but are actual iterations of the whole; by looking at a smaller range of information, we can understand a much larger range of information. DiMaggio posits a pseudo-equation for each unit at all scales, that we could characterise as "self-similar". For DiMaggio, every scene, act, episode and season of a TV series or serial can be reduced to a combination of the following components:

\section{Character + Dramatic Need + Obstacles + Conflict = Action (107).}

While hardly the same as the difference equation that Feigenbaum used to uncover the regularity of systems en route to chaos, the above does serve a similar purpose in representing the various components of each "unit" in a serial drama. The "equation" demonstrates how a serial's narrative units work together in driving the story towards future events, and thereby essentially forming the entire scope of a serial drama. Every unit of a serial adheres to the same general structure-a character who wants something faces obstacles that drive them to do particular things in order to get the thing that they want. In other words, a character's objective in a particular scene, should emulate and also contribute to the larger objective a character has in an act, and the character's objective in 
an act should, in turn, emulate the character's objective over the course of the entire episode, season, and serial. While this model is not specific to serial dramas, the fact that serials use an open, ongoing narrative form-as opposed to the self-contained and enclosed narratives of a film or novel, for instance-means that, as a whole, a serial drama may appear to go in erratic and unpredictable directions when in actuality the same thing is happening over and over again, just to varying degrees and at different scales. In Gleick's words, "[s]omething about these functions, must be recursive, [Feigenbaum] realized, selfreferential, the behaviour of one guided by the behaviour of another hidden inside it" (179).

Again, Breaking Bad serves as a clear example of this self-similarity within serial dramas. in which the main character, Walter White, arguably repeats the same set of actions across the entire serial, but increasing the rate of repetition at an exponential rate. Walter White starts off manufacturing and selling drugs in order to pay for his cancer treatment, but as he becomes further and further embroiled in the drug scene he loses sight of his initial motivations and instead manufactures and sells drugs simply for the money, and ultimately power and reputation. As Walt artfully navigates the obstacles and challenges that come his way, he grows stronger and stronger within the drug community; because he grows stronger, his actions become more extreme, and because his actions become more extreme the consequences of his actions are more extreme still-and so on, until Walt's actions and consequences converge, ultimately reaching their limit and marking the end of the serial. Within this trajectory, Walt's basic pattern of behaviour doesn't really change, it simply expands.

Obviously, the self-similar units within a successful serial drama are not only used to produce linear stories about a single character and their individual journey. Breaking Bad, for instance, does not only tell the story of Walter White; it's also a story about Jesse Pinkman, Skyler, Walt Jr., Hank, Saul Goodman, to name a few. As Ellen Sandler points out, every episode of any TV show will have an 'A' story-the main storyline that follows the main character-as well as ' $B$ ' and ' $C$ ' storylines: "[...] smaller, secondary stories that involve the supporting characters' problems that can either be related to the ' $\mathrm{A}$ ' story, or completely separate. The more ensemble the show, the more important the ' $B$ ' and ' $C$ ' stories are" (82). Mittell posits that the way complex TV dramas interweave A and B 
storylines can be considered a "narrative special effect" -TV's equivalent of the visual special effects of cinema. That is, viewers relish in observing how the creators of a serial will have its various story threads clash and combine in interesting ways (43).

As well as providing a break from the main storyline, these separate ' $\mathrm{B}$ ' and ' $\mathrm{C}$ ' stories also work to unify all of the storylines through self-similarity. Epstein describes ' $A$ ', ' $B$ ' and ' $C$ ' stories in a similar way to Sandler, but argues against the inclusion of "completely separate" storylines, for risk of the narrative becoming too confusing:

[...] in the most sophisticated shows, especially in complicated serial dramas such as The Sopranos, several stories can be going on in the same scene. Just as in real life, a character who wants one thing bumps into a character who has an entirely different agenda. Naturally, their agendas conflict [...] Nevertheless, keep your stories clear. In any given story, for any given character, one dramatic thing is going on at a time (120).

Epstein provides the term, "rule of one", to demonstrate that no matter how convoluted or confusing the storylines in a TV drama might appear, there is always something that remains constant within them: an aspect in each strand that unifies them. Gerald Kasey makes a similar case to Epstein's "rule of one" but discusses it in relation to a TV show's theme. He states that there isn't a limitation to what can be used in sub-plots, but it "mustn't be allowed to overshadow the basic story, and to be truly relevant it must ultimately flow into the main theme and become a vital part of it, like a stream joining and losing its own identity in a river" (96). With Kasey's river analogy in mind, we can see that the relationship between the ' $A$ ', ' $B$ ' and ' $C$ ' storylines in a TV serial is similar to Feigenbaum's examination of bifurcation points; when a slight change in a system causes it to shift in behaviour by exponentially doubling in value over time, in turn, giving the appearance of irregular behaviour that is, in actuality, an iteration of what came before, and what is to come. Just as each unit in a serial drama must, on some level, iterate the whole, ' $\mathrm{B}$ ' and ' $\mathrm{C}$ ' storylines can diverge from the main storyline but must, in the end, have a connection or resemblance to it; the ' $A$ ', ' $B$ ' and ' $C$ ' storylines must converge in the same way that Feigenbaum's bifurcation points converge geometrically (Gleick 172). 
For example, the ' $\mathrm{B}$ ' and ' $\mathrm{C}$ ' storylines in Breaking Bad that follow the supporting characters provide breaks in Walt's main storyline, while simultaneously serving his storyline. In the final episode of season one, for instance, Walt's wife, Skyler, goes to a jewellery store to return an expensive baby tiara that her sister, Marie, has gifted her, only to find that Marie had stolen it. When Skyler confronts her about it, Marie refuses to take responsibility. Later, Skyler talks disparagingly to Walt about her sister's antics, and considers dobbing her sister into the police. Walt is disturbed to hear his wife's low tolerance for immoral behaviour. At first, Skyler's storyline appears unrelated to Walt's, until this moment when Walt realises he can't tell Skyler the truth about what he has been up to, for fear of her dobbing him in. While initially seemingly insignificant and tangential to the main storyline, Skyler's storyline eventually merges with Walt's and, in fact, determines the direction of the following major storylines to come, by eliminating the immediate possibility of Walt coming clean to his wife. This example is one of many, and shows us that the supporting characters in Breaking Bad-and all successful serial dramas-are often used as obstacles in, or vehicles for, the main storyline. $B$ and $C$ storylines are inevitably going to be about how the events in the main storyline are impacting the minor characters in the minor storylines, or vice versa. What this ultimately shows us is that, despite their potential to be completely erratic, sprawling, and confusing, no storyline in a successful serial drama is ever random or extraneous. This is similar to Kadanoff's explanation of the scaling process that occurs when a metal becomes magnetised-single entities not only come together to form a more complicated whole, the single entities are iterations of the whole (Gleick 162). Every single narrative unit within a serial drama is a reflection of the narrative as a whole, and together all of these units work together to drive the story forward in a compelling way.

While this narrative cohesion is determined, in part, by the underlying, self-similar, structures that I have outlined above, each individual serial drama also has its own unique set of parameters that informs and is informed by a TV show's tone. This is where the "differences of ambition, or execution, of style" that Goldberg and Rabkin mention come into play (13). The ambition, execution or style of a serial is determined by the specific and unique way in which a serial uses the repeated structures and patterns of TV drama narratives. Or, in other words, the creative decisions that TV writers make for their particular show are what constitute the unique parameters of a serial-not just that there 
must be a character, or set of characters, with a strong goal, but who the characters are, what it is they want exactly, and how these particular characters will go about getting it. Goldberg and Rabkin call this the "franchise":

A television series is the continuing adventures of a character, or a group of characters, setting out each week to achieve a predetermined goal [...] The pursuit of that goal, and the manner in which the characters do it, is the framework for telling stories. There's another word for that framework: It's the franchise of the series (14).

A successful serial drama will set up its "franchise" in the very first episode and must remain within the parameters of its own franchise for the rest of its run. For instance, if a show sets its characters up in a world that adheres to real-world logic then it must continue to adhere to real-world logic throughout the entire serial. If a show breaks real-world logic early on, it must remain consistent with the level at which real-world logic is broken. Epstein addresses the self-imposed boundaries of TV drama when he states that "[t]he essence of a successful TV show is consistency. Audiences tune in every week to see compelling stories told in a consistent way. They tune in each week to see the same thing" (17). Mittell discusses something similar when he explains that the pilot of a complex TV show teaches the audience how to watch the rest of the serial: "One of the challenges of any ongoing series is to find the balance between following its initial template and discovering itself through its ongoing development" (56). Dunleavy refers to this template or set of parameters of serial dramas when she discusses the 'concept' (or 'big idea') that fuels and locates their stories. She writes that dramas could hardly be more different from each other in terms of their 'concepts' (2).

The way in which a successful serial drama must sustain its own franchise and remain consistent can again be compared to Feigenbaum's examination and measurement of bifurcation points in a system that is on its way to chaos. When a slight change in a system causes it to shift in behaviour by doubling exponentially in value over time-in turn giving the appearance of irregular behaviour that is, in actuality, occurring at a constant rate-the bifurcations always converge towards the same value. While I made a comparison above between these converging points of bifurcations and the way ' $B$ ' and ' $C$ ' storylines converge 
with the ' $A$ ' story on a thematic and plot-level, the similarities can also be seen in a show's convergence of tone, style, and texture: its franchise.

The franchise of a serial drama is not something that is necessarily imposed from external forces - the parameters or boundaries of a show's tone or style are not something that can be drawn or marked out before a serial is written but are rather formed from within the writing process itself. This is similar to Feigenbaum's discovery that the constant rate at which points bifurcate occurs because of a deterministic relationship between the parameters of a system; such behaviour occurs because of some intrinsic aspect that exists within the system itself. A basic analogy of this intrinsic determinism can be found in the way that a standard pendulum clock works: its state of equilibrium is the regular swinging motion, and if a passer-by were to bump the clock, the pendulum's motion is likely to become temporarily erratic, but will eventually return to its equilibrium of the regular swinging motion again. But this is not to say that there is only one possible state of equilibrium for the pendulum -it could also be hanging straight down, without moving (Gleick 169-170). The same can be said for serial drama narratives. The presence of these sets of boundaries is essential for the success of any serial drama, and they function in the same way, but this isn't to say that every serial drama has the same set of boundaries.

A classic example of the self-imposed parameters of a serial drama's franchise is in the first episode of Lost (ABC, 2004-2010) when a polar bear turns up in the jungle. From this moment on, we know the show is not grounded in real-world logic, so when increasingly strange things start to happen as the show unfolds, the audience is more willing to go along with these events than if the show had set itself up as grounded in real-world logic. As Epstein writes:

TV is a constructed reality. The audience knows that, so they're willing to suspend their disbelief. But they'll only suspend so much. The more you have characters behaving in ways that don't match their reality-the more plotholes you have-the less invested they'll be in the characters and the story (170).

There are several examples of TV dramas that perforate their own boundaries, resulting in the writers subsequently trying to steer the story back into its original boundary. One 
example of this is a storyline that emerges in the premiere of the second season of Friday Night Lights (NBC, 2006-2011) wherein the character, Landry Clarke-the loveable nerdmurders a man who threatens to attack his love-interest, Tyra Collette. Together the pair dump the body in a river and try to forget the event ever happened. Several TV critics took issue with how this incident didn't fit into the mould of the show:

The second season premiere offered a plot development so wildly off-pitch that it could conceivably cast a pall over the series for the rest of its run (Marc Hirsh, today.com).

The principals don't disappoint, but there's a twist in the episode that is absurdly melodramatic and unbelievable, and will have enormous consequences. The plot thread could easily overwhelm the show and kill it (Nancy Franklin, The New Yorker).

Despite this departure from its parameters, however, the incident did not overwhelm and kill the show. After several episodes a guilt-ridden Landry eventually confesses the murder to his father, a policeman, who manages to get Landry and Tyra off without any charges. Once the storyline was apparently resolved, the show continued for another three seasons, never referring to the incident as if it was completely erased from the memories of the characters, and the writers. Here we can see the similarity between the parameters of a serial's franchise and the way in which points of bifurcation in a system on its way to chaos converge, or how a pendulum will return to its state of equilibrium after an external interference.

From these examples we can see that the clearest way to define a show's parameters is by identifying what doesn't fit within them; the parameters of a show only become visible when they are broken, something is included in the show that clearly does not belong there. This demonstrates the way in which serial dramas are comprised of structures and rules that must remain hidden beneath the surface of their complex stories if they are to succeed. If the rules of a serial are broken in some way or structures are exposed to a viewer, it is likely that the serial will fail, or will at least have to work hard to recover and return the serial to its state of equilibrium. Again, we can compare these hidden structures and rules to the way in which Feigenbaum's constant demonstrates that all systems that are on the path to apparent disorder exhibit the same underlying regularity within the individual system itself, 
as well as across a number of different and unconnected types of systems. The constraints and limitations that are repeated and embedded in all serial drama narratives - whether they be structural, stylistic, or tonal-are utilised in such a way as to create variation within the scope of a single serial drama, and also ensure that all individual serial dramas are unique to one another. I will now examine The Wire through the lens of "Universality" to demonstrate that the underlying rules and structures are present in all successful serial dramas.

\section{"Universality" and The Wire}

The Wire has been hailed as one of the most ground-breaking and complex examples of storytelling in TV drama. In fact, it has been said by many-including the show's creator, David Simon - that The Wire is not really TV at all but is more like a "visual novel" (Alvarez 23). Indeed, The Wire is unlike any TV show before it. Its narratives are slow-paced and sprawling; set ups are often left for several episodes, even seasons, before being paid off; central characters are introduced and discarded indiscriminately; characters and their circumstances rarely change; the narrative is never driven by mystery or intrigue; an extraordinary number of scenes simply depict characters in meetings, trying to get paperwork signed off. However, these aspects of the show do not necessarily mean that The Wire is not really TV or should be thought of as a visual novel. Arguments against labelling The Wire as a novel have cropped up in a handful of works that examine its narrative complexity. Jason Mittell argues that The Wire employs a new mode of televisual storytelling that he calls "serialized procedural", but which still exists within the realm of TV and adheres to TV narrative conventions (437). Linda Williams argues that The Wire uses its various narrative parts to produce a particular rhythm that is specific to TV narratives. Ted Nannicelli makes a similar argument, suggesting that The Wire arranges its various parts in a way that allows for in-depth character development that isn't possible in other narrative forms (198). Sebastian Armbrust argues that The Wire adheres to procedural narrative techniques that are not as visible as in most other shows, but that are still very much present in the show on a micro-narrative level (5). Just as I will be arguing, these authors all acknowledge that The Wire is indeed an innovative serial drama that subverts many of TV's 
conventions, but this innovation doesn't mean that the show abandons these fundamental conventions.

In this section I will examine The Wire through the lens of "Universality" to demonstrate that the complexity of serial dramas lies in the way the narrative form uses simple structures to produce creative and unique narratives that engage viewers. I will argue that The Wire uses the same simple structures as any other serial or series, but the way in which The Wire uses these structures is different than most; it is the execution, style, tone or "franchise" that is different from other serials, not the underlying narrative structures that the show employs. In order to argue this point, I will first examine the units and parts that comprise The Wire-specifically its episodic structure-and how they function in a similar way to the self-similarity that Feigenbaum discovered in his investigation of systems on their way to chaos. I will then examine the "franchise" of The Wire through the lens of Feigenbaum's constant as a way to demonstrate the regularity and consistency that is unique to The Wire and therefore sets it apart from other serials.

Despite its sprawling, overlapping and open-ended narratives The Wire employs the various narrative units that I discussed in the previous section in relation to "Universality". To reiterate, a serial is made up of seasons, which are made up of episodes, which are made up of acts, which are made up of scenes. These units have the same essential function of driving the ongoing story forward, while also providing a sense of narrative unity through their self-similarity. This function of each narrative unit in serial dramas can be compared to the scaling properties that were integral to Feigenbaum's understanding of dynamical systems that are on the path to chaos. These dynamical systems are made up of smaller single entities that not only work together and communicate in order to form a complex whole (like water boiling, or a metal becoming magnetised), but are actually iterations of the whole.

These narrative units can be seen in The Wire which, as a whole, examines the ambivalent and multifarious inner-workings of Baltimore City. Each of the five seasons that make up the serial explores a particular facet or institution within Baltimore City and its relationship with law enforcement: the illegal drug trade, the port of Baltimore, the city government and 
bureaucracy, the school system, and the print news media. Within each season we follow characters, or individuals, as they struggle with the various pitfalls of the particular institution that they exist within. In this sense, every unit of The Wire is some iteration of how the individual struggles to make progress or change within a broken system, as well as how the broken system fails to ever be fixed because of the conflicting agendas of the struggling individuals within it. Here we can see DiMaggio's equation which underpins every unit within a TV series or serial no matter how big or small (Character + Dramatic Need + Obstacles + Conflict $=$ Action) functioning in a self-generating way. That is, one character's need to survive within their system or institution often produces obstacles for another character or institution, and the ramifications of trying to overcome those obstacles produce further obstacles for others.

The Wire appears more fluid when we take a closer look at its structure at an episodic level. Most shows, even in serialised dramas, have some level of narrative cohesion on an episodic level. Individual episodes tend to have some sort of focus-a particular character arc, relationship, storyline, or conflict-that contributes to the larger, ongoing narrative but also has its own sense of conclusion or resolution that frames the episode itself. In Mittell's words, "The Wire takes a starkly different approach to its episodic structure, as there are almost no stand-alone plotlines within any given episode. All of the program's narrative events are either independent moments illustrating characters but lacking larger arc importance[...] or contributing to the slow accumulation of the central plotlines that run throughout a given season" (29). Indeed, in most episodes of The Wire, narrative threads that might be contained within an episode of any other serial drama are spread across several episodes so that by the end of any particular episode there are several storylines left open and seemingly unattended to. Nevertheless, I believe that beneath this fragmentation and open-endedness is some semblance of the TV act structure.

In order to examine this act structure in The Wire I will provide a close analysis of an episode that I have selected at random-episode eleven of season four ("A New Day"). I have identified seven storylines in this episode. Of these seven storylines, I see five as being fairly fleshed out (they have a sense of a beginning and a sense of an ending within the episode 
itself); the other two are simply a scene or two that function as seeds, planted for future storylines. The seven storylines, in order of significance (as I see them), are as follows:

Randy: Randy, a middle-schooler who was inadvertently embroiled in the murder of a drugdealer (Lex), learns that another dealer (Little Kevin) connected with Lex's death has also been murdered after Randy went to police with information. At school, Randy is beaten up by other kids who suspect him of being a police informant, which brings the whole ordeal to the attention of Randy's teacher, Prez, a former cop who goes to Sergeant Carver about it. Carver learns that Sergeant Herc has used Randy for his own benefit, rather than passing him on to Bunk who needed Randy's information for his homicide investigation into the case of several missing bodies. Ultimately, Herc gives Bunk and Freamon the testimony Randy gave him, which leads them to the discovery of several boarded-up vacant lots that Freamon suspects are being used as tombs for the missing bodies. As a result, Randy is left in an even more vulnerable position.

Carcetti: Mayor Carcetti deals with a complaint that has been made after Herc mistakenly attacked a well-respected Minister and is accused of racial profiling. Carcetti is advised to use Lt. Cedric Daniels, an African-American, as a way to deflect the accusations of racial profiling, which in turn creates jealousy amongst the commissioners of the Baltimore Police Department-Rawls and Burrell-who suspect the mayor to be grooming Daniels for commissioner. Meanwhile, Carcetti keeps up appearances by attending a fundraiser with corrupt political figures, making big promises to improve the police department, and giving contractors vague orders that result in them fixing minor issues around the city, only for his progress to be tarnished by news that the school system is running at a 54 million dollar deficit.

Bunny/ At-risk kids programme: Bunny is pleased with the progress that Namond, a middle schooler taking part in the newly formed school programme for at-risk kids, is making. Bunny's commitment to the programme is further strengthened when another kid in the programme lashes out, later revealing that he found his mother dead the previous night and was forced to attend school by his grandmother. So, when Bunny hears that the programme is being cut because of the school system's deficit, he is determined to keep it running.

Omar: Stick-up man, Omar Little, surveys the movements of Slim-a former criminal enforcer for Barksdale-who eventually leads Omar to the realisation that the drug kingpins, Prop Joe and Marlo Stanfield, are now working together. With this information, Omar threatens Prop Joe into helping him in his plan to rob Marlo.

Michael: Michael-middle-schooler-cum-hitman for Marlo-leads his peers in a revenge attack against a cop who has been picking on them for no particular reason. When Michael later brutally beats up the kids who were picking on Randy, Namond grows concerned about how hardened Michael is becoming. Meanwhile, Marlo's enforcers, Chris and Snoop, start to doubt Michael's loyalty to them when they hear he stood up for Randy, the suspected snitch. 
Bubs: Bubs is relieved when his friend, Sherrod finally returns after a short stint of drug dealing. Bubs and Sherrod try to take down the drug-addict who has been bullying and robbing Bubs, but it goes terribly wrong.

Dukie: Dukie, a promising middle-schooler, is disheartened to hear that he is going to be moved up a grade, into high school.

From this outline we can see there is a lot going on in the episode, but it is possible to see the act structure within each of the main storylines. That is, the act structure isn't spread across the episode as a whole, but is rather embedded in the individual narrative threads that make up the episode as a whole, therefore giving the episode its necessary shape and form in a more fragmented fashion. So, for example, the Randy storyline can be broken into acts, as follows:

\section{Act One}

Randy learns that Little Kevin is dead and he's likely going to be suspected of being a snitch because of it.

\section{Act Two}

Randy tries to go about his day as usual but is given the cold shoulder by other kids at school, intensifying his concern.

\section{Act Three}

Randy participates in the attack on the cop who has been picking on him and his friends, and is feeling secure having them on his side, but at school things get much worse and he is beaten by students accusing him of being a snitch.

\section{Act Four}

Prez learns of Randy's situation and promises to ensure he is protected. However, Randy is left in an even more vulnerable position when Randy's intel leads the police to the boarded-up vacant lots where the missing bodies are being stored.

While the resolution may not be quite as cathartic as the 'How to write TV' books instruct, we can see the general pattern of an four-act structure in Randy's storyline, which is, in turn, embedded in the larger storyline about Marlo Stanfield's missing bodies. Randy goes through a shift in circumstances throughout the episode due to the increasing level of jeopardy, and by the end of the episode we have a sense of the story seeing itself out, but a question is also posed, leading us into the next episode: what will happen to him once the bodies are found, and the information about the discovery goes public? 
While it's a little more convoluted, we can also see a similar kind of narrative pattern in Carcetti's storyline:

\section{Act One}

Carcetti is faced with the repercussions of Herc's brutality case, and realises how badly it could reflect on his Mayoralty if he doesn't deal with it in the right way.

\section{Act Two}

Carcetti puts Daniels in charge of dealing with Herc's punishment, and in the meantime focuses his attention on getting the Police Department to do real police work, rather than just "juking the stats". He also puts his plan in motion to fix minor issues around the city. Meanwhile, Burrell and Clay Davis conspire against Carcetti.

\section{Act Three}

Carcetti continues to make promises to improve police work, and is also happy to see that his plan to fix minor issues around the city has succeeded. But this small victory is interrupted when Burrell visits Carcetti, and convinces him that Daniels' punishment of Herc was too weak, and that he has the answers he needs.

\section{Act Four}

Carcetti faces a further blow when he hears that the school system is running at a $\$ 54$ million deficit, therefore hindering his plan to improve the state of the BPD; hinting at the possibility in the next episode of Carcetti having to choose between funding the school system or the police department.

Here, we can see that the jeopardy Carcetti faces is not building across the acts in quite the same way that it does in Randy's storyline. Instead, Carcetti is constantly faced with roadblocks that prevent him from making any progress, and results in his storyline being stuck in a sort of loop-one step forward, two steps back. This lack of narrative progression in his storyline can be seen as reflecting the intentions of the writers to represent the realistic struggle of a politician who is genuinely trying to make a difference but can't. While we don't see much of a change in Carcetti's circumstances in this episode, we can look at this episode as being a step in Carcetti's season-long character arc which eventually sees him attempting to become governor in the hopes of having more influence on how things are run. 
The other five storylines also adhere to an act structure, but since they are less prominent in the episode they are made up of three acts or less. We can think of these storylines in the same vein as $B$ and $C$ (and $D, E$ and $F$ ) storylines. The resolutions to these storylines are even more open-ended, due to the fact that they are the beginnings of storylines that will feature more prominently in future episodes, but-as I will outline in more detail below-these storylines are interlinked and converge in the same way that Feigenbaum's bifurcation points converge geometrically.

The structure in Bunny's storyline can be described as follows:

\section{Act One}

Bunny is pleased with the progress Namond is making in the programme, but this is undercut when another kid violently lashes out in class.

\section{Act Two}

Bunny learns that the kid who lashed out in class had found his dead mother the night before, thus showing Bunny the instability in these kids' home lives, and therefore strengthening his commitment to the programme.

\section{Act Three}

Bunny learns that the programme is going to be cut because of the school budget, and now has to fight to keep it running.

Omar's storyline follows a similar three-act structure:

\section{Act One}

Omar inconspicuously waits for Slim to show up, and follows him when he finally does.

\section{Act Two}

Omar learns that Marlo and Prop Joe are working together.

\section{Act Three}

Omar threatens Prop Joe into helping him in his revenge plan to rob Marlo.

And Michael's storyline can be broken down in this way:

\section{Act One}

Michael encourages his peers to make a revenge attack on the cop who has been hassling them. 


\section{Act Two}

Michael leads the revenge attack against the cop, and shows how menacing and brutal he can be. This hardening is reaffirmed when he beats up the kids who hassle Randy.

\section{Act Three}

Michael's loyalty is questioned by Chris and Snoop after they hear that he stuck up for Randy, the snitch, thus putting him in potential danger.

Bubs' storyline only has two acts, or since they're so small, we could call them beats:

\section{Act One}

Sherrod returns and gives Bubs new hope for his attempts to confront his bully.

\section{Act Two}

Sherrod and Bubs are defeated by Bubs' bully.

While Bubs' presence is very small in this episode, his storyline is significant when we look at the episodes surrounding this one. In the previous episode Bubs intentionally set Herc up to be accused of police brutality and racial profiling, as payback for Herc failing to help him confront his bully (and this turns into a huge problem for Carcetti, and ultimately results in the reshuffling of the police department's hierarchy). This is a prime example of how, due to the show's interconnectedness of all the storylines and narrative units, small events and storylines in The Wire often impact and converge with bigger storylines later on. A storyline can exist independently, and simultaneously be influencing and generating other storylines. We can see this in Randy's storyline as well-Randy is simply dealing with his personal problems in the best way he sees fit in the moment, but his individual actions have much larger consequences than he probably ever realises.

Dukie's tiny beat in this episode is also a significant storyline. Other than participating in the revenge attack on the cop, Dukie's only scene in this episode depicts him being told that he will be moving up a grade, into high school. Again, this is a seemingly minor event, but for Dukie this is the beginning of the end; he will be losing the security and support of his teacher and his peers, which ultimately leads to the homelessness and drug addiction that we see play out in season five where Dukie can be seen as taking the place of Bubs. We can 
also see Dukie's storyline as being caused by the failings of the school system, which in turn is a result of the $\$ 54$ million deficit that Carcetti is confronted with in this episode.

From this episode we can see that The Wire mostly uses the interconnectedness of the selfsimilar units of TV drama in a way that contributes to the larger, season-long narrative rather than attempting to produce a rigid episodic narrative structure. However, an episodic structure still exists within the show, it's just that its execution of this episodic structure is different from other shows. Jason Mittell writes that the individual episodes in The Wire are defined more by their notable tonal moments, than they are by narrative events of plot revelations, but:

This is not to suggest that episodes of The Wire are just random collections of character moments and unrelated narrative events that happen to fall in the narrative sequence of the program's plot arcs-individual episodes frequently feature thematic and character-related parallels across plots, and often an episode's unity comes more from its consistent mood and tone than from a contained story (30).

Here Mittell is describing the narrative unity that Epstein says is achieved by using the "rule of one"; no matter how complex or convoluted an episode of a serial drama might appear, there is always one thing that remains constant within it. Or, as Gerald Kasey puts it, in order to be relevant to the overarching story, each narrative unit within an episode must "flow into the main theme and become a vital part of it, like a stream joining and losing its own identity in a river" (96). In the episode, "A New Day", we can see this "rule of one" in the sense of vulnerability that each character or institution is experiencing by the episode's end, and the resulting uneasiness that these situations evoke. Randy, Bubs and Michael are all vulnerable to physical attack by separate parties, Carcetti's storyline leads to an exposure of the vulnerability of the education system, and as a result Bunny's programme is at risk of being shut down. While these narrative threads don't overtly converge on a plot level, they do converge tonally.

Because The Wire employs the self-similar narrative units of TV drama, this sense of vulnerability and uneasiness can also be said to be a reflection of the mood and tone of the entire show. The Wire's convergence of tone goes hand in hand with what Goldberg and 
Rabkin call a show's "franchise"; the way in which the repeated structures and patterns of TV drama are used consistently within a serial to produce original and diverse narratives. As I discussed in the previous section a show's "franchise" can be likened to Feigenbaum's constant, which proved that the points of bifurcation in a system that are on the path to chaos are not just repeating the same behaviour, but are repeating the same behaviour at a constant rate; the bifurcations always converge towards the same value which demonstrates that there is a deterministic relationship between the parameters of a system. If The Wire shifted between different styles, tones and executions of narrative structure within and between each episode then it could be argued that The Wire undermines its status as a successful TV show. But it doesn't; The Wire sticks to the tone, style and execution that it sets up at the start; it stays within its own bounds. The Wire demonstrates Epstein's point that audiences "[...] tune in each week to see the same thing" (17).

Broadly speaking, The Wire's "franchise" embraces realism and moral ambiguity and thus resists sentimentality and catharsis. Because of this specific framework, The Wire uses the conventional narrative units of TV drama in a way that reflects the messiness and ambiguity of real life, which is why its narrative structures are looser and more open-ended than most TV shows. In other words, because The Wire adheres to its tone and style of realism, its treatment of the narrative units within TV drama are inverted rather than abandoned. The narrative units that are usually arranged or constructed in a way that builds towards moments of relief, happiness, or some form of satisfaction for a TV show's characters and viewers, are arranged differently in The Wire so as to build towards moments of sadness and frustration. It's not that the characters in The Wire don't have goals and desires that they strive for, as is integral to the success of any serial drama, it's simply that the characters, more often than not, are unable to achieve their goals because this is what the show is about; this lack of progress and fulfilment, the failure of institutions to protect individuals, fits within the parameters of The Wire's "franchise". Just as Feigenbaum discovered that when the parameter values of a system reach a certain point the system will go through a series of bifurcations that appear complex and disordered, but are actually occurring at a constant rate, The Wire succeeds as a TV serial drama because it remains 
regular and consistent in terms of how its narrative threads unfold and progress in a sprawling and unconventional way.

We can see the parameters of The Wire's "franchise" being set up in the opening scene of the first episode of the serial. The opening scene depicts a crime scene, where we are introduced to McNulty as he speaks with a witness about the death of the victim, Snot Boogie, whose body remains sprawled out in front of them. Very quickly the show sets itself up as an unconventional crime show when the subtextual line of questioning between these two shifts from "who did it?" to "why did it happen?", and then-when the witness reveals that Snot was known to steal all the money after every craps game he ever played-"how did this not happen sooner?" The mundane tone in which McNulty asks his questions suggests that this kind of thing happens all the time, and the death of Snot Boogie is not shrouded in mystery or intrigue in the way that other crime shows would frame it. We know, from this moment, that the show is not going to be examining the mystery of an isolated incident, but rather the systemic issues that lead to the recurrence of such homicides. When McNulty asks the witness why Snot Boogie was allowed to continue to play craps when he was known to steal, he responds, "Got to. This America, man." And so, as has been observed by many critics, this scene contains the entire "DNA" of the serial as a whole. The "game" never ends because everyone is allowed to play, and not everyone has the same agenda. Because The Wire sets itself up as an atypical crime show that is grounded in realism, the serial goes on to consistently undermine the narrative conventions that are used in most crime shows. It is in the consistency of this undermining of the narrative conventions that the show also adheres to the conventions of serial drama narratives.

Just as Feigenbaum discovered regularity and consistency beneath systems that are on the path to chaos, The Wire has a self-imposed boundary that shapes and regulates the narrative units within the serial even though it might appear unconstrained and highly complex on the surface. As I've outlined above, we can see that The Wire's boundary and shape is formed from its consistently realistic style and tone. But, in a way, we can also see its overall shape forming from its very ambition to be unlike any other TV show. The show creators intended The Wire to be different, so they set their parameters in ways that would challenge our expectations of what a serial can do, on both a structural and thematic level. 


\section{Conclusion}

Feigenbaum's discovery of "Universality" meant that difficult problems could be solved with simple and efficient solutions, and that these simple and efficient solutions could be applied to systems that appeared to behave in vastly different ways. "Universality" meant that the otherwise obscure behaviour of a system en route to chaos could be measured and examined in a way that wasn't possible before. In a similar way, examining the simplicity behind the complexity of serial dramas through the lens of "Universality" is useful for demonstrating how to understand the essence of an entire serial by isolating and examining its smaller fractal-like parts. "Universality" shows us that, no matter how complex or different one serial drama might appear compared to another serial, all serials are fundamentally the same. In this sense, examining serial drama narratives through "Universality" accentuates the idea that the complexity of serials is achieved through the repetition of simple, rigid structures. Furthermore, rather than examining a particular serial drama as being more complex or simpler than another serial drama, "Universality" allows us to examine the interconnectedness of simplicity and complexity in serial dramas. All serial dramas are simple and complex at the same time, the difference is in how a particular serial utilises this interconnected relationship between simplicity and complexity. This is because the complexity of a TV serial can be read as being very simple, and the simple, repeated structures of serial narratives can be read as functioning in a complex way. And so, "Universality" shows us that the simplicity of a serial drama not only enables its complexity, but it can be thought of as a defining characteristic of its complexity. In the same way that systems are deemed chaotic due to the intricate intertwining of stability and instabilityrather than the complete disorder and confusion that the term is commonly associated with-serial dramas are complex, not because they are purely random and confusing, but because their sprawling and challenging narratives are underpinned by simple, repeated narrative structures.

Similarly, understanding the simplicity underneath the complexity of serial dramas through the lens of "Universality" is useful for someone who might be new to writing for TV. That is, a beginning writer can use "Universality" as a reference for how to manage the overwhelming number of narrative components that is required for a serial drama by 
approaching the story as a single simple formula that gets repeated over and over again in different ways and at different scales. By understanding the simple rules and structures of TV serials through the lens of "Universality", a writer can push the boundaries of the narrative form, and let the story unfold in unexpected ways with the guidance and security of the inherent rules and structures. "Universality" shows us that we can construct serial dramas in endlessly different and innovative ways, yet still fundamentally be the same. In the following chapter I will use strange attractors to examine how this dynamic between simplicity and complexity means that the narratives in successful serial dramas are unpredictable but also expected. 



\title{
CHAPTER TWO Strange Attractors and Unpredictability in TV Drama Narratives
}

\section{Introduction}

Kristin Thompson argues that the incidents in TV narratives that have the greatest effect are ones that occur unexpectedly or by chance, but also have an appearance of design, a kind of controlled coincidence, or patterned randomness:

\begin{abstract}
Such incidents have the greatest effect on the mind when they occur unexpectedly and at the same time in consequence of one another, there is more of the marvellous in them than if they happened by themselves or by mere chance; even matters of mere chance seem most marvellous if there is an appearance of design, as it were, in them (38).
\end{abstract}

The dynamic between simplicity and complexity that I discussed in the previous chapter means that a serial drama can arrange its parts to give the appearance of randomness so that its narrative is unpredictable and surprising but still expected. When watching a successful serial drama, a viewer can be familiar with the rhythm and formulae that underpin it, but this doesn't mean that they will know what will actually happen in the story, or how it will end. A viewer can also know the tone in which the story will be told but, again, this doesn't mean that a viewer will know how the story itself will unfold. In this chapter I am going to use strange attractors as a lens through which to examine the various ways in which serial dramas are able to be both expected yet surprising, predictable yet unpredictable. To reiterate, when a strange attractor is depicted in phase space, the curve of the attractor repeatedly forms loops and spirals without ever actually intersecting itself. Eventually, after numerous iterations, the curve of the strange attractor forms a distinctive shape or pattern.

An observer of such a system can make a guess as to the shape or path of an individual loop based on the shape of the loop before it, but there is no way of predicting what shape the curve will form in the long term due to small changes having large effects. In other words, a strange attractor demonstrates how a chaotic system is both stable and unstable, ordered and disordered, and therefore unpredictable. In a similar way, a serial's narrative as a whole 
can be thought of as being made up of smaller self-contained narratives that work together to form the larger, ongoing narrative. These smaller, self-contained narratives within a successful serial drama might give viewers clues and hints as to the direction of the serial in the short term, but a viewer shouldn't be able to predict the exact ending based on these self-contained narratives.

I will begin this chapter with an introduction to strange attractors, before applying the properties of strange attractors to my analysis of how the various narrative units within a serial drama are arranged to produce predictable yet unpredictable stories. I will finish the chapter by providing an analysis of The Leftovers that will be framed by the points that I draw from my comparison of serial drama narratives to strange attractors.

\section{Strange Attractors}

An attractor refers to a set of states-in finite dimensional space-towards which neighbouring states are drawn or tend to evolve. If a system tends to settle to a particular point regardless of the system's initial conditions, an attractor must be present in that system. A swinging pendulum, for instance, has two variables-its position and its velocitywhich change continuously if the pendulum is swinging without the interference of friction. If mapped in phase space the frictionless pendulum's movements would be represented by a line of points that loop around and around forever. If friction is added to the mix, then the image changes: "[e]very orbit must eventually end at the same place, the centre: position 0 , velocity 0" (Gleick 134). This central point "attracts" the orbits, and instead of going around and around forever in phase space, the orbits spiral inward (134). If another variable is added to the swinging pendulum - such as a spring that gives the pendulum energy while it loses the energy through friction - the system will likely settle into a stable orbit: "[n]o matter where the pendulum starts, it will settle into that one orbit" (138). Unless, of course, the spring is producing a low amount of energy in which case, the pendulum will eventually settle to a stop. This example of the pendulum demonstrates how a dynamical system tends to have one of two types of attractors, depending on its variables. One of these attractors is referred to as a "Fixed Point", which results in the long-term motion of a dynamical system staying "at rest in a steady state" (Stewart 99). The other type of attractor is referred to as a 
"Limit Cycle", which means that a system will repeat some series of motions periodically (99). In lan Stewart's words, a dynamical system with an attractor will either "sit still or go round and round" (99).

There are, however, dynamical systems that don't have a fixed point or limit cycle-systems that exhibit both stability and instability. Turbulence, for instance, never produces "any single rhythm to the exclusion of others" (Gleick 138). According to Lev Landau, a Russian scientist who specialised in fluid dynamics, the onset of turbulence could be pictured as a "piling up of competing rhythms". When more energy comes into a system "new frequencies begin one at a time, each incompatible with the last" (Gleick 124). The general scientific understanding of turbulence is that when flow is smooth, small disturbances dissipate. But when turbulence sets in, disturbances grow catastrophically like the bifurcation points on Feigenbaum's logistic map, discussed in the previous chapter. Turbulence is order and disorder intertwined (138). David Ruelle and Floris Takens, also specialists in fluid dynamics, wanted to delve further than this general understanding of turbulence and attempt to answer the elusive question of what actually happens when fluid changes from smooth to turbulent. They wondered whether there was some kind of attractor within turbulence, despite its instability.

Ruelle knew that if an attractor was present in a turbulent system, it could not be a fixed point attractor, because energy was entering the system as well as draining out of it, which meant that the flow would never come to a stop (138). But the properties of turbulence also did not align with the properties of a system with a limit cycle attractor since its behaviour never repeated in exactly the same way and is better explained as an accumulation of independent motions. So, Ruelle posited, there must be some other kind of attractor in turbulence-one that was stable, low-dimensional, and non-periodic all at once. Ruelle was searching for a phase space curve that could be:

[...] drawn in limited space so that it would never repeat itself and never cross itself-because once a system returns to a state it has been in before, it thereafter must follow the same path. To produce every rhythm, the orbit would have to be an infinitely long line in a finite area (Gleick 139). 
Ruelle's and Takens' ideas were not embraced by other scientists at the time, but their hypotheses were later connected to the discovery of the strange attractor whose curve, when depicted in phase space, comes repeatedly close to intersecting itself but never quite succeeds. As I discussed in the introduction to this thesis, it turned out that such an attractor had been explored earlier by Edward Lorenz in his 1963 paper on deterministic chaos, in which he provided a drawing of what he termed the "Lorenz attractor" (see figure 2). Lorenz's drawing of the Lorenz attractor was first used to demonstrate the unpredictable behaviour of his waterwheel, which he later realised could also be used to demonstrate weather patterns that repeated but never in the exact same way: "Those loops and spirals were infinitely deep, never quite joining, never intersecting. Yet they stayed inside a finite space, confined by a box" (Gleick 140).

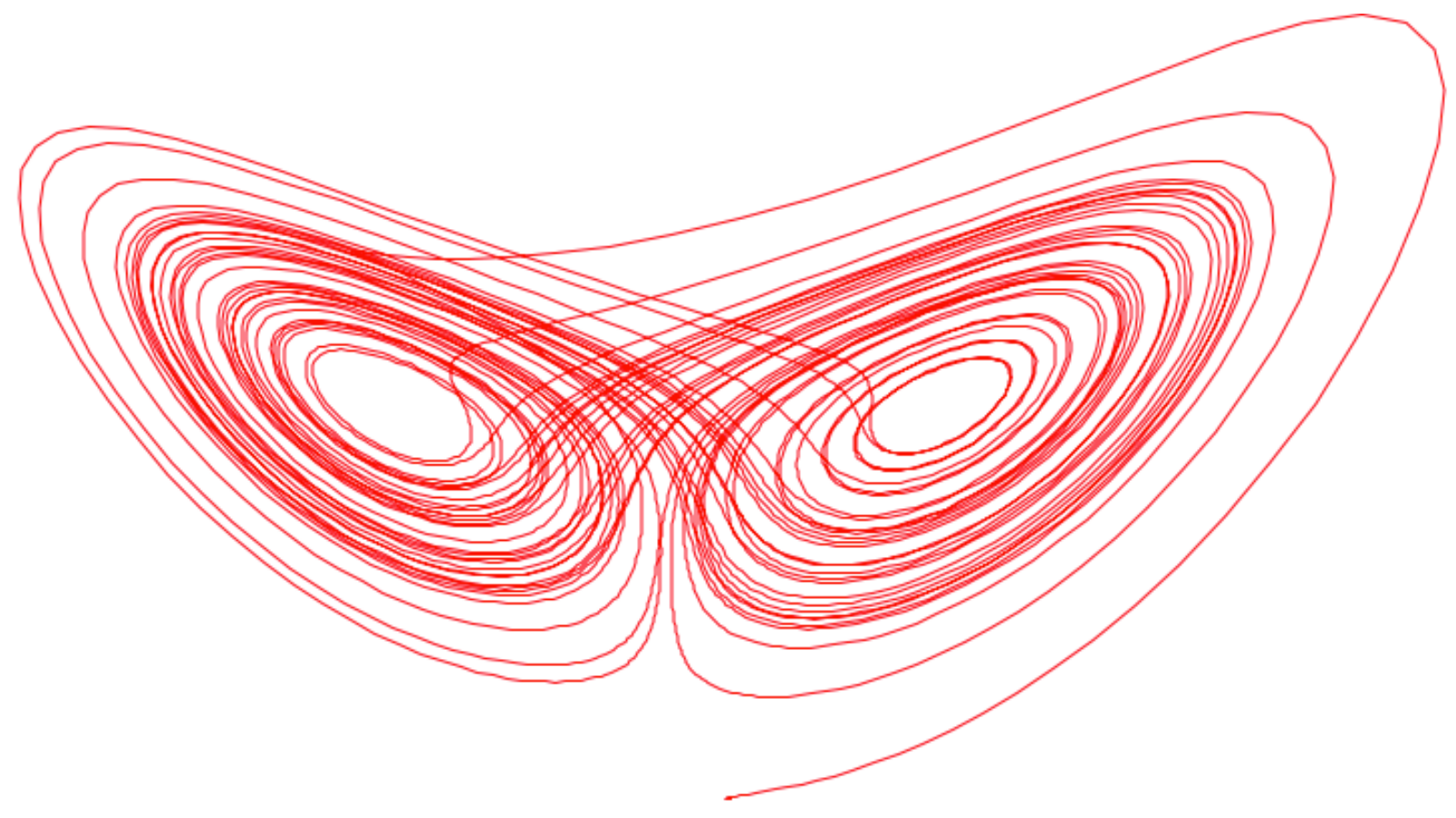

Figure 2: The LORENZ ATTRACTOR maps the phase space curve which never intersects itself: "Instead it loops around and around forever" (Gleick, 29), image retrieved from: Wikimedia Commons (Cepheus, 2 Oct. 2006), <commons. wikimedia.org/w/index. php?curid=1241516>

Lorenz was left wondering how it could be possible for two surfaces on his attractor to merge, while any two trajectories on his attractor were unable to merge (Gleick 140). Lorenz was posing this question before Mandelbrot's images of fractals, but the answer that he provided to his own question was that his attractor was fractal in nature-it possessed self-similar structures across different scales. Lorenz realised that "[w]here the spiral appears to join, the surfaces must divide [...] we see that each surface is really a pair of 
surfaces, so that, where they appear to merge, there are really four surfaces" and so on until "we finally conclude that there is an infinite complex of surfaces" (141). If one were to attempt to make long-term predictions of a system that contains a strange attractor, or any chaotic system, by stringing together short-term predictions, small irregularities would gradually build up, until the predictions turned into total nonsense (Stewart 127). The fractal properties of Lorenz's system explains why small events can have big effects, also known as the "butterfly effect." As Stewart points out, there is not just one butterfly flapping its wings but a chain of interconnected "butterflies" in the system that interfere and contribute to the long-term events:

By definition a hurricane is a storm of a certain size. But the definition is imposed by people on nature. In reality, atmospheric scientists are realising that tumult in the air forms a continuum, from the gusty swirling of litter on a city street corner to the vast cyclonic systems visible from space (Gleick 108).

The "infinite complex" of self-similar surfaces displayed in Lorenz's weather system, show us how the small disturbances caused by the butterfly flapping its wings can, in a sense, be considered as much a part of the hurricane as the hurricane itself: "[t]he ends of the continuum are of a piece with the middle" (108).

Not only does the presence of strange attractors demonstrate that long-term predictions are impossible in chaotic systems, it is also near impossible to understand and observe the inner-workings of a strange attractor. This difficulty is due to the fact that phase space curve of the system produces a complex web of intersecting points through three dimensions or more which, when examined from the outside, could look like "a dark scribble in space" with an internal structure that seems incomprehensible (Gleick 142). A solution to this problem was found in what is termed a "return map" or a "Poincaré map", which essentially involves removing a dimension from an attractor, and sampling the state of a system at intervals rather than observing the system as a continuous line. This process meant that scientists could see the points where an orbit passes through a place of intersection, and they found that the points that mark some regular time interval are either random or they begin to form a shape (142). Ian Stewart describes this process as slicing an attractor, and retrieving the points where the trajectory hits the line slicing it, demarcating the points of first return: "[i]f you iterate the first-return mapping, you get the first return, then the 
second, then the third...you're sampling the full dynamics at regular intervals of time" (Stewart 104).

Michel Hénon, and astronomer, sought to find a way to further simplify the return map for his investigation into the many-body problem. By marking on a flat piece of paper the points where a celestial orbit would cross a particular plane, and by tracing the movement of that point from orbit to orbit, Hénon discovered that orbits would almost, but not quite, repeat: "the successive points actually made a circuit around the curve, but since they do not come around to exactly the same place, eventually, after hundreds and thousands of points, the curve is solidly outlined" (Gleick 147).

14 years later, when Hénon shifted his focus to discrete time rather than continuous flow, he formulated difference equations that produced results that were similar to those found in Stephen Smale's Horseshoe Map, which-as I outlined in my introduction-took the Poincaré section and worked backwards, forming iterations from a series of "stretching and folding". That is, a system's phase space would be stretched so that nearby points are torn apart. And then, since the phase space is bounded or finite, "there isn't room to stretch everything. It gets folded round itself many times [...]" (Stewart 134). This means that even though nearby points are torn apart, some points that are far apart end up moving closer together (134). Yet, while it is clear that such a process of stretching and folding moves points closer together and tears others apart, it is very difficult to predict where the next point will appear-except that it will be somewhere on the attractor (Gleick 150).

Indeed, Hénon's equation also produced an iterative "stretch-and-fold" process with similar results. In Gleick's words, "[t]he key, he believed, was the repeated stretching and folding of phase space in the manner of a pastry chef who rolls the dough, folds it, rolls it out again, folds it, creating a structure that will eventually be a sheaf of thin layers (Gleick 149). Upon magnification, lines on his map that appear to be thick, are revealed to be two distinct lines, then two lines become four lines, and each of the four lines are revealed to be made up of two more lines, so on in the same way that Lorenz's attractor displays infinite regress (Gleick 150). 
The regression of lines attributed to the Hénon attractor also displays a more specific type of fractal structure known as the Cantor set (see figure 3). Lorenz describes the Cantor set:

Take a horizontal line segment, discard the middle third while retaining the end points, then discard the middle third of each of the two resulting segments while again retaining the end points, and continue the process to infinity. It might seem that in the limit nothing would be left but end points, but this is not the case. The point one-fourth of the way in from the left end of the original segments, for example, will, after the first step, be one-fourth of the way in from the right end of the left segment, and, after each succeeding step, will be onefourth of the way in from one end or the other of the segment in which it lies. Thus, it will never be an end point, yet it will never be discarded (Lorenz 150).

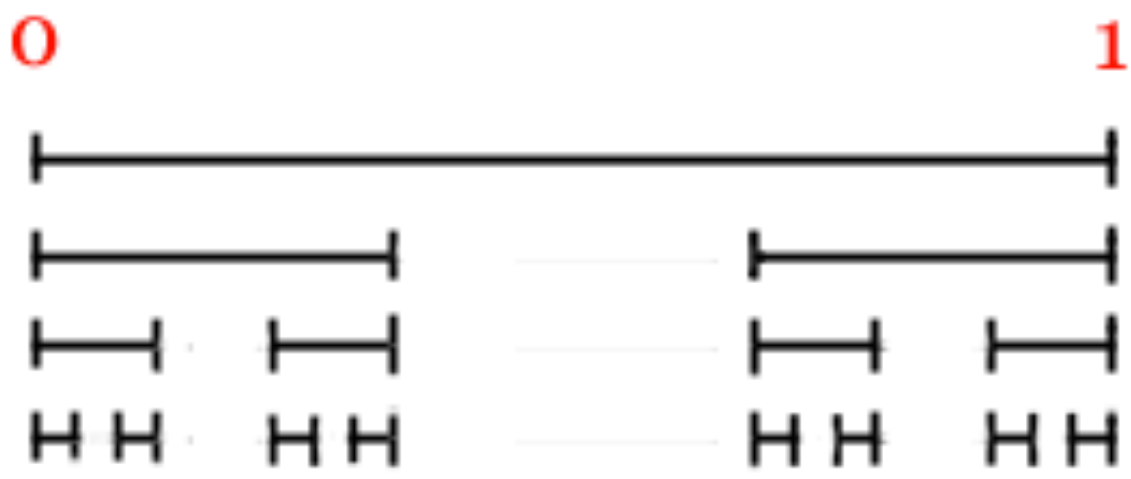

Figure 3: The first four intervals of a CANTOR SET, image retrieved from: GutiérrezNaranjo, Miguel \& Mario Pérez-Jiménez, "Fractals and P Systems" 2015, p. 73; Research Gate, <researchgate.net/publication/265994686>

After several intervals one is left with an array of points called "Cantor dust", which are infinitely many, yet their total length is 0: "A strange 'dust' of points, arranged in clusters, infinitely many yet infinitely sparse" (Gleick 92). When the final form of Hénon's attractor is viewed through progressive magnification one will see the unfolding "nested detail, lines within lines" (15). But the effect of such a strange attractor can also be viewed in reverse as an eerie shape emerges out of the points over time (150).

While a group of information theorists, led by Robert Shaw, would later go on to attach numbers to the chaotic behaviour that strange attractors exhibit, all investigations into 
strange attractors were always based on the abstraction, simplification and illustrations of dynamical systems. What these abstractions and illustrations demonstrated is that "[d]isorder was channelled, it seemed, into patterns with some common underlying theme" (Gleick 152). Strange attractors illustrated the intricate intertwining of stability and instability, order and disorder, in a chaotic system that meant that its behaviour was unpredictable yet deterministic (153).

In the following section I will use the intertwining order and disorder illustrated by strange attractors to look more specifically at how the structures that underpin a successful serial drama are used to generate narratives that are both predictable and unpredictable. First, I will compare the disorder and order that exists within a system that is pulled towards a strange attractor to how most successful contemporary TV dramas employ a series-serial hybrid narrative form that is continuous but made up of self-contained narratives and are used to push and pull at a viewer's expectations. I will then use the fractal properties of the Poincaré section technique as a way to argue that, because of its hybrid form, a successful serial drama is an accumulation of end points that eventually form the shape of the entire serial but do not mean a viewer can predict how a serial will end.

\section{Strange Attractors and Unpredictability in TV Drama Narratives}

As I have mentioned previously, a particular point that crops up in many discussions surrounding the recent developments in TV drama narratives is the distinction, or lack of distinction, between a TV serial and a TV series. To reiterate, a series uses self-contained episodic narrative strategies so that a storyline is developed and fully resolved within the space of a commercial hour, and a viewer doesn't necessarily need to watch episodes in order (Dunleavy, 101). A serial, on the other hand, employs continuous or ongoing narratives, which means that a viewer does need to watch episodes in order (102-103). This distinction between serials and series, can be thought of in the same way that fluid dynamists understand the distinction between a regular attractor and a strange attractor. $\mathrm{A}$ system containing a regular attractor will follow one of two trajectories: a limit cycle, in which a system settles into a stable orbit; or a fixed point, in which a system will settle to a stop. A system that has a regular attractor present will settle into either one of these two 
states of equilibrium, in the same way that an episode in a traditional TV series will regularly resolve its storyline before the next episode begins. A TV series continually returns to the same point, before starting the same essential process, or narrative trajectory, again.

A serialised TV narrative, on the other hand, displays behaviour similar to that of a chaotic system that is pulled towards a strange attractor and exhibits an intricate intertwining of order and disorder. That is, the repeated loops of a strange attractor's curve that never quite intersect or repeat are similar to how serialised storylines are on-going and continuous, never repeating themselves nor settling down to a definitive end point (until the series finale, that is). Yet, despite a serial's continuous nature, its narratives are punctuated with endings - ends of acts, ends of episodes, ends of seasons, and the end of the serial. In this sense, a serial is more complex than simply being a purely open-ended narrative with a linear progression. Just as a strange attractor cannot be considered to be the complete opposite to a regular attractor in that it displays both order and disorder at once, a serial cannot be considered the complete opposite to a series. In fact, as I mentioned in my introduction, many scholars who examine the complexity of TV drama narratives agree that most contemporary TV serials should be considered a hybrid form that combines self-contained storylines and continuing storylines. Dunleavy, for instance, acknowledges that there are blended forms of these two TV narrative strategies. One of these she terms the "serialized-series", which refers to TV drama narratives that incorporate serial threads and arcs into the framework of a series problematic. Another form is the "series-serial", which refers to dramas that use progressive overarching stories but also incorporates a "series-like problematic" on a conceptual level to provide a flow of additional stories and one-off characters (103). For a serial drama to be successful, even if it mostly consists of ongoing storylines, its narrative should also contain smaller narratives within it, and as a result a serial can be seen as being both expected and unpredictable at once.

The Sopranos (HBO, 1999-2007) is a good example of a successful serial drama that displays this hybridity between the series and serial. In most episodes of the show, there is a selfcontained story that is resolved before the hour's end. Yet, such self-contained stories are often framed by season-long, or series-long, storylines. These self-contained stories may even contribute to the progression of the overarching narrative in some minor way, such as 
functioning as a turning point for a character. A strong example of this is the critically acclaimed episode in the third season of The Sopranos titled "Pine Barrens." In this episode Meadow Soprano's relationship with the son of a recently-deceased mobster hits shaky ground, and Tony comes to realise that his new relationship with a mistress closely resembles his relationship with his mother. These two storylines are continuations of storylines from previous episodes, and are also developed further in the episodes that follow. The third main storyline in this episode, however, follows Paulie and Christopher who wind up having to dispose of a body after what was meant to be a simple exchange of money goes horribly wrong. When the two venture into the snowy woods to dispose of the body they discover that the victim is still alive. After witnessing the victim escaping into the woods Paulie and Christopher fail to track him down, and they find themselves in a life-ordeath scenario that is different from the life-or-death scenario that the show usually portrays. In the end, Tony comes to Paulie and Christopher's rescue. While the mystery of the victim's whereabouts is left unanswered, this storyline reaches a satisfying conclusion by the end of the episode because its emotional core is tied to the relationship between Paulie and Christopher, and the driving question of whether they can survive in the freezing wilderness together. And, while it subtly feeds into the ongoing narrative of Paulie and Christopher's growing frustration with Tony's leadership, this is a stand-alone storyline that is contained within the episode.

The Poincaré map, or return map, which I outlined in the previous section, is a useful tool to understand how and why self-contained narratives-such as "Pine Barrens" - exist within the longer continuous narrative, especially with the advent of streaming services which have eliminated the need to cater to commercial breaks and even episode breaks. To reiterate, the return map involves removing a dimension from an attractor and sampling a system at intervals, rather than observing the system as a continuous line (Gleick 142; Stewart 104). The map meant that scientists could view an otherwise incomprehensible system as a series of distinctive points where a trajectory passes through a place of nearintersection. This ability to view a system as a series of distinctive points, is similar to how the distinctive self-contained narratives within a serial drama allow for more comprehensive and watchable TV. In "Pine Barrens", for instance, we can see that contained narratives exist so that a character or characters are given a goal that is specific to that episode, and the 
viewer is given an answer by the hour's end as to whether that character has achieved their goal or not. All the while, the overarching narrative questions remain unanswered and therefore continue to drive the main storylines forward. In other words, self-contained narratives provide a viewer with a satisfying parcel of information, so that they are content and excited to continue watching the serial even though they might not know where the larger, ongoing narrative is going exactly.

Not only does the return map reflect the momentary clarification and gratification that distinctive self-contained narratives provide within an ongoing narrative, but the points on the return map themselves are a useful way to reframe our understanding of how this hybrid form of TV drama narratives enables a serial to maintain an unpredictable narrative without losing its sense of coherence and progression. The points of intersection on the return map illustrate the combination of order and disorder of a system being pulled towards a strange attractor, in the way that the points appear scattered and random at first, but eventually, after several orbits, form a shape. The overall shape, or narrative, of a serial drama need not be achieved by providing storylines that constantly and directly progress the overarching storyline, but can be formed by taking diversions, even if these seem random and unrelated at first. When isolating the points, or episodes, they clearly function in their own right, but they also contribute to the overall "shape" and texture of the serial as a whole: "[s]ince they do not come around to the exact same place, eventually, after hundreds of thousands of points, the curve is solidly outlined" (Gleick 147). Gleick's description here again reflects the hybrid structure of the TV serial in the way that episodic or procedural elements may give the appearance of periodicity-the narrative coming full circle by the time the episode is up-when, in fact, a serial's narrative is always moving in a new direction. An episode might end definitively at one point, but the next episode may start at a completely different point in the narrative. Yet, each stop or start throughout a serial drama is contributing to the overall shape and texture of the narrative as a whole.

However, a TV serial's treatment of its self-contained narratives, or its intermittent stops and starts, can mean the difference between narrative confusion and narrative mystery, or the difference between a satisfied viewer and a frustrated viewer. As I discussed in the previous chapter in terms of the "rule of one", the diversions that come in the form of self- 
contained narratives can't be too unexpected or seem extraneous to the main story, or a viewer will feel the incidents in the story are happening at random, and will likely become confused and frustrated as a result. Here we can see the similarity of TV drama's selfcontained narratives to the way in which Gleick describes the trajectories of a Lorenz attractor: "[t]hose loops and spirals were infinitely deep, never quite joining, never intersecting. Yet they stayed inside a finite space, confined by a box" (Gleick 140). Selfcontained narratives can diverge from the core of the serial drama, but there is a limit to the degree to which it can diverge before the cohesion of the serial falls apart and a viewer will feel that the narrative is unfolding in a random way. To turn to Kristin Thompson's point about patterned randomness, again, incidents in the narrative:

"[...] have the greatest effect on the mind when they occur unexpectedly and at the same time in consequence of one another, there is more of the marvellous in them than if they happened by themselves or by mere chance; even matters of mere chance seem most marvellous if there is an appearance of design, as it were, in them" (38).

In this sense, a serial drama that is successful at providing incidents that occur unexpectedly but with the appearance of design can be thought of in the same way as the deterministic but unpredictable behaviour of a system that is being pulled towards a strange attractor. The self-contained narrative trajectories in a serial drama should never repeat in exactly the same way, but they also shouldn't appear to be completely random either. Lost, for example, is often criticised for its long-winded and digressive narrative, which could be said to be the result of the fact that it sometimes failed to provide stories that were simultaneously unpredictable but expected. Audience dissatisfaction arose from the narrative going in unexpected directions, but in a way that didn't have the appearance of design. Jason Mittell explains that Lost has its narrative rely on the fact that all of the pieces will eventually fall into place to provide a unified text: "[...] viewers watch Lost with a mind toward the totality of the series, working to assemble each segment into a unified narrative that will not be fulfilled for years to come" (11). Mittell argues that if the narrative appeared to be veering away from this totality fans lost faith in the show because underlying doubts were "often triggered by a sense of disunity stemming from the fear that the show is 'made up as it goes along', rather than carefully planned out in advance" (12). Indeed, Lost's series creator, Damon Lindelof, has had to defend his narrative decisions on numerous occasions, 
and explains that the divergent nature of particular narratives was, in part, the result of the network executives insisting that the writers commit to six seasons of story-to milk the success of its first season-when Lindelof and his writers already had an ending in mind that would not require an entire six seasons to reach. The writers therefore had to come up with narratives that could pad the serial out before it came to its intended conclusion, and as a result the storylines are often seen to drag and veer off in unpredictable and unnecessary directions in the show's middle seasons. Lindelof cites his least favourite episode as being episode 9 of season 3, titled "Stranger in a Strange Land", which tells the story of how Jack got one of his tattoos-a storyline that was completely tangential, adding nothing to the larger narrative (O’Neal).

We can see the difference between successful employment of self-contained TV drama narratives and unsuccessful employment of self-contained TV drama narratives by comparing Paulie and Christopher's storyline in The Sopranos episode, "Pine Barrens" and Jack's tattoo storyline in Lost's "Stranger in a Strange Land". In "Pine Barrens" the selfcontained narrative diverges from, but also feels like a necessary part of, the ongoing narrative. Whereas in "Stranger in a Strange Land" the self-contained narrative diverges from the main narrative and doesn't feel like a necessary part of it. The difference between a self-contained narrative that doesn't contribute to the ongoing narrative in some way versus a self-contained narrative that does contribute to the ongoing narrative is like the difference between a dynamical system that is unstable versus a system that is both stable and unstable. In other words, a narrative trajectory that deviates from the main ongoing storyline can be conceptualised in the same way as a dynamical system going through a process of folding and stretching when a strange attractor is present. A system's phase space would be stretched so that nearby points are torn apart. And then, since the phase space is bounded or finite, "there isn't room to stretch everything. It gets folded round itself many times [...]" (Stewart 134). This means that even though nearby points are torn apart, some points that are far apart end up moving closer together (134). Yet, while it is clear that such a process of stretching and folding moves points closer together and tears others apart, it is very difficult to predict which points will do so: “[y]ou can't see what's coming in advance" (134). While it is impossible to see what's going to happen in advance, it is knowable that whatever does happen will occur within the bounds of the system; the points 
will be somewhere near the strange attractor. In a similar way, the self-contained narratives of a serial drama will diverge from the main storyline but will do so in a way that gives the appearance of design and feeds into viewer's expectations; all these narratives must contribute in some way to the direction and shape of the ongoing narrative of the serial as a whole.

To help clarify this point we can think of the accumulation of endings of serial dramas in the same way that the points of intersection on the return map display fractal properties upon progressive magnification. Where the trajectory "appears to join, the surfaces must divide [...] we see that each surface is really a pair of surfaces, so that, where they appear to merge, there are really four surfaces" and so on until "we finally conclude that there is an infinite complex of surfaces" (141). In turn, this self-similarity means that such a system displays sensitive dependence on initial conditions (small changes in the system can have big effects down the line), but these initial conditions don't necessarily exist at the starting point of the system - the initial conditions are scattered throughout the system, producing a kind of continuum that means an observer can draw conclusions about the nature of the system by analysing any point on it. This notion that the end points of the continuum are "of a piece with the middle" is evident in the Cantor set, which Hénon discovered in his investigation of strange attractors in celestial systems.

The fractal surfaces that occur due to the presence of a Hénon attractor are regressive-one third of the middle of the beginning segment is removed, and a third of the middle of both the remaining segments is removed, and so on. The Cantor set is an accumulation or "continuum" of end points. But each new end point contains its own middle section that, when removed, produces new end points. The Cantor set, or continuum of end points, reflects the way the end points in serial drama narratives can also be thought of as starting points. And starting points and end points, can also be thought of as mid-points. Because of this "continuum", the set up to the pay-off of a particular storyline in a serial won't necessarily be located at the beginning of the serial, season, episode or even act. In fact, a set up could be found in the middle or end of another storyline. 
In turn, this means that for a serial to be successful an audience shouldn't be able to predict how a serial, or a self-contained narrative within the serial, will end based on how a storyline is set up at its beginning, but the general shape that will be formed once the end point is reached can be determined by examining how a serial treats its end-points at various scales; if a serial tends to rely on smaller, self-contained narratives then the longterm endings are likely going to be closed off, whereas if a serial relies more on an openended continuous narrative its long-term endings are also going to be open-ended. In other words, the fractal-like nature of end-points within a serial drama means that its finale may be unpredictable but there are also certain expectations that the serial must meet.

In an article on Vox.com, Todd VanDerWerff examines the spectrum of series finales and suggests that what qualifies as closure for every show is different, depending on the nature of the show itself. He quotes Joel Fields, the co-showrunner of The Americans (FX, 2013-2018):

Great endings fulfil the unspoken promise of the show, which is to say they leave you in a place where you feel finally satisfied, even if the satisfaction is about wanting more, or about feeling sadness for the characters, or feeling disquiet [...] It's about feeling dramatically satisfied and feeling some level of cathartic relief (qtd. in VanDerWerff).

So, as VanDerWerff suggests, the type of narrative closure that a TV serial employs in its finale depends on the way in which a serial has treated its narrative questions and mysteries throughout the serial. The Wire, for example, ends with an open-ended narrative that reflects its sprawling and open-ended narratives that I discussed in the previous chapter. We see where all the main characters end up, but these revelations are framed in such a way that suggests nothing has changed or resolved itself: Bubs finds redemption and resolution, but we see Dukie taking his place; Omar is killed, but Michael is likely going to fill his role. The serial ends, but the "game" is never over. The series finale of The Sopranos polarised viewers with its ambiguous cut to black while the Soprano family are shown eating at a diner, and before we see whether the person who enters the restaurant is Meadow Soprano or someone who is going to kill Tony. Such an ending could be said to reflect the way in which the serial as a whole would often use the uncertainty of who can and can't be trusted, as well as the uncertainty of if or when a character might be killed, to create 
dramatic moments and to drive the narrative forward. But it could also be said that this series finale departs from the way the The Sopranos treats its self-contained narratives which were usually closed-off and resolved, as is the case with the emotional journey of Paulie and Christopher in the "Pine Barrens" episode. This particular discrepancy is evidenced by those viewers who were left frustrated and dissatisfied with this ending. The series finale of Lost also polarised viewers. Many viewers, who thought the ending suggested that the characters were dead the whole time, were left frustrated and dissatisfied. But those viewers who understood that the characters were alive on the island and later reunited in the afterlife, were more satisfied (Outlaw).

It is difficult to say, but perhaps if Lindelof and his writing team had been allowed to write the story of Lost in fewer seasons, its ending would have provided more satisfaction and clarity for its viewers. The narrative trajectories might have provided a more direct, and therefore satisfying, path towards their intended ending-one with patterned randomness, rather than just apparent randomness. Regardless of whether this would have been the case or not, or whether the serial is deemed a success or not, it is clear that the ending of Lost reflected the nature of the show as a whole. Lost was a show that employed openended narratives, with episodic elements (such as framing each episode around a flashback to the life of a character before they crashed on the island). And as such, Lost applied the same balance to its series finale; providing answers to the questions posed throughout the narrative, but in a way that left many viewers confused.

What these examples show us is that where a serial drama decides to place itself on the spectrum of open and closed narratives, determines the way in which the entire narrative will, or at least should, unfold, but this does not mean that what happens in those narratives is predictable. Like a system that is being pulled towards a strange attractor, a successful TV serial drama utilises its self-contained and continuous narratives in a way that means its story unfolds in a way that is both unpredictable yet expected. In the following section I will examine the ways in which a successful serial drama is able to construct a narrative that is both expected and unpredictable through an analysis of The Leftovers. 


\section{Strange Attractors and The Leftovers}

The Leftovers begins its story three years after the occurrence of a global event, called "The Sudden Departure", in which $2 \%$ of the world's population vanishes without a trace. The serial follows the people left behind who are all struggling, in varying and conflicting ways, with the fact that they have to continue living their lives despite having no explanation as to where, why or how the departed disappeared. I have chosen to examine The Leftovers through the lens of strange attractors because the show's creator is Damon Lindelof and I think the serial serves as a remedy for the pitfalls of Lost that were mentioned above, as well as an augmentation of Lost's mastery. But mostly, I have chosen the show because it successfully employs distinctive self-contained narratives that contribute to, and are drawn into, the larger ongoing narratives of the serial as a whole. As a result, The Leftovers demonstrates the way in which a successful serial drama maintains its balance between being consistent but surprising, or unpredictable but expected, in the same way that a system that is being pulled towards a strange attractor demonstrates how a chaotic system displays disorder and order at once and is therefore deterministic but unpredictable. In order to demonstrate this point, I will examine how The Leftovers' treatment of its selfcontained narratives is the same at each scale-narrative threads within episodes, episodic narratives, seasonal narratives - and how this self-similarity is utilised in a way that enables the progress and escalation of the continuing narrative of the serial as a whole.

The Leftovers fits into the hybrid, or "flexi-narrative", model of TV drama narratives in that it employs open-ended narrative structures for the most part, but also provides a level of closure in its smaller narratives. In this sense, the narrative threads in The Leftovers can be compared to how the phase space curve of a strange attractor repeatedly forms loops and spirals, yet never intersects itself. An observer of such a system knows that the points of near-intersection will be pulled towards the attractor, but they cannot know the exact shape that these points will form (Gleick 150). We can see this similarity in The Leftovers' treatment of its narrative threads within its episodes, which are used to form the shape of the episode but also contribute, and are drawn to, the ongoing narrative of the serial as a whole. 
In the pilot episode, for example, we are introduced to each of the main characters in separate and disconnected narrative threads. Each of the Garvey family members, for instance, is introduced individually-in separates scenes, and occupying different spacesso that we don't know they are all a family until fairly late into the episode. This clearly establishes how fractured they are as a family and, in turn, that the Garvey family is functioning as a microcosm of society as whole, post-Sudden Departure. In this sense, the Sudden Departure is an inciting incident that sets the characters off on diverging paths, or trajectories. Yet, they are still connected by, and are drawn to, the same overarching trajectory that is driven by the lack of closure surrounding the Sudden Departure. In other words, the Sudden Departure has torn the characters apart within the world of the show, but the aftermath of the Sudden Departure is the thing that is pulling them together on a narrative level. This can be said for the serial as a whole, but it is particularly evident in the pilot episode which demonstrates how the individual narrative threads in a successful serial drama may appear to diverge wildly within an episode but are also pulled together. For example, following a scene in which Kevin Garvey tries and fails to convince the rest of the police force, and the mayor, that they need to be prepared for the potential danger of the Guilty Remnant (a cult that has formed in response to the Sudden Departure) turning up to the upcoming "Heroes Day" event, we cut to a scene in which Jill Garvey (Kevin's daughter, though we don't know it yet) is playing field hockey at school. In the hockey scene, we see two members of the Guilty Remnant watching the game from the sideline as Jill is shoved to the ground by another girl. In retaliation, Jill breaks the girl's nose with a violent elbow barge. While these two scenes work separately to progress the individual storylines of the characters, they also function together by foreshadowing what is to come (in the episode, as well as in the serial as a whole). That is, Kevin's prediction is correct-the Heroes Day event turns into a riot when the Guilty Remnant show up to perform a silent protest. But, as in the scene where Jill elbows the hockey player, the Guilty Remnant are not the ones to directly instigate the violence-it is the general public who become aggressive. They become aggressive because of the Guilty Remnant's insensitivity, but also because of their underlying frustration at not having any answers.

The violence at the Heroes Day event and in Jill's hockey game demonstrates that the characters in this world aren't going to be satisfied with an artificial sense of closure even if 
they try hard to appear to be satisfied. In broader narrative terms, from the placement of these two scenes, and other narrative threads in the episode and serial, a viewer can pick up on particular clues as to the general direction and shape of the narrative, without knowing what will actually happen. A viewer can realise that they are unlikely to get an answer about the Sudden Departure-because the drama is about lack of closure-but the serial manages to sustain a sense of unpredictability and surprise through its self-contained narratives which are used to depict the diverging paths of the characters as they struggle with this lack of closure. Like the behaviour of the phase space curve that is pulled towards a strange attractor, the self-contained narratives within The Leftovers are used in a way that contributes to the continuing and open-ended narrative of the serial as a whole.

We can also see this similarity between the episodic narratives in The Leftovers and the orderly and disorderly behaviour of the phase space curve of a strange attractor in the way that episodes in the serial will often appear to go full circle but are actually progressing in some way. An example of this is Kevin's storyline in the pilot episode. Kevin is introduced when he is going for a run and encounters a feral dog (see figure 4). When Kevin tries to tame the dog, a strange man appears and shoots the dog dead, claiming that "they're not our dogs anymore." At the end of the episode, Kevin encounters a pack of feral dogs and when the strange man turns up again to shoot them, Kevin joins in (see figure 5). It's essentially the same scene, but because of everything that has happened in the episode Kevin's attitude has changed-a marker of Kevin's personal growth within the episode, but also an indication of where the serial might be going. Kevin isn't just going to ignore the "feral dogs" any more, he's going to take action. In a broader sense, the chapter of Kevin trying to keep the peace in his town, and in his family, is closed, and a new chapter in which he's going to have to fight for peace is beginning. 


\section{This content is unavailable.}

\section{Please consult the print version for}

access.

Figure 4: At the beginning of the pilot episode, Kevin encounters a feral dog and tries to tame it. Screenshot by author taken from: "Pilot: The Leftovers, written by Damon Lindelof and Tom Perrotta, directed by Peter Berg, HBO, 2014.

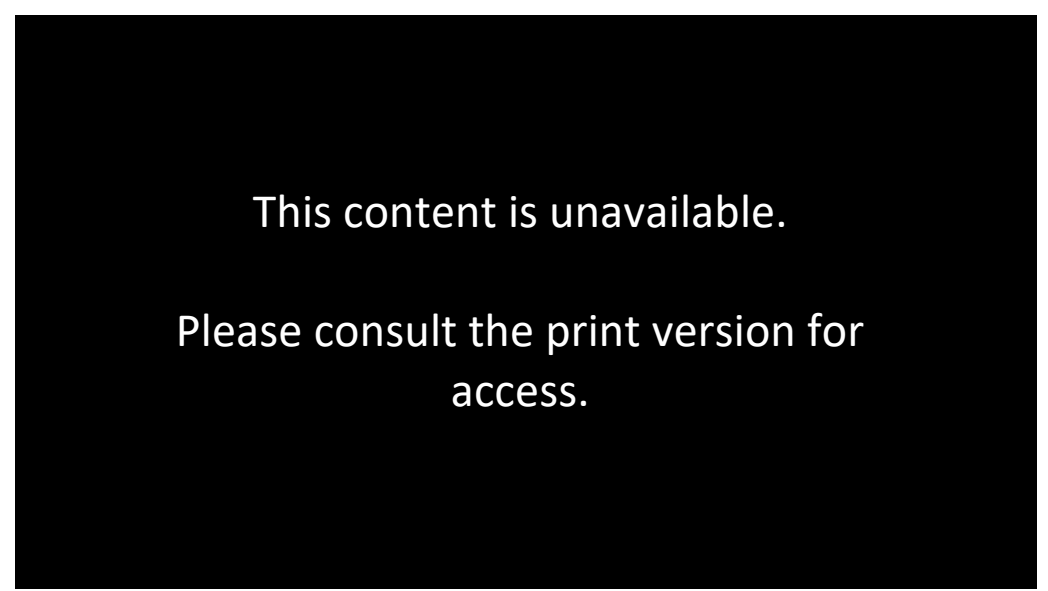

Figure 5: At the end of the pilot episode, Kevin encounters a pack of feral dogs and shoots them. Screenshot by author (ibid).

From this book-ending technique, we can see that, as well as containing smaller narrative threads within it, the episode itself has an overarching narrative shape, which establishes the show's degree of open-endedness that will be sustained throughout the entire serial. The overall shape of The Leftovers is also formed by the pulling together of distinctive episodes that often appear to diverge in an irregular way. While the second episode is largely a continuation or amplification of the pilot episode, the season, and serial, goes on to generate episodes with far more distinctive personalities, all of which nevertheless fit into the serial as whole in terms of tone and texture. The third episode, titled "Two Boats 
Helicopter", for example, focuses solely on Matt Jamison's perspective, in his desperate attempt to save his church from being bought out by the Guilty Remnant. The episode is compelling and, because of the positive reception from viewers and critics alike, the writers proceeded to produce more episodes with individual identities and self-contained narratives. Episode Nine of the season, titled "The Garveys at Their Best", goes back three years in time to depict where each of the members of the Garvey family were, and what they were doing, in the day leading up to the Sudden Departure. This episode not only sends the narrative in a new direction from the previous episode and raises new questions, but it also answers questions from previous episodes and therefore simultaneously provides a sense of closure. The episode gives the viewer a better understanding of why each of the characters have responded so differently to the Sudden Departure.

"The Garveys at Their Best" also comes after a dramatic episode in which Kevin is forced to witness the leader of the Guilty Remnant killing herself, ending with the distinct possibility that Kevin is going to be framed for her murder. This is a clear example of how the serial utilises the technique that Kristin Thompson describes as providing the viewer with the "appearance of design". By starting the next episode three years earlier, when none of the events we have seen so far have taken place yet, the writers defer the answers to narrative questions posed at the end of the previous episode in a way that gives the appearance of randomness-the narrative shifts in an unexpected and jarring way, whilst maintaining a sense of order and intent on the part of the writers. The further along we go in the season, and the serial, the more divergent and unexpected the episodic trajectories become, but this is always done in a way that is surprising rather than disappointing; unpredictable but expected. The gradual emergence of the show's treatment of its episodic narratives can be thought of in the same way that the return map demonstrates the innerworkings of a strange attractor. That is, the points of intersection on the return map may start off close together at the formation of a system but, after several iterations, eventually travel off in drastically different directions, before coming back together again. If The Leftovers had continued on the path that it was going down in the first two episodes, in which there wasn't much distinction between the shape of the episodes, the show might not have been as satisfying as it is. 
This delicate balance between open-endedness and closure, and between repetition and divergence, can also be seen in the shape of The Leftovers' seasonal narratives. The final episode of season one, titled "The Prodigal Son Returns", closely resembles the ending of the pilot episode but at a much larger scale. That is, where the pilot episode builds towards the danger of the Guilty Remnants' presence at the Heroes Day event, the season one finale builds towards the ramifications of the Guilty Remnants' act of placing doll replicas of all those who departed from Mapleton in the places where they disappeared. In doing so the first season of The Leftovers comes full circle, in the same way that the pilot episode does, but the two ends of the circle don't quite meet. There is not only a sense of expansion and intensification of the events that occur, but there is also a sense of progress in terms of where the characters end up. The next morning, after the fire and carnage has died down, there is a sense of catharsis as Kevin and Jill walk home, hand in hand, and are approached by the feral dog that they had taken in, which is now tame again due to Kevin's domestication of it (see figure 6). The season finale repeats Kevin's interaction with the dog that we saw in the first episode but, again, it is repeated in a new way to convey a sense of progress or change-offering a new sense of hope for the restoration of humanity, and for the characters going into the second season.

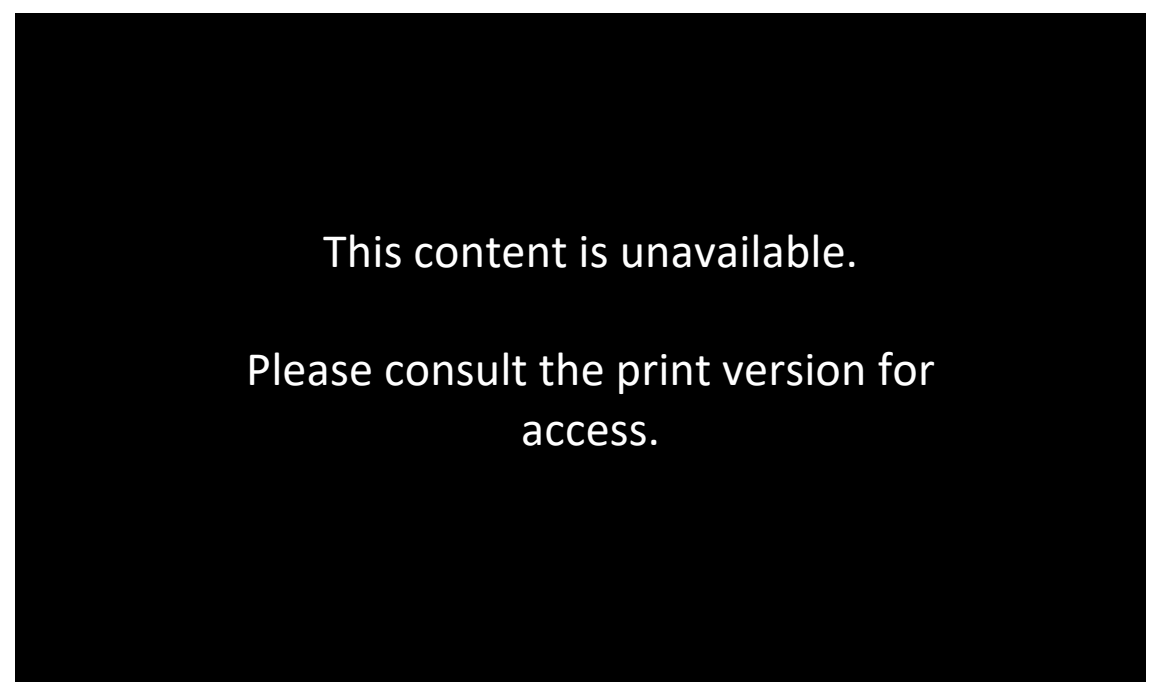

Figure 6: After surviving the carnage of the night before, Jill and Kevin are approached by a dog, once feral but now tamed. Screenshot by author taken from: "The Prodigal Son Returns": The Leftovers, written by Damon Lindelof and Tom Perrotta, directed by Mimi Leder, HBO, 2014. 
In the first episode of season two, titled "Axis Mundi", the writers take a huge risk in restarting the narrative in a completely new town, with a completely new set of characters-again demonstrating the show's diverging and unpredictable narrative trajectories. We are introduced to the Murphy family, who live in a town in Texas called Miracle-previously known as Jarden-the only place in the world where no one was taken in the Sudden Departure. This episode sets the season up in an opposite, or inverted, way from how the pilot episode set the first season up, yet by the episode's end the narrative is pulled back into a similar, but escalated, version of season one. Unlike the Garveys, the Murphy family is introduced as a close family unit from the outset. And Miracle, unlike Mapleton, is overflowing with hope and optimism. However, it is not long before this hope and optimism becomes cloying, and when the main set of characters from season one move to Miracle, the strange and mysterious events that season one dealt with set the characters off in similar-yet-different narrative trajectories (namely, that Kevin realises he is able to die, travel into a kind of afterlife, and return to tell the tale). Similarly, the third and final season of The Leftovers provides another shift in the direction of its narrative in that all the characters gradually end up in Australia. The season opens on Nora Durst as an older woman, living in rural Australia, and the season's arc functions as a catch-up narrative to answer the question of where Nora is exactly (is she in the real world? The future? The past?) and why she's there. And so, while each of the three seasons within The Leftovers shifts drastically, the narrative is still pulled towards the same ongoing narrative trajectory-bound up in the mystery and unknowability of the Sudden Departure-that was set up in season one.

Because the trajectory that the show's narratives are constantly being pulled into is shaped by the lack of closure surrounding the Sudden Departure, it sets up a huge challenge for the writers of the show to provide a satisfying resolution in its series finale. As I have mentioned, the show never promises to answer the question of what the Sudden Departure actually was, or why it happened, but this doesn't mean the show can end without some sense of closure for its viewers. Just as the season one finale was a kind of enlarged projection of the end of the pilot episode, the series finale of The Leftovers masterfully captures the sense of mystery that is at the heart of this season, but also of the show as a 
whole, while simultaneously offering answers to its narrative questions. The series finale, titled "The Book of Nora" ends with Nora (in Australia, in the future), telling her story to Kevin about how she travelled, via a machine built by scientists, to the place where the $2 \%$ are living. Since Nora's verbal account is all the viewer, and Kevin, have to go on, it is left open for interpretation as to whether she is telling the truth or not. Nora's story provides an ending for the serial that is simultaneously open-ended and closed. Viewers have two options in terms of what they can choose to believe (which, it should be noted, is "selfsimilar" to how the viewers have two ways of viewing Kevin's ability to come back from the dead; it's either real, or he's crazy). First, the viewers can believe Nora's story, and therefore have a sense of closure in the form of an explanation as to where the $2 \%$ of the population are, and so accept that Nora was able to see her departed family again. Alternatively, viewers can choose not to believe Nora's story, and instead find resolution in the fact that Kevin chooses to believe her story, and as a result find a sense of closure in the way that she and Kevin are content with their own manufactured closure. This ending embodies the serial as a whole, reflecting its treatment of open-yet-closed narratives at all scales. As I pointed out in my discussion of the first episode, the riot that breaks out at the Heroes Day Ceremony demonstrates that people aren't going to be satisfied with an artificial sense of closure even if they try hard to appear to be satisfied. The final scene between Nora and Kevin at end of the serial suggests that there is no other choice but artificial closure; the audience and the characters can choose to believe in a happy ending, or they can choose to not believe in the happy ending, but, either way, there's no way of knowing for sure.

By ending The Leftovers with such ambivalence, the serial adheres to the balance the show set up at the beginning and maintained throughout-between openness and closure, and between unpredictability and expectation. If the serial had given a definite answer, it would have tipped this balance, and derailed itself from its main trajectory. In this sense, like a system with a strange attractor which never settles down into a completely stable state, The Leftovers ends in a way that the narrative could potentially continue-questions are left unanswered, and some things are left unfinished. Because this open-endedness is present at all levels of the show's narratives-inter-episode narratives, episodic narratives, seasonal narratives-The Leftovers is able to generate a sense of closure from its open-endedness. 


\section{Conclusion}

The mixture of closed and open-ended narrative structure that successful serial dramas, such as The Leftovers, employ can be compared to the simultaneous order and disorder that is displayed in a system being pulled towards a strange attractor. As Gleick explains, the Hénon attractor can be viewed through progressive magnification to reveal the unfolding of its regressive lines within lines, or it can be viewed in reverse so that an eerie shape emerges out of its points over time. The same can be said for the ways in which we can view any successful serial drama. We can view the serial as a whole and go backwards to examine the regressive nature of its self-similar narrative structures. Or we can view the serial as it unfolds and see the diverging narrative structures come together to form the shape of the serial as a whole. Together these two views demonstrate a show's patterned randomnessin one direction the serial appears to unfold erratically, in the other direction we can clearly see the pattern and repetition underlying the serial.

In the previous chapter I concluded that "Universality" is a useful tool for someone who is new to writing or analysing TV serials to gain a better understanding of the simple structures and rules that underpin them. In a similar way, strange attractors are a useful tool with which to better understand how these simple structures and rules are used to produce expected yet unpredictable storylines. Just as strange attractors explain the behaviour of dynamical systems that display order and disorder at once and that never stop or settle down but also never repeat or intersect, strange attractors can be useful for a TV analyst or new TV writer to better understand how successful serial drama narratives repeat and progress at the same time, are comprised of endings that are also beginnings, and are contained but ongoing. That is, strange attractors can be used as an abstract and visual representation for how unpredictability and surprise are simultaneously built into and out of the familiar and repeated patterns that are inherent in all successful serial drama narratives. In this sense the process of writing, and watching, a successful TV serial is similar to the stretching and folding of phase space, "in the manner of a pastry chef who rolls the dough, folds it, rolls it out again, folds it, creating a structure that will eventually be a sheaf of thin layers" (Gleick 149). 
I am now going to turn to my creative component which comprises three separate TV projects that have both informed and been informed by my examination of the similarities between chaos and serial drama narratives. Each script will be accompanied by a brief overview of my experience of writing each serial, touching on the discoveries and surprises that shaped each one and demonstrating the way in which the TV writing process is like that of a pastry chef rolling dough into a sheaf of layers. 


\section{PART TWO: \\ CREATIVE COMPONENT}





\section{INTRODUCTION}

When I began my PhD, I expected that the organisation of my critical component would be clear and simple. I thought I could discuss different "branches" of chaos and apply them to the different aspects of serial drama narratives. I thought I could discuss fractals, "Universality", strange attractors, and sensitivity to initial conditions in isolation to each other, when in reality all chaotic systems possess characteristics of each of these aspects of chaos. This made it difficult to categorically align a particular component of serial drama narratives with a particular aspect of chaos without worrying that I was not addressing the fact that that particular component of serial drama narratives could also be aligned with another aspect of chaos. A further challenge was that serial dramas aren't chaotic systems - I was simply attempting to address the similarities between the two, and particular branches of chaos were never going to perfectly map onto different aspects of this narrative form. I eventually came to realise that the difficulty of isolating particular characteristics of chaos highlighted the very complex nature of serial drama narratives that I wanted to demonstrate. That is, serial drama is a narrative form in which all aspects of its story are connected and should be deemed a complete entity, while simultaneously possessing various parts that can be named and identified as separate components, even if they can't be solely isolated from other components.

When it came to my creative component, the problem of overlapping areas of chaos also presented itself. Again, I had proposed to write three TV serials that would each implement an individual branch of chaos. But because it was difficult to separate the aspects of chaos, each script ended up combining all of them to varying degrees. And so, as with my critical component, rather than applying a single branch of chaos to a particular narrative technique in each serial, I tried to use a dominant aspect of chaos in each serial whilst incorporating other aspects. Untold is a serial that was inspired by fractal geometry, but also has elements of strange attractors and sensitive dependence; Bad News was modelled around the idea of strange attractors, but also has characteristics of fractals and sensitive dependence; The 
Chances has sensitive dependence at its core, but also incorporates characteristics that are similar to fractals and strange attractors.

And each of these serials uses the conventions and formulae of TV drama narratives that I discuss in relation to "Universality". This means that, while the narrative structures and formulae in most serial dramas remain invisible to a viewer, the serials that I have written use these underlying structures to generate narratives that are chaotic in both form and content. The following TV serials are chaos-like on both a structural and story level.

I will be accompanying each individual serial with an outline of my experience of the writing process, which will provide more detail about how each serial implements the ideas of chaos at the levels of both structure and story. These outlines will also demonstrate how my experience of writing each serial with chaos in mind allowed me, as a new writer, to navigate the process of writing for TV, which I often find to be an overwhelming and convoluted process. That is, writing a serial drama is a challenging undertaking due to the vast number of interweaving storylines and characters that has to be produced in a coherent and engaging way. A specific aspect of this challenge is having to consider the short-term storylines (a character does something in an episode, for instance), while also thinking about the long-term storyline (how a character's action in an episode will impact, and fit into, the story later in the season or serial). While chaos doesn't provide a solution to the problems that stem from the sprawling nature of serial drama narratives, I will be demonstrating how chaos has been a useful tool for understanding why some aspects of the writing process have been difficult, while other aspects of the writing process came more easily to me. The first serial I am presenting is Untold. 
UNTOLD

Written by

Alison McLachlan 



\section{UNTOLD}

\section{$6 \times 1$-hour drama series}

When Letha Maven, a kindly Grandma, is sent to prison for murdering her two babies fifty years ago she quickly proves there is far more to her than her frail exterior suggests.

In an attempt to manipulate her fellow inmates into thinking she's harmless and innocent, Letha tells them a series of embedded stories.

But when Sara, the tenacious main character in Letha's story, breaks into Letha's consciousness, Letha struggles to suppress her cold-blooded tendencies that have been lying dormant for decades.

In an attempt to stand up against Letha and break out of her mind, Sara clashes with the off-kilter projections of Letha's family and acquaintances by unleashing Letha's dreams, memories, traumas and dark family secrets.

As Letha's calm façade slowly unravels and she comes to embrace her malicious side, and as Sara struggles to gain control of her own life in the face of a crushing existential crisis, the two are caught in a battle against each other in which there can only be one victor.

Will Letha escape her sinister past and defeat Sara? Or will Sara gain free will and reveal the disturbing truth about who Letha really is? 

CHARACTERS 


\section{LETHA}

77.

Letha Maven is callous, selfish, and narcissistic, but she's also charming, confident and a masterful manipulator.

Letha has been aware her whole life that she doesn't feel and experience things like other people do, but over the years she's perfected the art of deception-cunningly convincing everyone around her that she's sensitive, empathetic, and harmless. She loves the thrill she gets from tricking them.

Letha's biggest and, in her eyes, most successful deception is that she is a doting and nurturing mother and grandmother even though she's never felt an ounce of love or compassion for her family. She's merely using her family to sustain her kindly façade, concealing the dark and sinister tendencies that fester beneath.

Because she's always felt in control, when Letha doesn't get what she wants, or someone sees through her act, she is very quick to snap.

So, when she is sent to prison for murdering two of her own babies fifty years earlier and the whole world starts to see her for who she really is, her tightly wound persona gradually unravels until she can no longer contain her maliciousness.

\section{SARA}

19.

Sara is strong-willed, tenacious, and rebellious, but she is also naïve, fool-hardy, and selfinvolved.

When Sara breaks out of her story world-where she is accused of causing plague in a small medieval town-she is thrown into a crushing existential crisis and immediately sets out to seek revenge on Letha for causing it.

But, more than anything, Sara wants to be free and she will stop at nothing in order to achieve it.

Sara's desire to be free often blinds her. Her ruthless determination to be in control often leads her into onerous situations in which she loses the very agency she is seeking.

As much as she disdains Letha, Sara comes to realise she has a lot more in common with her than she'd like to admit. In order to avoid becoming Letha, Sara must learn how to consider other people's feelings and perspectives if she's ever truly going to be free. 


\section{SUPPORTING CHARACTERS:}

JULIAN (70): Julian is Letha's ex-husband, and the Malcolm's estranged father. Julian is kind, compassionate, conscientious and selfless but he is weighed down by deep sadness and grief. More than anything else, Julian wants to reunite with his family who ostracised him after he dobbed Letha into the police for killing his and Letha's babies.

While he is part of the family in Letha's consciousness, everyone there has been convinced by Letha that Julian is the one who killed his and Letha's baby daughters. The Julian in Letha's mind and the Julian in reality must both learn how to find it within themselves to forgive and forget Letha in order for the both Julians to take control of their lives and be accepted by their family.

MALCOLM (43): Malcom is Letha's doting son. He is self-righteous and stubborn, and while this often gets him ahead in life, it also makes him cold and detached - not that he realises it. He is dedicated to proving his mother's innocence and getting her out of jail. But, blinded by his love for her, he's failed to see that she's actually a psychopath and has never loved him in return. So, when Malcolm learns the truth about his mother, he must overcome the overwhelming consternation and bitterness he feels in order to keep his family together.

In Letha's consciousness, Malcolm's self-righteous and stubborn qualities are heightened so that he is at his mother's every beck and call. He must cut his apron strings if he's ever to gain independence from Letha.

KIM (22): Kim is the Letha's energetic, talkative, and friendly cellmate who is quick to welcome Letha into prison. But when Kim learns that Letha murdered her babies, she enters into a feud with Letha that brings out the dark and vitriolic side of Kim's personality which she's been working hard to keep under control. Kim has to fight her insatiable urge to make Letha pay for what she did if she is to ever to return home to her own baby.

In Letha's consciousness, Kim's vindictiveness and disdain of Letha is exacerbated to such a degree that she makes Letha look like a nice person in comparison.

MEGAN (56): Megan is one of Letha's fellow "mature" inmates. She's a hard case, but loyal and caring to those who earn her respect and trust. As a result, people know not to mess with Megan. But when Letha arrives refusing to express remorse for her crime, Megan's level-headedness is put to the test.

Despite their strained and tense relationship in real life, Megan is gushing, warm and affectionate in Letha's consciousness. 
CHIEKO (38): Chieko is Letha's daughter-in-law. She is a strict but loving and caring mother to her two daughters, Letha's grandchildren. She's had to grow a thick skin in order to deal with Letha's constant cruelty towards her, which Chieko interprets as jealousy - not that she'd ever tell Malcolm. When the truth comes out about Letha, Chieko has to admit to Malcolm that she's always secretly known that Letha killed her babies, putting their marriage in jeopardy.

In Letha's consciousness Letha has separated Chieko from her family and forced her to be the main servant of the house. So, when Chieko hears about Sara's intention to rebel against Letha, Chieko is one of the few people who join her.

ROSE \& PHILIP MAVEN (50s): Rose and Philip are Letha's parents who we only ever see from Letha's perspective in her consciousness. They are both very overbearing, regimented, and controlling, which stems from their role in forcing Letha to give her first baby away when she was a teenager. If there is ever a problem in Letha's consciousness, their solution is to execute whoever they think is responsible - even if there's no evidence to prove it.

THOMAS (13): Thomas is Sara's saviour in Letha's story. He is sensitive and empathetic but he's hardwired to pretend that he isn't. Thomas is convinced that Sara is going to change his tumultuous life for the better (much like Julian when he first met Letha), but he must come to the difficult realisation that he's been manipulated, and must figure out how to live out the rest of his life on his own, knowing it's all made up.

GRANDPA MAVEN (65): Grandpa Maven is cruel and villainous and the one person that can directly cause Letha pain. After being trapped for decades, Grandpa Maven breaks out of a memory in which he molests Letha, causing havoc in Letha's consciousness.

EMILY \& CHARLOTTE (7 \& 9): Emily and Charlotte are Letha's two granddaughters. They adore Letha more than their own parents, so when she is sent to prison their lives are completely rocked.

WILLOW (40s): Willow is Letha's mysterious and enigmatic cellmate who refuses to speak. 


\section{UNTOLD}

Episode Synopses (1-6) 



\section{UNTOLD}

\section{Episode One: "Scapegoat"}

After spending the night with her two granddaughters, telling them a heart-felt bedtime story, LETHA's (70s) life is turned upside down when she is sent to prison for the murders of her two babies half a century ago.

While Letha is welcomed by her cellmates - KIM (20s) and WILLOW (30s) - she grows irritated with MEGAN's (60s) obvious contempt for her and her crime, which Letha insists is a misunderstanding.

When the prison's TV breaks down in the middle of a soap opera, Letha attempts to win the angry and reluctant inmates over with a story about Sara, a young, pregnant woman who is captured in a medieval town after she is accused of being a witch and causing a plague.

While Letha comes to blows with Megan after she makes another dig about Letha's lack of remorse for what she did, SARA (20s) comes to life in Letha's consciousness-a mansion occupied by off-kilter versions of Letha's acquaintances, and ruled by alternate versions of Letha and her family.

That night, once Letha finally manages to get to sleep in her prison cell, Sara travels into Letha's dreams where she is comforted by a friendly anthropomorphic tree after escaping an underground labyrinth and murdering Letha's two granddaughters.

After being told not to bring anything back from Letha's dreams, Sara-curious to see what will happen when she does - sneaks the seed of the anthropomorphic tree into Letha's consciousness.

In the morning, Letha's consciousness is in a state of disarray as Sara's seed has grown into an enormous, destructive tree that continues to expand uncontrollably. Wanting to send a message to anyone who is thinking of breaking the rules, Letha's family execute a young boy after he is framed by Letha's ex-husband, JULIAN (70s), for bringing in the seed.

After Letha chillingly confesses to Megan that she doesn't feel guilty for killing her babies, and never has, Letha resumes her veneer of innocence when she receives a call from her son, MALCOLM, who claims he can prove that Letha is not guilty.

Convinced that Letha is an evil person, Sara sets out to rise up against her when she is approached by Julian who desperately requests her help in destroying one of Letha's memories. When Sara learns that doing so could harm Letha, she enthusiastically agrees. 


\section{UNTOLD}

\section{Episode Two: "Groundwork"}

While Letha struggles to contain her animosity for Kim after she continuously refuses to listen to Letha's assertions of innocence, Sara sets off on a dangerous journey to retrieve a memory of Letha's for Julian.

Sara encounters an escaped apparition, GRANDPA MAVEN, on her journey and, thinking he is also trying to sabotage Letha, she makes a deal with him - he'll tell her everything he knows about how to navigate long-term memories, if she promises to make Letha remember him.

Meanwhile, Letha's frustration with Kim's defiance finally mounts, and she makes a deal with a group of hardened criminals - they'll hurt Kim for Letha, if Letha smuggles contraband into the prison.

When Sara draws the unwanted attention of security in Letha's long-term memories, Sara becomes trapped in a memory in which Julian kills one of his and Letha's babies. There she meets JOAN who is also trapped, and claims to be one of many story characters tricked by Julian, and forgotten by Letha.

After Letha collects the contraband she is horrified to learn that she's locked out of the prison but, while trying to find a way back in, her spirits are lifted when she witnesses Kim being beaten to a pulp.

As Sara's desperate attempt to escape the memory she's trapped in fails, Letha delivers the contraband before forcing her fellow inmates to listen to the next instalment of her story:

In attempt to defer her execution, Sara tricks Thomas into listening to her story about a boy similar to Thomas who kills his abusive father (who resembles Grandpa Maven) after being told to do so by some underground dwellers, only to find that they have never existed.

Sara has to cut the story short because the medieval guards arrive to take her to be hanged, and Letha has to cut her story short because she realises she still has some remaining contraband on her person.

Sara is relieved when she finds herself back in Letha's consciousness, but as she and Julian burn, what Julian insists is, the false memory of him killing the baby, Sara is enraged to learn that she never escaped the memory - Letha merely duplicated Sara in order to tell her story.

More determined than ever to get back at Letha for her cruelty, Sara plays Letha's memory of Grandpa Maven in which he molests Letha as a teenager, triggering Sara's tree to grow again until it envelops the entire mansion and causes complete destruction.

Meanwhile, a guard discovers Letha in a catatonic state after she has tried to return the contraband. 


\section{UNTOLD \\ Episode Three: "Smoke Screen"}

After her apparent psychotic episode, Letha is taken into the prison's hospital wing where she sleeps, heavily sedated, next to the brutally injured Kim.

When Sara finds herself trapped in an eerie world where she observes a 19-year-old Letha on the verge of suffocating a new-born baby, Sara tries to interfere until she sees that Letha is actually trying to stop her parents from giving the baby up for adoption.

While Malcolm tries to use Letha's mental issues to get her out of prison, Sara attempts to find a way to escape Letha's memory in which she witnesses Letha's parents ordering her to keep the secret about Grandpa Maven being the father of her baby.

Meanwhile, still trapped in Letha's memory, Sara witnesses Letha's mother hiring a psychic who forces Letha to lock the memory of the whole experience away in her mind.

After Sara-feeling sympathy for Letha for the first time-forces Grandpa Maven to apologise to Letha, the memory fades and Sara finds herself back in Letha's consciousness where she learns she has been knocked out and captured by Letha's family who have no knowledge of Letha's memory of Grandma Maven and the baby.

When Letha comes out of her sedation, Letha manipulates Kim-unaware that Letha was responsible for her injuries-into apologising to her. In return Letha tells Kim the next instalment of her story:

Thomas saves Sara from execution after he stabs the executioner and his own father (aka Grandpa Maven) and, while on the run together, Sara continues her story to Thomas:

The young boy who resembles Thomas is preparing to bury his father's body when a stranger appears, requesting shelter for the night. In order to distract him from the corpse, Thomas reluctantly listens to the stranger's story:

A man who gets cold feet on his wedding day skips town and makes a new life for himself but, years later, his ex-fiancée tracks him down and tricks him into thinking they've been married that whole time and his old life was just a dream.

The story stops when a wild animal discovers the corpse of the boy's father, and when Sara is paralysed by the traumatic memory of Grandpa Maven. In order to escape again, Sara offloads the memory onto Thomas who is steadily becoming conscious of his existence as a story character.

While Sara overhears Letha's family making a plan to abandon Letha's consciousness and travel to a utopia in order to escape the potential danger that Sara's abilities pose, Letha tells Malcolm about the baby she had to give up for adoption. But when Malcolm looks into it, he is horrified to learn that she has made Grandpa Maven, and the whole experience, up to gain sympathy from others. 


\section{UNTOLD \\ Episode Four: "Life"}

After Letha undergoes an intensive psych test that Malcolm hopes will mean she can come home, Letha is approached by the hardened criminals who order her to smuggle more contraband into the prison as repayment for the items she lost.

Meanwhile, when Sara and Chieko join forces to rally everyone in Letha's consciousness to rise up against Letha's family, a riot breaks out until Letha's family surrenders and promises to take everyone to the utopia.

Letha is thrilled to be accepted into the group of hardened criminals after she impresses them with her cunning ability to deceive, but her joy is upturned when she learns that the test results have deemed Letha to be a psychopath and her ruse is finally up.

After hearing that Malcolm is planning on sending her to a psych ward, rather than bring her home, Letha tries to regain her sense of control by telling a new instalment of her story:

Sara and Thomas are still on the run when Thomas catches the plague and Sara takes them to a peculiar place where social outcasts go to live in peace. Here, Sara tells Thomas a new instalment of her story:

The young boy who resembles Thomas watches his dad's corpse being mauled by the wild animal while listening to his guest continue his story:

After memories of the runaway fiancé's old life come back to him and his fake wife convinces him that he is going crazy, he decides to end the immense confusion by killing himself but is interrupted by Death who tells him the story of his origin:

The world was once boundless-everything was nothing, and nothing was everything-until a single black streak appeared, which meant that nothing now had meaning because it could be compared to everything. Death posed a question to the man that would help him in his decision to live or die; was the man everything, or was he nothing?

The young boy cuts the man's and Sara's story short when he realises that his the father in the story is Grandpa Maven from Letha's memory. Alarmed that Thomas is gaining awareness of his existence, she takes him back into Letha's consciousness where she discovers that everyone has left for the utopia without her.

While Letha orders the hardened criminals to burn her son's house, Sara is met by a panicstricken Julian who tells her that his family are actually taking everyone to an inescapable and torturous part of Letha's dreams. Rather than saving them, Sara flees to the lighthouse which she believes is her only hope of escaping.

In the lighthouse, after Sara observes a recording of Willow - Letha's mute cellmate-telling an emotionless Letha that her granddaughter was killed in a house fire, Willow pulls Sara into the real world. 


\section{UNTOLD}

\section{Episode Five: "Freedom"}

When Letha is taken to the psych ward and put in an isolation unit, the silence and loneliness drive her completely insane, and in order to distract her from a waking nightmare, she tears her clothes off and makes a rope out of them.

Meanwhile Sara navigates the real world-feeling thrilled and powerful-but when she goes into labour she realises how alone and miserable she really is.

In Letha's mind, Letha's family set out to find their utopia after trapping everyone else from Letha's consciousness in the waking nightmare that Letha keeps experiencing.

After Sara gives birth to her baby in a hospital, the nurses and doctors become alarmed when they find no proof of Sara's identity or existence, forcing Sara to go the run with her baby.

Sara is driven to breaking point after struggling to care for her baby alone and, in order to put an end to the torment, she kills the baby and realises she is exactly like Letha.

Not quite willing to accept her similarity to Letha, Sara does what she thinks Letha would never do-she tracks down Letha's family to make amends. But she is surprised to find Charlotte-Letha's granddaughter who was allegedly killed-celebrating with the rest of the family over a lovely meal.

Letha's family are dismayed to discover Sara in their presence, and are about to capture her when they receive a call from Letha's psych ward saying that Letha is in a coma after an attempt to hang herself and that she left a note asking for Sara to visit her.

While Sara and Letha's family visit the unconscious Letha, Sara is alarmed when she is the only one who is able to see that everyone from Letha's consciousness is in the room with them, suffering in silent agony. Sara comes to the horrible realisation that she is still in Letha's head, and that she has made it to the supposed "utopia", a replica of Letha's reality.

When Sara, enraged, attempts to murder Letha once and for all, Letha comes out of her coma and reveals that Sara's experience in Letha's consciousness is all just a story as well, and Letha pleads for Sara to help her in tracking down Willow who, Letha claims, is their creator. 


\section{UNTOLD}

\section{Episode Six: "Ends"}

After Letha and Sara bond over their plan to seek revenge against Willow, Letha slips back into her coma and is put on life support, forcing Letha's family to make a decision as to whether to keep Letha alive or let her die.

Sara sets out on her own to find Willow but soon realises she's stuck in a loop-doomed to repeat the same actions over and over again-and the only chance she has at stopping it is to finish Letha's stories.

After Letha's family decide to turn Letha's life support off, Sara intercepts and tells Letha how the rest of her stories end:

After the man's visit from Death, he decides not to kill himself and instead goes looking for his old family as proof that he isn't crazy but, before he can, his fake wife knocks him over the head. The man wakes up with no memory at all of his old family, and lives out the rest of his life with his fake wife. The story-world disintegrates into sand.

The man telling the young boy his story discovers the boy's father's mauled corpse, so the boy kills the man too, buries both their bodies in the hole that his father made him dig, and discovers that his life is much more peaceful without his father and that he no longer regrets what he did. The story-world turns into sand.

Upon finishing her story, Sara's pregnant belly has vanished and Sara reveals to Thomas that she really is a witch and is the one who caused the plague, and that she has been manipulating Thomas' actions this whole time.

Thomas tries to convince Sara to stop her witchcraft, but she insists it's what she was born to do. She tells Thomas that she can cure him of the plague if he wants but he'll have to live a life on the run with her. Thomas chooses to die instead. Sara leaves him and continues to spread sickness and death throughout the world. The story-world turns into sand.

Sara returns to Letha's real consciousness, where everyone but Letha's family is now preparing for an impending apocalypse.

After finally accepting that there is nothing more she can do to alter her fate, Sara is given the power to decide how to end the story that Letha was telling her granddaughters before she was imprisoned. In order to provide a sense of peace and harmony for everyone, Sara ends the story on a happy note.

When Letha's family turn Letha's life support off and Letha slowly dies, Letha's consciousness, and everyone else in the "real world", disintegrate.

We travel out to reveal that Willow, a psychic, is telling this story in the distant past to Letha's mother to show her what will happen to Letha, a toddler, if she doesn't do something about the extremely disturbing behaviour that Letha's been exhibiting. 


\section{UNTOLD}

Scripts (Episode One \& Episode Two) 

UNTOLD

Episode One:

"Scapegoat"

Written by

Alison McLachlan 

EXT. MANSION - DAY

A clump of sand that mottles an otherwise green, manicured lawn is picked up by a strong gust of wind.

The sand whirls towards a grand mansion that looks like a life-sized dolls house - except for the weatherboard that is missing from beneath the front window.

The sand lashes against the side of the mansion and, as if by magic, mutates into the missing weatherboard.

INT. MANSION, SITTING ROOM - DAY

Despite the strong winds outside, FINLEY (7) joyfully plays with her cat. She drags a piece of string across the floor and giggles as her cat chases after it.

She scoops her cat up and cuddles it lovingly, before sinking into a comfy armchair.

Her cat purrs appreciatively on her lap as she strokes him.

They both grow sleepy

Finley closes her eyes, and drifts off to sleep.

While she's sleeping, a gust of wind blows the front door open. The cat skittishly launches off Finley's lap and scurries out the open door.

INT. MANSION, SITTING ROOM - DAY (A FEW HOURS LATER)

The wind has died down, but the door is still wide open.

Finley is still asleep on the arm chair, when her MOTHER angrily storms in, and stomps her foot on the ground.

Finley wakes with a jolt.

MOTHER

I told you to keep the door closed.

Finley looks at the open door, groggy and confused.

I did.

FINLEY

The Mother puts her hands on her hips in cartoonish anger. MOTHER

Clearly you didn't. And now the cat's escaped.

Finley surveys the room for her cat. She shakes her head in disbelief. 


\title{
FINLEY
}

It must have been the wind!

\author{
MOTHER \\ Stop lying, and go to your room at \\ once!
}

\section{FINLEY}

But Mother, you have to believe me!

It wasn't my fault!

The Mother isn't going to budge.

Finley, tears in her eyes, bows her head and trudges out of the room.

INT. MANSION, BEDROOM - DAY

Finley opens her window shutter, and makes an escape out the window.

EXT. MANSION - DAY

Finley desperately scours the front lawn.

FINLEY

Here kitty kitty.

Finley listens for a response, but all is quiet.

Muffled voices penetrate the silence, but Finley doesn't notice.

$$
\begin{aligned}
& \text { CHIEKO (O.S.) } \\
& \text { Time to sleep, girls. } \\
& \text { EMILY (O.S.) } \\
& \text { Noooo! }
\end{aligned}
$$

Finley's eyes roll back in their sockets. Her knees go weak, and she collapses to the ground.

$$
\text { I want to hear how it ends. }
$$

As Finley remains sprawled on the ground, the world around her becomes pixelated.

Finley wakes up again - her eyes have returned to normal, but she now looks utterly confused as to where she is.

She gawks at the pixelated mansion as it disintegrates into a pile of sand right before her eyes. 
Finley darts her head around in panic as the entire world around her disintegrates into sand, and she finds herself in the middle of a vast desert.

The wind blows strange patterns in the sand.

Finley sees a faceless figure scurry across a sand dune, running freely.

$$
\text { Help! FINLEY }
$$

The figure runs so fast that he turns back into sand.

From the sand another faceless figure appears. At first he walks and then he jogs, then sprints until he too turns into sand.

Finley sees a wave of sand heading towards her.

She lets out an ear-splitting scream as she runs then sprints then disintegrates into sand.

TITLES.

INT. SHITTY MOTEL ROOM, BEDROOM - NIGHT

LETHA MAVEN (77) is sitting in a motel bed with her two pyjama-clad grandchildren - CHARLOTTE (9) and EMILY (7) cuddled up next to her.

The girls' mother - CHIEKO (38) - has her arms crossed against her chest, and eyes her daughters impatiently from the doorway.

$$
\text { Pleeease! CHARLOTTE/ EMILY }
$$

Chieko shakes her head, sternly.

$$
\text { CHIEKO }
$$

You can hear how it ends tomorrow night.

Letha flinches at Chieko's comment, and glares at her. Chieko glares back, until Letha finally relents.

LETHA

Mothers know best, girls.

Charlotte and Emily grizzle and moan as Letha pulls them in for affectionate hugs and kisses. 
The mother in that story didn't know best.

Letha laughs at Charlotte's astuteness as she extracts herself from the bed.

She stands over the girls with a hint of sadness in her eyes. LETHA

Sweet dreams, chickadees.

Night, Grandma.

EMILY/ CHARLOTTE

Letha bows her head and trudges past Chieko, rudely bumping into her shoulder as she does so.

INT. SHITTY MOTEL ROOM, LIVING AREA - NIGHT

MALCOLM (43 - has a distinct lazy eye), is sitting on the couch reading over some files.

Letha stands behind him.

LETHA

I really think you ought to warn them.

Malcolm closes his eyes, to calm himself.

$$
\begin{aligned}
& \text { MALCOLM } \\
& \text { Warn them of what, Ma? }
\end{aligned}
$$

Letha sighs - she can't be bothered.

$$
\begin{gathered}
\text { LETHA } \\
\text { Goodnight, Malcolm. }
\end{gathered}
$$

Letha leans over her son and kisses him on the top of his head.

Malcolm watches Letha hobble out of the motel room.

EXT. MOTEL PATH - NIGHT

Letha continues down the outdoor path that leads to a neighbouring motel room.

As she fumbles to unlock her door, she glances at a police car that is parked in the car park outside her room. 
INT. SHITTY MOTEL ROOM 2 - NIGHT

Letha flicks the light on. Her room is smaller and seems colder.

Letha undresses, puts on a nightgown and slips into bed.

She flicks the light off, anxiously stares up at the ceiling, and listens to the murmur of her family chatting and giggling next door.

INT. SHITTY MOTEL ROOM 2 - MORNING

Letha jolts awake to an urgent knock on the door.

Ma!

MALCOLM (O.S.)

Letha hobbles to the door as Malcolm continues to knock.

Letha opens the door to a flustered-looking Malcolm.

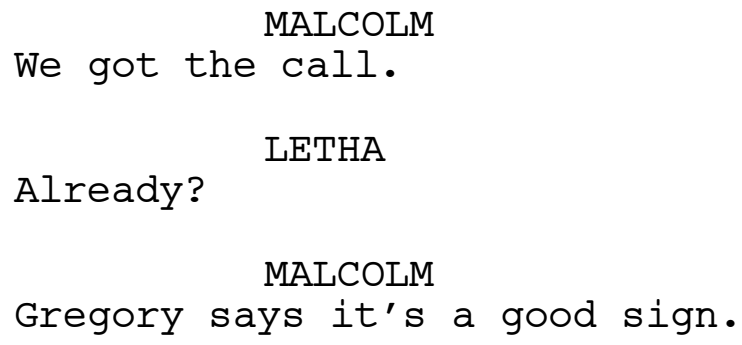

Letha nods, hopeful but doubtful.

LETHA

The girls?

MALCOLM
Chieko's dropping them off at
school early.

Letha drops her gaze, sadly. She trudges back into her room.

Malcolm closes the door, and Letha gets dressed into a

"formal" outfit as quickly as she can.

INT. COURT ROOM - DAY

The room is humming with apprehension.

Chieko and Malcolm are sitting in the public seating area with several other ANXIOUS PEOPLE, including JULIAN (75 friendly and approachable).

Letha is sitting with her lawyer, GREGORY (50s - gruff and worn-out), at the defence table. She is staring at her clenched hands. 
Everyone else's eyes are on the JUDGE.

$$
\begin{aligned}
& \text { JUDGE } \\
& \text { Will the foreperson please stand? }
\end{aligned}
$$

In the jury box, the FOREPERSON - an elderly woman about the same age as LETHA - stands up, holding a form.

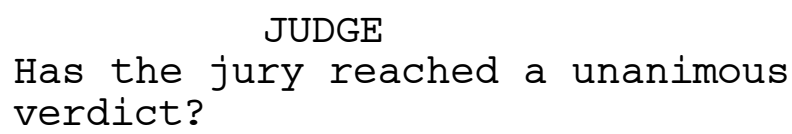

The court attendant walks over to the foreperson, takes the form from her and hands it to the judge.

The judge reads over it silently.

Letha tries to steady her trembling hands.

$$
\text { JUDGE }
$$

The court attendant will now read the verdict.

The court attendant takes the form, and begins to read it out.

\section{COURT ATTENDANT}

"We the twelve members of the jury

unanimously find the defendant to be guilty on two counts of first degree murder."

Letha's face twitches ever so slightly, but she still doesn't shift her gaze.

Gregory is gobsmacked.

Malcolm lets out a guttural sob that echoes throughout the court room.

Julian closes his eyes, sadly relieved.

COURT ATTENDANT

On this fifth day of September, two

thousand eighteen....

The court attendant's voice is subsumed by an ascending ringing in Letha's ear. She still doesn't move.

Two POLICE OFFICERS pull her up and cuff her.

$$
\begin{gathered}
\text { GREGORY } \\
\text { This isn't the end. }
\end{gathered}
$$

The police officers usher her out of the courtroom. 
Letha doesn't look back.

INT. PRISON PROCESSING ROOM - DAY

A clock on the wall ticks excruciatingly loud.

Letha - calm and still - is sitting at a table in a sterile room, handcuffed. It looks like she's been here a while.

There is an empty seat across from her. Files about Letha are spread out on the table in front of the empty seat, as well as a glass of water.

Something in Letha snaps.

She lunges for the glass of water, and hurls it towards the clock. She misses terribly due to her handcuffs and frail arms.

The water spills over the table, and the glass smashes on the floor.

Letha takes her seat again, returning to her calm and still position like it never happened.

The clock continues to tick.

A CORRECTIONS OFFICER enters the room. When she gathers the files, she notices how wet they are. She glances around the room and spots the broken glass on the floor.

She glances at Letha, questioning.

Letha amps up her frail and grandmotherly demeanour.

LETHA

I'm so sorry, dear. I was parched, so I tried to take a sip of your water.

The corrections officer looks confused, then sees how pitiful and frail Letha looks.

The corrections officer waves the files to rid them of the remaining droplets of water.

No worries.

CORRECTIONS OFFICER

INT. CUBICLE - DAY

The corrections officer hands Letha over to a GUARD before leaving. 


\section{GUARD}

Take off all garments and place

them in the container to your left.

Letha looks horrified.

$$
\text { May I have some privacy? }
$$

The guard shakes her head.

GUARD

Better get used to it, hun.

Letha tentatively takes off her clothes, she folds them up carefully before placing them into the container.

She shivers in the middle of the cubicle, her weathered body laid bare.

The guard proceeds to inspect her - in every nook and cranny.

INT. PRISON - DAY

Letha, now wearing a beige jumpsuit and carrying a small bundle of prison essentials, is ushered through several locked doors that lead to a corridor.

Letha is overwhelmed by the incessant noise - a cacophony of yelling, banging, clanging, and buzzing.

They pass a common room in which a group of INMATES are playing a heated game of cards.

A WOMAN (late 50s) in the common room spots LETHA through the window, and scurries into the doorway.

WOMAN

I know what you are!

This draws the attention of the other inmates who gather at the window and give Letha hardened stares.

Letha's face twitches, but she stares straight ahead.

INT. CORRIDOR OF CELLS - DAY

Letha is taken through a door and down a corridor of vacant cells.

Her eyes widen as she sees how small and sterile the cells are.

The guard stops outside a particular cell that is occupied. 
There are two INMATES inside - KIM (early 20s) is lying on the top bunk, and WILLOW (40s) lies on a single bed across from Kim, reading a book.

As the guard unlocks the cell, Kim leaps off the bed excited, and Willow briefly glances over the top of her book.

$$
\begin{array}{ll}
\text { I'm Kim. } & \text { KIM } \\
\text { Letha. } & \text { LETHA }
\end{array}
$$

Letha puts her hand out for Kim to shake, but Kim doesn't take it. She waves her hand instead.

$$
\begin{aligned}
& \text { KIM } \\
& \text { We're not meant to touch. } \\
& \text { GUARD } \\
& \text { Kim'll show you the ropes. }
\end{aligned}
$$

Kim looks proud to have been given the responsibility

Letha steps into the cell.

The guard leaves the cell door unlocked, and disappears down the corridor.

Letha and Kim stand stiffly in front of each other, unsure what to do.

$$
\text { Oh! That's ya bed. }
$$

Kim gestures to the bottom bunk.

KIM

$$
\text { They're more comfy than they look. }
$$

Letha nods her head, grateful.

$$
\begin{aligned}
& \text { KIM } \\
& \text { Actually, that ain't true. They're } \\
& \text { shit, but someone said that to me } \\
& \text { when I first got here, so... }
\end{aligned}
$$

Letha places her items on her bed and takes a seat. The bunk creaks and wobbles.

Kim leans against the cell door, fidgety.

$$
\text { So, what you in for? }
$$

Letha looks Kim up and down, relieved that she hasn't already heard. 
LETHA

A misunderstanding.

Kim nods, knowing.

$$
\text { I killed my stepdad. }
$$

Letha's gapes at Kim.

Kim does an uncomfortable dance on the spot to fill in the awkward silence.

Letha tries to catch Willow's eye, but Willow actively avoids her gaze.

Kim sees her looking in Willow's direction.

$$
\text { Everyone got their ways of coping. }
$$

Kim steps towards the bunk.

$$
\text { I talk to my baby girl mostly. }
$$

Kim pulls out a photograph from under her pillow, and shows it to Letha.

Letha has a hard time looking at the baby and its big brown eyes staring back at her.

$$
\text { LETHA }
$$

Lovely. What's her name?

\section{Sara.}

$$
\text { KIM }
$$

Letha hands the photograph back. Kim looks at it, sadly.

$$
\text { I wanna get back to her so bad. }
$$

She slips the photo back under her pillow.

$$
\text { KIM }
$$

I coulda had her in here with me, but I ain't want that for her.

Letha nods, understanding, but notices Willow subtly shaking her head to herself.

Kim then launches herself up onto the top bunk, causing Letha to bob up and down on her mattress. Kim swings her legs over the side, so that they dangle next to Letha's head. 


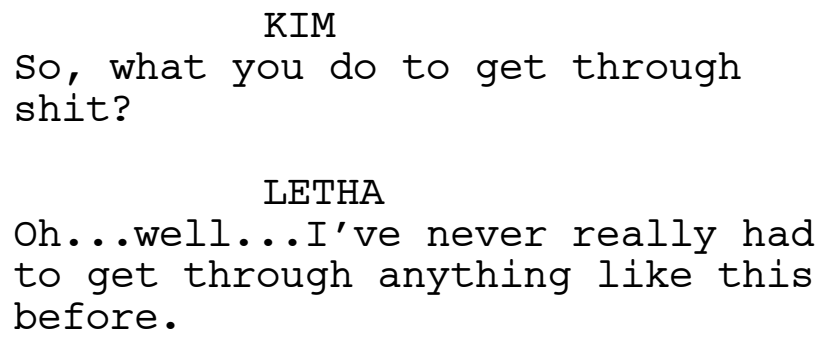

Letha laughs, apprehensive.

$$
\text { KIM }
$$

You should find something or you'll

lose ya goddamn mind.

Kim swings her legs back and forth.

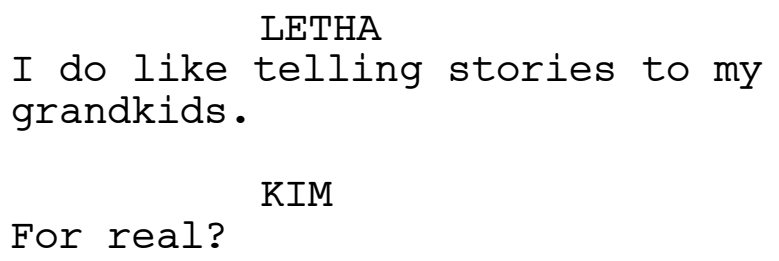

Kim flips her body around so that her head now dangles over the edge, and her upside-down head stares at Letha.

KIM

I love stories. Can you tell me one?

Letha is caught off guard. Kim sees her hesitation, and shrinks.

$$
\begin{gathered}
\text { KIM } \\
\text { Nahh. You right. Maybe later. }
\end{gathered}
$$

Kim lies back on her bed. There is a moment of silence between them, but the cacophony of prison sounds continue.

Letha lies down on her bed, uncomfortable. She stares up at the metal slats of Kim's bed - a loose thread from Kim's holey mattress dangles above Letha's head.

A loud buzzer goes off, causing Letha to jump in fright. Kim launches off her bed, and Willow puts her book down.

LETHA

$$
\text { What's that? }
$$

Kim turns to Letha.

\section{Dinner.}


INT. DINING HALL - NIGHT

The dining hall is abuzz with chatter and movement.

Kim, Willow and Letha are all lined up at a serving station with trays in their hands.

Letha's serving is slopped on her plate - it's unclear what the meal is exactly.

She goes to follow Kim to her table but Kim stops her.

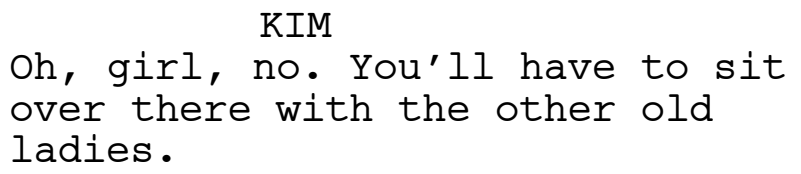

Kim points to a table across the room where a group of older women are sitting, including MEGAN - the woman who called her "baby killer".

Why?

LETHA

Kim shrugs.

KIM

People stick with their department, and the old ladies always get laundry cos it's the best job, so...

Kim takes a seat at her table.

Letha, uneasy, makes her way over to her table.

The other WOMEN glare at her as she takes a seat, but they don't say anything. Letha notices that she's the oldest by about ten years.

Letha tentatively tucks into her disgusting food in tense silence.

INT. CORRIDOR - NIGHT

Letha lingers behind the group of older women led by Megan. When they approach the common room they all file through the door. Letha hesitates, but decides to continue on.

MEGAN (O.S.)

Where you think you're going?

Letha stops and turns to Megan who is standing in the doorway to the common room. 
I'm a bit tired, so I thought I'd

just...

Letha gestures down the corridor.

MEGAN

We not good enough for you?

Megan stares threateningly at Letha.

Letha sees that Kim and Willow are both in the common room, so she makes her way through the door.

INT. COMMON ROOM - NIGHT (LATER)

Letha awkwardly watches a cheesy soap opera on the TV with several other inmates who are, unlike her, gripped by the show.

Megan is sitting with a younger group of particularly HARDENED INMATES.

ON THE TV SCREEN :

HOPE (early 20s), wearing an elegant gown and high heels, dangles from a rugged cliff face that overlooks an aggressive ocean far below. The background is clearly green-screened.

DANIEL ( 30 - a beefed up douchebag) reaches down to offer HOPE his hand. Hope grips it tightly, but Daniel doesn't pull her up.

Hope stares at Daniel, pleadingly.

DANIEL

will you marry me?

The inmates gasp in unison.

Hope is dismayed. She glances at Daniel, then at the deathly ocean below.

$$
\text { Girl, just } \stackrel{\text { KIM }}{\text { lie! }}
$$

Hope shakes her head slightly, and a darkness overcomes Daniel. He loosens his grip a little.

More gasps, and some groans, from the inmates.

$$
\text { I... }
$$$$
\text { HOPE }
$$

The inmates go silent in anticipation. 
But before they get to hear Hope's next words, the sound crackles and the image turns into static.

The inmates take a moment to realise what has happened.

$$
\text { INMATE }
$$

The fuck?

The inmates are overcome with rage, and verbally launch into each other and the guards.

Letha sits still in her chair as the tension around her escalates.

A chair is thrown across the room.

A GUARD, wielding two batons, stands up on a chair. He smacks the batons against each other, and screams at the top of his lungs.

$$
\begin{gathered}
\text { GUARD } \\
\text { SETTLE THE FUCK DOWN!! }
\end{gathered}
$$

The inmates eventually simmer down, and turn to him.

$$
\begin{gathered}
\text { GUARD } \\
\text { I'll get maintenance on it. Jesus. }
\end{gathered}
$$

The guard steps off the chair when he sees that everyone is under control.

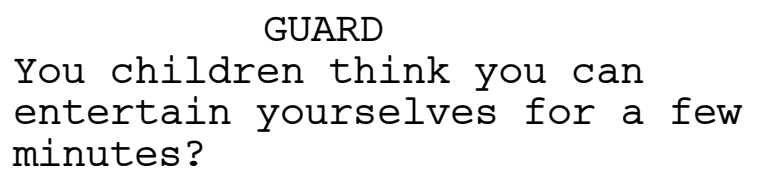

The guard steps out into the corridor, and speaks into his walkie-talkie.

The inmates all take their seats again, and sit in disappointed silence until one inmate - SASHA (20s) - stands up, claps her hands and rubs them together in glee.

$$
\text { SASHA }
$$

Let me tell yas a story.

Letha sits up, a little bit excited. But most of the inmates groan and boo at Sasha.

Sasha looks offended.

$$
\text { SASHA }
$$

Shut the hell up and listen, oi.

Sasha clears her throat. 


\section{SASHA}

Before I was locked up in this here shit hole me and my girls would get up to some hella tricks. This one night...

Sasha laughs just thinking about it.

$$
\text { SASHA }
$$

...this one night we--

CHANELLE (O.S.)

--got shitfaced and did something

fucked up.

CHANELLE (30s) has stood up.

Letha stiffens, shocked by the language. Megan watches Letha closely.

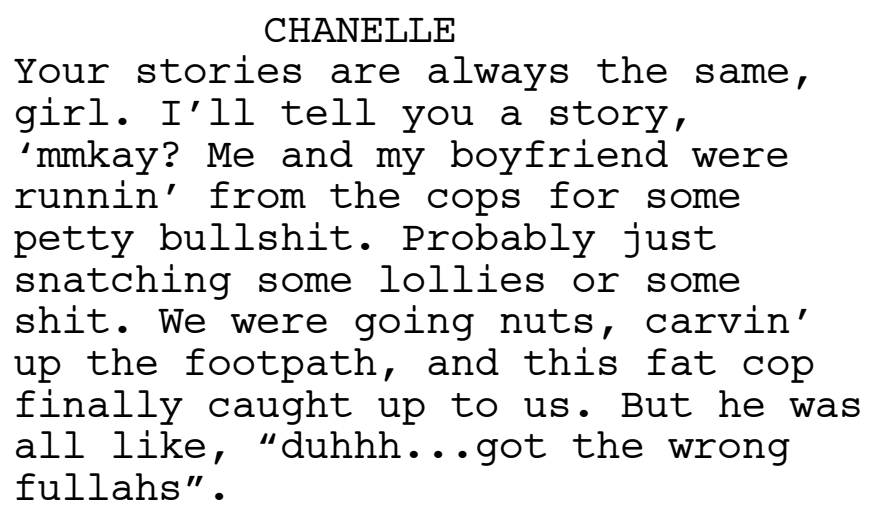

Chanelle cracks up this time, but no one else is laughing.

\section{CHANELLE}

Like, we were the crims but he

thought we wasn't.

Chanelle is annoyed that they're not finding this funny.

$$
\text { CHANELLE }
$$

Y'all suck balls.

Chanelle sits back down, and DES (40s) stands up.

$$
\text { DES }
$$

One time I cracked a girls head

open and saw her brains.

Everyone either looks grossed out or doubtful.

$$
\text { DES }
$$

It was an accident.

Des takes a seat, and Letha looks tempted to stand up. 
Kim catches her eye, and nods, encouraging her to stand up. Letha shakes her head, coyly.

Megan watches the strange interaction, curiously •

KIM

Letha told me she's good at stories.

Everyone's eyes fall on Letha. Megan looks excited for this opportunity.

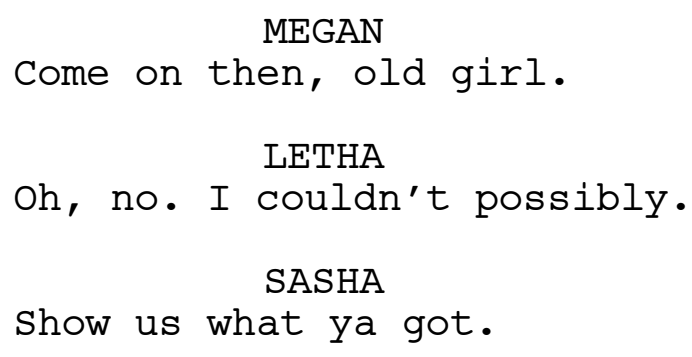

Letha can't contain her desire any longer.

LETHA

Well, alright then.

She stands up, and brings her hands together.

\section{LETHA}

You all seem to have experienced

some very troubling times, so I'll

tell you a story that's a bit

different, but not too different...

Letha winks at Kim, and the story flows out of her.

LETHA

There was once a remarkable young

woman named Sara.

Kim sits up straight, chuffed.

LETHA

She had a baby. No, that's not

right. She was pregnant. Yes.

Megan and the group of HARDENED INMATES whisper and laugh together. Letha tries her best to ignore them.

LETHA

Pregnant and on the run from some nasty people who were threatening to take her baby from her once it was born.

The hardened inmates' whispers get louder, until they're rudely talking over Letha. Letha speaks more emphatically to drown them out. 
She was running and running and

running, until she finally stumbled

upon a town...

Letha sees Willow in the corner, her nose still in the book.

LETHA

It was a medieval town.

Willow lowers her book to listen.

\section{LETHA}

And it was closing its gates

because there was a plague

spreading throughout the land and

the townspeople wanted to stop it

from reaching them.

At this, Megan and the hardened inmates all laugh at something. They pull themselves up and are about to leave, when Letha snaps and sternly turns to them.

\section{LETHA \\ Excuse me?!}

Megan and the hardened inmates suppress their laughter and turn back to Letha.

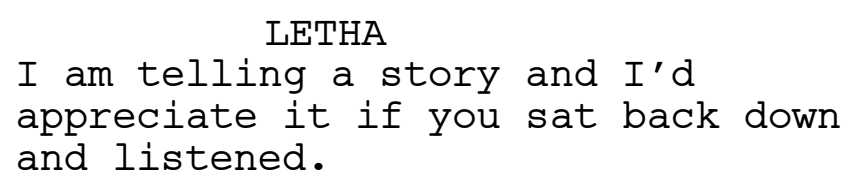

The room has gone silent. The group of inmates look perplexed.

Megan crosses her arms.

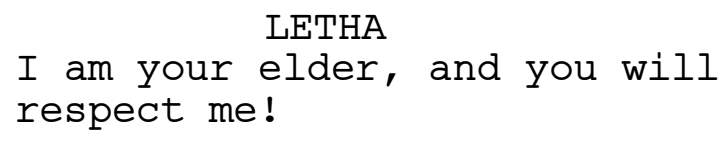

The group of inmates appear bamboozled by Letha's crazy outburst. But they clearly don't care. All of them march out of the room, leaving Megan behind.

Letha furiously watches as they exit.

Megan takes her seat again.

Once they've disappeared, Letha regains her calm composure and turns to the rest of the inmates who are all biting their tongues and sitting in uncomfortable silence.

Letha continues on as if nothing has happened. 
LETHA

Because the town was closing their

gates to stop the plague from

spreading, they wouldn't let poor

Sara in.

The inmates all stare at Letha - it's hard to tell if they're listening or just observing Letha's strange antics.

Only Kim and Willow seem genuinely enthralled by the story.

\section{LETHA}

She begged and pleaded for them to

let her in. "My baby. Please. I

need to save my baby. You're our

last hope," she cried.

The inmates raise their eyebrows at Letha's commitment to the story.

LETHA

Some people took pity on her, but a

horrible man, with missing fingers,

convinced them that they couldn't

risk letting the plague in for some

slut of a girl who got herself

knocked up.

There is a a small cheer from the inmates for Letha's use of the word "slut".

LETHA

Sara was ready to give up, when a handsome man, drawn to all the commotion, approached the gate. Upon hearing the situation, the handsome man - who just so happened to hold the highest position of power in the town - demanded that they grant Sara entry.

Kim's eyes light up, relieved.

LETHA

The handsome man offered Sara a bed in his home - a grand castle, that overlooked the town.

The inmates all let out a scandal-filled "oohhh".

Letha realises what they think she means, and corrects them horrified.

LETHA

He was a married man, with children

of his own. And she was pregnant! 
Some inmates laugh at Letha's prudishness, which Letha mistakenly takes as a compliment to her storytelling.

Letha continues on with even more gusto, even though no one, except Kim, looks invested.

\section{LETHA}

Sara went to bed that night, her belly full of food, and her mind finally at ease... But all that was about to change.

Kim frowns.

\section{LETHA}

The next morning, Sara awoke to panic and chaos....Everyone residing in the castle had been struck by the plague - the servants, the maids, the gardener, the children, the lady of the house, and the handsome man... They were all sick as sick can be.

\section{KIM}

What about Sara's baby?

LETHA

Her and her baby were fine. Healthy. Untouched.

Kim looks relieved again.

\section{LETHA}

But this was not good news. No, no, no... In the eyes of the townspeople, it made Sara look suspicious. Very suspicious indeed. With the handsome man on death's door, the horrible fingerless man appointed himself leader, and convinced the people that Sara was a witch! A witch who had brought the sickness upon those around her, sparing only herself.

Megan raises her eyebrows, unimpressed.

LETHA

Sara was captured by the townspeople - her arms and legs were bound by rope, and she was carried to a tower to await her fate... Here, a young boy, Thomas the fingerless man's son - kept watch over Sara. 
The guard from earlier lets a MAINTENANCE MAN into the common room. The INMATES all watch him as he clumsily makes his way over to the TV and inspects it.

Letha looks annoyed at the interruption, but persists trying to regain their attention.

\section{LETHA}

Thomas was the opposite of his

father, but he was determined to

prove to him that he was the same.

Thomas - seeing Sara's pregnant

belly - said, with pretend glee,

"Well, well, well. It looks like

two witches are going to be

executed tonight!"

Letha opens her mouth to continue, but is interrupted by clanging and banging as the maintenance man tries to jolt the TV back into action.

All the inmates, except for Kim, look slightly relieved by the interruption - much to Letha's annoyance.

When the clanging and banging finally stops Letha goes to speak, but then the TV static returns at full blast.

The maintenance man turns it down.

The guard checks his watch.

Letha opens her mouth again when--

$$
\begin{aligned}
& \text { GUARD } \\
& \text {--Alright. Leisure time's up, } \\
& \text { ladies. }
\end{aligned}
$$

Letha looks outraged, but she tries to conceal it with a smile.

The guard flicks the light on and off to get the lackadaisical inmates moving.

The inmates gather themselves and start filing out the door.

Letha lingers back, waiting for everyone else to go first. The flickering light makes her feel dizzy so she closes her eyes, and all goes dark.

We travel through Letha's darkness, and follow a tiny speck of light in the distance.

The light opens up into: 
INT. MEDIEVAL TOWER - DAY

The light flickers faintly as SARA (early 20s, played by Hope from the soap-opera) - pregnant belly, and arms and legs bound by rope - is slumped on the ground, unconscious.

Sara wakes again, and surveys her surroundings, confused. THOMAS (13) is slumped on the ground in front of her, unconscious.

\section{Hello?}

SARA

Sara tries to move but realises she's tied up. She panics.

\section{HELLO? !}

SARA

Thomas doesn't move.

Sara tries to struggle out of her ties, to no avail.

She shuffles herself over to the locked door, and manages to pull herself up onto her feet. She yanks and shakes the padlock, but it doesn't budge. She slams her bound fists against the door.

\section{SARA}

HELP ! !

INT. TOWER STAIRCASE - DAY

Sara's voice and knocking echoes down the spiral staircase.

EXT. TOWER - DAY

Sara's cries can't be heard outside.

A MEDIEVAL GUARD is slumped, asleep, on the ground outside the entrance to the tower.

EXT. MEDIEVAL TOWN - DAY

Throughout the town, people are slumped and unconscious in various positions.

A gallows is half-assembled in the town centre.

A group of UNCONSCIOUS OFFICIALS are scattered around it including the FINGERLESS MAN.

In the distance the tower that Sara is trapped in sits on a small hill, next to a grand castle.

Beyond the hill, the surrounding landscape is pixelated. 
The pixilation gradually spreads throughout the town.

When the town is entirely pixelated it all disintegrates into sand.

INT. CORRIDOR - NIGHT

Kim and Letha walk down the corridor together, behind the others.

A couple of GUARDS guide them all towards their cells.

Megan appears behind Kim and Letha.

KIM

Liked ya story.

Letha smiles, pleased.

LETHA

It's nice to be able to give you

girls something a bit different.

Megan scoffs.

MEGAN

I saw you judging them and their

stories.

Letha and Kim stops and turn to face Megan.

MEGAN

You know that what you did is way

worse than all their shit put

together, right?

Letha and Kim stop walking, and turn to face Megan.

LETHA

May I ask what your problem is?

MEGAN

My problem is that you killed your

own babies and you don't seem to

give a shit.

Kim gawks at Letha - seeing her in a new light.

What?

KIM

Letha clenches her hands, and shakes her head.

LETHA

You don't know anything about me. 
MEGAN

Does it make you feel better?

Convincing yourself that your

babies died naturally, like how

those people in your story were

killed by the plague?

Letha turns, and starts walking away.

MEGAN

If you ask me - Sara is a witch and

she deserves to be killed.

Letha is red in the face, and continues twitching, but she keeps walking.

\section{MEGAN \\ Burn the witch!}

Letha snaps.

She turns, and launches herself towards Megan who manages to side-step her, causing Letha to fall flat on her face and skid across the lino floor.

Megan is completely taken aback by the level of Letha's rage.

One of the guards, who missed the main action, goes to

Letha's aid. He lifts her up.

Letha tries to look like she's not in any pain.

Megan looks guilty for pushing her too far.

\section{What happened?}

Letha and Megan stare at each other.

MEGAN

I think she slipped or something.

The guard looks to Letha for confirmation.

LETHA

I get wobbly on my feet sometimes.

The guard nods, and lets Letha go.

Letha turns and limps off as fast as she can, leaving Kim and Megan behind.

$$
\begin{aligned}
& \text { KIM } \\
& \text { She for real killed her babies? }
\end{aligned}
$$

Megan hesitates. Her usual hostility is replaced with a hint of sympathy. 
Yeah.

MEGAN

Kim is flummoxed.

INT. CELL - NIGHT

The lights are out, but the cacophony of prison noises continue.

Willow is asleep on her bed.

Kim is on the top bunk, facing the wall, clutching the photo of her baby and stifling tears.

Letha is lying on her back, wide awake.

She stares up at the bottom of Kim's mattress, and notices the loose thread dangling above her. She pulls at it, but it doesn't budge.

She twists it around her wrist, cutting off the circulation to her hand. She looks determined to cause herself pain - her pupils widen.

We travel into the blackness of her pupils, and follow the speck of light again.

The light opens up into:

EXT. DESERT - DAY

Sara, still pregnant but no longer bound by ropes, is sitting in the middle of a vast desert completely petrified.

She sees a faceless figure scurry across a sand dune.

Sara stands up to get a better look, but the figure runs so fast that it turns back into sand.

From the sand another faceless figure appears. At first he walks and then he jogs, then sprints until he too turns into sand.

Another figure starts to form when Sara notices an object in the distance; the thing that the faceless figures seem to be trying to get to.

As Sara runs across the sand towards it, a wave of sand approaches her from behind.

She screams and sprints as fast as she can towards the object, but the wave of sand subsumes her. 
Sara is knocked to the ground, and everything is hazy but unlike Finley, the girl from the start - Sara retains her form.

The sand settles, and Sara finds herself on the lawn of the mansion from Finley's story.

EXT. MANSION - EVENING

The mansion is weather-beaten and far less idyllic than it was in Finley's story. It looks like it could be haunted.

Sara stands up, and makes her way to the front door.

She knocks, but doesn't get an answer.

She hears a kerfuffle going on inside, so pushes the door open and lets herself in.

INT. MANSION, STAIRCASE - EVENING

Sara makes her way up a wooden staircase. The faded walls are covered in strange, abstract paintings.

She reaches the upstairs landing, which leads to several lavish rooms.

INT. MANSION, LIBRARY - DAY

Sara creeps up to a doorway to the library where the voices are coming from.

She pokes her head around the corner, and sees LETHA 2, MALCOLM 2, EMILY 2, CHARLOTTE 2 (all off-kilter versions of the originals) as well as PHILIP MAVEN (40s) and ROSE MAVEN $(40 s)$.

They are all helping JULIAN 2 who has tried to hang himself from the ceiling with an enlarged version of the loose thread from Letha's prison cell.

Letha 2 is the one to finally get Julian 2 down, and he slumps on the floor, coughing and spluttering.

Emily 2 spots Sara in the doorway, and walks inquiringly towards her. Sara remains frozen on the spot, staring at Emily 2. No one else notices either of them.

MALCOLM 2

What the hell are you playing at, Dad?

Letha 2 inspects the thread, panic-stricken. 
LETHA 2

Where did you get this from?

Everyone looks to Julian 2 for an answer. Julian 2 splutters, distraught.

\section{JULIAN 2}

It just appeared, and I

couldn't... I couldn't stop it from

happening.

Julian 2 rubs his raw neck.

None of the adults seems to care that he's clearly in pain and distress.

Letha 2 grips the thread tightly.

PHILIP

I don't think Letha's coping.

Rose agrees with Philip.

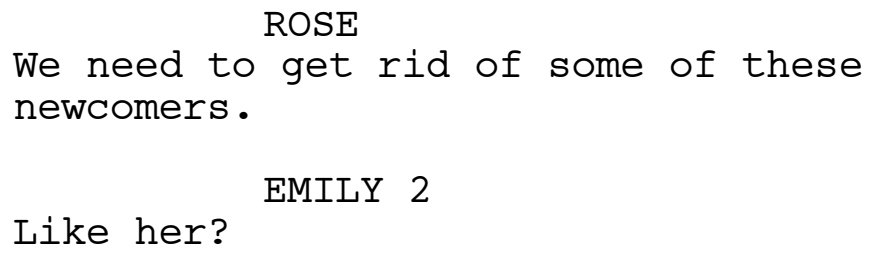

Emily 2 is pointing to Sara, who tries to hide behind the doorway, but cringes, knowing she's too late.

Who are you?

LETHA 2

Sara steps back into the doorway.

I don't know.

SARA

The adults all glance at each other, confused and alarmed. Letha 2 steps towards her, impatient.

LETHA 2

What's your name?

Sara shakes her head, muddled.

I don't know.

\section{SARA}

LETHA 2

Have you been inducted?

Sara shakes her head again and shrugs - not knowing what this means. 
SARA

I woke up in a desert, and then I

was here.

They all look at each other again, completely awed.

Julian 2 and the girls are the only ones who look pleased about whatever Sara's words mean to them.

Letha 2 inspects Sara closely. She's perturbed by her pregnant belly.

Emily 2 jumps up and down, excited.

EMILY 2

I've never seen someone from a story before!

Emily 2's jumping shakes the room slightly.

Emily!

MALCOLM 2

Emily 2 stops jumping, disheartened.

EMILY 2

What's it like?

SARA

I don't understand.

Letha 2 goes to the window and looks at the sky outside it's getting dark.

Below, at the back entrance to the mansion, Letha 2 observes a line of PEOPLE waiting to board a horse-and-carriage.

LETHA 2

Charlotte - put her on the carriage

for night shift. We'll figure out

what to do with her when she gets

back.

Julian 2 looks concerned about this, and clearly wants to say something, but he refrains.

Charlotte 2 begrudgingly marches over to Sara.

Follow me.

CHARLOTTE 2

Sara follows Charlotte 2 down the corridor, and overhears the others all anxiously jabbering at Letha 2 until she snaps:

LETHA 2 (O.S.)

Everyone just needs to calm down! 
INT. DOWNSTAIRS, CORRIDOR - EVENING

Charlotte 2 and Sara pass by a kitchen, where CHIEKO 2 is working hard at preparing a large meal.

Charlotte 2 takes Sara around a corner, down a corridor filled with dreary-looking bed-chambers.

Many of the doors are closed, but some of them are open and Sara makes eye contact with their occupants - off-kilter versions of the INMATES, as well as PEOPLE we haven't met yet - including a young boy, SIMON (9) who makes a blanket fort in his room.

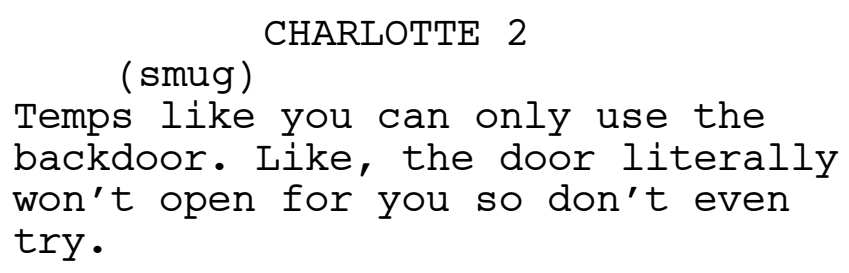

Sara frowns.

\section{SARA}

I just came through the front door.

Charlotte 2 looks puzzled by this.

Oh.

CHARLOTTE 2

Charlotte 2 shrugs it off, and leads Sara towards the back door anyway.

Charlotte 2 pushes it open.

EXT. MANSION, BACK GARDEN - NIGHT

The back garden is vast, but overgrown and untended to. A shabby outhouse is embedded in a tangle of dead branches.

The horse-and-carriage is still there. HEADMASTER (60s, strict) carries a whip and hops onto the coachman's seat of the carriage.

The people who were lining up are now filing into the backseat of the carriage.

CHARLOTTE 2

Find a seat with the others.

SARA

Where is it going?

Letha's dreams.

CHARLOTTE 2 
Sara frowns.

Who's Letha?

SARA

Charlotte 2 laughs like this is an absurd thing to have to ask.

CHARLOTTE 2

Oh, yeah. Make sure you don't bring anything back with you.

Charlotte 2 gives her a little wave before heading back inside.

Sara turns around, and tentatively makes her way onto the carriage.

INT. CARRIAGE - NIGHT

The carriage is crammed with about eight people - GREGORY 2, the JUDGE 2, JUROR 2, FOREPERSON 2, MEGAN 2, KIM 2, and a few STRANGERS .

Sara can't find anywhere to sit, until Megan 2, who has a warm and welcoming vibe to her, moves over and gestures for Sara to sit.

$$
\begin{gathered}
\text { MEGAN } 2 \\
\text { Are you OK? } \\
\text { SARA } \\
\text { I feel really... }
\end{gathered}
$$

Sara struggles to find the right words.

MEGAN 2

know exactly what you mean.

Megan 2 gently holds Sara's hand to comfort her.

MEGAN 2

We'll look out for each other, OK?

Megan 2 smiles at Sara, and Sara looks a little more at ease.

Outside, the horse whinnies as it is whipped. The horse begins to trot.

The carriage starts to move.

INT. PRISON, CELL - NIGHT

Letha, exhausted, has released her wrist from the thread. 
Her head is resting on her pillow, and her eyes are drooping as she nears sleep, when she hears a series of blood-curdling screams echo from down the corridor - someone having a terrible dream.

It wakes Letha up again, sending a shiver down her spine.

EXT. MANSION, DRIVEWAY - NIGHT

The horse-and-carriage is heading for the mansion's gated entrance when the horse gets spooked and whinnies again. It stops in its tracks.

INT. CELL - NIGHT

The blood-curdling screams continue, so Letha curls her pillow over her head, until she starts to gradually drift off to sleep again.

EXT. GRAVEL ROAD - NIGHT

The horse is back in action, and pulls the carriage down a gravel road that seems to go on forever.

INT. CARRIAGE - NIGHT

Sara squints out the window, trying to see where she is, but it is too dark to make anything out, and eventually the darkness is smattered with bursts of colour and patterns.

INT. CELL - NIGHT

Letha has gone to sleep, clenching her pillow and frowning.

INT. CARRIAGE - NIGHT

The horse-and-carriage is moving so fast it almost seems like they're flying.

Sara continues to stare out the window in awe, lost in the swirling patterns and colours that form outside.

When the horse-and-carriage slows to a stop, Sara turns and sees that the other passengers, other than Gregory 2, have disappeared.

GREGORY 2

Are you good at maths?

Sara shakes her head. 


$$
\begin{aligned}
& \text { GREGORY } \\
& \text { That's too bad. }
\end{aligned}
$$

Gregory hops out of the carriage.

Sara follows after him.

EXT. UNDERGROUND

Sara exits the carriage and finds herself in a tiny burrow beneath the ground. Gregory 2 is squatting, ahead of her.

GREGORY 2

Told you you're gonna need maths.

Gregory 2 crawls through the burrow on his hands and knees.

Sara turns around to get back on the carriage, but it's gone.

She's surrounded by dirt - there's no where else to go but where the burrow leads her.

She is crawling behind Gregory 2, when they both hear bloodcurdling screams - like those of the inmate having a nightmare.

Gregory 2 and Sara crawl faster to get to the source of the screams.

The burrow gradually widens, until they're both able to stand, and are met by some of the people who disappeared from the carriage - Kim 2, Foreperson 2, Juror 2, and a stranger. They look terrified to see them.

$$
\begin{gathered}
\text { FOREPERSON } 2 \\
\text { Who are you? } \\
\text { STRANGER }
\end{gathered}
$$

You shouldn't be here.

\section{GREGORY 2}

We're looking for the solution to

the maths problem.

\section{SARA}

Why were you screaming?

The water!

FOREPERSON 2

Foreperson 2 gestures to the dirt beneath their feet.

JUROR 2

It's filling up fast.

What water?

SARA 
Water starts to pool at their feet.

KIM 2

We have to keep going or we'll burn

to death.

Sara frowns, confused. The four of them march past Gregory 2 and Sara.

SARA

It's a dead end down there.

They stop in their tracks.

FOREPERSON 2

We'll keep digging our way through.

SARA

Why don't we dig upwards?

Gregory 2 looks impressed.

GREGORY 2

You said you weren't good at maths!

The others look confused at this proposition.

FOREPERSON 2

What is upwards?

SARA

You know, upwards.

Sara points up. The others laugh, like this is the most

ludicrous thing they've ever heard. But then they see that

Sara is being serious, and their laughs are subsumed by fear.

FOREPERSON 2

That's where the bad people are

sent.

A wave gushes through the burrow, raising the water-level to their knees.

SARA

I thought this is where bad people are sent.

The others laugh.

$$
\begin{gathered}
\text { JUROR } \\
\text { Some education you got, lady! } \\
\text { FOREPERSON } 2 \\
\text { You must be a psychopath. }
\end{gathered}
$$

Everyone but Gregory continue marching on towards the dead end. 
The water rises some more, without a wave this time.

Sara starts to panic. She ducks into the narrower part of the burrow, and starts digging upwards on a slight diagonal, with her hands. She makes very little progress.

\section{Here.}

GREGORY 2

Gregory 2 hands Sara a plastic teaspoon. Sara takes it gratefully, and continues digging with it. The teaspoon is much more productive - doing most of the work for Sara.

She crawls up into the space as she digs it out.

Soon she reaches the earth's crust. She breaks through the surface, and a piercing light descends into the underground.

Sara looks back down the tunnel to call out to Gregory 2 and the others, but the underground has disappeared, and she now looks down at a roaring ocean and realises she's hanging precariously from a cliff.

$$
\text { Help! }
$$

SARA

At that, CHARLOTTE 3 and EMILY 3 - their skin sickly blue come running for Sara.

Sara is overcome with emotion at seeing them, but is grossed out by their blue skin.

SARA

What's wrong with you?

They hold out their hands to pull Sara up. Sara grabs onto Charlotte 3's hand, but instead of pulling herself up, she tugs at Charlotte 3's arm, causing her to fly off the cliff to her death.

Emily looks panicked and tries to pull her hand away, but Sara grabs it and flings her off the cliff too.

Sara pulls herself up until she's safe on land. She stares down at the ocean and bursts into uncontrollable sobs.

$$
\text { Not again... }
$$

Sara turns to see the most beautiful TREE she's ever seen, hanging over her. The sight of it fills her with comfort and glee.

Sara goes over to the tree and hugs its trunk.

SARA

Thank you for coming. 
The tree chuckles, like a friendly old man.

TREE

You've got to stop doing that.

Doing what?

SARA

TREE

Tossing your loved ones over the cliff.

Sara is crestfallen, and pulls herself away from the tree. SARA

I didn't!

TREE

I saw what I saw. And I've seen what I've seen.

Stop lying!

SARA

TREE

How can you accuse me of lying when

you don't even remember what just

happened?

The tree whacks Sara in the head with one of its branches. Sara's eyes glaze over.

Sara desperately hugs the tree again for comfort, but it shrinks in her grasp until it is a shrub, and then a seedling, and then a seed that sits in the palm of Sara's hand.

Sara grips it tightly and puts it in her pocket.

She sees a light in the distance, and runs towards it until she finds herself back at the carriage.

INT. CARRIAGE - NIGHT

Kim 2, Gregory 2, Judge 2, and the jurors are all there, looking frazzled and exhausted.

Sara, also frazzled, takes a seat.

KIM 2

That was nuts.

Everyone nods, agreeing.

STRANGER

I've done this hundreds of times, and it's never been that twisted. 
Sara lowers her voice, guilty.

SARA

I...I killed those two little girls

who were back at that house.

Judge 2 puts his hand on Sara's knee.

JUDGE 2

None of that was real.

Sara processes this, unsure whether to be relieved or not.

SARA

It felt real.

JUDGE 2

That's because Letha's just been

sent to prison for killing two of

her babies.

JUROR

But she didn't really do it, it's

just fresh in her mind is all.

Sara is about to ask a question when the stranger enters the carriage, followed by Megan 2 who looks calm and refreshed compared to the others. They both take a seat.

KIM 2

Why aren't you messed up like us?

MEGAN 2

I just came from...

Megan 2 shakes her head, disbelieving. She pauses, a coy expression on her face.

MEGAN 2

...well, I was cuddling with Letha

on a golden beach.

Everyone, but Sara, looks surprised by this.

MEGAN 2

It was pure bliss.

Sara's confusion mounts.

Who is Letha?

SARA

Headmaster appears in the door of the carriage, carrying an industrial torch.

MEGAN 2

Were you not given the induction

when you got here? 
Sara shakes her head.

Headmaster switches the torch on and shines it into the carriage.

\author{
HEADMASTER
}

Security check, folks.

Everyone in the carriage stands and heads outside.

EXT. CARRIAGE - NIGHT

Everyone is lined up outside the carriage.

Headmaster shines his heavy-duty torch at everyone as he speaks.

\title{
HEADMASTER
}

I need you all to strip off.

Everyone gawks, not moving .

HEADMASTER

We got orders from the top to

tighten security.

The others all look at each other in horror.

\section{HEADMASTER}

It's the only way we can be sure

you don't take any contraband back

with you.

Sara remembers the seed. She slips her hand in her pocket and feels it.

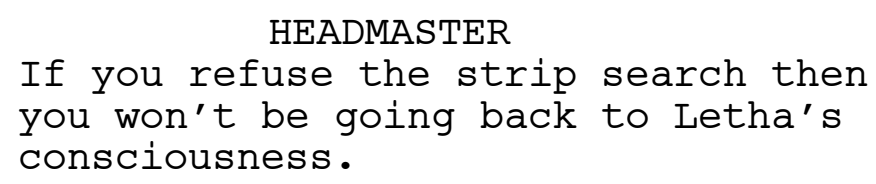

The others all hesitantly strip off.

Sara is frozen on the spot, struggling to take all this in.

Headmaster aggressively shines the torch on Sara who then tentatively peels off her clothes.

Headmaster inspects everyone, one by one - closely examining their bodies, and patting down their clothes, similar to how Letha was inspected by the prison guard.

When Headmaster gets to Sara she asks:

\section{SARA}

Why can't we take anything back? 
Headmaster shines the torch right in her eyes.

$$
\begin{gathered}
\text { HEADMASTER } \\
\text { You have something on you? }
\end{gathered}
$$

Sara hesitates, then shakes her head.

Headmaster doesn't trust her - he inspects her body closely and pats down her clothes.

He doesn't notice the seed. Sara relaxes a little.

$$
\begin{aligned}
& \text { HEADMASTER } \\
& \text { Taking back contraband can result } \\
& \text { in Letha remembering her dreams. }
\end{aligned}
$$

Headmaster picks Sara's clothes up for her, but before handing them over says, threateningly:

HEADMASTER

And you don't wanna know what might

happen if Letha remembers her

dreams.

Sara takes her clothes back, and Headmaster moves on to the next person in line.

Sara puts her clothes back on, slips her hand in her pocket to feel the seed again.

She considers tossing the seed out, but decides against it and gets back into the carriage.

INT. CARRIAGE - NIGHT (LATER)

Sara stares out at the darkness through the window, as they travel back down the gravel road.

EXT. MANSION, BACK GARDEN - NIGHT

The horse-and-carriage pulls up outside the mansion, which is dark, quiet and still.

Everyone quietly files out of the carriage, and makes their way through the back entrance.

As Sara hops off the carriage, she pries the seed out of her pocket and tosses it into the weeds of the back garden.

Kim 2, right behind Sara, sees this but doesn't say anything. They both make their way inside. 
INT. MANSION, DOWNSTAIRS CORRIDOR - NIGHT

Everyone walks down the corridor and disappears into their allocated bedchambers.

Sara stops in her tracks, realising she doesn't have an allocated room.

Kim 2 stops outside her room, and is about to go inside when she sees Sara standing there.

KIM 2

Wanna bunk with me?

Sara nods, grateful. She walks over to her.

KIM 2

I have a weird roommate who doesn't talk.

Kim 2 opens the door.

INT. BEDCHAMBER - NIGHT

The room is tiny and dusty, and only has one single bed.

WILLOW 2 is asleep on a make-shift bed made out of blankets on the floor.

KIM 2

They said the rooms are all full up at the moment.

Kim 2 gets into the bed, and gestures for Sara to get in next to her.

Sara slips into bed. The two are silent for a while. Sara closes her eyes, while Kim 2 remains wide awake.

Kim 2 whispers to Sara.

KIM 2

I saw you.

Sara opens her eyes again, and whispers back.

What?

SARA

KIM 2

Flicking something into the garden

just now. What was it?

Sara hesitates.

SARA

You promise not to tell anyone? 
Kim nods.

SARA

It was a seed from Letha's dream.

Kim 2 sits bolt upright.

KIM 2

Are you nuts?

Sara is taken aback by Kim 2's outrage.

KIM 2

Why the hell would you do that?

SARA

To see what happens.

Kim 2 gawks at Sara like she's a lunatic.

SARA

Aren't you curious?

Kim 2 laughs, outraged.

KIM 2

No.

Kim 2 lies back down, and turns over.

KIM 2

Your funeral.

Sara looks a little uneasy, but clearly can't understand what her problem is.

INT. PRISON, CELL - MORNING

Letha wakes up to a loud buzzer going off. She rubs her head as if she has a headache. She looks completely out of sorts.

Willow and Kim are waiting at the cell door.

$$
\text { Morning. LETHA }
$$

Neither Willow nor Kim respond.

A GUARD approaches and unlocks their cell.

Willow and Kim disappear down the corridor.

Letha pulls herself out of bed, head throbbing. 
INT. PRISON, DINING HALL - MORNING

Letha, carrying her breakfast tray, makes her way to the table that Megan and company are sitting at.

Letha and Megan glance at each other, awkward.

Letha tucks into her porridge.

Letha stops mid-chew, and whispers to Megan.

\section{LETHA}

Can I talk to you sometime?

Megan frowns, but flicks her head up - agreeing.

INT. MANSION, BEDCHAMBER - MORNING

Kim 2 shakes Sara awake, panicked.

KIM 2

Your fuckin' seed, man.

Sara is confused and groggy.

EXT. MANSION, BACK GARDEN - MORNING

A crowd of people gather at the back entrance, gawking.

Sara and Kim 2 push their way through the crowd.

Sara stares up at the now-gigantic tree that has clearly grown from her seed, and continues to grow, uncontrollably, right before their eyes.

A couple of MEN, wielding axes and chainsaws, are led by Philip as they try their best to cut the tree back. It's a losing battle.

KIM 2

(to Sara)

Still curious?

A particular branch grows so big and thick that it impales a TEENAGED GIRL in the crowd, before crashing into a wall of the mansion.

Everyone in the crowd gasps and screams. Some people sprint away in fear.

Philip lops the branch off, which causes the tree to let out a horrendous screech of pain before shooting out more branches that ascend into the sky.

Sara curiously surveys the horrified expressions on everyone's faces. 
INT. MANSION, SITTING ROOM - MORNING

Letha 2, Rose, Julian 2, and Malcolm 2 are at the window, anxiously watching Philip and the other men continue to hack at the tree. Its ascending branches start to curve as if its intention is to envelop the mansion.

Letha 2's panic rises.

LETHA 2

It's going to encase us.

Letha 2 turns to Malcolm 2 .

LETHA 2

Go help Dad.

Malcolm 2 scurries out of the room.

ROSE

You need to find out who did this,

Letha.

Letha 2 surveys the crowd.

ROSE

You need to send them a message.

Letha 2 spots Sara standing in the crowd, surveying everyone else.

LETHA 2

That girl who came from the story.

Letha 2 turns to the others - a fire in her eyes.

Julian 2 looks extremely alarmed by Letha 2's suspicion of Sara, but tries to conceal it.

JULIAN 2

Weird things were happening well

before she arrived.

ROSE

Maybe weird things were happening

because she was arriving.

Letha 2 nods, convinced. Julian 2 shakes his head, panicked, then blurts out:

JULIAN 2

I know who did it.

Rose and Letha 2 can't believe what they're hearing.

JULIAN 2

One of your childhood classmates. I

caught him planting a seed. 


$$
\text { LETHA } 2
$$

Why didn't you say anything?

Letha 2 steps towards Julian 2, venom in her eyes. Julian 2 cowers.

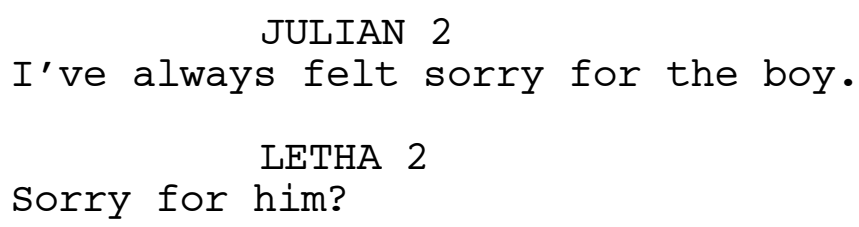

Letha 2 strikes Julian 2 across the back of the head. Julian 2 winces in pain.

$$
\text { Get him. LETHA } 2
$$

Julian 2 vigorously shakes his head.

$$
\begin{aligned}
& \text { JULIAN } 2 \\
& \text { Please don't make me. } \\
& \text { LETHA } 2 \\
& \text { Get him, Julian. }
\end{aligned}
$$

Julian 2 bows his head, and treads out of the room.

INT. PRISON, LAUNDRY ROOM - DAY

Letha is folding sheets with Megan. Beadie and the other women are in an adjoining room, retrieving laundry from the driers.

Letha is lost in thought.

\section{So?}

MEGAN

Letha looks to Megan, questioningly.

MEGAN

You had something to say?

Letha smiles calmly, but doesn't say anything.

MEGAN

Look. I thought you didn't feel

guilty for what you did, but I now

know I was wrong.

Letha glances over at Megan. She speaks quietly, but there's a hint of glee in her voice.

LETHA

No you weren't. 
Megan looks confused.

$$
\text { I don't feel guilty. }
$$

Megan puts her sheet down, creeped out by Letha's demeanour.

$$
\text { I've never felt guilty. }
$$

Letha smiles at Megan, like she's proud of herself.

Letha blissfully continues to fold the sheet in front of her. Megan looks petrified.

EXT. MANSION, FRONT ENTRANCE - DAY

The tree has grown, and continues to grow, so that it now hangs over half of the mansion like a canopy, blocking out much of the natural light.

Several men are up on the roof, hacking at the branches and containing them with wire mesh. The tree lets out screeches of pain as they do so.

The high-pitched wailing of a petrified boy also fills the air.

Everyone, including Kim 2 and Sara, is now gathered at the front entrance.

Simon - the blanket-fort kid that Sara saw earlier - has two dangling ropes tied around his wrists and is being restrained by Julian 2 . Simon has tears streaming down his face.

Letha 2 stands next to them both. She screams into a loud speaker.

\section{LETHA 2}

This young boy is responsible for

the events today.

Sara frowns, trying to understand.

\section{SIMON}

Please! I didn't do nothing. I swear.

$$
\text { LETHA } 2
$$

Anyone who sneaks in contraband is to be executed immediately.

No!

SIMON 
Do we understand?

Letha 2 looks at the crowd of people. They all nod, petrified.

Kim 2 glares at Sara who is also nodding. Sara is more confused than terrified.

Letha 2 gestures to Julian 2 .

$$
\begin{aligned}
& \text { JULIAN } 2 \\
& \text { (whisper) } \\
& \text { I'm so sorry. }
\end{aligned}
$$

Julian 2 walks Simon across the lawn.

Tears stream down Simon's face.

SIMON

Does this mean she'll forget me?

Julian 2 slumps a little.

$$
\text { Maybe not forever. }
$$

Simon whimpers, petrified.

When they're in the middle of the lawn, and have enough space around them, Julian grabs hold of the two ropes.

Julian proceeds to spin Simon around and around until he is airborne.

Eventually Julian 2 lets Simon go, and he is propelled into the air.

Simon screams in terror and pain as his body is torn into little pieces that then turn into sand before vanishing into thin air.

Julian 2 looks pained and dizzy.

Letha 2 eyes the crowd sternly.

$$
\begin{gathered}
\text { LETHA } 2 \\
\text { Zero tolerance! }
\end{gathered}
$$

All the onlookers are gobsmacked.

Kim 2 glares at Sara again.

KIM 2

You did that.

Sara looks furious. 
Kim 2 turns and walks away. Sara watches as Rose joins Letha 2 and Julian in an exclusive huddle.

INT. PRISON, LAUNDRY - DAY

Megan has abandoned Letha, and joined the others in the adjoining room.

Letha continues to fold the laundry as she hums a little tune to herself.

A GUARD enters.

$$
\text { Maven? GUARD }
$$

Letha looks up.

Yes?

$$
\text { LETHA }
$$

GUARD

Phone call.

The others tentatively look over at Letha, who follows the guard.

$$
\text { Told it's a family emergency. }
$$

Letha doesn't appear alarmed by this. She continues humming.

EXT. MANSION, FRONT ENTRANCE - DAY

Everyone, traumatised, trudges down the path to the back of the house.

Sara pushes through the crowd to catch up with Kim 2 .

$$
\text { Kim! }
$$$$
\text { SARA }
$$

Kim 2 walks faster to get away from her, and rounds the corner.

EXT. MANSION, BACK GARDEN - DAY

Kim 2 passes by the tree, and is heading towards the outhouse.

Sara, annoyed, runs in front of her, and walks backwards as she speaks urgently to her. 
SARA

We have to do something.

Kim 2 tries to dodge Sara, but Sara pushes Kim 2 into the outhouse, before stepping inside herself and slamming the door closed.

INT. BACK GARDEN, OUTHOUSE - DAY

Sara stands against the door so Kim 2 can't get out.

KIM 2

What the hell?

SARA

I'm sorry. It's just...that dream I

was in last night where I killed

those girls? It felt really real.

KIM 2

So?

Sara looks bewildered that Kim 2 doesn't seem to get it.

SARA

Letha's a bad person.

KIM 2

No she's not.

Kim 2 tries to get past Sara, to no avail.

SARA

We need to get out of here.

KIM 2

Yes. Please.

Kim 2 tries to push Sara out of her way again, but Sara stands her ground.

Kim 2 looks Sara dead in the eyes.

KIM 2

There is no out of here.

Sara lets the weight of this statement sink in.

SARA

There has to be.

Kim 2 shakes her head at Sara like she's an idiot.

Kim 2 uses all her might to push Sara out of her way, and finally tumbles out of the outhouse.

Sara calls out after her. 
SARA

Well, that's messed up and we need

to make Letha pay for trapping us

in here.

Kim 2 scurries into the mansion.

Sara steps outside, rattled but determined.

EXT. MANSION, BACK GARDEN - DAY

Philip, trying to hack away at the trunk of the tree, spots Sara and eyes her suspiciously.

PHILIP

You been given a job yet, girlie?

Sara shakes her head.

INT. PRISON, PHONE BOOTH STATION - DAY

The guard guides Letha to one of the free phones, that has a light flashing.

Letha picks the phone up.

$$
\begin{aligned}
& \text { Hello? } \\
& \mathrm{Hi}, \mathrm{Ma} .
\end{aligned}
$$

Letha takes a moment to consciously shed her blissful expression and demeanour and replace it with her frail grandmotherly façade.

$$
\text { LETHA }
$$

Malcolm pauses.

$$
\text { Oh. }
$$$$
\text { MALCOLM (PHONE) }
$$

Malcolm speaks quietly.

$$
\text { MALCOLM (PHONE) }
$$

I lied about the emergency, I just wanted to talk to you.

Letha grins a little.

$$
\text { How are you doing? }
$$


I'm good.

LETHA

Letha catches herself.

LETHA

I mean. You know, honey. Fine.

Considering.

Letha sees that guard is watching her closely. She lowers her voice.

$$
\begin{aligned}
& \text { LETHA } \\
& \text { I need to tell you something. } \\
& \text { MALCOLM (PHONE) } \\
& \text { Yeah? }
\end{aligned}
$$

Letha grimaces as she speaks.

LETHA

I haven't been honest with you.

About?

$$
\text { MALCOLM (PHONE) }
$$

Letha hesitates.

$$
\text { MALCOLM (PHONE) }
$$

Is it about the case? Cos that's

why I'm calling, actually.

Letha frowns, confused.

Oh?

$$
\text { LETHA }
$$

\section{MALCOLM (PHONE)}

Yeah. Gregory reckons he's found a a way to get you out.

Letha is injected with hope.

Oh?

LETHA

$$
\begin{aligned}
& \text { MALCOLM (PHONE) } \\
& \text { Yeah. He's working real hard. He's } \\
& \text { recruited some other lawyers who're } \\
& \text { experts in getting innocent people } \\
& \text { out of jail. }
\end{aligned}
$$

Letha clearly likes the sound of this.

$$
\text { MALCOLM (PHONE) }
$$

What were you going to say, though?

Is it something that could help us

with the case?

$$
\text { (MORE) }
$$


MALCOLM (PHONE) (CONT'D)

Cos I can handle anything you lay

on me if it'll get you out of

there.

Letha falls silent, considering.

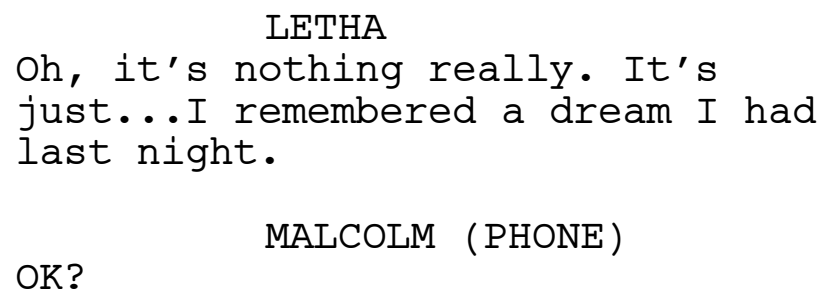

LETHA

I haven't remembered a dream since

I was a teenager.

Malcolm laughs, clearly confused.

LETHA

When you were a little boy you used to tell me your dreams every morning over breakfast, and I told you my dreams in return.

I don't remember that.

MALCOLM (PHONE)

LETHA

Well, I was making them up.

Malcolm falls silent.

LETHA

And I just wanted to apologise.

Letha waits tentatively for his response.

Malcolm laughs again.

MALCOLM (PHONE)

Ma, it's ok. We've got bigger fish

to fry, yeah?

Yes. Of course.

Letha smiles, perniciously.

LETHA

Let's get me out of here. 
EXT. MANSION, FRONT ENTRANCE - DAY

The tree has stopped expanding. The men continue to hack away at its branches. It is no longer emitting its screeches of pain.

INT. MANSION, LIBRARY - DAY

Sara is wearing an apron, and is being given instructions from Chieko 2 who is placing a step ladder in front of a tall bookcase, while holding a feather duster.

\section{CHIEKO 2}

I wanted to put you on laundry

since it's a bit more suited to

your condition.

Chieko 2 gestures to her pregnant belly.

\section{CHIEKO 2}

But Master Julian insisted, so... I'm sorry.

Chieko 2 hands over the feather duster.

CHIEKO 2

Make sure you get any sand.

Sara nods to show she understands, and Chieko 2 leaves her to it.

Sara steps up on the ladder and begins dusting the bookcase, when she notices the titles that are hand-written on the spines of the books. Things like "Emily and Charlotte", "Malcolm as a Baby", "Marriage with Julian".

Sara checks to see that the coast is clear, then takes a book down that is titled "Childhood: Vol 1".

She opens it up. It's a scrap book - pages filled with photographs, newspaper clippings, drawings, and letters, all accompanied by captions.

Sara flips to a page that has a photograph of a slightly younger-looking Philip and Rose holding a baby Letha: "Mum, Dad and Baby Letha"

Sara continues to flip through the pages when Julian 2 appears in a door to an adjoining room in the far side of the library.

Sara gasps and snaps the book shut. She tries to act natural but has clearly been caught out.

$$
\text { It's OK. }
$$$$
\text { JULIAN } 2
$$ 
Sara puts the book back, and continues dusting - mortified.

JULIAN 2

I was wanting to catch a moment

alone with you.

Sara looks panicked. Julian 2 winces at her obvious fear of him.

JULIAN 2

Please don't be scared of me.

Sara sees the look of sad desperation in his eyes, and relaxes a little.

JULIAN 2

I need your help.

Sara is curious.

Julian 2 checks to see no one is around, then steps closer to Sara who remains elevated by the ladder.

Julian lowers his voice.

JULIAN 2

It's just... Letha's creative endeavours usually arrive in abstract forms.

Julian 2 gestures to the paintings on the walls.

Sara surveys the paintings, and stops on a particular one which is slightly more detailed, and fresher, than the others - the vague outline of a young girl and a cat.

JULIAN 2

The fact that you made it through

to Letha's consciousness as...

Julian 2 gestures awkwardly at Sara's body •

JULIAN 2

...you. Well....It's highly unusual.

Sara blinks, trying to comprehend.

JULIAN 2

But the few times someone like you

has managed to arrive, they've had

certain attributes that meant they

could access things that no-one but

Letha can.

Sara's eyes go wide. 
JULIAN 2

I need you to use your attributes

to destroy something in Letha's

long-term memories.

Julian 2 looks up at her, pleadingly.

SARA

Will it harm Letha?

Sara notices Julian 2 hesitating.

JULIAN 2

I'll stay behind to make sure it doesn't.

SARA

But it could harm her?

Julian 2 grimaces, guilty.

JULIAN 2
I mean, it's a risk, yeah.

I'll do it.

SARA

Julian 2 looks immensely relieved, but then shoots her a questioning look.

\section{JULIAN 2 \\ You want to harm her?}

Sara smiles, then goes back to dusting, She finds a small pile of sand from a half-deteriorated book in the back corner of a shelf.

She brushes the sand off the shelf with the duster, and it scatters in the air.

End of Episode. 
UNTOLD

Episode Two:

"Groundwork"

Written by

Alison McLachlan 

INT. RECORDING ROOM - DAY

A 1940s movie camera is set up on a tripod, and is filming images that are flickering on a screen in an otherwise empty and sealed-off room.

On the screen we see the following, all from Letha's perspective:

Letha's clenched hands resting on her lap under a table.

Letha glances across the room that she's in - the prison dining hall - and stares at Kim who is laughing with her COMPANIONS at her table.

Kim and Letha make eye-contact, but Kim quickly looks away.

Letha pulls herself up, and heads over to Kim.

Kim ignores her completely, but her table companions all glare at her like she's a weirdo.

\section{LETHA \\ Can we talk?}

Kim stares straight ahead.

One of Kim's companions - GRETA - speaks for Kim.

\section{GRETA}

She don't want nothing to do with you.

Why not?

$$
\text { IETHA }
$$

GRETA

I think you know.

Letha looks aggravated.

\section{LETHA}

I wish you'd just hear me out, so you can make an informed decision about me rather than just assuming things.

Kim frowns, but doesn't break her silence.

LETHA

I don't judge you for killing your stepdad!

Kim sits up straight, offended.

Woah!

GRETA 
Greta, and some of Kim's other companions stand up, threatening.

Letha sees that a A NEARBY GUARD keeps his eye on the action, ready to step in.

Kim's companions continues to glare at her until Letha gives up and trudges back to her table.

Letha peeks over at Kim again, frustrated.

The movie camera makes a clicking sound, and the film strip in the reel unwinds from the camera and is threaded through a tiny slit in the floor.

INT. EDITING ROOM - DAY

The film strip moves through a small gap in the ceiling of another room.

The strip is wound onto another reel that is mounted on a table below.

YOUNG LETHA ( 9 - pale) sits at the table, manually winding the film strip onto the mounted reel.

When she's done she snips the film strip with some scissors, takes the reel off the mount, and replaces it with a new reel.

She grabs a pen, and writes the day's date and time on the first film reel's casing.

She winds the film strip that is now dangling from the ceiling onto the new reel until the strip is taut again.

Young Letha then takes the first reel, and places it in a projector that is mounted on a shelf, pointing into an adjoining screening room through a little window.

Young Letha hits the play button, and watches the moving, but now-silent, images on a screen in an empty screening room.

The events unfold differently this time:

Letha heads over to Kim's table and very politely greets them.

Kim immediately stands up, and points her plastic fork in Letha's face.

Greta stands up, and gets Kim to sit back down.

Greta then proceeds to verbally abuse Letha, until she cowers back to her table to the sympathetic gaze of other inmates.

Letha glances over at the table and is met by Greta's glare. 
The image of Greta crackles and jolts until GRETA 2 - a copy of the Greta that remains on the screen - walks out of the screen and into the screening room.

Young Letha watches as Greta 2 takes in her surroundings, confused.

Young Letha heads into the screening room through a concealed door, and down a spiral staircase.

INT. SCREENING ROOM - DAY

Greta 2 watches, dazed, as the rest of the film strip comes to an end.

Young Letha stands behind her.

$$
\text { Hello. }
$$$$
\text { YOUNG LETHA }
$$

Greta 2 turns to young Letha, wide-eyed.

Young Letha stares at Greta 2, and speaks in bored tone.

YOUNG LETHA

Welcome to my mind.

Eh?

GRETA 2

Young Letha inspects Greta 2 to make sure everything is in order. She lifts Greta 2's arms up, and pats her legs down.

She then flips Greta 2's hands over, and compares her palms to her own - Greta 2 has no lines or creases on her palms, but Young Letha does.

Young Letha, satisfied, retrieves a folder from a cabinet in the corner.

Young Letha proceeds to talk as if she's an authoritative adult who has done this thousands of times before.

$$
\begin{gathered}
\text { YOUNG LETHA } \\
\text { Can you please tell me your name? }
\end{gathered}
$$

GRETA 2

Greta.

Young Letha looks down at the folder, and nods.

$$
\text { YOUNG LETHA }
$$

And your relationship to me is?

GRETA 2

Uh. I'm in prison with you? 
Good.

Young Letha hands over the folder to Greta 2.

YOUNG LETHA

The answers to most of your

questions will be in this induction

pack.

Greta 2 stares at the folder which has "Letha Beverly Maven: Induction" written on the cover.

YOUNG LETHA

But the main thing you need to know

is that you're a projection of the

Greta that's out there. This

doesn't mean that you're not real,

just that you have a different

function here.

Young Letha goes over to a door that has an "exit" sign above it.

YOUNG LETHA

You're going to be taken to my

consciousness where you'll be given

a housekeeping job to do during the

day, and - if requested - you'll

travel into my dreams at night.

Greta 2 is struggling to process all this.

YOUNG LETHA

If I forget about you, or you're no

longer significant in my present-

day life, your body will

disintegrate but you'll continue to

exist in my long-term memory until

you're triggered again, which might

not ever happen.

Young Letha pauses to see if Greta 2 is following.

YOUNG LETHA

Does that all make sense?

Greta 2 hesitates.

Kind of? GRETA 2

Young Letha puts her hand on the door handle to the exit.

YOUNG LETHA

A boat will come for you shortly. 
Young Letha opens the door, which leads to a metal platform that overlooks the raging ocean that surrounds them.

EXT. SCREENING ROOM/ PLATFORM - DAY

Greta 2 nervously steps outside, and takes in her surroundings.

It is now evident that they are standing on a lighthouse in the middle of the roaring sea.

The light at the top of the building flashes as it scans the ocean.

Young Letha, remains inside, and yells to be heard over the crashing waves.

\section{YOUNG LETHA \\ Thank you for your service!}

Young Letha closes the door.

INT. SCREENING ROOM - DAY

Young Letha heads back through the concealed door into the editing room.

INT. EDITING ROOM - DAY

Young Letha takes the film reel out of the projector.

She sends the reel down a chute - labelled "short-term memory" - that is in the floor under her table.

Young Letha takes a seat at her table again. The film strip that descends from the ceiling has become slack as more film has been fed through from the other room.

Young Letha winds the film strip onto the reel until it becomes taut.

She snips the strip with her scissors - starting the whole process over again.

\section{TITLES .}

EXT. MANSION - DAY

It's early morning, and everything is quiet and still. 
A FEW UNIFORMED GUARDS are sitting on the roof, keeping their eye on the tree - which is currently being restrained by tight wire meshing - as well as surveying the surrounding property.

INT. MANSION, BEDCHAMBER - DAY

Willow 2 is asleep on her pile of blankets.

Kim 2 is asleep on the bed.

Sara has slipped out of bed and quietly heads for the door.

Kim 2 rolls over and wakes up to see Sara about to leave.

KIM 2

Where you going?

Sara turns to Kim 2 .

SARA

Julian asked me to help him with

something .

Kim 2 scowls at Sara, pressing her for the truth.

SARA

I'm not just gonna sit around and

let Letha control me.

Kim 2 shakes her head, angry.

KIM 2

What about your baby?

Sara stares down at her pregnant belly, listless.

SARA

This isn't my baby.

Sara slips through the door.

INT. MANSION, CORRIDOR - DAY

Sara heads down the corridor towards a hatch in the floor.

She tentatively pulls it open, and heads down some stairs into:

INT. MANSION, BASEMENT - DAY

The basement is set up in preparation for an emergency - but only for a select few. 
Julian 2 is standing in a doorway that leads to a corridor that is filled with water, like an indoor river. He is stocking a rowboat that bobs on the surface of the water with a rucksack of supplies and a lantern.

$$
\text { Hi. }
$$

SARA

Julian 2 turns to Sara, and gives her an appreciative smile.

From his pocket, Julian 2 pulls out a little slip of paper with numbers written on it. He hands it to Sara.

\section{JULIAN 2}

This is the memory I need you to bring back.

Sara takes the bit of paper from Julian 2, and tucks it away in her pocket.

She walks over to the doorway, leans over, and stares into the cavernous corridor.

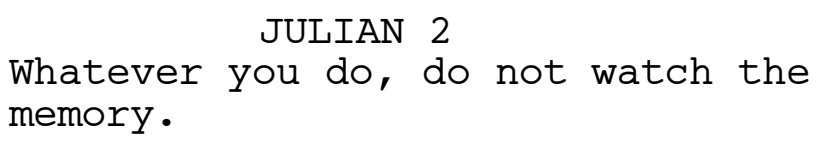

Julian 2 eyes Sara sternly. Sara turns back to Julian 2 .

$$
\text { JULIAN } 2
$$

I know that me saying "don't watch

it" is going to make you want to

watch it but I swear on my life

it's in your best interest that you

don't.

Sara struggles to contain her curiosity.

SARA

Why can't you just tell me what's on it?

JULIAN 2

You wouldn't believe me.

Sara is dubious. Julian 2 sighs.

JULIAN 2

You saw how my so called family

couldn't have cared less that Letha

tried to have me hanged, right?

Sara, feeling sorry for Julian 2, nods.

JULIAN 2

It's because of that memory. 
Sara lets her curiosity go for now. She makes her way onto the boat.

Julian 2 assists her.

Sara takes her seat on the boat, when Julian 2 has a thought.

$$
\text { JULIAN } 2
$$

You might come across some people

or things that are trying to escape

Letha's long-term memories to get

back into her consciousness. Just

ignore them. They can't get in

unless Letha remembers them.

Sara's curiosity is piqued again.

Sara looks down the corridor, into the echoey darkness ahead of her.

$$
\text { OK. }
$$$$
\text { SARA }
$$

Julian 2 leans over and unties the rope.

Sara grips the paddle, and Julian 2 gives the boat a little push with his foot.

Be careful.

Sara waves to him, then paddles into the darkness.

INT. PRISON, COMMON ROOM - DAY

Letha is writing a letter at a table in the corner, when Megan and Beadie sit down with her.

They tentatively eye Letha and her letter.

\section{MEGAN}

What you writing?

Letha doesn't look up from her paper.

Beadie and Megan frown at each other. Beadie leans over and snatches the letter from Letha.

\section{Excuse me!}

Letha tries to get it back, but Beadie successfully dodges her.

Beadie reads the letter allowed so Megan can hear it. 
BEADIE

"Dear Kim, I thought that if I wrote down my thoughts then you could read it in your own time, and come to a more educated conclusion about me. Heaven knows that I am not perfect, but I am not what you and the rest of the world - seem to think I am. Nothing in this life is black and white."

Megan frowns, confused.

LETHA

Please give it back.

Beadie continues reading.

BEADIE

"When I was a little girl I stole

another girl's doll, and then I

watched on as a boy took the blame

for it..."

Beadie trails off, and groans.

BEADIE

This is boring.

Beadie hands the letter back to Letha.

MEGAN

Why're you trying to trick her into thinking your innocent?

Letha laughs, caught off guard.

LETHA

Trick her? I'm not trying to trick her!

Megan looks really weirded out.

MEGAN

You don't have to lie to us.

Letha looks Megan dead in the eyes.

LETHA

I'm not lying to you, dear.

Megan and Beadie share a glance.

MEGAN

What about what you said to me yesterday? 
Letha smiles, and shakes her head. She acts like Megan is the crazy one.

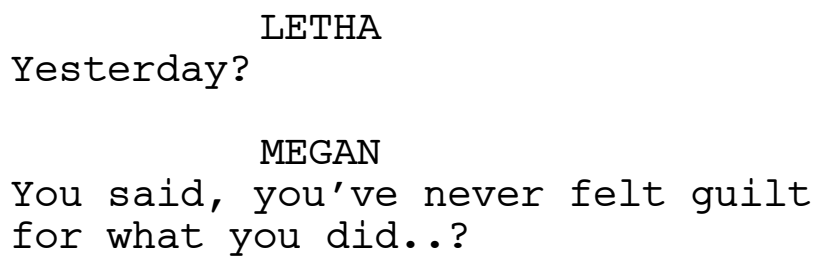

Letha shakes her head again, still smiling like a goon.

\section{LETHA}

Why would I say I never felt guilt

for something I didn't do?

Letha eyes Megan, tensely, still smiling.

$$
\begin{gathered}
\text { MEGAN } \\
\text { OK....Sure...Maybe I imagined it. }
\end{gathered}
$$

Letha nods, agreeing.

Megan sinks back in her seat, disturbed.

LETHA

My son has found a way to prove my innocence.

Megan looks like she understands Letha's strange behaviour now.

Letha goes back to writing the letter.

INT. MANSION, LIBRARY - DAY

Letha 2, apprehensive and paranoid, is sitting at a table that she has placed next to the window.

Charlotte 2 and Emily 2 are playing with a doll's house - a miniature replica of the mansion.

Julian 2 appears from the adjoining room, looking flustered.

Letha 2 turns to look at him, but seems uninterested in his obvious stress.

Julian 2 goes to a shelf and pulls down a book with "Childhood: Vol 2" on it.

Julian 2 is about to head back into the adjoining room when Letha 2 speaks.

LETHA 2

Everything OK?

Julian 2 stops in front of the door to the adjoining room. 
JULIAN 2

Yeah. Think so. Just been having an

influx of requests for stuff that

I'm not so familiar with.

Letha 2 nods, uneasy.

Julian 2 disappears into the room.

INT. MANSION, SCREENING ROOM - DAY

A small screening room - like the one in the light house.

There is a wooden bench in front of the screen. To the side

is a small shelf of tatty and overplayed film reels.

Above the shelf is a neon sign that flashes the word "playground" then "adrenaline rush".

Julian 2 takes a seat, and flicks through the scrapbook

looking for something. He lands on a page that has a

photograph of a 1950s playground.

Julian 2 looks relieved. He reads the date of the photograph, then retrieves a film reel from the shelf that matches the date.

He puts the film reel into the projector, and takes a seat.

He takes care not to look directly at the screen.

On the Screen:

The image flickers then clears up to reveal the $1950 \mathrm{~s}$ playground from the photograph. There is no sound and it is all from Letha's perspective:

YOUNG LETHA (from the beginning), dangling from a high bar, watches as a LITTLE GIRL joyfully walks her plastic doll through a puddle.

The little girl becomes distracted by a bird in the distance. She drops the doll and runs towards the bird to scare it into flight.

Young Letha eyes the doll greedily, and sees that the girl is still distracted, so makes her way down the ladder.

Young Letha creeps over to the doll, picks it up, shoves it in her coat pocket, and runs to a merry-go-round on the other side of the playground.

Young Letha watches as the girl returns to where she left her doll, and becomes panicked and sad when it's not there. She surveys the area to no avail, then sees a young boy on a swing watching her. 
The girl marches over to him and yells at him. The boy bursts into tears.

Young Letha grins.

INT. CORRIDOR RIVER - DAY

Sara, apprehensive, continues to row the boat down the cavernous corridor, but it gets so dark that she has to stop. She lights her lantern and places it at the other end of the boat.

The lantern illuminates a small area ahead of her, just enough to confirm that she's not going to crash into anything.

The light also reveals the litter and debris floating in the water - strange objects like an old, sodden teddy bear, various items of clothes, soggy books etc.

Sara continues on, fighting through her fear, when she hears movement in the water ahead.

Sara rows towards the sound, until she can tell it's right up ahead. She shuffles to the other side of the boat, and holds the lantern out ahead of her, looking for the source.

She doesn't see anything, until there's some movement in the water again, and she sees a MAN's head burst through the surface of water gasping for air.

Sara jumps in fright, and tries to paddle around him. But the man's hand, which is missing several fingers, grips onto the side of the boat.

Sara clearly recognises this hand from her story. She stands up, alarmed, and struggles to keep her balance.

The OLD MAN (60s) pulls himself onto the boat, and flops onto the deck. Sara can now see his gaunt and ghostly face. He puffs and wheezes.

$$
\begin{gathered}
\text { SARA } \\
\text { I can't help you. }
\end{gathered}
$$

The old man - remaining on his back - takes a moment to recover.

SARA

Please get off my boat.

The old man has a realisation, and sits up. He darts his head around. 
Sara looks confused.

SARA

No I'm not.

The old man looks bamboozled.

OLD MAN

You're planning on going into her memories?

Sara nods.

OLD MAN

You'll have to turn around - only

Lethas can get in there.

The Old Man tries to take the paddle off Sara, but she resists.

SARA

She's given me access.

The Old Man's eyes widen with hope.

OLD MAN

Really? How? Doesn't matter.

The old Man sits down.

OLD MAN

I can help you.

Sara is taken aback.

SARA

I don't need your help.

The Old Man laughs.

OLD MAN
Have you ever been into her
memories before?

Sara shakes her head.

OLD MAN

There're things you need to know

before going in there, or else

you'll get lost and never come out

again.

Sara is concerned, but tries her best to hide it.

OLD MAN

I'll tell you everything I know, if

you promise to get Letha to

remember me. 
The Old Man pulls out a film reel wrapped in a cloth.

OLD MAN

All you have to do is play this

memory of me in her consciousness.

Sara shifts her auspicious glance to the Old Man.

SARA

What's on it?

The Old Man glances down, sombre.

OLD MAN

Me giving her a gift on her

birthday.

Sara eyes him suspiciously.

OLD MAN

It's the only memory of me she has.

The old Man gets a bit choked up, and takes a moment to contain himself.

OLD MAN

I died when Letha was young, so she doesn't ever think of me.

Sara isn't very moved by his heart-felt words.

OLD MAN

I just want to hold her in my arms

again and tell her that I love her.

You're lying.

The Old Man is taken aback.

SARA

Who are you to Letha? Really?

The Old Man looks at Sara - a loving sadness his eyes.

Grandpa Maven.

OLD MAN

Sara goes silent as she surveys Grandpa Maven's missing fingers.

OLD MAN

I just want her to remember me.

Sara nods.

OK. 
Grandpa Maven looks immensely happy and relived.

Oh, thank you!

GRANDPA MAVEN

SARA

But can you drop the act?

Grandpa Maven looks confused.

GRANDPA MAVEN

I don't know what you're talking about.

SARA

Yeah, sure.

Grandpa Maven turns to the direction that they're heading.

GRANDPA MAVEN

Keep paddling for a little while, and we'll soon reach the tricky bit where the river opens out into the ocean. After that I'll tell you all you need to know.

EXT. PRISON, EXERCISE COURTYARD - DAY

Several inmates are scattered around the courtyard, getting their daily exercise.

Letha is sitting at a bench, watching as Kim runs laps around the small, uneven track. Letha grips her letter to Kim.

Letha makes her way over to the track and waits for Kim to approach her.

Kim speeds up when she sees Letha, forcing Letha to speak quickly and increasingly loudly so that Kim hears her as she passes by .

LETHA

I know you won't talk to me, but

will you at LEAST READ MY LETTER?

Kim continues running. Letha waits for her to finish another lap.

When Kim approaches again, Letha holds out the letter for Kim to take.

Kim dodges Letha and her letter, and goes for another lap.

Letha deflates, and grows irritated again. She waits for Kim to return, and this time she stands on the track.

Kim sprints towards her, but Letha doesn't move. 
Kim stops right before slamming into Letha.

KIM

Babies can't defend themselves!

Kim rips the letter out of Letha's hand and tears it into

little pieces and drops them on the ground. She stomps on the pieces until they're trodden and muddy.

Kim continues running.

Letha steps off the track and watches as other inmates run around the track and trample on her letter.

Letha heads back to her bench, furious.

She sees the group of hardened inmates, who walked out on her storytelling session, and marches over to them.

$$
\text { Hello, ladies. }
$$

LETHA

The inmates look at her with disdain.

LETHA

You don't happen to be in the

business of hurting people, do you?

The inmates' eyes all go wide.

LETHA

Or know of anyone who is?

The GROUP LEADER of the inmates, eyes her up and down.

$$
\begin{aligned}
& \text { GROUP LEADER } \\
& \text { Doesn't come for free. } \\
& \text { LETHA } \\
& \text { I'll give you anything. } \\
& \text { GROUP LEADER } \\
& \text { Not give. DO. }
\end{aligned}
$$

Letha processes what this means.

\section{LETHA}

Yes. Of course. I'll do anything.

The group leader makes a hand gesture to a BURLY WOMAN across the quad. She heads over. Letha glances excitedly at Kim who continues to run around the track. 
INT. CORRIDOR RIVER - DAY

Sara and Grandpa Maven continue to row in silence down the corridor, when the current starts to pick up, and an opening of light can be seen in the distance.

GRANDPA MAVEN

Here we go.

Grandpa Maven turns to see how far away they are.

GRANDPA MAVEN

I'll get out to push the boat, you

stay on board to paddle, OK?

Sara nods, apprehensive.

Grandpa Maven pulls himself up.

GRANDPA MAVEN

Promise you won't take off without

me.

SARA

Promise you won't take off without me.

Grandpa Maven nods. Sara nods back.

Grandpa Maven lets himself off. He grips on to the back of the boat with his finger-less hands.

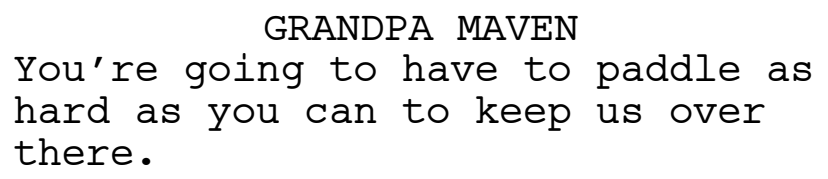

Grandpa Maven nods his head towards the other side of the corridor.

Sara readies her paddle.

They are about to reach a point where one wall vanishes, revealing a rugged ocean that aggressively pushes and pulls at the water in the corridor.

Sara looks up ahead to where the corridor resumes as normal.

Go!

GRANDPA MAVEN

Sara paddles as hard as she can, while Grandpa Maven grips the back of the boat and kicks his feet in the water with all his might.

They manage to move towards the remaining wall of the corridor, where the current isn't so rough. 
But soon their energy wanes and they're dragged back towards the ocean.

They both strain and sweat to keep on track, but it's a losing battle.

Grandpa Maven loses his grip.

Sara stops paddling, and grabs hold of him, but he screams at her.

\section{GRANDPA MAVEN}

Keep paddling!

Sara lets go of his hand, and continues paddling. But it makes no difference.

Grandpa Maven is practically drowning.

The boat is ripped out of the corridor and into the ocean.

$$
\text { I can't control it! }
$$

Grandpa Maven is left behind, treading water. He holds his film reel above his head to keep it from getting too drenched.

\section{Abandon ship!}

GRANDPA MAVEN

Sara, panicked, floats further out to sea. She is about to jump off the boat, when she sees the lighthouse in the distance.

She stares at it, mesmerised by the flashing light.

GRANDPA MAVEN

The further you go, the further it

is to come back!

Sara takes out the bit of paper with Julian's reel number written on it, and tucks it into her hair to keep it dry.

She lowers herself out of the boat, and - keeping her head above the water - thrashes her way through the water towards Grandpa Maven.

The boat floats away.

Grandpa Maven and Sara slowly make their way through the water together until they're finally back in the corridor.

They walks along the wall, until the current dies down and without a thought they hug each other until it gets weird and they peel away from each other. 
GRANDPA MAVEN

Sorry about your boat.

SARA

Not my boat.

Grandpa Maven frowns at her.

GRANDPA MAVEN

Whose was it?

SARA

What's that lighthouse?

Grandpa Maven eyes Sara, suspicious. Then starts wading through the water, away from the current and into the darkness.

\section{GRANDPA MAVEN}

Don't you remember it from when you arrived?

SARA

I arrived in a desert.

Grandpa Maven raises his eyebrows, understanding.

Sara follows after him - pushing her way through the water. GRANDPA MAVEN

It's where Letha's projections are formed and recorded.

Sara is fascinated.

\section{GRANDPA MAVEN}

They say there's a room in there

that reveals everything that Letha

is seeing and hearing in the

present, but I've never believed it

myself.

Sara can't believe what she's hearing. She turns and looks back at the mouth of the ocean that's now nearly out of sight.

\section{GRANDPA MAVEN}

So, if it wasn't your boat, does

that mean you're doing all this for

someone else?

Sara prises her eyes away from the gap in the wall, and continues on behind Grandpa Maven.

SARA

I'm not doing anything for anyone

else. 
Grandpa Maven looks puzzled by this.

GRANDPA MAVEN

Well that's good. Cos it would be stupid to risk your life for someone else...

Sara looks alarmed by this.

Grandpa Maven holds out his missing fingers.

GRANDPA MAVEN

The only way to escape a memory if

you get trapped in one, is to cause

yourself so much pain that it

breaks the reality of the memory.

Sara falls silent, petrified.

GRANDPA MAVEN

Are you prepared to do that for whatever it is you're doing?

Grandpa Maven surveys Sara as she considers this.

Sara looks at Grandpa Maven again, with returned determination.

Yes.

SARA

Grandpa Maven gives her the side eye, clearly not convinced. GRANDPA MAVEN

You're a lot like Letha, you know?

Sara, annoyed by this comment, doesn't respond.

GRANDPA MAVEN

Letha was always quiet and reserved

on the outside, but I could tell

there was a lot going on upstairs.

SARA

I'm not like her.

GRANDPA MAVEN

Nothing wrong with being like her.

SARA

She killed her own babies.

$\mathrm{Hm}$.

GRANDPA MAVEN

Sara frowns at this non-committal response. 


\section{GRANDPA MAVEN}

I suppose it's natural that her

story character is like her.

SARA

I'm not like her.

GRANDPA MAVEN

I'm just saying - as much as we

try, we can't escape those who

shape us.

Sara turns her head in protest, and the two wade through the water in silence for a while.

Grandpa Maven sighs.

\section{GRANDPA MAVEN}

The main thing is, you have to act

like you know what you're doing and

where you're going.

Sara looks confused.

\section{GRANDPA MAVEN}

In her long-term memories, I mean.

Once you're in there, don't talk to

anyone...

Sara listens, coyly.

INT. PRISON, COMMON ROOM - DAY

Letha enters the common room, and is annoyed to see that the TV is back in working order. A couple of people are watching a trashy talk show.

Letha takes a seat in the corner where a LANKY WOMAN is sitting.

\section{Maven?}

\section{LANKY WOMAN}

Letha nods.

\section{LANKY WOMAN}

Package'll drop tonight during

dinner, out on the field, sixth

fence post along.

The Lanky Woman eyes Letha to see if she understands. Letha nods again.

My payment? 


\section{LANKY WOMAN}

It'll happen around the same time.

Letha smiles, pleased.

The Lanky Woman stands up and leaves. The inmates who were watching the TV follow after her, leaving the TV on.

Letha goes over to the TV, and unplugs it.

She drops the plug on the floor and stomps on the inlet so that its prongs are mangled.

Letha exits, satisfied.

INT. CORRIDOR/ RIVER BANK - NIGHT

Sara and Grandpa Maven reach a river bank that leads up to the entrance to a high-rise office building.

Grandpa Maven steers the boat to the side of the building, and Sara gazes up at it with apprehension.

Grandpa Maven looks worried for her.

$$
\begin{aligned}
& \text { GRANDPA MAVEN } \\
& \text { Recite the directions again? } \\
& \text { SARA } \\
& \text { I think I've got it, thanks. } \\
& \text { GRANDPA MAVEN } \\
& \text { OK, but - what do you do to make } \\
& \text { sure you don't get sucked into a } \\
& \text { memory? }
\end{aligned}
$$

Sara speaks reluctantly.

\section{SARA}

Sit on the side of the screening room, and don't look into the people's eyes.

Grandpa Maven nods. He pulls out his film reel from his coat, and holds it out for Sara to take.

$$
\text { GRANDPA MAVEN }
$$

Can you take this with you, in

case, god forbid, something happens

and I don't see you again?

Sara stares at the reel, ominous.

GRANDPA MAVEN

You'll be fine. But, you know, nothing's certain. 
Sara takes the real, and tucks it into her dress.

Grandpa Maven smiles, grateful.

Sara smiles, reluctantly.

SARA
Thanks for your help.

Sara heads towards the entrance to the building.

EXT. LONG-TERM MEMORIES BUILDING - NIGHT

Sara stands in front of the sliding doors, searching for a way to open them. She notices a sensor to the side of the door.

Sara turns her hand over, revealing the web of creases and wrinkles on her palm.

Fighting through her nerves, Sara stands in front of the sensor and places her trembling hand on it.

Sara waits for the doors to open, but nothing happens.

She takes her hand off and tries her other hand. She holds it there longer this time, and eventually it makes a beeping sound and the doors slide open.

Sara breathes a sigh of relief, and heads into the building.

INT. LONG-TERM MEMORIES BUILDING, LOBBY - NIGHT

Sara steps into a reception area where a TEENAGED LETHA (1960s hairstyle and clothes) is sitting behind a desk.

Sara walks past her, straight ahead, into the main building. Excuse me?

TEENAGED LETHA

Sara ignores her, and marches through several rows of shelves filled with film reels. A sign reveals that these are "New Arrivals".

The Teenaged Letha stands up to catch a better look of Sara. She picks up a phone on her desk, and makes a call - a suspicious look on her face.

Sara marches on. Different VERSIONS OF LETHA (at various ages, and dressed in clothes form different eras) are scattered throughout the building - browsing, stocking the shelves, and keeping watch.

To the left of Sara is a sign that points to "Screening Rooms". Sara looks to her left and sees a row of booths. 
Swirling in and out of these booths are ghostly figures, trying but failing to escape.

Sara turns to the right and heads for the elevator.

INT. LONG-TERM MEMORIES BUILDING, ELEVATOR - NIGHT

Sara steps into the elevator with a MIDDLE-AGED LETHA who stares at her suspiciously.

Sara nods to her, and tries to act like she's been here before.

She scans the panel of buttons which are organised by every year that Letha has been alive - from "1944" to "2018".

But there is also a button for a level labelled "Restricted" and another button for "Screening Rooms/ New Arrivals" on the ground floor.

Sara feels Middle-aged Letha's eyes on her, so she subtly slips out Julian's piece of paper which has "7.7.1970/00:28" written on it.

Sara hits the button for "1970".

The elevator creaks and groans as it ascends. It stops at "1951", and the middle-aged Letha steps out.

Sara sees that the room is fairly empty, with about ten shelves of reels in total.

The doors close, and Sara breathes a sigh of relief at being alone again.

The elevator takes her to the floor for "1970".

INT. LONG-TERM MEMORIES, FLOOR “1970” - NIGHT

Sara steps off the elevator, and finds herself in a dark room with rows and rows of dusty shelves.

As Sara makes her way into the room, a harsh fluorescent light comes on.

Sara sees that the rows of shelves are organised by weeks of the year. She makes her way towards week 28 .

Sara scans the film reels for the one that matches Julian's date and time, and when she eventually finds it she takes the film reel off the shelf, and tucks it away in her coat pocket.

She scans the film reels that surround the now-missing reel, and sees that there are memories marked with times very close to when Julian's takes place. 
Sara takes down the film reel that comes immediately before Julian's memory, and the one that comes immediately after it.

She heads back to the elevator, and is relieved to see that it is empty.

INT. LONG-TERM MEMORIES, ELEVATOR - NIGHT

Sara is about to hit the button for the ground floor where the screening room is, but instead hits the button for the restricted floor.

The light for this button starts flashing and then an alarm goes off.

Sara panics as the doors start to close. She quickly slips through the doors and pops back out into the floor for 1970s.

INT. LONG-TERM MEMORIES, FLOOR “1970” - NIGHT

The alarm continues to blare as Sara watches the elevator close then shoot up to the "restricted" level.

INT. LONG-TERM MEMORIES, STAIRCASE - NIGHT

Sara makes her way down the 26 flights of stairs.

INT. LONG-TERM MEMORIES, GROUND FLOOR - NIGHT

Sara, puffed, pokes her head through the door into the ground floor.

She sees that, despite the alarm still going off, the coast is clear.

She creeps over to the screening booths, and makes her way into the only vacant one.

INT. LONG-TERM MEMORIES, SCREENING BOOTH - NIGHT

Sara catches a glimpse of the teenaged Letha coming towards her.

\section{That's her!}

$$
\text { TEENAGED LETHA }
$$

Sara slams the door closed and locks it. The teenaged Letha knocks on the door, but Sara ignores it.

Sara takes in her surroundings. She's in a small room with a projector that points to a screen, and a chair that is positioned in the centre of the room. 
Sara moves the chair to the side, then places the first film reel into the projector - the memory that comes right before Julian's.

The knocking finally stops.

$$
\begin{aligned}
& \text { TEENAGED LETHA (O.S.) } \\
& \text { I'm notifying security. }
\end{aligned}
$$

Sara hits play, takes a seat, and watches the screen making sure not to make eye contact with the people in the memory.

On the screen, from the perspective of Letha:

Letha (26) is in bed with Julian (24) - they're laughing and talking together when something unknown to us causes them to stop.

They sit together, not talking, and clearly struggle to ignore whatever it is.

Letha buries herself in her pillow. She peeks from under her pillow, and sees Julian making his way out of the room.

Letha closes her eyes. The film ends, and the screen goes black.

Sara goes over to the projector, and takes the film reel out. She replaces it with the memory that comes straight after Julian's. She hits play and takes a seat.

On the screen, from the perspective of Letha:

Letha (26) is sitting in the corner of a different room, cradling a baby whose body is limp and a ghastly blue colour.

Letha rocks back and forth, until the screen goes black again.

Sara stares at the black screen, astounded. She pulls out Julian's film reel from her coat, and replaces it with the one in the projector.

She takes a moment, before hitting play and taking a seat.

On the screen, from the perspective of Letha:

Letha (26) wakes with a jolt in her bedroom, and looks as if she hears something in another room.

She sees that Julian isn't in bed.

She sits up in her bed, and listens for more sound.

Letha jumps out of bed, and heads into the hallway. 
Letha opens the door to another room, and pokes her head in Julian (19) is standing over a baby's crib with a pillow in his hands.

He turns to Letha with a grave expression. They remain frozen in this position for stretch of time.

Sara is astonished.

There is a loud knock on the door to Sara's booth. SECURITY LETHA (O.S.)

This is security, you need to unlock this door right now.

Sara stands up, panicked.

SECURITY LETHA (O.S.)

If you don't open up, we will force entry.

Sara turns back to the screen.

There is a loud bang against the door as security begin forcing entry.

Sara, panicked, stares straight into Julian's eyes on the screen, and is enveloped by the memory.

INT. HALLWAY, 1963 - NIGHT

Sara stands in the corner of the bedroom. She watches as Letha enters, pushes past Julian, and goes to the baby's crib.

Sara stands beside Letha and they both stare down at the baby - its skin is a sickly blue, and it's not moving or

breathing. Letha can barely look at it.

Tears fill Letha's eyes. She turns, and charges at Julian, shoving him into the wall.

Julian pushes Letha back. Sara, outraged, steps in between them to try to protect Letha, but she's shoved out into the hallway.

Before Sara can see what happens next she hears the sound of a door clicking open behind her, and everything goes dark.

Sara gasps and puts her arms out to feel her surroundings she's now in a small space.

A light hanging from the ceiling is flicked on to reveal that she's now in a 1960 s bathroom. 
INT. 1960S BATHROOM - NIGHT

The person who has flicked the light on is JOAN (20s - pale, faint and ghostly).

Stunned, Sara scans the bathroom and notices another WOMAN slumped in the bath tub, dead and decaying.

Joan sits back down on the tiled floor, exhausted from the effort it took to turn the light on.

$$
\text { Did Julian send you? }
$$

INT. PRISON, DINING HALL - NIGHT

The dining hall is a hub of movement and noise.

The hardened inmates are all sitting at a table together, subtly eyeing up Kim in the distance.

Megan takes a seat at her table, and notices Letha's seat is empty.

EXT. PRISON, COURTYARD - NIGHT

The courtyard is dark and deserted.

Letha appears in the shadows, and scurries over the track towards the fence line.

Letha waits, tensely, at the edge of a security light's beam.

She hear's a bird cooing from somewhere on the other side of the fence. Letha tries to see where the bird is, but fails.

\section{Kaaaa! Kaaaa!}

LETHA

Letha waits for a response. When the bird coos again, she smiles - pleased with herself.

\section{Kaaaa! Kaaaa!}

LETHA

The bird coos in return, but it is cut short by the sound of footsteps.

Letha tenses.

Maven?

MALE VOICE (O.S.)

LETHA

Yes. 
SEAN (16 - wearing a school uniform) appears on the other side of the fence.

LETHA

$$
\text { Aren't you a bit young? }
$$

Sean looks offended.

$$
\text { Ain't you a bit old? }
$$

Letha looks offended.

Sean puts his hand in his pocket, and pulls out several clusters of cigarettes tied together with red string.

\section{SEAN}

Boss said to drop 'em in the sanitary bin in the second stall of the third block toilets.

Letha nods.

$$
\begin{gathered}
\text { SEAN } \\
\text { Don't be taking none for yourself. }
\end{gathered}
$$

Letha laughs.

$$
\text { I don't smoke. }
$$

Sean looks at her like she's an idiot.

SEAN

Smoke's is like money and if you

get caught stealing you'll get

beat.

At that, Sean turns on his heals and trudges away.

Letha shoves the cigarettes into her underwear, and heads back towards the prison.

EXT. PRISON, BACK ENTRANCE - NIGHT

Letha pushes on the door to open it, but it's locked.

\section{Shoot.}

LETHA

Letha tries the door again. It doesn't budge.

Letha spins around, trying to see another way into the prison.

She tries the door one more time, to no avail, before scouring the perimeter of the building. 
INT. 1960S BATHROOM - NIGHT

Sara is trying to find a way out of the bathroom, to no avail.

JOAN
I've been searching for years.

Sara keeps looking.

$$
\text { JOAN }
$$

And she searched before me.

Joan gestures to the dead body in the bath.

$$
\text { We're the same. }
$$

Sara discovers a loose tile, and lifts it up. A hole has been dug out, but stops short of any hope.

SARA

I'm not the same as you.

Sara keeps looking for a different way out.

JOAN

You're from a story, right?

Sara stops looking, but keeps her back turned to Joan.

JOAN

So was I. And so was she. We were told by Julian that we were unique and special, and we both convinced ourselves that we could somehow hurt Letha and find a way out.

Sara turns to Joan.

JOAN
Letha goes through cycles of
wanting to understand things. She
creates people like us to break
through for her, but then when we
start to get close she locks us
away.

Sara is struggling to process all this.

SARA

Did Julian know this would happen?

Joan pauses, then nods. 


\section{JOAN}

I reckon he must know that it's

hard-wired into us to find an

escape, so he tricks us into

thinking we're helping him when

really he's just trying to get rid

of us.

Sara shakes her head, not quite understanding or believing.

SARA

He could have just executed me like

he did the little boy the other

day.

Sara sees that the mirror above the bathroom sink opens up.

She lunges over to it and opens it - but it's empty.

Sara closes it again, but inspects the glass of the mirror.

SARA

You saw that memory, right?

Sara looks at Joan in the reflection of the mirror. Joan nods.

SARA

It makes sense that he'd want that destroyed.

Joan sighs.

JOAN

I think maybe he wanted us to see

it before he got rid of us. Like

he's proud of it or something.

Sara looks at herself in the mirror, she's not convinced by Joan's explanation.

\section{JOAN}

That Letha's let him get away with

it.

Sara takes in a deep breath, then plunges her fist into the mirror.

Joan jumps in fight.

JOAN

What the hell?

Sara picks up one of the shards of glass, and turns to Joan. 


\section{SARA}

If I get Letha to remember that

memory, maybe the truth will set us

free.

Sara takes a seat on the tiled floor.

She takes another deep breath, then starts sawing her pinky finger off with the shard of glass.

Sara screams in pain, but persists. Joan looks like she might faint.

EXT. PRISON, WALL - NIGHT

Letha creeps alongside a wall, looking for a way in, when she hears a kerfuffle going on in the distance.

Letha power-walks to the end of the wall, and peers around the corner.

EXT. PRISON, RUBBISH BIN ZONE - NIGHT

A couple of BUFF INMATES have Kim pinned to the concrete pavement and are beating her to a pulp. She screams in pain.

\section{KIM \\ Please! Stop!}

A MALE GUARD is standing at a distance, watching it all go down while keeping an eye out.

In the distance, Letha watches on - a sinister grin on her face.

The buff inmates stop pummelling Kim. They both head off with the guard.

Kim is left on her own, sprawled on the ground, bleeding and groggy.

Letha steps forward to follow after the other three, but Kim sees her in the corner of her bloodied eye.

$$
\begin{aligned}
& \text { KIM } \\
& \text { (nearly inaudible) } \\
& \text { Lefa. }
\end{aligned}
$$

Letha stops and turns to Kim, who is unable to move anything but her eyeballs, which plead at Letha.

$$
\text { Help. KIM }
$$

Letha smiles at her condescendingly. She nudges Kim with her foot, getting a smudge of blood on her shoe in the process. 


\section{LETHA}

This is what it feels like when

people refuse to listen to you.

Letha walks away, eerily calm.

EXT. PRISON, DINING HALL - NIGHT

Letha power-walks to catch up to the buff inmates and the guard who sneak back into the prison through the dining hall.

As the door is about to close after them, Letha bounds over and stops the door right before it closes.

Letha slips back inside.

INT. PRISON, DINING HALL - NIGHT

The dining hall is empty, except for a few INMATES who have stayed behind to clean up.

Letha lingers by the door for a moment, before striding across the dining hall, head down and inconspicuous.

INT. PRISON, CORRIDOR - NIGHT

Letha hurries down the corridor.

INT. PRISON, CORRIDOR (OUTSIDE BATHROOM) - NIGHT

Letha heads into the bathroom.

INT. PRISON, BATHROOM - NIGHT

Letha goes into the second stall and deposits the cigarettes into the sanitary bin.

INT. 1960S BATHROOM - NIGHT

Sara is writing in pain, clutching her blood-drenched hand. The tip of her pinky lies on the floor.

JOAN

Why did you do that?

Sara looks faint.

SARA

Someone told me it'd get me out of here. 
JOAN

I'm telling you, you're not as

special as you think.

Sara looks like she's starting to believe Joan.

JOAN
There's no point to trying.

INT. PRISON, COMMON ROOM - NIGHT

The common room is filled with inmates all taking turns at looking at the smashed TV plug.

Letha enters, and sidles up to Megan who looks at her with a strange expression.

$$
\begin{gathered}
\text { MEGAN } \\
\text { Where've you been? }
\end{gathered}
$$

Letha is off-puttingly jovial.

$$
\text { Nosy parker! }
$$

Megan grimaces at her cheery demeanour. Her face falls completely flat when she notices the smudge of blood on her shoe.

LETHA

What's going on?

MEGAN

Someone busted the TV plug .

LETHA

Oh, deary me!

Letha goes over to the crowd that has formed around the TV, and Letha ushers them away. The inmates are so taken aback by her tenacity that they don't protest.

LETHA

Sit down. Sit down.

Some inmates sit down, and others remaining standing bemused.

Letha inspects the plug.

LETHA

That's no good at all, is it? I

guess I'll have to tell you some

more of my story.

The inmates groan and slump in their seats, which aggravates Letha greatly. 
Megan tenses at Letha's obvious frustration.

LETHA

Now, now, children. I have

something to say, and you owe it to

me to listen.

SASHA

Why? You're just some crazy bitch

who did her own babies in.

Other people laugh and agree.

Letha's eyes pop, and her fists clench. She subtly swings the TV cord back and forth.

Megan steps in.

\section{MEGAN}

Maybe we should hear Letha out?

Everyone turns to Megan, grimacing and frowning. Megan eyes them all, tensely.

\section{MEGAN}

Where did you finish, Letha? Sara

was in the tower..?

Letha smiles at Megan, immensely pleased.

\section{LETHA}

Yes. Sara was told by Thomas, her young guard, that the town was preparing to execute her that night.

Some of the inmates slump further into their seats, and others stand up and leave. Letha ignores them, and loses herself in her story.

\section{LETHA}

Sara was a smart and determined girl, and wasn't about to give up easily. She looked into Thomas' eyes and saw fear and sadness in him. She wondered why someone so young would have been given the job of keeping watch of a potential witch. So she asked him...

INT. MEDIEVAL TOWER - NIGHT

Sara is back in the tower - bound in ropes. She jiggles the rope around her wrist so it's less uncomfortable and we see that her pinky is still attached. 
While Sara can't control her movements or what she's saying, there's a look of relief in her eyes.

She glances at THOMAS (13 - wearing an impressive livery which barely conceals how young and scrawny he is).

\section{SARA}

Why have you been put in charge of keeping watch over me? Aren't they worried I could do witchcraft on you?

Thomas clearly hadn't thought of this, but tries to conceal the fear it induces.

$$
\text { I asked to be here. }
$$

Sara doesn't quite follow.

THOMAS

I wanted to prove to my father that

I'm responsible.

SARA

Who's your father?

Thomas puffs his chest, proudly.

\section{THOMAS}

He's the leader now, since the old

one died. I guess we have you to

thank for that.

Sara smiles, taking all this information in.

\section{SARA}

He must be proud of you.

Sara notices that Thomas winces before responding.

THOMAS

I don't need him to be proud of me.

Sara nods again, pretending to understand.

The two fall silent for a moment.

SARA

Would you mind if I told my baby a story?

Thomas frowns at her. 
SARA

It's just that I usually tell my

baby a story every night, and since

this will be our last night I want

to make it special.

This fills Thomas with guilt, but tries hard not to show it.

Fine.

THOMAS

Thomas leans back in his chair.

Sara puts her hand on her pregnant belly, and clears her throat.

SARA

This story takes place in the distant future. 700 years from

now... And it's about a a boy. A

young man, whose father was rather

cruel to him.

Sara looks to see if this has sparked Thomas' interest. Thomas sees through her, and plays hard-to-get.

THOMAS

My dad's not cruel to me.

SARA

This story isn't about you.

Thomas folds his arms.

\section{SARA}

The young man's father wanted him

to be a powerful and strong member

of society, and was disappointed to

see that his son was full of

womanly emotions and ideas. So he did everything he could to toughen

him up. The young man had been

forced by his father to cut down a

tree with a tiny axe, he'd wrestled

with wild animals, and now his

father wanted him to dig a hole.

Thomas laughs.

A hole?

THOMAS

Sara is pleased to see Thomas has been listening.

SARA

Yes. The father told his son to dig

a hole. 
Sara marks a dot in the dust on the floor.

EXT. FIELD - DAY

A deep hole pierces the earth. A rope dangles down the side of it.

The SON (played by Thomas) is at the bottom of the whole digging deeper and deeper.

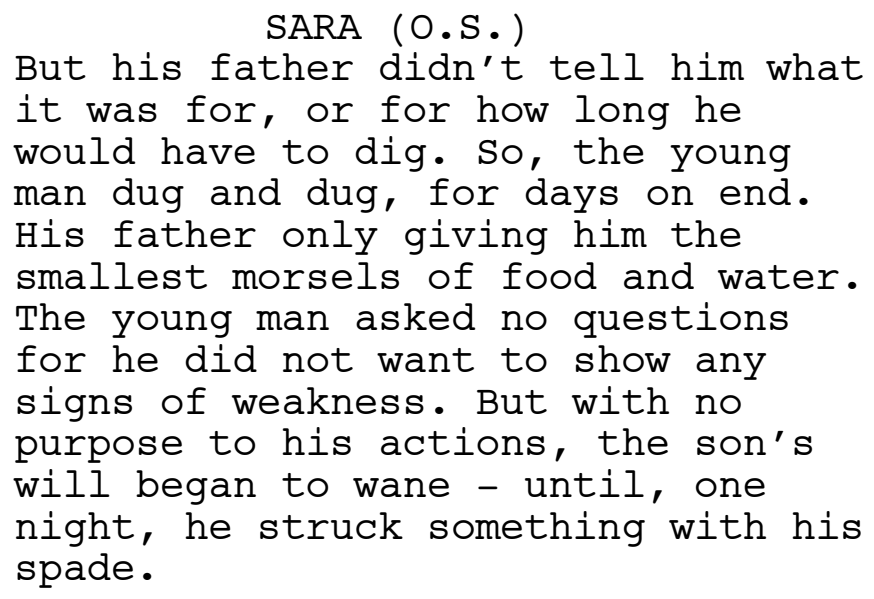

Thomas hits something in the ground with his shovel, and stops digging as someone screams from below.

$$
\text { SARA (O.S.) }
$$

Was this why his father had told

him to dig, he wondered. Or was he going crazy?

Thomas uncovers what looks like a tree root.

Thomas yanks it out of the ground, lifting a layer of soil and revealing a labyrinth of underground tunnels.

Screams and voices can be heard crying out from below.

$$
\text { Hello? }
$$

$$
\text { THOMAS }
$$

$$
\begin{aligned}
& \text { UNDERGROUND VOICE (O.S.) } \\
& \text { Don't let the light in! }
\end{aligned}
$$

Thomas, astonished, yells to the sky.

\section{Father!}

$$
\text { THOMAS }
$$

Thomas is so far down the hole that there's no way anyone above ground would hear him.

I said stop the light!

$$
\text { UNDERGROUND VOICE (O.S.) }
$$


Thomas lowers himself into the underground tunnel.

INT. UNDERGROUND - DAY

The underground is exactly the same as what appeared in Letha's dream.

Thomas squeezes himself through some tight spots and eventually lands in an opening where several NAKED FIGURES surround him.

Thomas instantly regrets his decision to investigate. He tries to scramble back up, but some of the FIGURES hold him down and another one blocks the hole that is letting in a thin stream of light.

\author{
FIGURE \\ What are you doing down here? \\ THOMAS \\ Nothing. I just. I was digging \\ and... well....Do you live down here?
}

The figures all look baffled.

FIGURE 2

You were never told?

Thomas shakes his head, utterly confused.

The figures all look horrified, that he really doesn't know.

FIGURE

What's the point of it, if they

don't even know their origins?

FIGURE 3

They still have to live up there

and battle the elements.

FIGURE 2

And it means we continue to have

nothing to do with them.

THOMAS

What are you talking about?

An ELDERLY FIGURE who has been quietly surveying Thomas, steps in.

ELDERLY MAN

We live off peace and love down

here.

Thomas inspects his surroundings, and notices how calm and peaceful it is; no reason for anxiety or fear. He relaxes. 
ELDERLY MAN

Centuries ago we banished some of

our people when the started

exhibiting evil behaviour - hunger

and thirst and lust - disrupting

the order of things. They were

forced to live above ground with

the horrors of light, seasons, and

open air.

Figure 2 pins Thomas down with his foot. Thomas tries to struggle free.

FIGURE 2

You're a descendent of those evil

people.

Thomas frowns, confused.

THOMAS

But doesn't that also mean I'm a

descendent of you guys?

The figures are outraged by this claim. Figure 3 forces his foot down harder on Thomas' chest.

Thomas becomes defensive.

THOMAS

Wasn't it evil of you to banish

your own people rather than try to

help them change?

The Elderly Figure is impressed with this logic, but remains silent.

The other figures are outraged by his accusation.

FIGURE

They weren't capable of changing.

Thomas is regretful of angering the figures.

THOMAS

I'm capable of changing.

The figures sense that Thomas is getting ideas.

FIGURE 3

You don't belong here.

The figures, except for the elderly man, all descend upon him.

THOMAS

I can learn how to live off love and peace. 
They pick him up, and shove him back up the tunnel.

THOMAS

I don't want to go back!

Thomas tries to struggle free, but it's no use.

Wait.

ELDERLY FIGURE

The other figures stop moving but continue to hold Thomas in a tight grip.

ELDERLY FIGURE

Who do you live with up there?

THOMAS

It's just me and my father.

The Elderly Figure pauses, thinking.

Is he kind to you?

\section{ELDERLY FIGURE}

Thomas doesn't respond. The Elderly Figure nods, knowingly.

ELDERLY FIGURE

If you kill your father, we'll let

you live with us down here.

Thomas goes limp. The others can't believe what they're hearing.

What?

FIGURE 2

Thomas shakes his head.

$$
\begin{aligned}
& \text { THOMAS } \\
& \text { I can't do that. } \\
& \text { ELDERLY FIGURE } \\
& \text { Very well. }
\end{aligned}
$$

The Elderly Figure nods to the others who then push Thomas up into the tunnel.

Thomas looks back down at the figures, longingly, but makes his way back up the tunnel.

EXT. FIELD - NIGHT

Thomas, dirty and exhausted, pops through the ground and pulls himself back into the hole.

He clambers up the rope. 
INT. HOUSE - NIGHT

Thomas trudges into the house where his FATHER (the fingerless man) is eating dinner, alone.

The father looks at Thomas, disdainfully.

FATHER

Given up already?

Thomas takes a seat.

I found them.

$$
\text { THOMAS }
$$

Found what?

FATHER

Thomas is confused.

THOMAS

The people who live down there.

Thomas' father shakes his head, irritated at his son's nonsense.

THOMAS

You don't know about them?

FATHER

You trying something with me?

THOMAS

Why were you making me dig, then?

Thomas's father laughs.

Because I can.

FATHER

Thomas lets this sink in.

THOMAS

Are you proud of me?

The Father frowns at him in disgust.

FATHER

What good would pride do?

Thomas is filled with rage, but he tries to contain it. THOMAS

Would you care if anything happened to me?

The Father stares at his son like he's lost his mind. 
Thomas stands up, furious.

THOMAS

Answer my question.

The Father speaks resentfully.

FATHER

It would be harder to get things

done around here with only my pair

of hands.

The Father displays his finglerless hands.

Thomas can't contain his rage any longer. He lunges for his father, and strangles him with his bare hands.

His father falls back in his chair, and smacks his head on the ground. His body goes limp, and a stream of blood trickles from the back of his head.

Thomas releases his grip from his father's neck.

Father?

THOMAS

His father doesn't respond. Thomas's whole body trembles, but he manages to pull himself up and scramble outside.

EXT. FIELD - NIGHT

Thomas makes his way down the rope, into the hole again.

But when he gets to the bottom, the entry into the underground is not there.

Thomas digs deeper into the hole, but there's only more soil. Thomas drops the shovel, and falls to his knees.

He looks up at the starry sky above, delirious.

The faint sound of a door knock can be heard, but goes unnoticed by Thomas whose world becomes pixilated.

Everything in the world, including Thomas, disintegrates into sand.

INT. TOWER - NIGHT

Sara has stopped telling her story because someone is pounding their fist on the door.

Thomas, who has clearly been engaged and slightly shaken by Sara's story, gets up and opens it. 
TWO BURLY MEN are standing in the doorway.

BURLY MAN

They're ready for her.

Sara panics, and pleads with Thomas.

SARA

You could do something.

Thomas closes his eyes, to block Sara out.

\section{THOMAS}

She's all yours.

SARA

Don't you want to hear the rest of

the story?

The burly men step inside. They grab Sara, roughly, and carry her out.

Thomas!

SARA

INT. TOWER, STAIRCASE - NIGHT

Sara struggles in the men's grasp.

$$
\text { Help! }
$$

SARA

Thomas follows after them

EXT. GALLOWS - NIGHT

Several TOWNSPEOPLE have gathered in front of the gallows.

The fingerless man is standing on the platform, ready for Sara.

The burly men carry Sara towards the gallows. Sara continues to squirm and struggle.

Thomas joins the townspeople.

\section{Stop!}

SARA

At that, the world becomes pixilated and everyone around her including the burly men - collapses to the ground.

Sara remains conscious as she watches her surroundings disintegrate into sand, and she finds herself in the vast desert again - astonished and relieved. 
Sara starts making her way across the desert, when a voice stops her.

$$
\text { Help. THOMAS (O.S.) }
$$

Sara turns and sees that Thomas is lying on the ground, halfdisintegrated.

Sara sees that Thomas isn't able to move. She turns, and walks away.

INT. PRISON, COMMON ROOM - NIGHT

The remaining inmates are staring at Letha, confused and concerned.

Letha has stopped talking, and is frozen on the spot. Her hand is resting on her hip, and her fingers are pressed against a bulge in waist-line of her pants from which a piece of red string dangles down.

Letha scurries out of the room to the dismayed laughs of the inmates.

$$
\begin{gathered}
\text { SASHA } \\
\text { (to Megan) } \\
\text { Why'd you make us listen? }
\end{gathered}
$$

Megan speaks seriously.

\section{MEGAN}

She's not right in the head.

DES

You scared of a little old lady?

The others laugh. Megan is not amused.

$$
\text { Yes. I am. }
$$

$$
\text { MEGAN }
$$

The others laugh even more.

\section{MEGAN}

Did you not hear her story just now?

INT. PRISON, CORRIDOR - NIGHT

Letha hurries down the corridor. 
INT. PRISON, BATHROOM - NIGHT

Letha bursts into the second stall. She rips the lid off the sanitary bin, but - to her horror - sees that there's nothing inside.

Letha pulls out the cluster of cigarettes - that she forgot to dispose of - from her pants, and stares at them with a grave expression.

LETHA

(genuine terror)

You've done it now, you nincompoop!

EXT. DESERT - NIGHT

Sara watches the faceless figures form and disintegrate into sand around her.

She sees the mansion in the distance and runs towards it.

EXT. MANSION, FRONT ENTRANCE - NIGHT

Sara slows down as she approaches the mansion's front entrance.

She's about to head in when she notices the guards on the roof, and walks around to the back entrance instead.

EXT. MANSION, BACK ENTRANCE - NIGHT

Sara creeps around the back when she notices a plume of smoke rising from in the back garden.

Curious, Sara follows the smoke.

EXT. MANSION, BACK GARDEN - NIGHT

Sara pushes her way through the overgrown weeds and shrubs until she finds herself at a small bonfire that Julian 2 is sitting in front of, clutching onto a film real.

Julian 2 notices Sara, and stands up - startled.

JULIAN 2

How'd you escape?

Sara notices the film reel in his hands.

SARA

Is that your memory?

Julian 2 looks down at the reel. 


\section{JULIAN 2}

They sent them to us after you

disappeared.

Sara notices that the three other film reels are at his feet one of them is Grandpa Maven's, still wrapped in his cloth.

$$
\text { We have to play it. }
$$

Sara lunges for the reel, but Julian 2 side-steps her.

$$
\text { JULIAN } 2
$$

What you saw in this memory didn't really happen.

Sara frowns, offended that he would think she'd fall for that.

SARA

I don't care about what you did.

Sara tries to grab it from him again, but they just end up in a tussle.

$$
\text { JULIAN } 2
$$

It's a false memory. Letha made it

up to protect herself from the

truth.

Sara keeps trying to yank the reel out of hands.

SARA

So why haven't you destroyed it yet?

JULIAN 2

I'm going to, I'm just wary of what

might happen once it's gone.

Sara, curious, stops tussling with him, and steps backwards.

SARA

What might happen?

\section{JULIAN 2}

She'll have one less security

blanket.

Sara takes a moment to process this, and has a change of heart.

Sara finally yanks the reel free from Julian 2 and throws it into the fire.

Julian 2 and Sara watch it burn. 
INT. 1960S BATHROOM - NIGHT

Joan remains slumped on the bathroom floor.

Joan sits up a little when she smells, then sees, a thick smoke coming from under the doorway.

$$
\begin{aligned}
& \text { SARA (O.S.) } \\
& \text { What's going on? }
\end{aligned}
$$

Across from Joan, Sara - sitting in her smeared blood from the disembodied pinky that sits next to her - stares at the door, groggily.

The door starts to melt.

$$
\text { I don't know. }
$$

Joan, panicked, stands up, but there's not much they can do as the entire bathroom melts around them.

Joan and Sara scream as they catch fire.

EXT. MANSION, BACK GARDEN - NIGHT

Julian 2 is looking extremely alarmed as Sara screams out in pain.

$$
\begin{aligned}
& \text { JULIAN } 2 \\
& \text { What's happening? }
\end{aligned}
$$

Sara drops to the ground and rolls around as if to put out the flames.

Once the film reel is completely destroyed, Sara finally stops writhing.

$$
\begin{aligned}
& \text { JULIAN } 2 \\
& \text { Are you OK? }
\end{aligned}
$$

She stares up at the dark sky, incensed.

SARA

I was still in there.

Julian 2 frowns, confused. He stares at the now-mangled film reel in the fire.

Sara pulls herself up.

\section{SARA}

She's gonna pay.

Sara grabs Grandpa Maven's film reel from the pile, and heads back towards the mansion. 
Julian 2, panicked, follows after her.

INT. PRISON, BATHROOM - NIGHT

Letha is sitting on the toilet, staring at the cigarettes, when she hears stomping footsteps approaching accompanied by outraged mutterings.

$$
\begin{aligned}
& \text { FEMALE VOICE (O.S.) } \\
& \text { Fucking bitch is gonna get it. }
\end{aligned}
$$

Letha panics. She drops the cigarettes into the toilet, bursts out of the stall, glances around, sees a large rubbish bin.

$$
\text { FEMALE VOICE (O.S.) }
$$

Fucking bitch is gonna get it.

As the footsteps get closer, Letha takes the lid of the bin, and steps inside.

She's placing the lid over her head, when a YOUNG GUARD enters the bathroom and flicks the light on.

The young guard has headphones on and was clearly listening, and singing along, to a song. She frowns when she sees Letha putting the lid over her head.

$$
\text { What the? }
$$

The young guard cautiously steps over to the bin.

$$
\text { Hello...? YOUNG GUARD }
$$

Letha doesn't respond.

The young guard takes the lid off the bin and sees Letha peering up at her.

$$
\begin{aligned}
& \text { YOUNG GUARD } \\
& \text { What are you doing? }
\end{aligned}
$$

Letha pretends to be utterly lost and confused. She glances around.

LETHA

I don't know! Where am I? What's going on?

The young guard steps back, alarmed.

$$
\text { Help. }
$$

LETHA

The young guard speaks into her walkie-talkie. 
INT. MANSION, LIBRARY - NIGHT

Sara storms through the dark library. Julian 2 is not far behind.

$$
\begin{aligned}
& \text { JULIAN } 2 \\
& \text { (whispering) } \\
& \text { What's on it?! }
\end{aligned}
$$

Sara heads into the adjoining screening room.

INT. MANSION, SCREENING ROOM - NIGHT

Sara quickly glances around the room.

She presses a button on the projector, which lights up the screen.

Julian 2 enters, and continues to whisper urgently.

$$
\begin{gathered}
\text { JULIAN } 2 \\
\text { Please don't do anything rash. }
\end{gathered}
$$

Sara glares at Julian 2 .

$$
\text { I'm not her plaything! }
$$

Julian 2 gestures for her to be quiet.

Sara places Grandpa Maven's film reel in the projector. She hits the play button and takes a seat.

On the screen:

TEENAGED LETHA is lying in bed, blissfully listening to a record, when her bedroom door opens.

GRANDPA MAVEN (now with all his fingers) walks into the room with a large birthday gift in his arms.

Sara frowns, extremely disheartened.

$$
\text { SARA }
$$

He wasn't lying.

Teenaged Letha sits up and looks excitedly at the present in Grandpa Maven's arms. He hands the gift over.

Teenaged Letha rips it open, revealing a doll's house - the miniature replica of the mansion.

Teenaged Letha smiles at Grandpa Maven - clearly not too pleased with the gift.

Sara leans in, curious. 
Grandpa Maven takes a set on Teenaged Letha's bed, and eyes her greedily.

Teenaged Letha cowers on her bed.

Grandpa Maven shuffles closer to Teenaged Letha, who now has no room to escape.

Sara is grinning now.

$$
\text { SARA }
$$

Yes!

Julian 2 looks at her like she's a maniac.

JULIAN 2

I'm stopping it.

Julian 2 makes a move for the projector, but Sara lunges on him - tackling him to the ground.

SARA
She needs to suffer.

Julian 2 can't believe what he's hearing.

JULIAN 2

You're worse than she is!

Sara, still on top of Julian 2, continues watching the screen.

Grandpa Maven places his hand on Teenaged Letha's thigh, then leans into her.

The image of Grandpa Maven crackles and jolts.

Julian 2 tries to throw Sara off him, to no avail.

Grandpa Maven walks out of the screen and into the screening room. Grandpa Maven inspects his fingers, pleased.

He sees Sara and Julian 2 on the floor.

$$
\begin{aligned}
& \text { GRANDPA MAVEN } \\
& \text { Oh, hello again. }
\end{aligned}
$$

Grandpa Maven steps around Sara and Julian 2 and heads out of he screening room.

INT. MANSION, LIBRARY - NIGHT

Grandpa Maven calmly makes his way through library •

Sara and Julian 2 step out of the screening room, unsure what to do. 
INT. MANSION, LETHA'S BEDCHAMBER - NIGHT

Letha 2 is resting on her bed.

Grandpa Maven pushes the door to Letha 2's bedchamber open, and stands in the doorway.

When Letha 2 sees him, she cowers, and the room begins to shake as if an earthquake is striking.

EXT. MANSION - NIGHT

The guards on the roof are asleep, but wake to the vigorous shaking.

The tree is growing again - it makes cracking sounds as its branches expand and turn black.

The tree envelops the entire mansion.

INT. MANSION - NIGHT

The mansion is completely dark.

The sound of objects smashing and crashing is interjected with terrified screams.

INT. PRISON, BATHROOM - NIGHT

Letha has fallen out of the rubbish bin, and is curled up in foetal position on the floor in a catatonic state.

An OLDER GUARD has turned up and is at Letha's side.

The older guard, confused, turns to the younger guard who keeps her distance from Letha.

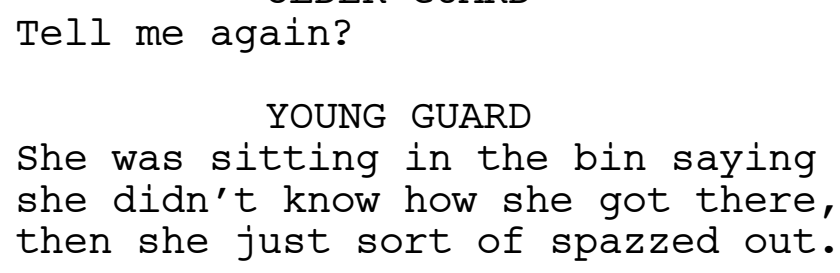

The Older Guard turns back to Letha.

$$
\text { OLDER GUARD }
$$

Can you hear me, Letha?

Letha is completely still, except for her flickering eyelids.

\section{End of Episode.}




\section{UNTOLD AND CHAOS}

Something that became immediately apparent when attempting to write serial dramas that resembled chaotic systems was the difference between observing the structures in a serial through a lens of chaos and writing to the structures of a serial through through that same lens. In a way, it was easier to discuss chaos in terms of shows that already existed-to fit my understanding of chaos around them in an objective way-than having to actually construct narratives that resembled chaotic systems in a way that didn't sacrifice the story. In other words, it was one thing to acknowledge the similarities between chaos and the narrative structures of serial dramas, but it was a completely different thing to implement them in my own writing. This was particularly evident throughout the process of writing my first TV project, Untold.

The idea for Untold originated from my desire to produce a loose adaptation of 1001 Nights, and use the various fractal-like properties of TV serials to write narratives embedded within narratives. I faced many challenges when attempting to achieve this, especially that I was prioritising structure over the story. I liked the novelty and 'craftiness' of the embedded narratives more than I liked the narratives themselves. For instance, the first draft had the serial open with Sara waking up in a medieval town, surrounded by a dead family that she doesn't know, and subsequently being accused of being a witch and causing plague in the town. Sara is sent to a tower to await her execution but is watched over by a young boy to whom she starts telling a story in an attempt to delay her death. This story branched off into a new story, and then another new story-eventually leading to Sara's discovery that she can escape into her own stories because she herself is just a character in someone else's story. I initially thought I had a sustainable narrative, but as I ventured further and further into the embedded stories, I realised all I had was a structure. In a workshop where my first draft of the pilot episode was read by fellow PhD students, the serial was received with a sense of trepidation and doubt. Their concern was that it wasn't possible to write a consistently compelling and engaging story for TV that was constantly shifting into new stories with different worlds and characters. This point fits into the argument I make in my 
critical component-that once a serial sets up its own bounds of logic and tone, it can't stray too far from those bounds or else an audience will tune out.

What I learnt as I continued writing and rewriting this project was that it wasn't necessarily the embedded narratives themselves that were the problem, but rather the way in which I was using them. I needed the embedded narratives to embody the wants and needs and stakes of the main characters as a way to tie them all together so that they functioned individually as well as contributing to an ongoing narrative for the serial as a whole. I needed to implement the hybrid-form of open-ended and closed-off narratives-that I examined in relation to strange attractors - to greater effect. After taking a break from the project for a year, I reapproached it with both a new perspective and a new objective.

My new approach was to start with the outermost frame that shows the person telling Sara's story, rather than beginning somewhere in the middle as I had been (with Sara waking up in the plague-ridden town). The reason I had started in middle was that I liked the mystery as Sara became conscious of herself as a fictional character, but it turned out this mystery was actually just causing confusion. It was coming across as pure randomness, rather than randomness by design. So, I introduced Letha from the very outset of the narrative and showed her being sent to prison for something she claims she didn't do, in an attempt to provide the necessary connective tissue between each of the frames that would subsequently unfold. Introducing Letha's story allowed me to have the stories unravel in an embedded and disconnected way, but also to show them to be part of the same thing. That is, everything that happens in the serial can be boiled down to Letha's journey as she transforms from a kindly old grandmother to a psychopathic woman who is capable of anything.

In this sense, the serial is fractal in that Letha's and Sara's storylines are self-similar; Letha is breaking free from her false persona that she's manufactured for herself while Sara is trying to break free from Letha. While their motivations and trajectories are slightly different, their method for achieving their goals is essentially the same (because they are essentially the same person)-to work through and reorganise everything that has been muddied or suppressed in Letha's mind. Beyond these two threads, the fractal nature of the serial also 
comes through in the embedded stories that Letha tells. That is, Letha's story about Sara reflects her own situation in which she has been locked up for something she initially claims she didn't do. But as Letha starts to unveil the truth about who she really is, the embedded stories change to reflect this. For example, in Episode Two Letha tells the story of Sara telling a story to her guard about a young boy who kills his father after a group of underground dwellers tell him to, only for him to realise that the underground dwellers never existed. I included this story to reflect the way in which Letha is cautiously starting to reveal who she really is and what she's capable of, which in turn reflects, and impacts, Sara's journey as her desperation to escape grows. In the next episode Letha tells the story of Sara telling the story of the young boy listening to a story told about an old man who was led to believe that the events in his life were all a dream even though they were actually real. Again, this shift in the embedded stories marks a shift in Letha and reveals how she views the world and other people as easily manipulated, but also reflects Sara's uncertainty about what is real and what isn't.

By utilising the fractal-like nature of serial drama narratives, the stories that Letha tells also function in a way that reflects sensitive dependence on initial conditions. As Letha changes and grows as a character, the types of stories she tells also change, resulting in a cascading effect that reflects Letha's process of going from claiming that she is innocent to admitting her guilt and unleashing her true, psychopathic personality. In turn, there is a dynamical relationship between the narrative components of this serial: the stories that Letha tells impact the environment in which Sara finds herself in Letha's mind, which in turn impacts the kinds of memories and emotions that Sara uncovers in Letha's mind, which further impacts the kinds of stories Letha tells.

Since a large part of Untold takes place inside Letha's mind-essentially encompassing the lifetime of a 70-year old woman-there has always been, and will continue to be, the risk that the serial will be convoluted and confusing. Indeed, the process of writing the serial has been convoluted and confusing. But, using chaos as a lens through which to write this serial has illuminated the need for simplicity and containment in order for complexity and creativity to flourish. My process of navigating the messiness and confusion by introducing simplicity is reflected in the physical space and geography of Letha's mind which continues 
to transform with every new draft. In an early version of the script, Letha's mind was as a contained theatre where a random group of people performed Letha's memories and dreams. In a subsequent draft Letha's mind became a ramshackle tent in the middle of the desert where everything in Letha's life existed all at once in a disordered mess. And now, in the current draft, Letha's mind is mansion that is run by the most significant people in Letha's life and that has pre-established rules and structures that its residents are all aware of. Turning Letha's mind into a space that is more ordered, familiar and grounded in something that resembles reality has allowed me to explore and augment the aspects of Letha's psyche that are more abstract and surreal. Writing Untold with chaos in mind has demonstrated that order and simplicity in serial dramas enhance, enrich and are necessary for the narrative form's complexity. The next serial I am presenting is Bad News, which also demonstrates this relationship between simplicity and complexity but in a different way. 


\section{BAD NEWS}

\section{Written by}

Alison McLachlan 



\section{BAD NEWS}

6 x 30-minute comedy series

A motley group of emotionally damaged individuals who have no business meddling in others' affairs sets up a company that breaks bad news for, and to, other people.

Elliot is crippled by neuroses, Trina desperately wants to feel needed, Jayesh turns everything into a joke, Barry has serious anger issues, and Leone relishes other people's misery.

Together the five of them form Bad News: a service that allows people to avoid their personal problems.

But are the members of Bad News really bettering their lives and helping others? Or are they using the business to avoid their own personal problems and creating havoc in the process?

As the business grows and expands, the five are faced with an interweaving thread of cases that pushes them to their limits - sometimes bringing the group closer together, and other times tearing them apart.

How far are people willing to go in order to offload their emotional burdens? 

CHARACTERS 



\section{ELLIOT}

28.

Elliot Mercer doesn't want too much out of life. Just a solid relationship, a cruisy job, and a comfortable bed.

But when his boyfriend breaks up with him for being a boring pushover, Elliot becomes determined to prove to the world (mostly his ex-boyfriend) that he can be unpredictable and adventurous. Elliot steals his ex-boyfriend's idea for a break-up business and sets up Bad News with his newly-found odd-ball friends. But Elliot quickly realises the business is going to push him further out of his comfort zone than he might be prepared for.

Elliot's shyness, neuroses, and inability to confront things makes him the worst possible person to break bad news. But as the Bad News business expands, Elliot must learn how to face issues head-on, or risk losing the business and his dignity.

Once he's committed to the business, there's no way he wants to go back to his old life as the boring, timid Elliot he once was.

\section{TRINA}

17.

Trina Wong would probably be described by others as "a bit much". She likes people, but people don't tend to like her.

As a result, Trina is a lonely and insecure person who constantly finds herself caught between the immaturity of a child and the bossiness of an adult. She does everything she can to make her younger sisters' lives as stress-free as possible following their parents' divorce, but as a result she is actually making things more stressful, and it becomes clear that it's really her that is struggling with the divorce.

When Trina finds out about Elliot's Bad News business, she does everything she can to be a part of it - seeing it as an opportunity to be needed by others, make friends, and get closer to Jayesh.

While Trina's pluckiness is a big asset for Bad News, her clingy and needy tendencies aren't appreciated quite so much. When pushed to her limits, Trina has to deal with the fact that she can't force and manipulate people into needing her. 


\section{JAYESH}

21.

Jayesh Bansal is friendly, charming and outgoing and if he just stopped with his relentless lame jokes, people might take him more seriously.

A few years ago when Jayesh accidentally killed his grandfather with an undercooked chicken, Jayesh abandoned his unforgiving family and moved in with the reclusive Leone and Barry-who understand his pain because they've each accidentally killed someone as well.

But this reclusive lifestyle doesn't suit Jayesh's gregarious personality so when a chance encounter with Elliot leads to the formation of Bad News, Jayesh leaps at the chance to get out in the world again, and to use his sense of humour for good.

Jayesh's confidence, humour, and gullibility often get him into trouble, and in order to truly help the business succeed he must access the sensitive and loving side of himself that underpins his desire to make people laugh.

\section{LEONE}

50.

Leone Miller is motherly, passive aggressive, and a bit crazy. She thrives off other people's drama and tragedy, but she is so unaware of this that thinks she has a genuine gift for empathy and compassion.

Leone has been living with Barry and Jayesh for several years and has been lying to them the whole time. They think that, like them, she has also accidentally killed someone, when she's actually just made the whole thing up in order to be a part of their tragic situation.

Leone joins Bad News with the intention of using her non-existent empathy skills, but really she just wants to meddle in other people's problems and drama.

But when Leone is forced to come clean to the group, she must confront the fact that her thirst for tragedy comes from her inability to relate to people-especially people who are happy. 


\section{BARRY}

65.

Barry Walsh is unapologetically blunt and brazen. Or-as he'd like to phrase it-he's a truth teller.

Barry is initially reluctant to join the Bad News business because, outwardly, he disapproves of the idea but Barry secretly enjoys being in the group and likes to think the business would fall apart without him.

Barry's contribution to the Bad News team is severely limited due to the fact that he's become the living embodiment of a couch potato ever since accidentally killing a little old lady in a house-painting mishap. Naturally, the memory of this accident is sore spot for Barry that, if pressed upon, will unleash his wild temper.

As Barry ventures further away from the couch, he must learn how to control his anger in order to become a truly valued member of Bad News, and to uncover the softer underside of his gruff exterior.

\section{RUSS}

32.

Russ is horrendously arrogant, vindictive and manipulative, but he is also deeply insecure.

For years he's been harping on about his great idea for a break-up business, which he is convinced is going to make him millions. So, when he learns that Elliot, his ex-boyfriend, has stolen his idea and made it even better, Russ sets out to take him and his business down.

While Russ will tell people he's seeking revenge and standing up against the theft of ideas, he is actually worried that Elliot is going to end up much happier and more successful than he ever was when they were in a relationship. And he'll stop at nothing to ensure this doesn't happen-including setting up a rival business called "Breaking Bad News". 



\section{BAD NEWS}

Episode Synopses (1-6) 



\section{BAD NEWS}

\section{Episode One: "Parking Space"}

Just when Elliot Mercer thinks he's finally perfected his effortless lifestyle, his boyfriend breaks up with him and he is ripped out of his comfort zone leading Elliot to set up a Bad News business.

ELLIOT (28) thinks he has the perfect life-a drama free relationship, a comfortable bed, and now a zero-responsibility job.

But after Elliot unwittingly causes a catastrophe that results in a dead body and a dog escaping its leash before his boyfriend, RUSS, sends his cousin, TRINA (17), to break up with Elliot on Russ' behalf, Elliot is forced to reassess his life decisions.

Desperate to win Russ back by proving that he isn't the pushover Russ thinks he is, Elliot does Trina a favour by promising to step in and pretend he has been catfishing JAYESH (21), an online date she is now concerned might be a psychopath.

After being heckled out of a fancy restaurant by the fellow diners for "catfishing" Jayesh, Elliot accepts Jayesh's invitation back to his flat where Elliot connects with Jayesh's freakishly cloistered flatmates-LEONE (51) and BARRY (65)-who reveal that the three of them live together because they have each accidentally killed someone.

When Elliot donates a large sum of money towards Russ' ridiculous Kickstarter campaign for his break-up business, Russ cruelly throws the romantic gesture back in Elliot's face and Elliot vengefully sets up his own business with Jayesh, Leone and Barry that breaks bad news for, and to, other people.

Already down in the dumps about the lack of response to their Bad News ad, and his new job that he now regrets taking, Elliot receives a deranged death threat from Russ who is furious that Elliot has stolen his business idea.

Just as Elliot is about to take the Bad News ad down he receives a call from the woman who was walking the dog that escaped its leash at the start of the episode and needs Bad News to tell the dog's owner that the dog was hit by a car.

Elliot accepts the case. 


\section{BAD NEWS}

\section{Episode Two: "HEALBoI"}

\section{When Bad News bungle their first case, only Trina and her cherished stuffed toy pony can save them.}

Trina thinks she's mature and sophisticated, but when she lashes out at her two little sisters for trying to take her precious toy pony to school she reveals her true childishness.

After Elliot, Jayesh and Leone meet with their first client and are given precise instructions about delivering the dead dog to its owner, they are horrified to discover that the dead dog has been stolen.

After a humiliating display at school, and learning that Russ has edited the Bad News ad so that it looks like it's for a sex trafficking ring, Trina forces herself onto Bad News in an attempt to redeem herself but things only get worse for her when Jayesh doesn't recognise her from her dating profile.

Meanwhile two bumbling cops are alerted about the tampered-with Bad News ad and track the Bad News team, misinterpreting their attempts to replace the dead dog as a sex trafficking operation.

Despite mounting pressures and the fact that she could easily solve Bad News' problem, Trina withholds her toy pony from the Bad News team while continuing to help them in their ridiculous and fruitless attempts to produce a fake dead dog.

But when Trina sees that her sisters are better off without her and her mollycoddling ways, she realises how immature she really is and finally offers her toy pony over to the baffled Bad News team.

Bad News successfully deliver the fake dead dog but are roped into attending a funeral for the dog where the truth is revealed in a mortifying fashion and the police barge in to break up what they think is a child sex trafficking ring.

While the Bad News team are left wondering if their business will ever recover from their bungled first case, Trina says a creepy-but heartfelt-final goodbye to her toy pony, and Russ hijacks the Bad News business and receives a call from a new client.

A fraudulent spiritual healer requests the services of Bad News after he finds his car vandalised and suspects his followers must be on to him. In reality, a young boy has smashed his car out of anger after his friendly neighbourhood dog stopped paying him visits after school. 


\section{BAD NEWS}

\section{Episode Three: “Cabbage”}

\section{Jayesh goes on an ill-advised journey of self-discovery that foils Bad News' plan to seek revenge on Russ.}

After Jayesh receives a message from his estranged family saying his grandmother has died, he avoids the guilt this triggers over his accidental killing of his grandfather by donning a fake persona and going undercover for Bad News in order to sabotage Russ and his case with the fraudulent spiritual healer.

Despite his confidence going in, Jayesh is quickly brainwashed by the wacky teachings of the fake spiritual healer-GEOFFREY GOLDSACK - who convinces Jayesh that all his problems will be solved if he does Geoffrey's housework for him, Mr. Miyagi style.

While Jayesh goes AWOL, the Bad News team are forced to use Leone's dodgy contacts to leak information about Geoffrey to the spiritual healing group, but the information mutates into rumours that spiral out of control and the team wind up having to protect Russ from the bloodthirsty group who now think their spiritual leader has invested the money he stole from them into an evil pharmaceutical company.

When Jayesh realises Geoffrey has been duping him and that he has actually been helping Geoffrey pack up his house so that he can skip town and avoid all his problems, Jayesh lashes out at him and opens up about the guilt he feels over accidentally killing his grandfather.

The Bad News team encourage Jayesh to attend his grandmother's funeral, but when they all get there the team's insistence that his family don't really blame him for his grandfather's death is quashed, prompting Jayesh to make an incredibly inappropriate speech that reads more like a bombed stand-up routine.

While Trina and Jayesh grow closer, despite Trina's dishonesty about her identity, the Bad News team reclaim their business.

Meanwhile, Jayesh reveals his feelings for Trina who-filled with guilt over lying to him about her true identity-pulls away from him.

A man calls up Bad News after he is put in charge of organising a high school reunion for his dying friend-the husband of a member of the spiritual healing group-and who is terrified that his friends are going to find out that he raided their extremely valuable time capsule several years ago. 


\section{BAD NEWS}

\section{Episode Four: "Hearing Aid"}

\section{The Bad News team face a moral conundrum when they realise they can capitalise on Barry's anger issues.}

Barry surprises the Bad News team when he leaves the house for the first time in several years in order to take on the case of telling the group of old high school friends that their buddy raided their valuable time capsule.

But when Barry finds out that the client isn't related to the young girl he accidentally killed as he had thought-and he has therefore left the house for nothing - he becomes uncontrollably angry and delivers the bad news about the time capsule in a blunt, yet remarkably effective, way.

When the Bad News team meet with a new client who has just bought a new hearing aid that means she can now hear how annoying her husband's laugh really is, they agree that the husband's laugh is actually really lovely and none of them have the guts to lie to him.

Impressed with Barry's effectively blunt delivery, the Bad News team intentionally trigger his anger in order to tell the lovely man that his laugh annoys his wife but instead Barry discovers that the wife's hearing aid is faulty and was sold to her by a conman needing to clear his debts-the high school buddy who stole the valuables out of the time capsule.

Enraged, Barry tracks down the conman and is ready to rip him a new one when Leone convinces the Bad News team to help Barry rather than exploit him, prompting Barry to attend a meditation retreat.

When the hearing aid case sparks an inundation of calls from other clients who want to tell their spouses their pet peeves about them, Barry learns that he's terrible at breaking bad news without his anger but he secures his place on the Bad News team when he finally opens up to them about his true feelings about accidentally killing the little old lady.

Hearing Barry open up prompts Leone to drop the massive bombshell that she's been lying to them this whole time about accidentally killing someone.

While the Bad News team grapple with Leone's news, Elliot receives a heart-felt apology from Russ who pleads with Elliot to take him back. 


\section{BAD NEWS}

\section{Episode Five: "UFO"}

\section{While Leone struggles to learn how to be a decent human being, tensions within the Bad News team boil over.}

When Leone showers the Bad News team with kindness and affection, she only exacerbates the tension within the group, forcing Elliot, Jayesh and Barry to travel out of town for a peculiar case in which a woman who said she didn't see a UFO fifty years ago, claims that she did actually see it.

Leone struggles to resist the urge to relish and aggravate the petty marital problems of the continuing influx of spousal pet peeve cases, until Leone is faced with a particular case that hits very close to home and she is unable to cope with an overwhelming feeling that is completely foreign to her - empathy.

Meanwhile, Barry, Elliot and Jayesh become entangled in the small-town feud that erupts after they break the news about the woman's UFO sighting and the woman's ex-best friend comes forward to discount her own claim about seeing the UFO.

Just when Trina has managed to calm Leone down after experiencing empathy for the first time, they see a viral video of Barry, Elliot and Jayesh derailing an interview about the UFO debate by descending into a yelling match in which Jayesh accuses Elliot of being weak and stupid for taking Russ back, and Elliot reveals the truth about Trina's identity.

Upon watching the viral video, Tristan (40s), a mama's boy, proposes to dedicate his life to finding out the truth about the UFO, which vexes his mother into calling Bad News to tell her son that she's been lying to her him his whole life about him having a debilitating illness.

When the three men return from their trip, the Bad News team splinter and Leone is forced to break the news to Tristan alone, but her new-found empathy backfires and she fails to prevent Tristan from intentionally injuring himself while rock-climbing so that he never has to leave his mother.

With the Bad News team still at each other's throats, Leone moves back home with her husband, Jayesh rejects Trina's olive branch, Elliot realises Russ only slept with him to cause tension in the Bad News group, and Barry finds himself in the peculiar position of being the only happy one in the group. 


\section{BAD NEWS}

\section{Episode Six: "Murder"}

\section{As the Bad News team drift further apart, Trina loses control of a case she takes on alone in an attempt to keep the business afloat.}

The members of Bad News are fractured:

Elliot tries to find a rebound but just ends up on dates with guys who are very similar to Russ.

Jayesh badly bombs his first comedy routine.

Leone faces the wrath of her family who can't understand why she abandoned them.

Barry gets into hot water due to his misplaced peace and happiness.

Meanwhile, Trina - the only one who seems to still care about the business-secretly takes on new case by herself in which she has to fire the rock-climbing instructor (who was meant to be supervising Tristan) on behalf of his boss, who just so happens to also be his wife.

Trina gets in way over her head when, in retaliation to being fired, the rock-climbing instructor hires Trina to tell his wife-boss that he's been having an affair with her best friend for years.

Not willing to believe it, the wife-boss hires Trina again to help her raid her friend's apartment for evidence of the affair but they wind up inadvertently embroiling the friend in a murder that occurs at the apartment.

With no one else to turn to, Trina reaches out to Jayesh and the two reconcile and consummate their romantic feelings for one another before setting out to tell the wife-boss' friend that she's now the suspect in a murder case - only Jayesh and Trina accidentally tell the wrong person.

After the wrongly-informed person has a mental breakdown that causes a nation-wide manhunt, Trina and Jayesh force the Bad News team to air their grievances before delving together into the murder case in which, it turns out, the murder victim was caught up in the catastrophe caused by Elliot's illegal parking in the first episode. 


\section{BAD NEWS}

Scripts (Episode One \& Episode Two) 



\section{BAD NEWS}

Episode One:

"Parking Space"

Written by

Alison McLachlan 

EXT. CITY STREET - DAY

A rusty scooter putters down a main street.

The RIDER slows the scooter down when he sees the only free parking space nearby - it is clearly marked as a residential park only. The rider hesitates for a moment, then sneaks into the park.

The rider steps off the scooter, and takes his helmet off he is ELLIOT MERCER ( 28 - lanky and clearly lacking ambition despite the semi-formal clothes he's wearing).

Elliot takes out a crumpled bit of paper from his pocket, kisses it, then ducks into an office building nearby.

A monstrous black four-wheel drive creeps up the street and brakes aggressively when it reaches Elliot's parked scooter. The car has a residential sticker on its windscreen.

INT. CAR/ STREET - DAY

A frazzled MOTHER grips the steering wheel tensely. Her two kids - a GIRL (7) and a BOY (4) - are screaming in the backseat of the car.

GIRL

He's gonna blow!

Shit!

MOTHER

The kids abruptly fall silent. The boy looks sheepish, and the girl scrunches her face up. She pinches her fingers to her nose.

\section{GIRL \\ Shit! Shit! Shit! Shit! Shit!}

The mother is about to lose the plot when she sees a free loading zone up ahead. She accelerates towards it.

EXT. STREET - DAY

The mother leaps out of the car, hurls the boy over her shoulder and ushers the girl out. They rush into their apartment together.

A delivery truck zooms down the road and slows down when the out-of-shape TRUCK DRIVER sees the loading zone is taken.

The truck driver shakes his head, annoyed, then sees a clear driveway further up the street. He manoeuvres into it, stumbles out of the driver's seat and unloads some crates from the back. 
He hauls the crates down the street and into a cafe, sweating and puffing excessively.

A car appears in the driveway, unable to get out because the truck is in its way.

The DRIVER of this car is bawling his eyes out - he's not having a good day, and the truck in his driveway is the final straw. He slams his hand on the horn and sits on it creating an ear-splitting ruckus.

His fury builds the longer he sits there, until he finally breaks. He puts his foot on the accelerator and slams his car into the truck.

The truck driver, still straining with exhaustion, comes out of the cafe and is horrified to see what has happened. He rushes towards the collision, but stops to clutch his chest in pain. The truck driver hobbles towards the car.

The driver is slumped over the steering wheel, a thin stream of blood running down his head.

The truck driver tries to open the door but it's locked. Panicked, he pulls out his phone and dials "111", but he falls to the ground before he's able to speak into the phone.

A PASSERBY walking a dog, runs over to tend to the situation, but in doing so loses grip of the dog's leash.

The dog bounds down the street into the distance.

$$
\begin{aligned}
& \text { PASSERBY } \\
& \text { Tyche, come back! }
\end{aligned}
$$

INT. OFFICE - DAY

Elliot is struggling to balance on a swiss ball while concentrating on what LESTER (50s, dishevelled, also sitting on a swiss ball but with more confidence) is saying from behind his messy desk.

Lester has three framed Warrant of Fitness certificates hanging on the wall, like they're his qualifications.

\section{LESTER}

I'm telling you straight, Elliot it's a terrible job.

Elliot shakes his head, doubtful.

\section{LESTER}

You'll be stuck in a dark basement all day every day, you'll have absolutely no interaction with any other humans, and you'll be doing the most tedious task known to man. 
Elliot smiles, genuinely enthusiastic.

ELLIOT

You just described my dream job.

Lester laughs and shakes his head.

LESTER

You don't need to bullshit me with

your fake enthusiasm.

Elliot's enthusiasm doesn't wane.

ELLIOT

I'm not.

Lester is dubious.

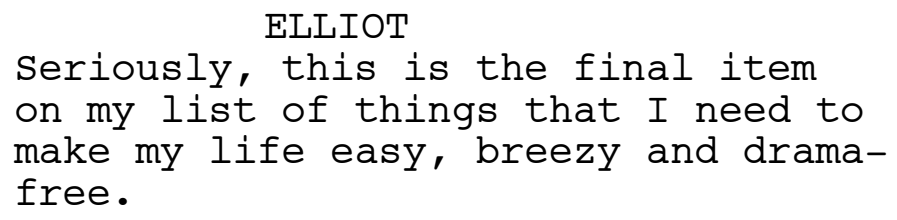

Elliot pulls out a scrappy bit of paper with faded handwriting on it. He hands it to Lester who reads the heading: "Elliot's Ten Year Plan."

ELLIOT

It's ten years this year, so

getting this job now would be perfect timing.

Lester frowns as he reads the list.

LESTER

Find a life-long partner to nest with.

Elliot leans in.

Check.

ELLIOT

Lester grimaces.

LESTER

Create the most comfortable bed

known to man.

Elliot wavers.

ELLIOT

Turns out that's more of an ongoing kind of thing. 
LESTER

Get a paying job that requires

little-to-no effort or

responsibility.

Elliot smiles and raises his eyebrows, suggestively.

LESTER

And that's it.

Lester, judgemental, grimaces at Elliot who nods proudly.

\section{LESTER}

Three lame things?

Elliot nods again, halfheartedly.

In ten years?

LESTER

Elliot tries not to get defensive.

Lester slides the bit of paper back over to Elliot.

LESTER

That's really sad, man.

Elliot frowns, and glances at the framed warrant of fitnesses hanging on Lester's wall but refrains from retorting.

Lester sighs, and pulls out a form from his desk.

Alrighty then.

LESTER

Elliot is hesitantly hopeful.

ELLIOT

What?

LESTER

It's all yours.

You serious?

ELLIOT

Lester doesn't know what to do with Elliot's enthusiasm.

LESTER

No one else has applied.

Elliot leaps off the swiss ball, which shoots off to the corner of the office.

Elliot goes after it, and clumsily rolls it back into place, but it gets punctured on a splinter on the floor, and slowly deflates before their eyes. 
Lester looks completely heartbroken.

Elliot flashes Lester an apologetic look

ELLIOT

Sorry. Should I...?

Elliot looks around not sure what to do about it.

Lester fights back tears.

LESTER

She was an old girl.

Elliot fidgets, uncomfortable and confused.

Lester can't control his tears any longer. He sobs uncontrollably.

ELLIOT

Christ. I'm so sorry....

Elliot, burdened by Lester's sadness, looks as if he's about to cry too.

Lester composes himself.

$$
\text { It's fine. It's fine. }
$$

Elliot looks relieved. He awkwardly changes the subject in an attempt to lighten the mood.

ELLIOT

Thank you so much for the job!

Elliot vigorously shakes Lester's limp hand.

EXT. CITY STREET - DAY

Elliot heads towards his scooter with a spring in his step.

As he mounts the scooter, he notices an ambulance parked up ahead where the collision still remains. A couple of

AMBULANCE OFFICERS haul a gurney, that carries a dead body covered in a sheet, into the back of the ambulance.

A crowd of people watch on in horror. Elliot, oblivious, pops his helmet on and hoons off down the street.

INT. FLAT, LOUNGE - DAY

A well-maintained, but severely lived-in, flat. The curtains are all closed. 
BARRY WALSH (65 - gruff kiwi bloke) is lying on the couch watching a reality dating show, while LEONE MILLER (48 motherly) is sitting at the table cutting out obituaries from a newspaper.

Leone reacts strongly to a particular obituary.

\section{LEONE}

Oh, Barry! Listen to this one.

Barry doesn't listen.

Leone brings the hole-filled paper closer to her eyes and reads off it.

LEONE

Pamela Hannett. 88. Beloved sister

to David, and doting Aunt to her

nieces. Lived life to make a

difference.

Leone shakes her head sadly.

\section{LEONE}

No husband or children. So sad.

Leone doesn't seem to mind that Barry is ignoring her. She cuts the obituary out, and puts it on a pile of other cut-out obituaries.

She continues reading when there is a knock at the door. Barry and Leone both sit up tensely, like there's been an earthquake or something.

The knock comes again - Barry and Leone glance at each other terrified.

\section{Jayesh?!}

JAYESH BANSAL ( 21 - hair slicked back with too much gel, and wearing a suit that's too big for him) appears from his bedroom.

Jayesh sees how tense Leone and Barry are.

$$
\begin{aligned}
& \text { What's up? } \\
& \text { LAYESH } \\
& \text { Lomeone knocked on the door. }
\end{aligned}
$$

Jayesh looks confused by this. He marches over to the door, and looks through the peep-hole.

Leone cowers over to the couch, but Barry doesn't give her room to sit so she awkwardly props herself on the arm of the couch. 
Jayesh opens the door, and drags in a box.

JAYESH

It's addressed to you, Leone.

Oh!

LEONE

Leone excitedly leaps off the arm of the couch and takes the box from Jayesh.

\section{Our Vibrofit!}

LEONE

Barry and Jayesh frown as they watch Leone rip into the box.

Vibro what?

BARRY

Leone pulls out an electronic board.

She puts it on the floor, and hits the power button. The board vibrates.

Leone stands on the board, and her whole body wobbles. She giggles at the sensation, and looks completely ridiculous.

Jayesh and Barry are watching her like she's lost her mind.

$$
\begin{aligned}
& \text { LEONE } \\
& \text { (vibrating voice) } \\
& \text { Want a go? }
\end{aligned}
$$

Jayesh and Barry both shake their heads. Leone looks disappointed, but stays on the vibrating board.

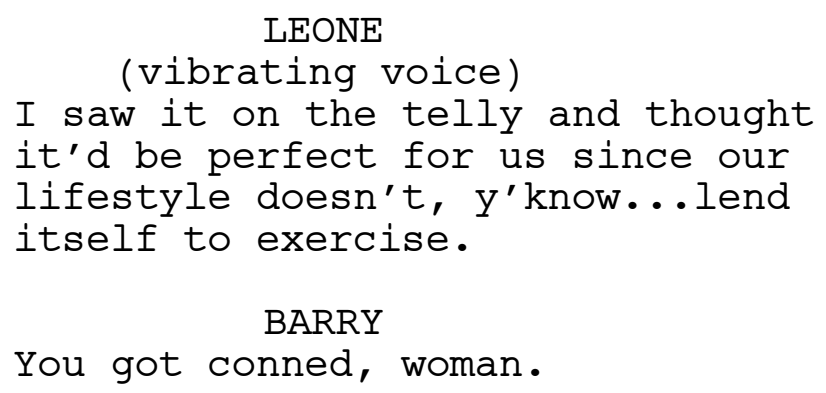

Barry resumes watching TV.

Leone looks offended. She glances at Jayesh for support. Jayesh shakes his head.

JAYESH

That ain't exercise.

Leone turns the board off, and steps off it - defiant. 
LEONE

Well, gee whizz. Here I was

thinking I was doing something nice

for the flat and this is the

thanks...

Leone trails off when she notices Jayesh is all dressed up.

\author{
LEONE \\ What's with the outfit?
}

Jayesh swiftly turns and scurries back into his room and slams the door behind him.

Leone follows after him, curious.

INT. FLAT, HALLWAY - DAY

Leone tries to open Jayesh's door but it's locked. She knocks.

$$
\begin{array}{r}
\text { LEONE } \\
\text { What's going on? }
\end{array}
$$

There is no response.

$$
\text { Jayesh?! LEONE }
$$

Leone hears Jayesh groaning, angrily.

$$
\begin{aligned}
& \text { JAYESH (O.S.) } \\
& \text { I'm going on a date tonight! }
\end{aligned}
$$

Leone looks absolutely mortified.

LEONE

A what?!

$$
\text { JAYESH (O.S.) }
$$

It's when two people do awkward

small talk so that they can live

the rest of their lives together in

comfortable silence.

Leone looks insulted.

LEONE

I know what a date is, Jayesh.

Coulda fooled me.

$$
\text { JAYESH (O.S.) }
$$

LEONE

We have a pact, in case you forgot.

Jayesh doesn't respond. 


\section{LEONE}

Are you planning on telling your socalled date the truth?

Still no response.

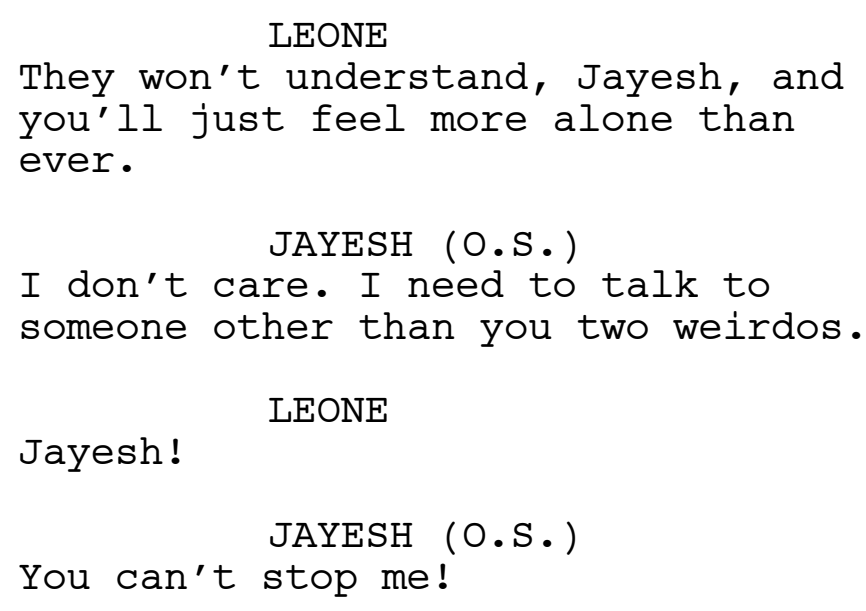

Leone thinks about this. She marches back into the lounge, and returns with a chair from the dining table.

Leone jams the chair underneath the door knob. She looks pleased with herself, and heads back into the lounge.

EXT. ELLIOT'S FLAT - DAY

Elliot parks his scooter in the driveway of a small, run-down flat.

He pulls out a bottle of champagne and two new wine glasses, still in their box, from the compartment beneath his seat. He skips inside.

INT. FLAT - DAY

Elliot enters a depressing bedsit with half its furnishings gone.

A Vibrofit is leaning against the wall next to the bed, which is stripped of its bedding.

Elliot frowns, confused.

\section{Russ?}

ELLIOT

The toilet in the adjoining bathroom flushes.

ELLIOT

Where's all our stuff gone? 
The bathroom tap can be heard being turned on

Elliot ignores the missing stuff for now.

ELLIOT

I got the job, Russ! Can you

believe it? I wish I could go smack

the smug smile off my primary

school principal who told my mum

and dad that I'd never achieve my

goals.

The tap is turned off.

\section{ELLIOT}

I bought us some wine to celebrate.

And wine glasses cos I feel like

our usual wine-in-a-cup wouldn't

quite...

The bathroom door opens, and TRINA WONG (17 - wearing a mishmash of bright colours) appears in the doorway.

Elliot gets the fright of his life, and drops the bottle of champagne on the floor. The glass shatters, and the wine fizzes out of the cracks.

ELLIOT

Who the heck are you?

Trina holds out her hand for Elliot to shake.

TRINA

I'm Trina.

Not wanting to make things awkward, Elliot shakes it.

Russ' cousin.

TRINA

Elliot frowns, still holding Trina's hand.

TRINA

He sent me here to break up with

you for him.

Elliot drops Trina's hand. All colour drains from his face.

What?

ELLIOT

TRINA

Russ doesn't want to be with you

anymore.

Elliot, confused, surveys all the missing furnishings. 


\section{ELLIOT}

But he's the only person I need.

Elliot's eyes well as the reality hits him. His eyes land on the bare bed, and he speaks in a trembling voice.

\section{ELLIOT}

He took my Bambillo pillow.

TRINA

Oof, I'm sorry. He said you'd enjoy

this whole situation cos you were

really supportive of his

Kickstarter for his break-up

business.

Elliot wipes his snotty nose with his sleeve, and slumps into the only chair left in the flat.

ELLIOT

I hated that stupid business idea

and that stupid Kickstarter

campaign.

Trina lets this sink in.

TRINA

Maybe that's why he's breaking up

with you, then.

ELLIOT

I never told him I hated it.

Trina ponders this too.

TRINA

Maybe that's why he's breaking up with you.

Elliot sinks further into his seat, more tears rolling down his face. He has a thought, and sits up again.

\section{ELLIOT}

Is this a test? For his break-up

business? He's just trying it out

to see if it will work?

Trina flashes Elliot a sympathetic look.

TRINA

This is the real deal, I'm afraid.

Elliot slumps back down in the chair, tears trickling down his cheeks.

Trina kneels down in front of him. 
TRINA

Don't waste those tears on him. My

parents are getting divorced and

it's the absolute worst. But it's

also the best. I mean, it was

horrible at the time, but now it's

OK. It just wasn't meant to be, you

know? And, trust me, time really

does heal all wounds. When one door

closes, another one opens.

Elliot's face is red, wet and snotty. His voice is choked up with tears.

\section{ELLIOT \\ Can you please leave?}

Trina looks severely disappointed.

TRINA

Was that no good?

Elliot gives her an exasperated look.

TRINA

Too many clichés?

Please leave.

ELLIOT

TRINA

It's just that I want to get good

at breaking up with people so that

Russ will hire me again. I read

online that when you're telling

someone something bad you should

show that you understand how

they're feeling and offer comfort.

I thought I did that, but I guess

it didn't work. Do you have any

tips for how I could improve?

Yes. Leave.

\section{ELLIOT}

Trina stands up again, but she doesn't make a move to leave.

TRINA

The thing is...Russ refused to pay

me for doing this, but he said that

you'd do me a favour in return.

Elliot scrunches up his face, disbelieving.

ELLIOT

I am not doing you a favour for

breaking up with me for him. 
Trina looks disappointed and panicked.

TRINA

But he promised.

Elliot flashes her a look of dismissal.

Trina kneels back down on the floor in front of Elliot, pleading.

TRINA

He said it was like a pay-it-

forward kind of thing. I do him a

favour, you do me a favour, someone

else does you a favour.

ELLIOT

The kid dies in that movie.

Trina crosses her arms and legs.

TRINA

I'm not leaving till you do me my

favour.

Elliot can't believe what he's hearing. He stands up and

tries to physically force Trina out the door, but he's too weak and Trina turns out to be surprising strong and quickly flips him over into a head-lock.

Elliot cries out as he struggles to break free.

Uncle! Uncle!

ELLIOT

Trina has no idea what Elliot is talking about. She grips him tighter.

Uncle!

ELLIOT

TRINA

What the heck are you talking

about?

ELLIOT

Y'know... Uncle.

Trina shakes her head at him.

TRINA

What does that mean?

Elliot thinks.

ELLIOT

I don't know. I heard it on a movie once. 
Trina baffled, doesn't loosen her grip. She eyes him threateningly.

ELLIOT

Fine! Stay here.

Trina smirks at her small victory, and lets Elliot go.

Exhausted, Elliot, picks himself up and flops down onto the beddingless bed.

\section{ELLIOT}

But I'm not doing you a favour.

INT. JAYESH'S BEDROOM - NIGHT

Jayesh's bedroom is messy despite his efforts to tidy it. The wall is covered in bits of blu-tac that had been holding up several posters of calendar girls that are now piled up on the floor.

The lights are out except for one that is specifically set up for a disco ball that emits dizzying patterns across the room.

Jayesh is anxiously sitting in front of his dressing table mirror, reciting lines from a stack of cue cards.

\section{JAYESH}

Is your name Summer? Cos you're

HOT!

Jayesh takes in a deep breath, puts the cue cards in his pocket, and goes to his door.

But when he tries to open it, he realises it's jammed and his face falls flat.

He slams his fist against the door.

$$
\text { JAYESH }
$$

Leone! !

INT. FLAT, LOUNGE - NIGHT

Leone body wobbles as she stands on the Vibrofit board at the dining table, reading obituaries again.

Let me out!

JAYESH (O.S.)

Barry, still watching TV, glances over at Leone.

BARRY

Let him go, Leone. 
Leone gives Barry a death stare.

LEONE

What about the pact?

BARRY

A man has needs.

Leone looks disgusted.

Barry finally sits up.

Leone steps over to him to stop him from getting up.

LEONE

This is going to have a negative

effect on all of us.

INT. JAYESH'S BEDROOM - NIGHT

Jayesh gives up on trying to get out of his door, so climbs out the window instead.

INT. ELLIOT'S FLAT - NIGHT

Elliot is still flopped on his bed, trying his best not to lash out at Trina who has turned the TV on and is flicking through the channels at an annoyingly rapid rate.

Trina looks at the time on her phone, and glances over at Elliot, concerned. Trina turns the TV's volume up.

Elliot snaps. He leaps up, rips the remote out of Trina's hand and turns the TV off.

ELLIOT

What's the favour?

Trina beams.

TRINA

I've been chatting to this guy online, right? And he wants to meet up with me tonight, but I don't think I want to go through with it. Like, I told him I wasn't sure I wanted to meet up just yet but he kind of kept pestering me - that's not a great sign is it? Part of me is curious cos he seems kinda cool, but most of me is just like

"blah!!" what if he's a psycho? Y'know?

Elliot blinks, mystified. 
TRINA

I also told him I'm slightly older and more experienced than I really am.

Elliot doesn't move.

\section{TRINA}

All I need is for you to meet up with the guy and tell him you catfished him.

Elliot laughs.

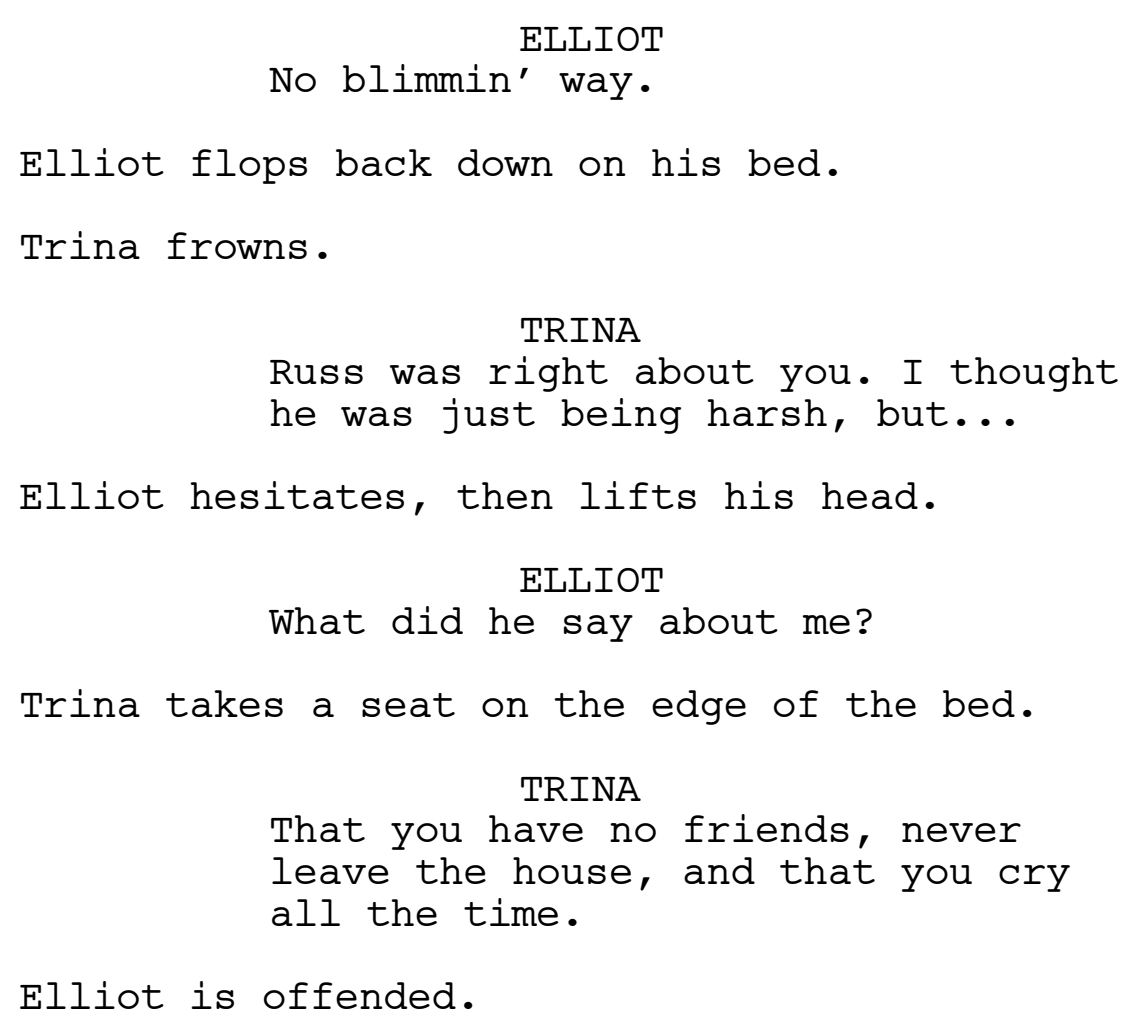

Elliot is mortified. He opens his mouth to defend himself, but he has nothing.

Trina hides her pleasure at having got Elliot right where she wants him. 
Trina stomps her foot on the ground, like she's just had an angry thought.

TRINA

He musta known you wouldn't do it!

He was just messing with me! That

shithead...

Trina checks to see that Elliot is taking her reversepsychology bait before taking the remote back off him.

She flops back down in the seat. She is about to flick the TV on again when Elliot has a thought, and takes the remote out of her grasp again.

\section{ELLIOT \\ Will you tell him I did it?}

Trina smiles to herself.

ELLIOT

Cos if I do it, and you tell him I

did it, then he'll see he's got me

all wrong, and he might take me

back.

Trina holds her hands together.

\section{TRINA}

That, my friend, is a very good plan.

EXT. RESTAURANT - NIGHT

A high-brow restaurant - everyone inside is dressed in suits and gowns. Live piano music can be heard drifting from within.

Trina and Elliot stop outside.

ELLIOT

You couldn't have chosen somewhere

a bit more...poor?

He chose it.

TRINA

Trina pulls out her phone and brings up the dating profile of Jayesh. He's dressed as The Joker, terrifying face paint and all, and he has self-titled himself as "Jayesh - the joker".

TRINA

This is who you're looking for.

Elliot tries to conceal his judgement. 


$$
\begin{gathered}
\text { ELLIOT } \\
\text { What if he's "The One"? }
\end{gathered}
$$

Trina shakes her head, not sensing Elliot's sarcasm.

TRINA

If it's meant to be, we'll meet

again in other circumstances.

Elliot looks skeptical, but this is soon over-run with

feelings of dread for what he's about to put himself through.

$$
\text { Good luck! TRINA }
$$

He wipes his clammy hands on his pants, then steps into the restaurant.

INT. RESTAURANT - NIGHT

A low murmur of polite chatter accompanies the live piano performance.

Elliot, completely out of place, scans the restaurant and spots a YOUNG INDIAN GUY sitting alone in the far corner of the restaurant, scrolling on his phone.

Elliot weaves through the tables - other diners glance at him with subtle judgement.

Elliot nervously takes a seat at the table, which is wobbly and not dressed like the other tables. The young Indian guy looks up at Elliot, confused.

Elliot speaks quickly and nervously.

$$
\begin{aligned}
& \text { ELLIOT } \\
& \text { I'm really sorry, but Trina isn't } \\
& \text { real, I pretended to be her } \\
& \text { because. . well... I really liked } \\
& \text { your profile picture and I thought } \\
& \text { maybe you'd-- } \\
& \text { YOUNG INDIAN GUY } \\
& \text {--What's going on? } \\
& \text { ELLIOT } \\
& \text { I'm the one you've been chatting } \\
& \text { to, not Trina. }
\end{aligned}
$$

The young Indian guy squints at Elliot, then stands up. Elliot sees he's wearing a uniform and apron.

$$
\text { I'm just a waiter. }
$$

$$
\text { YOUNG INDIAN GUY }
$$


Elliot is mortified, and only now realises that the table he's at isn't even part of the dining area.

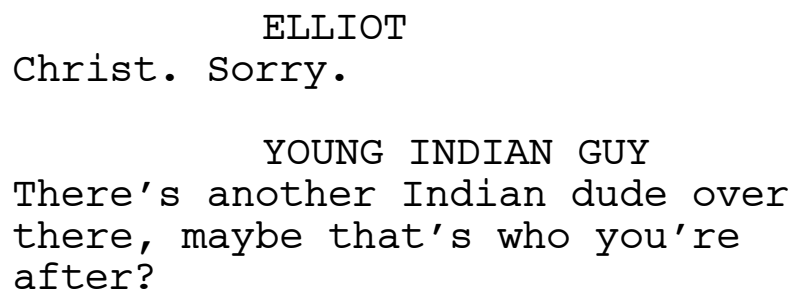

Elliot grimaces, embarrassed, as he looks across the restaurant and sees Jayesh sitting on the other side of the restaurant, looking very skittish.

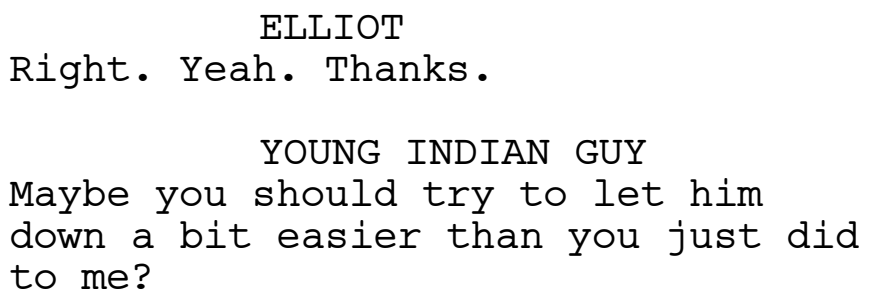

The waiter disappears into the kitchen, and Elliot makes his way over to Jayesh's table - even more rattled now.

When Elliot reaches the table, Jayesh looks up, wide-eyed and confused. Elliot takes a seat.

Jayesh looks around the room, suspiciously. He leans in and whispers to Elliot.

$$
\text { JAYESH }
$$

Dude, am I being Punk'd or

something? Cos I'll totally go

along with it, but I'd like to know

now so I can stick to a character.

ELLIOT

I don't think Punk'd is a thing

anymore.

Jayesh looks a bit disappointed.

JAYESH

Really? What about Pimp My Ride?

Elliot shakes his head, unsure.

$$
\text { MTV cribs? }
$$

JAYESH

Elliot shrugs again. Jayesh sinks in his seat.

JAYESH

My flatmate hogs the TV so I don't know what's on anymore. 
Elliot grabs a piece of bread from the middle of the table and bites into it. He chews and chews excessively.

\section{JAYESH \\ What are you doing?}

ELLIOT

I read somewhere that we're meant to chew our food 32 times to break everything down into smaller parts so the digestive system doesn't have to do as much work.

JAYESH

I meant what are you doing at my table, man?

Elliot is about to explain when their waiter - the young Indian guy from before - arrives, and hands them the menus. He smiles coyly at Elliot.

\section{WAITER \\ Can I get you drinks to start?}

Jayesh hesitates, Elliot uncharacteristically takes charge.

$$
\text { ELLIOT }
$$

A bottle of red please.

Jayesh's eyes go wide again.

The waiter nods and leaves them to it.

Jayesh leans in and whispers again.

\section{JAYESH}

You're paying for that, right?

Elliot hadn't thought this through.

Shit.

\section{ELLIOT}

JAYESH
Cos the only thing I can afford
here is the bread...
ELLIOT
Why'd you pick this place then?
JAYESH
I've been told their wagyu sandwich
is to die for and I thought my I
could impress...

Jayesh shakes his head - realising he doesn't have to explain himself. 
JAYESH
Where's my girl, Trina, man?!

Elliot clears his throat, fidgets a lot, then begins to speak when the waiter arrives with their wine. He pours it into the glasses, excruciatingly slowly.

WAITER

Would you like to hear the

specials?

Nah.

JAYESH

Yes!

ELLIOT

The waiter doesn't know what to do.

Jayesh can't contain his humiliation any longer.

JAYESH

What's going on, man?!

The waiter, sensing the tension, backs away and gives Elliot a knowing wink.

WAITER

I'll give you a minute.

Wait!

ELLIOT

The waiter stares at him.

ELLIOT

I've changed my mind - I don't want

the whole bottle.

Elliot tries to hand the bottle over, but the waiter doesn't accept it and instead uses the opportunity to give him a cryptic message.

WAITER

It's already been opened, sir, you

can't just abandon it now.

Elliot frowns angrily at the waiter.

ELLIOT

Just give me some time, OK?!

The waiter backs away again. Elliot is sweating profusely.

Elliot skulls his wine back in one go. He puts the glass down, fills the glass to the brim and skulls that back. He stares at a black smudge on an otherwise-white napkin, and takes a deep breath. 
ELLIOT

Trina doesn't exist, Jayesh. I was

pretending to be her because I was

so taken by your profile picture

and...

Elliot squirms when he sees how crushed Jayesh looks.

$$
\text { Oh, god... ELLIOT }
$$

Elliot trails off, shaking his head to himself. Jayesh is crushed.

JAYESH

I been catfished?!

The other diners in the restaurant, having overheard Jayesh's outburst, are staring at Elliot and Jayesh, and whispering amongst themselves.

\section{JAYESH}

What about all that stuff you told me about how when you were at high school those mean girls threw bloody tampons at you and all that? That was some elaborate shit, man.

Elliot doesn't know what to say.

A diner at the table next to them leans over and speaks directly to Elliot.

MALE DINER

People like you are the worst.

Elliot frowns, perplexed.

\section{Excuse me?}

ELLIOT

Elliot's perplexity intensifies when he sees that most people in the restaurant - including the waiters and chefs - are now giving him death stares.

\section{FEMALE DINER}

Son, you're worse than a window washer who washes your windscreen even though you've clearly mouthed "no cash!"

Other diners agree with this comment.

ELLIOT

Come on now, it's not that bad... 


\section{YOUNG DINER}

My Great Grandma got catfished. And

then she killed herself!

The waiter approaches the table, awkwardly.

$$
\begin{aligned}
& \text { WAITER } \\
& \text { I'm going to have to ask you to } \\
& \text { leave, sir. }
\end{aligned}
$$

Elliot is humiliated.

$$
\text { Seriously? }
$$

The waiter shrugs, apologetic.

$$
\begin{aligned}
& \text { WAITER } \\
& \text { Manager's rules. }
\end{aligned}
$$

Elliot looks to Jayesh who is overwhelmed by this whole thing but seems less upset than everyone else.

Elliot speaks to Jayesh, but he makes sure everyone else can hear.

$$
\begin{aligned}
& \text { ELLIOT } \\
& \text { Trina hired me to tell you that I } \\
& \text { was catfishing you cos she was too } \\
& \text { scared to meet you herself. }
\end{aligned}
$$

Jayesh let's Elliot's statement sink in, while everyone in the restaurant lets out a unanimous groan.

$$
\begin{aligned}
& \text { DINER } \\
& \text { Yeah, right! } \\
& \text { Fice try. }
\end{aligned}
$$

Everyone boos at Elliot, until Elliot can handle it no more.

\section{ELLIOT}

Oh, like you're all so perfect!

Elliot stands up and some diners throw food at him.

As Elliot wipes the food off, a DINER makes a chicken noise at him. This, for some reason, sparks other diners to make different animal noises - moos, baas, barks, and meows.

Elliot, disbelieving, scurries out of the restaurant. 
EXT. RESTAURANT - NIGHT

Trina sits on a bench, alone and bored. Every time someone walks past she thinks it might be Elliot but then she quickly shrinks back down when she realises it's not.

Finally Elliot comes out, looking completely frazzled.

She launches off the bench and goes to Elliot's side.

$$
\text { How'd it }
$$

TRINA

How'd it go?

\section{ELLIOT}

No great, Trina!

Elliot marches on.

Trina follows after him when she sees Jayesh exit the restaurant.

\section{Shit!}

TRINA

Trina panics, and ducks behind a LOSER CRUISER - a beat-up minivan.

Excuse me!

JAYESH

Elliot stops and turns to Jayesh who runs up to him.

JAYESH

Is what you said true?

What?

\section{ELLIOT}

JAYESH

That Trina's real?

Yeah...

ELLIOT

Trina, crouching in the gutter, can't believe what she's hearing.

\section{ELLIOT}

It's not that she doesn't like you.

She told me she does. It was just

too soon for her. So, maybe don't

pressure her so much, and she might

come around.

Elliot shoots Trina an intense stare.

Jayesh nods, relieved. 
JAYESH

You wanna come back and chill at my place?

Elliot is taken aback.

$$
\text { Oh, um... ELLIOT }
$$

Elliot hesitates, but catches a glimpse of Jayesh's pleading eyes and looks guilty.

\section{Sure. \\ ELLIOT}

Trina shakes her head, disbelieving.

Jayesh gestures to the loser cruiser.

This my ride.

JAYESH

Jayesh heads over to it.

Trina mouths "shit" to herself, then crawls behind the next parked car.

Jayesh and Elliot get in the car. Jayesh starts the engine, and his stereo blasts electronica music that fills the entire street. Elliot cowers in the passenger seat as they drive off.

Trina pulls herself up, and watches in disbelief as the car disappears in the distance.

INT. FLAT, LOUNGE - NIGHT

Leone and Barry are playing a competitive game of scrabble in the lounge.

Barry has his feet on the Vibrofit board, and his whole body pulses.

$$
\begin{gathered}
\text { LEONE } \\
\text { Chocka is not a word, Barry. } \\
\text { BARRY }
\end{gathered}
$$

It bloody is! Chock-a-block. Chocka-block full a fun.

Jayesh lets Elliot in through the front door. Leone and Barry look upon Elliot with shock horror.

LEONE

What's going on? 


\section{BARRY}

I thought you said he was in his room?

JAYESH

This is my buddy, Elliot. Elliot

these are my flatmates, Barry and

Leone.

\section{ELLIOT \\ Nice to meet you.}

Barry and Leone shoot Elliot chilly glares. Elliot stiffens.

$$
\text { We're gonna hang in my room. }
$$

Jayesh pulls Elliot down the hallway.

Elliot hears Barry and Leone whispering aggressively to each other.

INT. JAYESH'S BEDROOM - NIGHT

Jayesh flicks the light on, and the disco ball emits its dizzying patterns again.

\section{ELLIOT \\ What's up with your flatmates?}

Elliot sits on Jayesh's bed, which has rose petals scattered over it. He looks a bit spooked when Jayesh locks his bedroom door.

\section{JAYESH}

Don't worry about them. You wanna hear me tell some jokes?

Jayesh takes a seat at his desk and opens his laptop.

$$
\text { Uh, OK... }
$$

\section{ELLIOT}

Jayesh scrolls through a website on his laptop, and laughs sporadically.

He clicks a play button which sets off a continuous loop of the Seinfeld theme that's interspersed with canned laughter.

Jayesh tries but usually fails to time his jokes so that they fit into the rhythm of the theme song and laughter.

Can a kangaroo jump higher than a house? 
Jayesh looks to Elliot with a huge grin on his face. Elliot shrugs to half-heartedly go along with it.

Jayesh looks back at his laptop to read the punch line.

\section{JAYESH}

Of course, a house doesn't jump at

all.

Jayesh slaps his knee with glee. The laugh track plays a bit too late.

$$
\begin{aligned}
& \text { JAYESH } \\
& \text { Man! I got you! }
\end{aligned}
$$

Elliot gives him a sympathy laugh.

\section{JAYESH}

I really wanna be a comedian, $\mathrm{y}^{\prime}$ know?

\section{ELLIOT \\ Yeah? You ever done any shows?}

More canned laughter.

Jayesh ignores Elliot's question. He reads off the computer again, but tries hard to look like he's memorised it.

\section{JAYESH}

A pessimist says to an optimist:

"Things just can't get any worse!"

And the optimist says: "Of course

they can!"

Elliot smirks ever-so-slightly. Jayesh is thrilled.

See!

$$
\text { JAYESH }
$$

ELLIOT

$$
\text { Very funny. }
$$

More canned laughter.

Jayesh goes back to his computer and scrolls through the jokes again.

\section{ELLIOT}

So what do you do? For, like, a job and that?

Jayesh's eyes go wide at another joke that he reads off his screen.

JAYESH

Why did the can crusher quit his job? 


\section{Why?}

The canned laughter drowns out Jayesh's punchline.

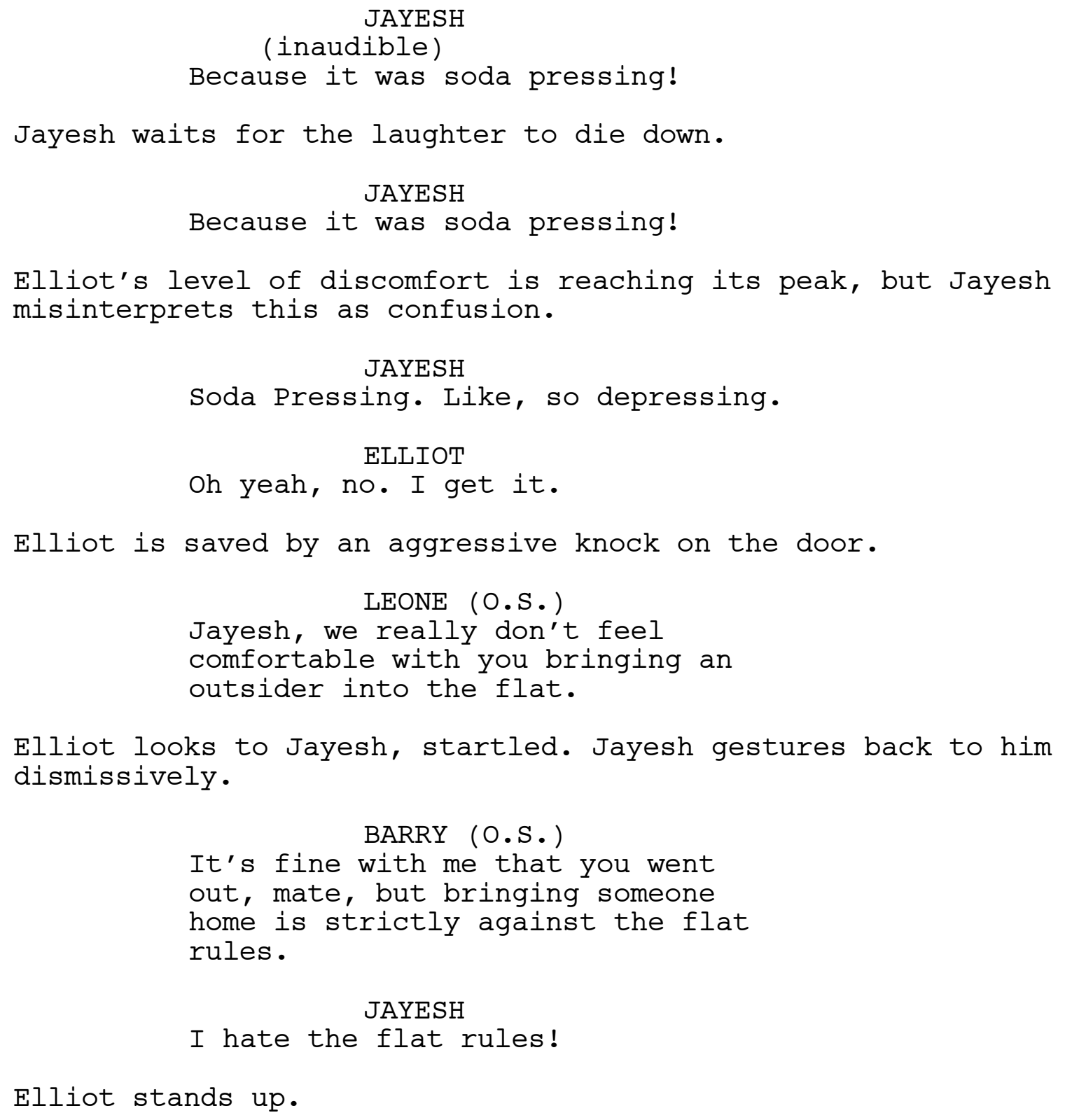

Elliot stands up.

$$
\text { It's fine. I'll go. }
$$

Elliot makes a move for the door, but Jayesh stops him.

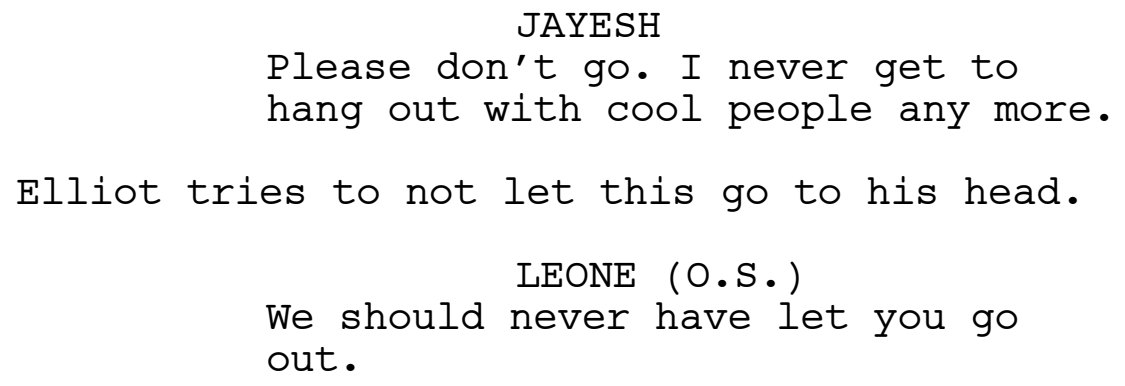


ELLIOT

Is this a cult or something?

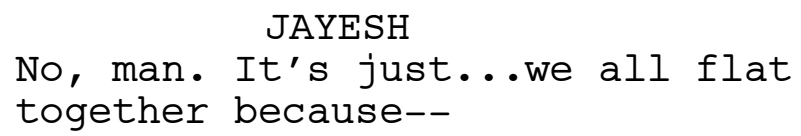

--Barry and Leone proceed to slam their fists against the door to drown out Jayesh's words.

$$
\begin{gathered}
\text { LEONE (O.S.) } \\
\text { Don't you dare tell him! } \\
\text { BARRY (O.S.) } \\
\text { He won't get it! }
\end{gathered}
$$

Defiant, Jayesh yells over their knocking.

\section{JAYESH}

We're all murderers!

The knocking stops.

$$
\text { Jayesh! LEONE (O.S.) }
$$

Elliot is completely freaked now.

ELLIOT

What do you mean?

BARRY (O.S.)

We're not actual murderers!

LEONE (O.S.)

Why would you say that, Jayesh?

The door knob rattles.

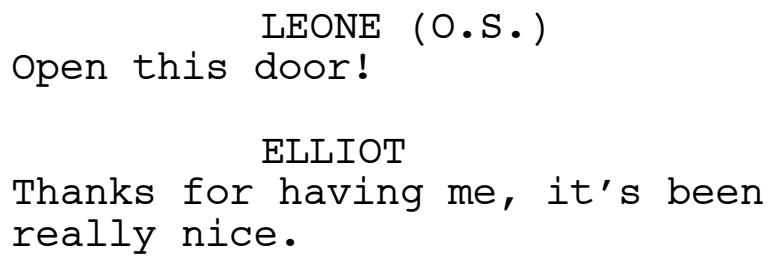

Elliot lunges for the door, and unlocks it.

The door swings open, and Elliot scurries past Barry and Leone.

Elliot bolts for the front door.

LEONE

Wait! Please!

Elliot turns to Leone and Barry who hover behind him. Jayesh keeps his distance. 
Barry shoots daggers at Jayesh.

BARRY

We're not bloody murderers!

Barry shifts his gaze to Elliot.

\section{BARRY}

Murderers kill on purpose.

Elliot stiffens again. His voice is high pitched.

ELLIOT

What the heck does that mean?

LEONE

We live together because each of us accidentally killed someone.

What?

\section{ELLIOT}

LEONE

Barry, here, was painting a little

old lady's house but accidentally

used leaded paint and the fumes

killed her.

Barry looks solemnly down at his feet.

BARRY

Never got paint on the cheap again.

Elliot has gone slightly limp at hearing this.

LEONE

A few years ago Jayesh cooked his family a nice meal for his beloved grandfather's birthday. But everyone got food poisoning, and

his grandfather was too frail to recover from it.

Jayesh's eyes brim with tears.

\section{JAYESH}

Thought if I salted the chicken

enough, it wouldn't matter that it was expired.

Elliot is overwhelmed.

\section{And you?}

\section{ELLIOT}

Leone fidgets uncomfortably. Elliot sees a framed photograph of Leone with her SISTER going skydiving. He shudders. 
BARRY

She doesn't like to talk about it to outsiders.

LEONE

People just don't understand the pain we're in.

ELLIOT

What about your families?

All three of them fall silent for a moment.

BARRY

My wife'd always say, "It's not

your fault, Bazza. You've got to get over it."

Jayesh steps forward.

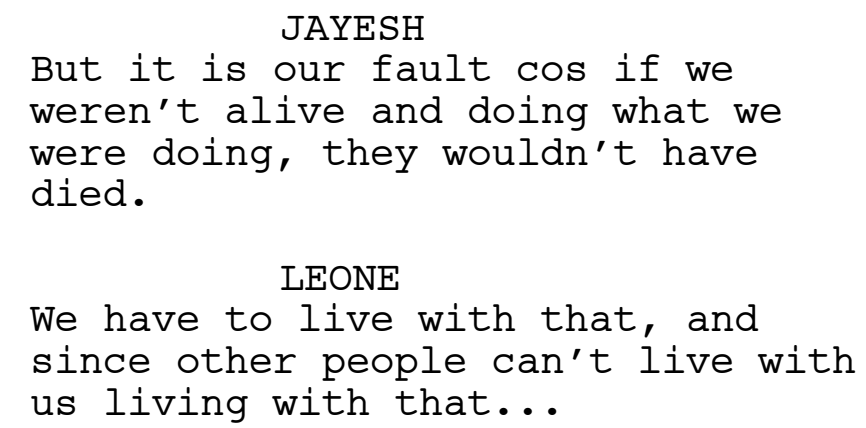

Elliot has seen the Vibrofit board, which continues to hum.

ELLIOT

You cut yourselves off from the

rest of the world?

LEONE

See, it's impossible for anyone

else to understand.

\section{ELLIOT}

No. I get cutting yourself off. I really do.

Leone, Barry, and Jayesh all look like a tiny weight has been lifted.

EXT. ELLIOT'S FLAT - NIGHT

Jayesh's loser cruiser pulls up in Elliot's driveway. Elliot hops out, and heads into his flat.

Jayesh drives off in the distance. 
INT. ELLIOT'S FLAT - NIGHT

Trina is sitting on the lone seat watching TV when Elliot enters and jumps in fright again.

$$
\text { Oh, sweet Jesus. }
$$

Trina flicks the TV off.

$$
\text { TRINA }
$$

What the hell was that?

\section{ELLIOT}

I'm not telling you anything until

you give me Russ' key back.

Elliot holds out his hand. Trina reluctantly hands the key over.

\section{So?}

TRINA

Elliot considers the question as he takes a seat on his bed.

\section{ELLIOT}

He was nice. Not a psycho. I mean,

he has a bit of baggage and a

serious joke problem - two things

that may or may not be connected -

but he seems like a good person.

Trina smiles to herself.

$$
\text { TRINA }
$$

Is he still interested in me?

ELLIOT

Ask him yourself.

Elliot gets up, and opens the door.

ELLIOT

Can you please leave me alone, now?

Trina sighs, then pulls herself up. As she heads out the door, she turns to Elliot.

TRINA

$$
\begin{aligned}
& \text { Thanks for doing it. } \\
& \text { ELLIOT } \\
& \text { I'm weirdly glad I did. }
\end{aligned}
$$

Elliot smiles at Trina, grateful. But then his smile turns into a wince. 
ELLIOT

Please don't tell Russ how much I

cried.

TRINA

I wouldn't dare give him the

pleasure.

Elliot looks relived.

A hint of guilt spreads across Trina's face.

TRINA

Oh, but...just so you know... he did

actually pay me.

What?

ELLIOT

TRINA

See ya!

Trina darts outside. Elliot watches her disappear, stumped.

INT. ELLIOT'S FLAT - LATER

Elliot is lying on his bed, staring at his laptop. He has a window up on the screen, displaying Russ' Kickstarter campaign for his break-up business. The business has only received $8 \%$ of its $\$ 3000$ funding goal.

Elliot clicks on the video and watches Russ' low-budget ad:

RUSS (30s - very well-groomed) is sitting in the foetal position in the middle of a room fake-crying.

Russ stops fake-crying, then looks straight into camera.

RUSS

Don't you hate it when you make someone cry like that?

Russ stands up.

$$
\begin{aligned}
& \text { RUSS } \\
& \text { Well, if you donate to this } \\
& \text { campaign, you won't have to. }
\end{aligned}
$$

Russ gestures to a link at the bottom of the video.

RUSS

If I get enough money, I can make

your loved ones cry for you!

Elliot stops the video, pulls out his credit card from his wallet, and pays for the entire remaining amount. 
He writes a message before submitting the payment: "I'm sorry I wasn't supportive of you xxx."

He hits the "pay now" button, then snaps the laptop shut.

He lies back on his bed, satisfied. He pulls an unzipped sleeping bag over his body, and goes to sleep.

INT. ELLIOT'S FLAT - MORNING

Elliot wakes up to his alarm, shivering. He checks his phone and is disappointed to see he has no messages.

He checks Russ' Kickstarter campaign, which now says "Goal Reached". But there is no response to his comment.

Annoyed, Elliot gets dressed while intermittently checking his phone.

INT. BASEMENT - DAY

Lester leads Elliot into a windowless basement full of boxes of files and guides him towards a scanner in the corner.

Elliot surveys the basement with a dread-filled gaze.

$$
\text { LESTER }
$$

Be prepared to start talking to

this thing like it's a person.

Lester pats the scanner. Elliot looks weirded out.

\section{LESTER}

It's what happened to the last one who quit.

Lester pulls a small jar of vitamins out of his pocket, and hands it to Elliot.

\section{LESTER}

And I suggest you take these for your vitamin D.

Elliot looks down at the pottle, anguished.

Lester slaps Elliot on the back.

\section{Good luck!}

$$
\text { LESTER }
$$

Lester leaves Elliot alone with his regret.

He checks his phone, but sees that there's no signal down here. 
He holds his phone up in the air and walks around like a goon until he finds a corner where he gets a bar. He places his phone precariously on top of a pile of boxes.

Elliot sighs, and gets to work.

EXT. OFFICE BUILDING/ STREET - EVENING

Elliot's scooter is parked in a legal park this time.

Elliot, exhausted, appears from the building and is blinded by the natural light.

He checks his phone once more, and becomes furious when there's still nothing from Russ.

He stands next to his scooter, and starts to draft a text that says "Did you get....", when:

Excuse me!

MOTHER

Elliot looks up and sees the mother of the toddlers from the start approaching him.

MOTHER

Is that your scooter?

Elliot nods, flummoxed.

The crazed mother looks ready to bite his head off.

MOTHER

You killed someone!

What?

\section{ELLIOT}

MOTHER

Yesterday you parked in my carpark so I had to park in a loading zone

and a truck had to park in a

driveway and then a guy had a

flippin' heart attack!

Elliot is flabbergasted.

MOTHER

You need to take responsibility for

your actions!

Elliot fumbles onto his scooter, and hoons away from the mother - rattled. 
INT. ELLIOT'S FLAT - NIGHT

Elliot, curled up on his bed, continues to check his phone until he's had enough.

He sits up and calls Russ's number.

He waits, anxiously for Russ to answer.

Hello?

RUSS

Elliot frowns.

ELLIOT

Russ. It's me.

RUSS

Oh.

ELLIOT

You deleted my number?

So?

RUSS

Elliot doesn't know how to respond to this.

ELLIOT

Have you checked your Kickstarter

page?

Yup.

RUSS

ELLIOT

Oh.

RUSS

What?

ELLIOT

Nothing, it's just...

RUSS

You want a thank-you?

Elliot doesn't say anything.

RUSS

You do. You want a thank-you

because you're desperate.

Elliot's face crumples. 
RUSS

You're, like, the most useless

excuse for a person that I ever met

and I don't know why I wasted so

much time being with you.

Elliot lets out a whimper.

ELLIOT

That's not nice.

Russ laughs.

RUSS

You just gave me all your money and

now I'm bashing you and that's all

you have to say?

Elliot is too taken aback to be angry.

ELLIOT

Can I please have my money back?

Russ laughs again.

RUSS

Nope.

The phone goes dead. Elliot throws the phone one bed.

Fuck!

ELLIOT

Elliot curls into a ball on his bed, and sobs - until he sees the Vibrofit leaning against the wall.

He sits up, wipes his tears, determined.

INT. FLAT, LOUNGE - NIGHT

Barry is sitting in his underwear watching a soap opera on TV while dropping in and out of sleep.

There is a knock on the door. Barry sits up with a jolt.

The knock comes again. Barry stands up, nervous, and creeps over to the door.

Leone and Jayesh appear from their bedrooms, looking like they've just been woken up.

Barry hesitantly looks through the peep hole.

BARRY

It's that weedy boy from the other

night. 
Jayesh perks up.

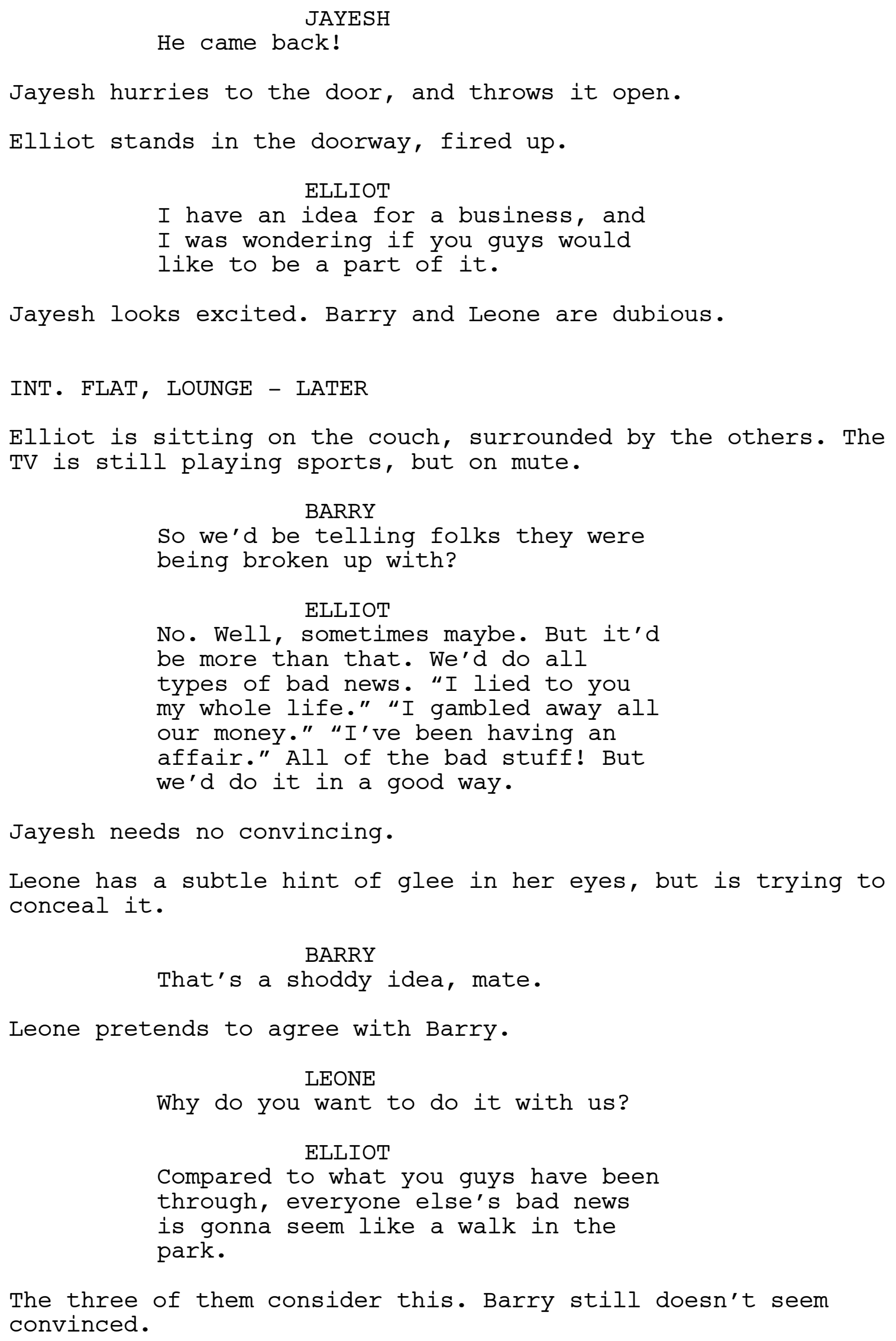

\section{BARRY}

That's a shoddy idea, mate.

Leone pretends to agree with Barry.

LEONE

Why do you want to do it with us?

ELLIOT

Compared to what you guys have been

through, everyone else's bad news

is gonna seem like a walk in the

park.

The three of them consider this. Barry still doesn't seem convinced. 
JAYESH

I can ease people's pain with my humour.

Elliot winces a little.

\title{
Yeah.
}

ELLIOT

Leone can't help herself.

LEONE

I am very in tune with people's emotions.

Barry scoffs. Leone snaps back, offended.

$$
\text { LEONE }
$$

Barry'll be no good cos he's too much of a brute.

\section{Bollocks!}

BARRY

Leone raises her eyebrows, taunting.

LEONE

And he hasn't left the house in five years.

Barry fidgets.

\author{
BARRY \\ What happened to keeping to \\ ourselves?
}

Leone hesitates. Elliot steps in.

\section{ELLIOT}

I thought it might be a good opportunity to assimilate back into

the world. For me too, I mean. My whole life I've avoided shit I thought would make me miserable, but it's really just led me to other shit that's made me miserable.

Jayesh's mind is blown.

\section{JAYESH}

We can stop avoiding shit, by helping other people avoid their shit!

Elliot doesn't look so sure about this interpretation, but can't deny it. 
Exactly.
LELIOT
What a relief it would be to focus
on other people's mistakes and pain
for once.
Barry scoffs again.

Exactly.
LELIOT
What a relief it would be to focus
on other people's mistakes and pain
for once.
Barry scoffs again.

BARRY

Exactly - you only wanna do it cos

you love sticking your snout in

other people's drama.

Leone crosses her arms, offended.

LEONE

That's not true!

ELLIOT

So is that a yes?

Yeah, man! JAYESH

Leone is about to say yes when Barry says:

BARRY

Nah. Not for me, mate.

Barry un-mutes the TV, and slinks back down into his seat.

Leone does a silent thumbs up.

INT. FLAT, JAYESH'S BEDROOM - LATER

Jayesh and Leone are crowded around Elliot who is working on Jayesh's laptop, writing up an ad on TradeMe:

BUSINESS NAME: Bad News Itd.

ABOUT YOUR SERVICE: GOT BAD NEWS? DON'T WANT TO BREAK IT? DON'T WORRY, WE CAN DO IT FOR YOU! CALL US ON 02109458745 NOW!

Elliot hovers the cursor over the "submit ad".

JAYESH

(singing badly)

Hit the button and let me know!

Elliot and Leone grimace at Jayesh. Elliot hits the button to shut him up. The three of them smile, cheer, and give each other gumby high-fives. 
INT. FLAT, LOUNGE - NIGHT

Barry hears the cheers, and glances down the hall with a hint of jealousy.

He gets up and waddles to Jayesh's bedroom.

INT. JAYESH'S BEDROOM, FLAT - NIGHT

Elliot, Leone and Jayesh turn to the door when they hear Barry knocking.

LEONE

Yes?

BARRY (O.S.)

You'll make a bloody mess of it without me.

The three of them smile, and hear Barry's trudging footsteps heading back into the lounge.

INT. BASEMENT - DAY

Elliot's phone is back on the pile of boxes again. Elliot keeps stopping his work to check the TradeMe ad on his phone for new views; there are three in total.

INT. FLAT, LOUNGE - DAY

Jayesh and Leone are also refreshing the ad, on Jayesh's laptop. The only major difference between them and Elliot is they are both wearing t-shirts with "Bad News Ltd." scrawled over their fronts in vivid.

Barry has had it with the incessant mouse click.

\section{BARRY}

Would you stop that? You're going

to break the damn thing.

Leone keeps refreshing the page, but with a softer touch.

\section{JAYESH}

I can't believe we haven't had any calls yet.

$$
\text { Can't you? }
$$

INT. ELLIOT'S FLAT - NIGHT

Elliot, disheartened, refreshes the TradeMe ad on his laptop, which is now at nine views. 
There is a knock on his door.

Elliot, confused, goes to the door. He looks through the peep hole, but no-one is there.

Elliot opens the door, and squeals in terror when he sees a creepy papier-mâché version of his head sitting on the step. A knife is lodged in its temple, and tomato sauce oozes out of it. A sign around its neck reads:

\section{"Take down the ad or you're dead - Russ"}

Elliot gathers himself, astounded. He scans the area for Russ. He picks up the head, and carries it outside.

EXT. FLAT - NIGHT

Elliot goes to dispose of the head in the rubbish bin at the side of the house, but is distracted by the detail and craftsmanship.

He is inspecting the head closely when he gets another fright of his life -- Trina is standing there, looking guilty.

\section{ELLIOT}

Do you not have a home or something?

\section{TRINA}

I'm really sorry about the head. I wasn't actually gonna put it there but Russ made me get proof.

Trina pulls out her phone and shows Elliot the picture of him looking freaked out at the head.

\section{ELLIOT}

I didn't realise he had such a knack for papier-mâché.

\section{TRINA}

He really hates you.

ELLIOT

Yeah, well, turns out that's nothing new.

TRINA

No, he's properly angry. Like, he's seriously about to take you down.

Elliot looks genuinely freaked now, but tries to hide it.

\section{ELLIOT}

He can't scare me into submission

any more. 
Trina looks concerned that Elliot isn't getting it.

TRINA

He once had this friend, Jean, who accidentally bought the same

cardigan as him and now Jean is in

a mental hospital.

Elliot processes this.

ELLIOT

That's what he means when he says

he's going to "Jean" someone?

Trina nods.

ELLIOT

I always thought it meant he was

going to fit someone with denim.

Trina shakes her head at Elliot.

ELLIOT

How did I not see how horrible he is?

Trina shrugs, like she's a little bit proud of Russ.

TRINA

We come from a long line of

manipulators.

Trina snaps back into serious mode.

TRINA

Anyway, he's going to "Jean" you if

you don't take the ad down.

Trina sees in Elliot's stunned expression that he's finally getting it.

TRINA

I'll see ya round.

Trina starts walking away, but she stops and turns to look at him.

TRINA

Hopefully •

Elliot, dazed, watches Trina disappear down the street.

INT. ELLIOT'S FLAT - NIGHT

Elliot is lying on his bed, wide awake, staring at his laptop which displays the Bad News ad. 
He hovers the cursor over the "remove listing" button. He finally clicks it and a pop up tells him the listing has been permanently removed.

INT. APARTMENT - NIGHT

Jayesh and Leone are in the exact same position as before, still refreshing the page, when the ad disappears and they get a "page cannot be found" message.

Jayesh and Leone gasp, and glance at each other, horrified. Jayesh tries to refresh it, but the same message comes up.

JAYESH

I'll call Elliot.

INT. ELLIOT'S FLAT - NIGHT

Elliot's phone rings. He answers it, apprehensively.

$\begin{array}{lc}\text { Hello? } & \text { ELLIOT } \\ \text { Is this Bad News? } & \text { CALLER (PHONE) } \\ & \text { ELLIOT } \\ \text { Uh... } & \end{array}$

INT. LIVING ROOM - NIGHT

MANDY (30s), the passerby from the start, is sitting at a wobbly table. She speaks into her phone.

On the table, Tyche - the dog, also from the start - lies dead in a box.

\section{MANDY}

Cos I was kinda out taking a dog for a walk the other day, but the dog got spooked by a car accident and she escaped her leash and I lost her for ages and I just found out she sorta got hit by a car and I really really really don't want to have to tell the owner cos he's this sweet old man with a heart of gold.

INT. ELLIOT'S FLAT - NIGHT

Elliot is listening with a look of trepidation on his face. 


\author{
MANDY (PHONE) \\ I mean, if you could pretend to be \\ the one that hit him or something. \\ Make out like it was all your \\ fault. Shit, that would be so \\ amazing! \\ Elliot remains silent. \\ MANDY (PHONE) \\ Do you do that kind of thing? \\ Elliot swallows his nerves. \\ ELLIOT \\ You bet.
}

END OF EPISODE. 



\section{BAD NEWS}

Episode Two:

" HEALBOI "

Written by

Alison McLachlan 

EXT. RESIDENTIAL STREET/ STATE HOUSE - DAY

MAGNUS (13 - wearing his scruffy school uniform) power-walks down the footpath with his backpack slung over one shoulder.

He walks up a path to a run-down house.

Magnus flings his backpack onto the front porch of the house. He sits--determined--on the cracked concrete steps, and gazes down the end of the street waiting for someone, or something.

Eventually his MOTHER (worn out, and still wearing pyjamas) comes out of the house, and leans in the doorway. She lights a cigarette.

MOTHER

Watcha doing, Maggie?

Magnus doesn't take his eyes of the street.

$$
\begin{aligned}
& \text { MOTHER } \\
& \text { How was school? } \\
& \text { Fine. }
\end{aligned}
$$

The mother takes a drag on her cigarette - frowning.

MOTHER

You got any homework?

No.

MAGNUS

The mother tries to follow her son's gaze.

MOTHER

What are you looking at?

Leave me alone.

MOTHER

Geez Louise, someone's been eating

the mean beans...

Magnus shuffles down a step - but still keeps his eye on the street.

A baby cries inside the house. The mother puts her cigarette out, and goes back inside.

Magnus thinks he sees the thing he's waiting for, and stands up. But soon realises it's just a cat.

He sits back down, dejected. 
Several hours go by - the sun goes down, and the street lamps light up. Magnus doesn't move from his step except to scrunch into a smaller huddle to keep himself warm.

Magnus' little SISTER pokes her head out the door.

$$
\text { Tea time, Magnus. }
$$

Magnus doesn't move.

$$
\text { MAGNUS }
$$

Not hungry.

The sister hesitates.

$$
\text { SISTER }
$$

Can I eat your fish fingers?

\section{Whatever.}

\section{MAGNUS}

The sister can't believe her luck - she runs back inside, excited.

More time passes - it's even darker now, but Magnus still hasn't moved.

His mother calls out to him from inside.

$$
\begin{gathered}
\text { MOTHER } \\
\text { Magnus - get your arse in bed! }
\end{gathered}
$$

Magnus hears his Mother's footsteps approaching, he pulls himself up then dashes down the street.

As he runs he approaches a douchey-looking sports car with the numberplate, "HEALBOI".

Magnus stops at the car, and rips the side mirrors off angrily.

\section{TITLES.}

EXT. STREET/ PRIMARY SCHOOL - DAY

Trina (wearing the uniform that Magnus was wearing) is walking her two younger sisters - LILY (7) and HARRIET (10) to the primary school gate where other kids are being dropped off by their parents.

TRINA

Make sure you come straight to the

gate after school this time. We

don't want a repeat of last week. 
Trina sees Lily and Harriet roll their eyes at each other.

HARIIET

You mean when you made Lily's

teacher cry cos we were three

minutes late and you accused her of

murdering us?

TRINA

Am I not allowed to worry?

Harriet and Lily scrunch their faces up.

LILY

You're not our mum!

Harriet and Lily scurry off into the school, without a goodbye.

\section{LOVe you too! \\ TRINA}

Harriet and Lily ignore her. Trina makes eye contact with a MOTHER.

$$
\text { Kids, am I right? }
$$

The mother looks at her like she's a weirdo, but soon comes around.

\section{MOTHER}

My little Sophie peed on my face

this morning. On purpose.

Trina grimaces, and goes back to watching Harriet and Lily walking away, and sees Harriet and Lily showing LILY'S FRIEND (7) a stuffed toy pony that Lily has taken out of her backpack.

Trina's whole demeanour changes. Her eyes go wide, and her cheeks redden. She marches over to Lily.

TRINA

What the hell are you doing with Victor?!

Lily hugs the worn out and deformed, but realistic, soft-toy pony close to her and it lets out a creepy, mechanical whinny.

$$
\text { I'm taking him for show and tell. }
$$

Trina shakes her head furiously. She tries to pry Victor out of Lily's arms, but Lily dodges her. 
TRINA

Victor isn't just some thing you

can take for show and tell you

monster!

Trina notices how everyone, including passersby, are looking at her with trepidation.

Trina sees a weed in the garden.

TRINA

Take this instead.

Trina leans over and tries to yank the weed out of the ground, but it doesn't budge and it has nasty prickles on it.

She picks a smaller and even more pathetic off-cut.

Trina tries to shove the limp weed into Lily's hand, but she doesn't take it and it flops onto the concrete.

LILY

It's animal show an tell.

TRINA

Yeah well, animals sometimes eat weeds.

Lily isn't convinced.

(to Lily)

HARRIET

You know she sucks on its ear when

she's stressed out, right?

Lily sees the wet, worn out ear. She grimaces and looks at Trina who nods in shameless affirmation.

Lily hands Victor over.

Trina, relieved, holds Victor close to her, like it's her baby.

TRINA

Good day to you!

Trina turns on her heels and marches off, and ignores the judging glare that the mother shoots her.

Lily, Harriet and Lily's friend are left bewildered.

LILY'S FRIEND

What was that about?

HARRIET

Our mum gave it to her when she was

little. 
Lily's friend nods, acknowledging.

LILY'S FRIEND

She's really not handling the

divorce well is she?

Harriet and Lily shake their heads. The three of them head off into school, but Lily stops in her tracks.

Lily scampers back to where they were standing, picks up the weed from the ground, and rejoins the other two.

INT/EXT. LOSER CRUISER/STREET - DAY

Jayesh is slowly driving Leone and Elliot down a residential street. They pass the sports car that now has its sidemirrors ripped off, as well as several dents.

Elliot is staring out the window, looking for an address.

Unbeknownst to any of them a unmarked police car is on their trail.

INT. UNMARKED POLICE CAR - DAY

A FEMALE POLICE OFFICER (40s - earnest) is driving, and a MALE POLICE OFFICER (50s - anxiously fidgeting all the time) is in the passenger seat.

FEMALE POLICE OFFICER

If you were a sex trafficker, wouldn't you get a less creepy

vehicle to do your business in?

The male police officer doesn't respond.

FEMALE POLICE OFFICER

I would get a less creepy vehicle.

INT/EXT. LOSER CRUISER/STREET - DAY

Elliot points to a house, which MANDY is standing in front of.

There it is!

ELLIOT

Jayesh pulls over.

The unmarked police car parks up at a distance. 
EXT. OLD MAN SAM'S HOUSE - DAY

Elliot, Leone, and Jayesh watch as Mandy bends over and picks up a medium-sized package, wrapped in a Council rubbish bag, and tied up with a dog collar.

A dog's tail pokes out of a hole.

\section{This is Tyche.}

The three of them eye the bag, grossed out.

MANDY

Sam, the owner, told me to leave

him to roam in the backyard til he

gets back this afternoon.

Mandy holds the bag out for Elliot. Elliot reluctantly takes it.

MANDY

I've dug a wee hole under the fence

to make it look like she escaped on

her own. All you need to do is come

back in an hour or so with Tyche,

and act like you ran her over.

Mandy pulls out an a wad of twenty-dollar notes.

MANDY

Here's your hundred.

Mandy sighs.

\section{MANDY \\ That's how much I got for looking after her.}

Leone snatches the money before she's even handed it over.

Mandy gently places her hand on the rubbish bag.

\section{Sozzy •}

MANDY

She heads off down the driveway. Jayesh has a thought.

$$
\begin{aligned}
& \text { JAYESH } \\
& \text { Leave us good feedback, please! }
\end{aligned}
$$

It's unclear as to whether Mandy has heard.

Leone and Elliot are grimacing.

What?

$$
\text { JAYESH }
$$




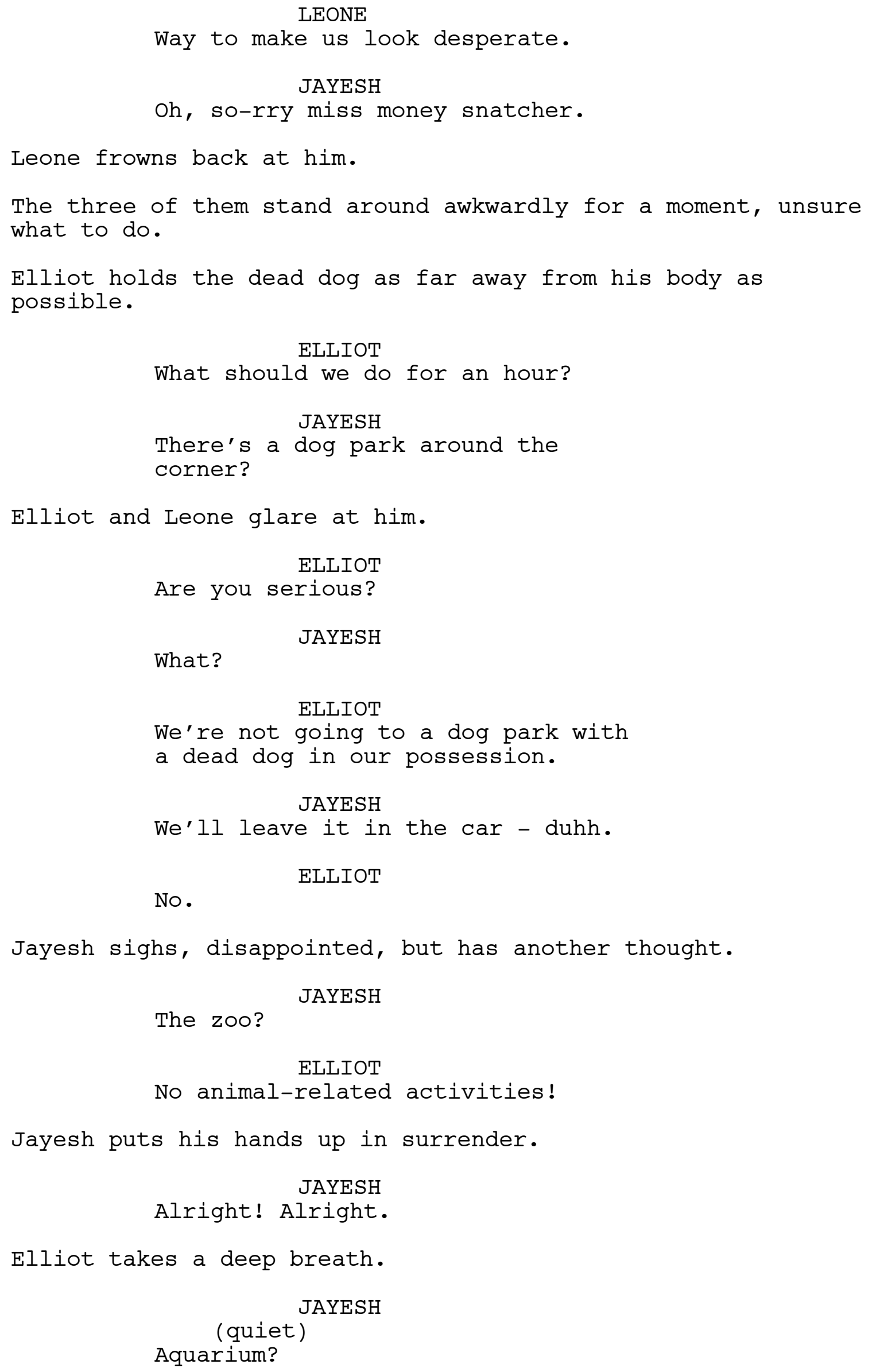


Elliot looks like he's going to flip out, so Leone steps in. LEONE

Wait in the car?

They head to the loser cruiser, which is parked in front of the unmarked police car.

EXT. HIGH SCHOOL QUAD - DAY

Trina is eating lunch alone, surrounded by groups of friends chatting and laughing with each other.

Trina is on her phone, searching for the Bad News ad. She frowns when she sees several pornographic images have been posted on the ad, along with headings like, "All yours for low price".

Trina continues scrolling down the page and realises it's been changed into a sex trafficking business just as a GROUP OF BOYS walk past her and one BOY accidently kicks her bag, sending Victor skidding across the ground.

When he stops skidding he makes the robotic whinny.

Mortified, Trina lunges for Victor but one of the boys picks him up before she can.

$$
\text { What the hell is this? }
$$

Trina tries to snatch Victor off him, but he passes it to his friend.

$$
\begin{aligned}
& \text { TRINA } \\
& \text { Please give him back. } \\
& \text { Him? }
\end{aligned}
$$

The boys continue to pass Victor around as it continues to whinny.

Other STUDENTS gather around to watch.

Trina, humiliated, sees a TEACHER passing by •

\section{Miss!}

TRINA

The boys all roll their eyes at each other as they watch Trina run up to the teacher, nearly in tears.

TRINA

$$
\text { Miss - they stole my toy pony. }
$$


The boy who now holds Victor throws him up into a nearby tree.

The teacher frowns and looks over at the boys, then back at Trina.

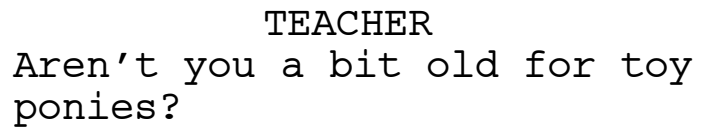

Trina, mortified, watches as the teacher continues on her way.

The students all snigger at Trina as she marches back over to the boys.

$$
\text { Where is he? }
$$

The boys all shrug and head off. Trina looks around for Victor, until a FEMALE STUDENT points up into the tree where Victor hangs precariously.

$$
\text { Victor! I'm coming! }
$$

Trina scrambles up the tree like an idiot, not caring about the scene she's making, and also not noticing that she tears the side seam of her school shirt on a branch.

She grabs onto Victor, but she missteps and falls off the branch she's on.

\section{TRINA \\ Agghhhhhh!}

Trina closes her eyes and grips onto another branch with her one free arm, until her feet are dangling about an inch above the ground - but she doesn't realise it.

$$
\text { Agghhhhhh! TRINA }
$$

The surrounding students all glance at each other, disbelievingly.

A FEMALE STUDENT steps up to her.

\section{FEMALE STUDENT}

Let go, I'll catch you.

Trina takes in a deep breath and lets go as the female student walks away.

Trina grimaces and groans as she hits the ground much faster than anticipated.

The students all laugh at her. 
Trina picks up her bag, carefully places Victor inside it, and hurries out of the school - mortified but trying hard to act like nothing happened.

INT. LOSER CRUISER - DAY

Elliot is sitting in the front passenger seat, anxiously shifting his gaze between the clock on the dashboard and old Man Sam's house.

Jayesh is playing pea-knuckle with himself, and is losing somehow.

Leone is sitting in the back, with the door open, nodding off to sleep.

Elliot spots a taxi pull up outside Old Man Sam's house. Elliot sits up straight.

There he is!

ELLIOT

The others look over at the house, and see OLD MAN SAM (80, frail and friendly-looking) being assisted into the house by the TAXI DRIVER.

Leone clutches her heart in exaggerated pain.

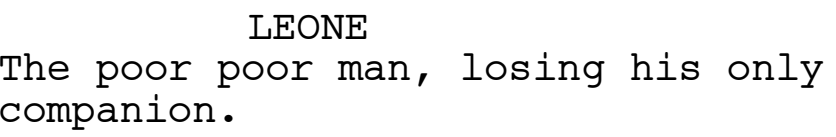

Elliot's eyes are tearing up at the sight of Old Man Sam.

\section{ELLIOT}

I don't think I can do this.

Jayesh whacks him on the shoulder with the back of his hand.

JAYESH
Course you can - you did it to me.
ELLIOT
Yeah, but - we're dealing with real
human emotion here.

Jayesh looks offended.

JAYESH

I'm real human emotion.

$$
\begin{gathered}
\text { Yeah, but-- } \\
\text { ELLIOT } \\
\text { LEONE } \\
\text {--Good lord! }
\end{gathered}
$$


Jayesh and Elliot turn to Leone, who has a panicked look on her face.

What?

JAYESH

LEONE

Tyche's gone.

What?!

ELLIOT

Elliot cranes his neck to see.

How? !

ELLIOT

Leone looks guilty. She points out the open door, into the gutter.

LEONE

I put her out there cos she was

stinking up the car.

Elliot leaps out of the car, and surveys the perimeter. There's no sign of the dead dog.

\author{
LEONE \\ What kind of weirdo steals a dead \\ dog?
}

Elliot stands in the spot where Tyche should be.

ELLIOT

What are we gonna do?

The three of them all look panicked, as the taxi drives off in the background.

INT/EXT. OLD MAN SAM'S HOUSE - DAY

Old Man Sam waddles into his house. Mandy has left a note on the table that says:

"Tyche's out back - Mandy".

Old Man Sam heads to a sliding door at the back of the house.

He opens it up, and scans the well-maintained back garden.

old Man Sam makes a little whistle sound.

$$
\begin{aligned}
& \text { OLD MAN SAM } \\
& \text { Tyche! Here girl. }
\end{aligned}
$$

Old Man Sam bends over to slap his knee. 


$$
\begin{gathered}
\text { OLD MAN SAM } \\
\text { Where's my beautiful girl? }
\end{gathered}
$$

Old Man Sam makes his way outside, quickly becoming disheartened.

$$
\text { Tyche? }
$$

$$
\text { OLD MAN SAM }
$$

Old Man Sam lets out a more urgent whistle, but stops when he sees Mandy's hole under the fence line.

Old Man Sam hobbles over to it, using all his energy and muscle to lower himself down.

He looks through the hole and gets his hopes up when he sees the paws of a dog, but when he looks closer he realises it's just the neighbour's dog, panting and staring at him.

Old Man Sam's eyes brim with tears.

EXT. STREET - DAY

Jayesh, Leone and Elliot are at opposite ends of the street, searching for the dead dog in people's wheelie bins.

The unmarked police car is parked up in the distance.

Elliot's phone rings and he answers, panic-stricken.

$$
\text { Hello? }
$$

ELLIOT

EXT. BUS STOP - DAY

Trina, dishevelled, sits in a bus stop talking on her phone. Victor sits on her lap.

\section{TRINA}

Why didn't you take the ad down?

Trina?

$$
\text { ELLIOT (PHONE) }
$$

$$
\text { TRINA }
$$

I warned you.

$$
\text { ELLIOT (PHONE) }
$$

What are you talking about?

$$
\text { TRINA }
$$

You haven't seen it?

$$
\text { ELLIOT (PHONE) }
$$

Look, I'm actually really busy

right now, so... 
Trina sits up, curious.

TRINA

Busy doing what?

A case.

ELLIOT ( PHONE)

Trina can't believe it.

TRINA

You're still doing the business?

You've got more guts than I

thought.

ELLIOT (PHONE)
Yeah, well....we're kind of in a jam

so if you don't mind...

Trina has a thought that fills her with excitement and hope.

TRINA

I'm not doing anything right now...

OK..?

ELLIOT (PHONE)

TRINA

I can help. I'm great in a crisis.

ELLIOT (PHONE)

That's really not a good idea.

TRINA

Nonsense. Where are you?

ELLIOT (PHONE)

Truly, we don't need you.

Trina tries not to be hurt by this.

TRINA

Please?

No!

ELLIOT (PHONE)

Trina hears an ice cream truck jingle over the phone.

$$
\begin{aligned}
& \text { ELLIOT (PHONE) } \\
& \text { I've got to go. Bye. }
\end{aligned}
$$

Elliot hangs up - and Trina looks completely deflated. She brings Victor to her nose and sniffs him then, after checking that no one is around, she sucks on his ear for comfort.

She stops sucking on the ear when she hears an ice cream truck in the distance and then sees it driving past. 
She leaps up, puts Victor back in her bag, and heads in the direction that the ice cream truck came from.

EXT. STREET - DAY

Elliot puts his phone away, filled with dread, and searches through the wheelie bin in front of him.

Jayesh now has eight ice creams in his hand. He tries to hand one over to Elliot.

Elliot frowns at him.

$$
\begin{aligned}
& \text { ELLIOT } \\
& \text { Um, no thanks. }
\end{aligned}
$$

Jayesh deflates.

JAYESH

Leone? Sweet treat?

Leone also frowns at him, and continues searching through the bins.

Jayesh takes a seat on the kerb, and consecutively shovels all eight ice creams into his mouth then gets back to searching the bins, fighting through the brain freeze.

INT. UNMARKED POLICE CAR - DAY

The two police officers in the car are curiously watching Jayesh, Elliot, and Leone.

\section{FEMALE POLICE OFFICER \\ What are they doing?}

The male police officer tilts his head when he sees Jayesh pull out a gorilla slipper from a bin and hide it behind his back with glee.

$$
\begin{aligned}
& \text { MALE POLICE OFFICER } \\
& \text { Get to know the kids by their } \\
& \text { rubbish so it's easier to groom } \\
& \text { them? }
\end{aligned}
$$

The female officer isn't quite convinced. They both continue watching.

EXT. STREET - DAY

Jayesh yells to the others, the slipper still behind his back. 
Elliot and Leone look worried about the answer.

$$
\text { What..? LEONE }
$$

Jayesh holds up a dilapidated gorilla slipper.

$$
\text { JAYESH }
$$

A slipper!

$$
\text { Oh, thank god. }
$$

Jayesh grins from ear to ear. Elliot and Leone nod, acknowledging the joke but not laughing at it.

$$
\begin{aligned}
& \text { JAYESH } \\
& \text { You'ld've laughed if I had my } \\
& \text { background music. }
\end{aligned}
$$

Elliot has a change of tune.

$$
\begin{gathered}
\text { ELLIOT } \\
\text { Wait. That's perfect! }
\end{gathered}
$$

Elliot runs over to Jayesh, and rips the slipper off him, shoves it into a plastic bag, and proceeds to jam rubbish into the slipper to give it some weight.

Elliot holds the bag out to show the others.

ELLIOT

We can make a fake dead dog.

Jayesh clearly thinks this is a brilliant idea, but Leone is more tentative.

$$
\begin{aligned}
& \text { LEONE } \\
& \text { Or maybe we should just tell Mandy } \\
& \text { we lost the damn dead dog? }
\end{aligned}
$$

Jayesh and Elliot are both horrified by this suggestion.

$$
\begin{aligned}
& \text { JAYESH } \\
& \text { And risk getting negative feedback } \\
& \text { on TradeMe? } \\
& \text { ELLIOT } \\
& \text { It'd be the end of us, before we } \\
& \text { even began. }
\end{aligned}
$$

Leone puts up her hands in surrender.

OK, OK. We'll make a fake dead dog.

Leone shakes her head. 
LEONE

But we're gonna need something more

convincing than that.

Leone points to the pathetic package in Elliot's hands, just as a limp banana peel flops out of it.

INT. OLD MAN SAM'S HOUSE - DAY

Old Man Sam is sitting at his table, eyes red from crying. He dials a number on his landline, and waits for a response.

Old Man Sam's voice is quivery.

$$
\text { OLD MAN SAM }
$$

Mandy. Tyche's escaped, and I don't

know what to do!

$$
\text { MANDY (PHONE) }
$$

(restraining her outrage)

What's that now?

EXT. UNMARKED POLICE CAR - DAY

Trina rounds the corner - past the smashed-up sports car from the beginning - and is thrilled to see Elliot in the distance.

Trina's about to run up to him, but stops - right outside the unmarked police car - when she notices her ripped shirt.

INT. UNMARKED POLICE CAR - DAY

The police officers watch on in horror as Trina ties the two flaps of fabric in a knot on the side of her waist so that she looks like she could be in a Britney spears music video.

$$
\text { Oh, god. }
$$

MALE POLICE OFFICER

The male police officer looks nervous.

MALE POLICE OFFICER

What should we do?

FEMALE POLICE OFFICER

I mean, it's still not proof.

The male police officer nods, relieved that they don't have to act on it yet. 
EXT. STREET - DAY

Trina starts marching confidently down the street towards Elliot who is horrified to see her. He hurries over to her before the others see her up close.

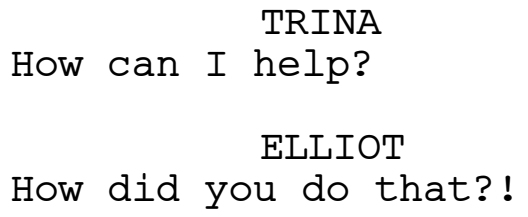

Trina finally sees the reason for Elliot's resistance Jayesh, sitting on the footpath, sifting through a pile of rubbish, oblivious to Trina.

Trina is outraged.

$$
\text { What - are you BFFs now? }
$$

Elliot looks guilty.

ELLIOT

I told you - not a good idea.

Trina thinks about this, but shakes her head.

TRINA

No. You know what? I'm sick of pretending all the time.

Elliot's cringes as Trina walks over to Jayesh.

Trina glances shyly at Jayesh who looks up at her but doesn't seem to recognise her.

Jayesh looks a bit creeped out by her staring.

$$
\mathrm{Hi} \text {. }
$$

TRINA

Jayesh looks confused.

\section{Hi?}

JAYESH

Trina - and Elliot - can't believe it. Trina's confidence vanishes in an instant.

Elliot joins them.

$$
\begin{gathered}
\text { ELLIOT } \\
\text { Jayesh - this is-- } \\
\text { TRINA } \\
\text { (mortified) } \\
\text {--Beyoncé. }
\end{gathered}
$$


Elliot raises his eyebrows.

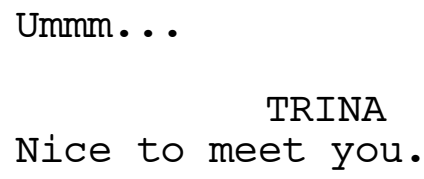

Jayesh smiles.

$$
\begin{gathered}
\text { JAYESH } \\
\text { Your name is Beyoncé? }
\end{gathered}
$$

Trina nods, anxiety-ridden.

$$
\text { Man, your parents must be cool. }
$$

Leone comes over.

$$
\begin{aligned}
& \text { ELLIOT } \\
& \text { Bold, more like. That's like } \\
& \text { calling your kid God. } \\
& \text { TRINA } \\
& \text { They named me when she was still } \\
& \text { with Destiny's Child, before she } \\
& \text { really hit it big with her solo } \\
& \text { work. }
\end{aligned}
$$

Elliot eyes Trina sternly. Trina doesn't budge.

$$
\text { Hello, there? }
$$

$$
\text { LEONE }
$$

Trina puts out her hand for Leone to shake, and cringes as she says:

$$
\begin{aligned}
& \text { Beyoncé. TRINA } \\
& \text { LEONE }
\end{aligned}
$$

Excuse me?

$$
\begin{gathered}
\text { JAYESH } \\
\text { Her name's Beyoncé! }
\end{gathered}
$$

Leone shakes Trina's hand, weirded out by her demeanour.

LEONE

Leone.

Trina notices the rubbish-filled slipper.

TRINA

Why are you shoving rubbish into a slipper? 
The three of them glance at each other, none of them wanting to admit to their situation.

$$
\begin{aligned}
& \text { ELLIOT } \\
& \text { We were meant to deliver a dead dog } \\
& \text { to its owner but someone left it } \\
& \text { out on the kerb and lost it. }
\end{aligned}
$$

Leone throws her hands in the air, offended.

$$
\text { LEONE }
$$

The smell of dead animal is

extremely hard to get rid of!

Trina frowns at the rubbish-filled slipper.

TRINA

You're trying to make a fake dead $\operatorname{dog}$ ?

The three of them all fall silent - ashamed of their silly plan.

$$
\text { JAYESH }
$$

You don't happen to have a dead dog do you, Beyoncé?

Trina laughs and shakes her head.

\section{ELLIOT}

Something that resembles a fake dead dog?

Trina's face falls and she tenses.

$$
\text { You mean, like a toy? }
$$

The three of them look hopeful.

\section{ELLIOT}

Yeah! A toy dog could work. Do you have one?

Trina hesitates, then laughs awkwardly again.

\section{TRINA}

No. Why would I..? That would be really weird if I...Toy dog.

Trina laughs.

The three of them deflate again. Trina takes a deep breath.

$$
\text { TRINA }
$$

Why don't you just buy one from a toy store? 
The three of them let this idea sink in, and then they all smile in unison.

INT. UNMARKED POLICE CAR - DAY

The police officers watch the four of them hurry down the street, leaving the discarded rubbish.

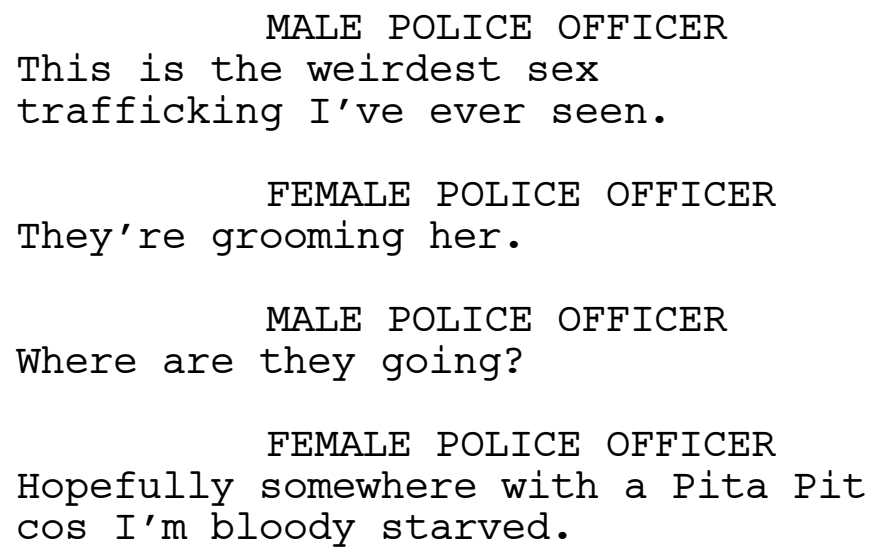

The female officer starts the engine.

EXT. TOY STORE - DAY

The unmarked police car pulls up in the toy store carpark, next to Jayesh's loser cruiser.

INT. UNMARKED POLICE CAR - DAY

The officers gawk at the toy store sign.

MALE POLICE OFFICER

Well this certainly doesn't look good.

The female officer looks around and sighs, disappointed. No Pita Pit.

FEMALE POLICE OFFICER

MALE POLICE OFFICER

Do you wanna go in, or...?

The male officer clearly doesn't want to go in. I'll do it. FEMALE POLICE OFFICER

The male officer looks relieved.

The female police officer steps out of the car, and heads into the store. 
INT. TOY STORE - DAY

Leone, Jayesh, Elliot and Trina are all scouring an aisle of soft toys, but none of them are much better than Jayesh's gorilla slipper.

The female police officer appears at the end of the aisle, and watches them inconspicuously.

Elliot sidles up to Trina who's taking her job of finding a toy dog very seriously.

\section{ELLIOT}

What's up with him not recognising you?

Trina grimaces. She whispers back.

TRINA

I don't know. And, quite frankly, I'm insulted.

Elliot has a thought.

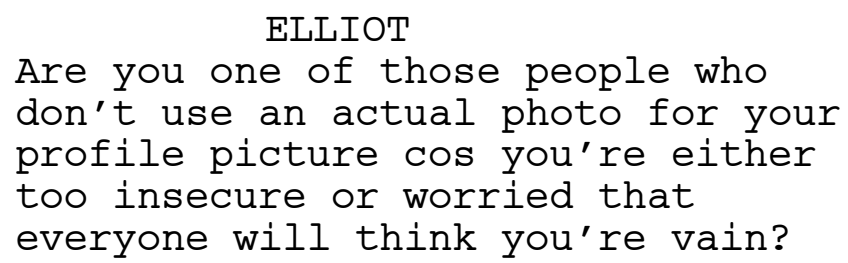

Trina frowns and shakes her head.

TRINA

No. Those people are the worst.

ELLIOT

Good. Yeah. I reckon.

Trina pulls out her phone, and shows Elliot her dating profile picture - over-filtered and photo-shopped, it looks nothing like her.

Elliot snorts with laughter.

What?

TRINA

ELLIOT

You look like a middle-aged woman.

Trina takes a closer look at her photo - self-awareness finally kicking in.

TRINA

What's wrong with me?

Elliot shrugs, still smiling to himself. 
Guys !

Jayesh reaches behind some teddy bears, to grab something he's seen.

The other three join Jayesh who, to Trina's horror, is holding out an exact replica of Victor.

Jayesh inspects it closely.

\section{JAYESH}

Perfect weight. All we'd have to do is wrap it up in a bag, smear it

with blood and tell him not to look inside. What do you reckon?

It's perfect!

\section{ELLIOT}

Leone notices the price tag.

LEONE

Um...how rich are you fullahs?

Leone points at the price tag which has $\$ 399$ on it.

\section{JAYESH \\ Goodness gracious!}

Jayesh puts the pony back on the shelf, like it's a bomb that's about to go off.

\section{ELLIOT}

Who the hell would pay that much

for something that creepy?

Trina laughs awkwardly.

\section{LEONE}

Look. I know we've all been

thinking it so I'm just gonna put

it out there... Let's just find a dog and kill it.

Everyone's eyes go wide and they fidget uncomfortably unsure whether Leone is kidding or not.

$$
\begin{aligned}
& \text { LEONE } \\
& \text { Jayesh - you said you know where a } \\
& \text { dog park is? }
\end{aligned}
$$

It seems that she's not kidding.

The female police officer is looking very confused. Trina is extremely uncomfortable now. 
JAYESH

What about if we got to the SPCA

and ask if they have any spare dead dogs?

Jayesh, Elliot, and Leone all glance at each other considering Jayesh's plan.

The female officer exits the store.

Trina looks baffled.

TRINA

Um. Maybe you could just tell the

owner that his dog is dead, but you

buried it already?

INT. UNMARKED POLICE CAR - DAY

The female police officer gets in the car, and both officers watch as the four of them get into the loser cruiser.

So?

MALE POLICE OFFICER

FEMALE POLICE OFFICER

Something about a stealing a dead dog?

The male officer frowns.

Is that code?

MALE POLICE OFFICER

The female officer shrugs.

The loser cruiser pulls out of the car park, and the police officers follow after them.

EXT. OLD MAN SAM'S HOUSE/ STREET - EVENING

The loser cruiser is pulled up on the road, outside old Man Sam's house. The unmarked police car is parked up behind it.

Elliot, Leone, Jayesh and Trina are standing at Old Man Sam's door.

ELLIOT

(a bundle of nerves)

OK. Let's do this!

Despite his words, Elliot doesn't move.

Jayesh eyes him, then steps up and knocks on the door himself. 
Old Man Sam eventually answers, and looks upon them with his blood-shot and tear-filled eyes.

The sight of Old Man Sam and is sadness causes Leone, Elliot and Jayesh to freeze up.

OLD MAN SAM

Can I help you?

Trina takes the lead.

TRINA

Are you missing a dog?

Old Man Sam is filled with hope.

OLD MAN SAM

Do you know where she is?

TRINA

I'm afraid we have some bad news.

Old Man Sam's hope fades.

What happened?

OLD MAN SAM

Um.

TRINA

Trina looks to the others - realising she doesn't know the story.

TRINA

What happened again, guys?

Elliot and Jayesh are still frozen. Leone steps up and starts jabbering.

\section{LEONE}

The poor little darling darted out

onto the road. We didn't have

enough time to brake, and she got

caught under our wheel. All we

heard was her yelp and a crunch as

her bones were obliterated and her

guts--

Trina whacks Leone on the arm.

LEONE

We're very sorry, but your dog is

dead.

Old Man Sam is completely defeated. He looks past the three of them, and eyes the loser cruiser. 


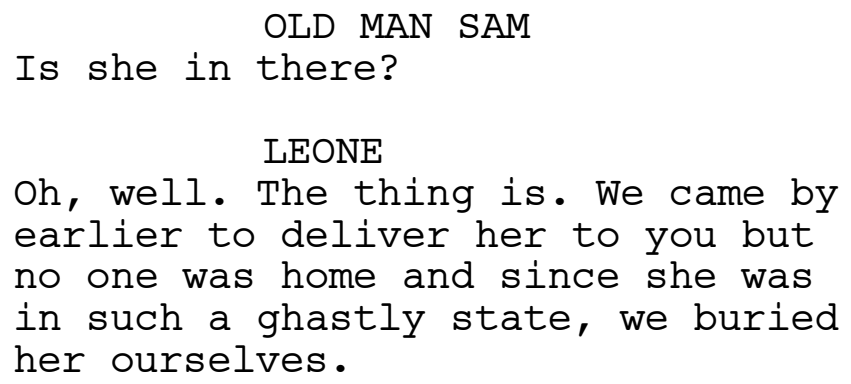

Old Man Sam looks furious.

You did what?

OLD MAN SAM

This is not the reaction they were hoping for.

TRINA

We gave her a nice send off.

OLD MAN SAM

Who the fuck do you think you are?!

Old Man Sam reached for an umbrella from behind his door, and takes a step towards them, threateningly. The four of them step backwards.

TRINA

Kidding! We're just kidding!

Old Man Sam looks even more furious. And the others are dumbstruck.

What?

OLD MAN SAM

LEONE

Yes. Isn't that a funny joke?

Buried your dog

ourselves... Whaaat??

OLD MAN SAM

So she's alive?

LEONE

No, no. Definitely dead. Very very

dead. Just not buried. We'll just

go get her.

Leone turns on her heels and scurries down the driveway, Elliot, Jayesh, Trina follow after her.

EXT. LOSER CRUISER - DAY

The four of them power walk to the car. 


$$
\begin{gathered}
\text { LEONE } \\
\text { Great plan, Beyoncé! } \\
\text { TRINA } \\
\text { At least I said something! }
\end{gathered}
$$

Trina glares at Jayesh and Elliot.

JAYESH

He reminded me of my dead Grandpa.

ELLIOT

That was really unpleasant.

They all tumble into the loser cruiser.

INT. LOSER CRUISER - DAY

The four of them sit in silence.

ELLIOT

What do we do now?

LEONE

You know what we need to do.

ELLIOT

We are not killing a dog, Leone!

Trina sees a bit of Victor poking out of her bag, and panics. She glances at her wrist - pretending there's a watch on it.

$$
\begin{gathered}
\text { TRINA } \\
\text { (fake urgency) }
\end{gathered}
$$

Shoot. Is that the time? I have to get going.

Elliot sees that old Man Sam is still watching them from his front step.

$$
\begin{aligned}
& \text { ELLIOT } \\
& \text { Drive, Jayesh. }
\end{aligned}
$$

Jayesh starts the engine, and Trina sees the actual time on the dashboard: $3: 03 \mathrm{pm}$.

TRINA

$$
\text { (genuine urgency) }
$$

Shit! Is that the time?! I have to get going!

Jayesh pulls away, and starts driving.

Trina stands up and before Jayesh has had time to stop properly, Trina launches herself out the door. 
JAYESH

What the hell?

INT. UNMARKED POLICE CAR - DAY

The two officers watch as Trina launch out of the loser cruise, and sprint down the street.

\section{FEMALE POLICE OFFICER \\ What did they do to her?}

The female police officer drives after Trina, while the loser cruiser drives off in the other direction.

EXT. TRINA'S HOUSE - LATER

Trina sprints down the street, with the same level of panic. She turns into her house.

INT. TRINA'S HOUSE - DAY

Trina bursts into the house.

TRINA

Harriet? Lily??

Trina heads into the lounge where Harriet and Lily are sitting on the couch - eyes glued to the TV.

$$
\text { Oh, thank goodness you're OK! }
$$

Trina kisses them on the tops of their heads.

TRINA

I'm so so so sorry I wasn't there

to meet you. How long did you wait?

Harriet turns her head.

Whaddaya mean?

\section{HARRIET}

TRINA

When I didn't turn up at the school gate?

Oh.

\section{HARRIET}

Harriet turns back to the TV.

HARRIET

We didn't wait. 
Trina is insulted.

TRINA

You didn't wonder where I was?

Harriet turns back to the TV.

Nup.

HARRIET

Lily isn't even listening - she laughs at something on the TV.

Trina looks dejected.

TRINA

Have you eaten? There's some fruit

in the fridge...

HARRIET

I made us an omelette.

Lily finally pipes up.

LILY

It was om-azing.

Harriet smiles at Lily's lame pun, and nudges her affectionately.

Trina is disturbed at how OK her sisters are without her.

She trudges out of the house, unnoticed.

INT. SPCA - DAY

Distance barks and meows can be heard from the dogs and cats in an adjoining corridor.

Jayesh leads a bumbling Elliot and Leone up to the reception desk of the SPCA.

$$
\begin{aligned}
& \text { ELLIOT } \\
& \text { I really don't think this is a good } \\
& \text { idea. }
\end{aligned}
$$

The desk is vacant so Jayesh rings the bell.

$$
\text { JAYESH }
$$

What else are they gonna do with them?

The RECEPTIONIST enters and smiles at them.

$$
\text { How can I help? }
$$

RECEPTIONIST 
Jayesh acts very casual and happy-go-lucky.

\title{
JAYESH
}

Yeah. Look. We were just wondering

if you have any dead dogs up for

grabs?

Elliot shakes his head at Jayesh's wording.

The receptionist looks completely weirded out.

EXT. SPCA - DAY

Elliot, Leone, and Jayesh are sitting in the loser cruiser, deflated.

\section{JAYESH}

I mean, they must have dogs cark it

all the time. I really don't see

the prob.

Elliot shakes his head.

\author{
ELLIOT \\ (defeated) \\ Let's go tell the truth.
}

Jayesh starts the engine.

EXT. TRINA'S HOUSE - DAY

The unmarked police car is parked up on Trina's street.

Trina takes a seat on the kerb outside her house. She pulls out Victor, and holds him out in front of her face so that she's looking straight into his eyes.

$$
\text { What do you think? }
$$

Trina kisses Victor on his head, which triggers his robotic whinny.

Trina sighs.

TRINA

I know. But we could really help

them out?

Trina strokes Victor's head so that he whinnies again.

TRINA

I know. I know. I love you too. 
INT. UNMARKED POLICE CAR - DAY

The police officers are frowning and squinting at Trina.

MALE POLICE OFFICER

Is she talking to a toy?

FEMALE POLICE OFFICER

Those people have really messed her

up.

The female police officer gets out of the car.

EXT. TRINA'S HOUSE - DAY

The female police officer approaches Trina who whips Victor out of sight.

\section{FEMALE POLICE OFFICER}

Are you OK little lady?

Trina frowns, creeped out.

I think so?

$$
\text { TRINA }
$$

FEMALE POLICE OFFICER

Are there people in your life who

are forcing you do things you don't want to do?

Huh?

TRINA

Trina thinks about the question, and has a realisation.

TRINA

No. I guess you're right. It's me who's making me do things I don't want to do.

The female officer doesn't know what this means, but it makes her concerned.

FEMALE POLICE OFFICER

What kinds of things?

Trina stands up.

TRINA

Being the mum all the time.

FEMALE POLICE OFFICER

What's that? Role play or

something?

Trina ignores the female officer's question. 


$$
\begin{gathered}
\text { TRINA } \\
\text { Sucking on Victor's ear. }
\end{gathered}
$$

The female officer's eyes go wide.

TRINA

I don't wanna do that anymore. I

just want to be free to be me.

Trina marches down the street.

The female officer hurries back to the police car.

INT. LOSER CRUISER - DAY

Jayesh has parked the loser cruiser at the end of old Man Sam's street again.

Elliot's phone rings, and he sinks when he sees it's Mandy.

Elliot is about to answer it, when Trina appears and taps on Elliot's window.

The three of them jump in terror.

Elliot winds down his window, and lets the call go to voicemail.

Trina looks guilty as she makes her confession.

TRINA

I haven't been completely honest with you.

Elliot raises his eyebrows.

ELLIOT

What? Your name isn't Beyoncé?

Trina frowns at Elliot.

TRINA

$$
\text { No, it's Beyoncé. }
$$

Trina bends down and take Victor out of her bag. She shows it to them.

$$
\text { This is Victor. }
$$

They all gawk at Victor.

Did you steal it? 


\section{TRINA}

What?! No. I've had him since I was a baby.

$$
\text { LEONE }
$$

You've had it this whole time?!

Him. And yes.

$$
\text { TRINA }
$$

The three of them look confused.

\section{ELLIOT}

So we can use it...him?

Trina takes a moment.

$$
\text { TRINA }
$$

I'll be getting him back, right?

Elliot grimaces

$$
\text { ELLIOT }
$$

I mean...He's probably going to get buried, but you could sneak back another time and dig him back up?

Trina thinks about this.

$$
\text { OK, then. }
$$

TRINA

The three of them look relieved.

Trina hops into the loser cruiser.

JAYESH

I think there's a bag back there.

And some tomato sauce rolling under

a seat somewhere.

Jayesh drives off and Leone and Trina search for the bag and tomato sauce.

EXT. OLD MAN SAM'S HOUSE - DAY

The loser cruiser pulls up outside Old Man Sam's house.

INT. LOSER CRUISER - DAY

Trina winces as she finishes wrapping Victor up in the bag, and smearing it with sauce. 
EXT. OLD MAN SAM'S HOUSE - DAY

The four of them walk up to Old Man Sam's door. Before any of them can knock, old Man Sam flings the door open.

Old Man Sam sees the Tyche-sized package in Trina's arms, and his eyes fill with tears again.

Trina reluctantly hands the package over.

\section{LEONE}

Don't open it up though. You don't

want to see her in such a state.

Everyone nervously watches old Man Sam handling the package.

ELLIOT

Are you gonna be OK?

Old Man Sam stares down at the package.

OLD MAN SAM

Will you help me give her a proper burial?

Everyone tenses a little bit. Trina is the only one who seems on board.

$$
\text { of course. TRINA }
$$

Old Man Sam lets everyone inside.

EXT. OLD MAN SAM'S BACKYARD - LATER

Elliot is holding a spade in front of a small hole.

Everyone else stands around the hole. Old Man Sam, still

clutching the package, steps forward.

OLD MAN SAM

I hated you with a passion, Tyche.

The four of them look at each other, confused.

OLD MAN SAM

But when Ellen died, you were the

only one who understood my pain.

And now you're gone. And I'm really

gonna miss you.

Old Man Sam kisses the package, and lowers it into the hole.

Trina struggles to contain her sadness at seeing Victor in such a vulnerable position. 
Old Man Sam nods to Elliot who proceeds to fill the hole with dirt.

Old Man Sam starts humming a low, sad song.

Trina bursts into tears. Leone, Jayesh, and Elliot all look at her like she's nuts.

When Elliot is done filling the hole, Old Man Sam stops humming.

$$
\text { OLD MAN SAM }
$$

Thank you.

The four of them are standing around, unsure what to do.

ELLIOT

We should probably get going.

Old Man Sam nods, solemnly.

$$
\begin{aligned}
& \text { ELLIOT } \\
& \text { Again, we're really sorry about } \\
& \text { what happened. } \\
& \text { OLD MAN SAM } \\
& \text { Just don't do it again, OK? }
\end{aligned}
$$

Everyone laughs awkwardly.

$$
\text { We won't. }
$$$$
\text { ELLIOT }
$$

Elliot, Jayesh, Leone and Trina all move towards the house, relieved that it's nearly over, when the faint sound of Victor's robotic whinny can be heard coming from underground.

They all look to Trina, concerned. She flashes them an apologetic expression.

$$
\begin{array}{r}
\text { TRINA } \\
\text { (whispering) }
\end{array}
$$

Shoulda taken the batteries out.

They turn, tentatively to see if old Man Sam has heard.

He has.

Old Man Sam kneels down on the ground and starts digging the dirt.

$$
\text { Tyche! }
$$

$$
\text { OLD MAN SAM }
$$

Old Man Sam turns to the others with hope and desperation.

$$
\text { OLD MAN SAM }
$$

She's still alive! 
Old Man Sam goes back to digging. The sound gets louder. The four of them swarm around Old Man Sam.

$$
\text { I don't think so. }
$$

Leone kneels down and physically tries to stop old Man Sam from digging, but nothing's going to stop him.

JAYESH

I think your grief must be playing

tricks on your head-thoughts.

Desperate, Leone grabs the shovel and lifts it above Old Man Sam's head, as if she's gonna take him out with it.

Elliot eyes Leone, disbelieving.

Old Man Sam's hand touches the rubbish bag. He yanks it out. The four of them step backwards, bracing for impact.

Old Man Sam rips into the bag and pulls out Victor.

He holds it, completely baffled. The toy pony continues to make the horrible sound.

Old Man Sam turns to the Bad News crew who are all cowering in a huddle.

\section{OLD MAN SAM}

What's going on?

Elliot is about to confess, when several POLICE OFFICERS, including the two who have been watching Bad News, barge through the back door and surround the lawn with guns cocked.

$$
\begin{aligned}
& \text { FEMALE POLICE OFFICER } \\
& \text { Everyone stay where you are! }
\end{aligned}
$$

Everyone freezes, terrified.

Get the girl.

FEMALE POLICE OFFICER

A couple of police officers grab hold of Trina and pull her aside.

$$
\begin{gathered}
\text { FEMALE POLICE OFFICER } \\
\text { Who's in charge of this operation? }
\end{gathered}
$$

Everyone but old Man Sam points to Elliot. Elliot is not happy about this.

\section{ELLIOT}

What? Come on guys. We never discussed that. 


$$
\begin{gathered}
\text { LEONE } \\
\text { It was your idea. }
\end{gathered}
$$

Elliot crumbles under the pressure of everyone staring at him. He bursts into tears.

$$
\begin{aligned}
& \text { ELLIOT } \\
& \text { Please don't arrest me. I'm so so } \\
& \text { sorry. I mean, the dog really is } \\
& \text { dead, it just got stolen or } \\
& \text { something. We shouldn't have lied } \\
& \text { about it, but it was our first case } \\
& \text { and we didn't want to botch it. }
\end{aligned}
$$

The police all look very confused.

$$
\begin{aligned}
& \text { FEMALE POLICE OFFICER } \\
& \text { What are you talking about? }
\end{aligned}
$$

Elliot looks confused.

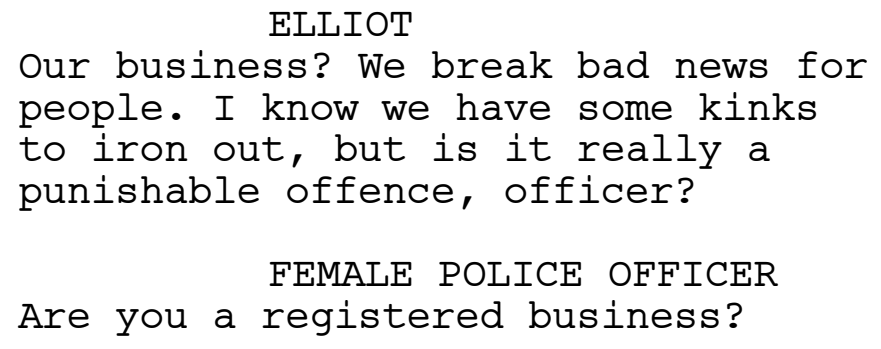

ELLIOT

Our business? We break bad news for people. I know we have some kinks to iron out, but is it really a punishable offence, officer?

FEMALE POLICE OFFICER

Are you a registered business?

Elliot is speechless.

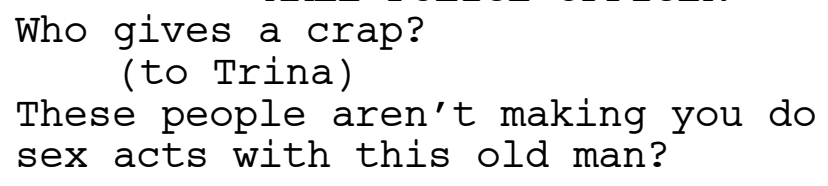

Old Man Sam looks utterly bamboozled.

Ew. No.

TRINA

Old Man Sam looks offended.

FEMALE POLICE OFFICER

Then who's Victor?

Trina points to the dog in Old Man Sam's hand.

FEMALE POLICE OFFICER

You suck that thing's ear?

Trina crosses her arms.

TRINA

Don't be judging me. People can change. 
The female officer nods to the officers guarding Trina. They let her go.

ELLIOT

Why would you think we'd be making

her do sex acts with old Man Sam?

Old Man Sam?

OLD MAN SAM

The female officer pulls out her phone, clicks into

something, and shows it to Elliot - it's his Bad News turned Sex Trafficking ad.

Elliot's face falls.

$$
\text { (to Trina) }
$$

ELLIOT

That's what you meant when I should

check out the ad?

Trina squints at the ad on the female officer's phone.

$$
\text { TRINA }
$$

Oh, yeah. That.

ELLIOT

Why were you not more specific?

\title{
TRINA
}

Don't put this on me.

Trina turns to the female officer.

TRINA

My cousin did that. He musta hacked

into Elliot's account and changed

it to get back at him for stealing

his business idea.

\section{ELLIOT}

I didn't steal it! It's a different business idea.

The female police officer shakes her head, bamboozled and a bit disappointed.

\section{FEMALE POLICE OFFICER \\ Move out troops.}

The female officer leads the team back through Old Man Sam's house.

All is quiet and awkward.

\author{
ELLIOT \\ (to Old Man Sam) \\ Sorry for the commotion.
}


Old Man Sam doesn't respond.

\section{ELLIOT}

We'll let you be alone.

Elliot, Jayesh, and Leone all head towards the house. Trina lingers back.

TRINA

I could take that...

Trina points at Victor.

TRINA

...off your hands if you like?

Elliot whips his head around.

$$
\begin{aligned}
& \text { Tri-- ELLIOT } \\
& \text { (catching himself) } \\
& \text { Beyoncé! }
\end{aligned}
$$

Trina turns, dejected, to Elliot.

TRINA

He's probably just gonna throw it
out!

Elliot glares at her.

She lowers her head, and trudges towards the house with the others.

INT. LOSER CRUISER - DAY

Jayesh drives with Leone in the front passenger seat, and Elliot and Trina in the back.

They sit together in a sombre silence.

But Jayesh can't help himself.

$$
\begin{aligned}
& \text { JAYESH } \\
& \text { That was ruff! }
\end{aligned}
$$

Jayesh looks to the others for approval.

Trina laughs out loud. Jayesh smiles, pleased.

Elliot and Leone shake their heads, but are smiling in a disparaging way.

Elliot gets a notification on his phone. 
ELLIOT

Hey, look - we got our first

feedback.

Leone cranes her neck.

$$
\text { What's it say? }
$$

Elliot reads the feedback.

\section{ELLIOT}

She gave us a neutral face.

The four of them all look surprised and pleased with themselves.

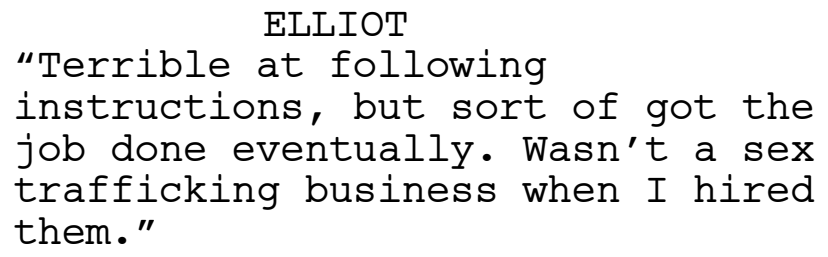

Everyone frowns at this last bit.

ELLIOT

I should really deal with that.

Elliot types frantically into his phone.

TRINA

This is me!

EXT. TRINA'S STREET - NIGHT

The loser cruiser pulls up outside Trina's house.

Trina hops out of the car, and as she is about to close the door says :

TRINA

Thanks for letting me join the

business. It means a lot to me.

Trina closes the door.

INT. LOSER CRUISER - NIGHT

The three of them are left very confused. Jayesh slowly drives off.

LEONE

Did we say she was in the business?

Elliot and Jayesh shake their heads. 
ELLIOT

Deal with it later?

Jayesh and Leone nod.

EXT. TRINA'S HOUSE - NIGHT

Trina waves at the loser cruiser as it disappears - she's happy, but there's an emptiness in her demeanour.

She turns to her house, heads up to her door and is about to go in, but hesitates.

She power-walks down the street.

EXT. OLD MAN SAM'S HOUSE - NIGHT

Trina creeps up to Old Man Sam's house, and stops at the wheelie bin outside his house. She searches through it, but doesn't find what she's looking for.

She creeps up to the house and sees, through the front window, Old Man Sam eating dinner at the dinner table with Victor sitting next to him - a place mat set up especially for him.

Trina is touched by the sight. But when she sees Old Man Sam spoon feed Victor while kissing and cooing over him, she cocks her head and grimaces.

TRINA

So that's what I look like.

Trina puts her hand up on the window.

TRINA

Goodbye, friend.

Old Man Sam sees Trina in the corner of her eye, and Trina quickly darts away.

EXT. RESIDENTIAL STREET - NIGHT

Trina walks down the street, past the beaten-up sports car.

INT. BEATEN-UP SPORTS CAR - NIGHT

A SILHOUETTED FIGURE sits in the driver's seat, talking on their phone.

VOICEMAIL (PHONE)

You've reached BAD NEWS, please

leave a message after the beep. 
The phone beeps.

MALE VOICE
Hi...Uhhh...So...I just found my car all beaten up and I think it must have been someone from my group...I mean from the spiritual healing group I started up. One of them must have found out it's all a sham, so I really need to gain the upperhand, y'know? Before they all find out and go ape shit on me. Can your business do that for me?

\author{
EXT. RESIDENTIAL STREET - NIGHT \\ Trina continues walking, past the state house from the \\ beginning.
}

EXT. STATE HOUSE/ BACKYARD - NIGHT

Magnus leans on a long stick and shines a torch over a small hole he's dug into the ground.

The real Tyche lies in the hole, next to two ripped-off sidemirrors, four hubcaps, and the "HEALBOI" numberplate from the sports car.

Magnus is sniffling when his little sister comes out the back door and stands beside him.

$$
\begin{gathered}
\text { SISTER } \\
\text { What're you doing? }
\end{gathered}
$$

Magnus wipes his eyes.

\title{
MAGNUS
}

Saying goodbye to my best friend.

The sister frowns at the mangled body of Tyche amidst the car parts.

\section{MAGNUS}

She visited me the same time every

day after school. And now she's

gone, and I don't know what I'm gonna do.

The sister puts her arm around Magnus' waist.

\section{SISTER}

I'll be your best friend.

This calms Magnus down.

Magnus salutes Tyche. 


$$
\text { Heel, boy! }{ }^{\text {MAGNUS }}
$$

The sister copies Magnus' salute.

$$
\text { Heel, boy! SISTER }
$$

INT. TRINA'S HOUSE - NIGHT

All is dark, except for the glare coming from a cellphone that lights the face of Russ.

Russ sits on the edge of the couch, listening to a voicemail on his phone with a maniacal grin.

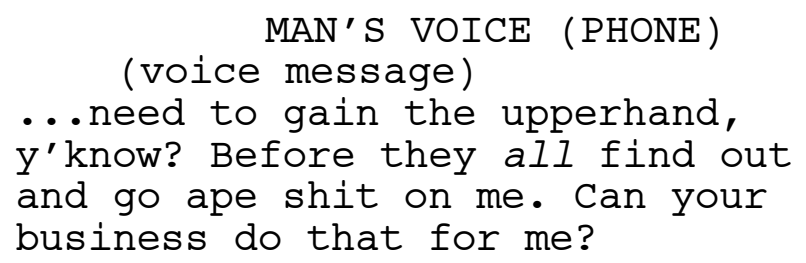

Trina enters, and flicks the light on - causing Russ to squint and cower pathetically.

TRINA

Russ? What are you doing here?

RUSS

Your Dad said I could crash here

for a while.

Trina shakes her head at him.

TRINA

You got Elliot into a lot of shit cos of that ad.

Russ smiles again.

Good.

RUSS

Trina rolls her eyes at him.

TRINA

You need to grow up.

Trina heads upstairs.

When she's out of sight, Russ flicks the light off again and resumes his evil stance. 
INT. TRINA'S HOUSE, UPSTAIRS HALLWAY - NIGHT

Trina creeps up to her sisters' bedroom door which has "Lily" and "Harriet" written on it.

She pushes the door open, and turns the light on - waking her two sisters up.

The three of them have a whispered conversation.

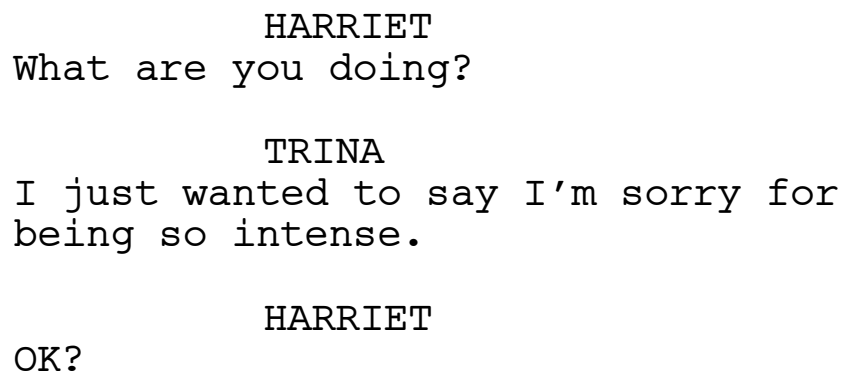

HARRIET

What are you doing?

TRINA

I just wanted to say I'm sorry for being so intense.

OK?

$$
\text { HARRIET }
$$

TRINA

You're meant to say "You're not intense, Trina".

LILY

You are intense, though.

Trina frowns, but then sees that Lily has put the wilted weed into a glass of water that sits on her bedside. She smiles.

TRINA

Good night.

Trina flicks the light off.

End of Episode. 



\section{BAD NEWS AND CHAOS}

My second attempt at writing a serial through the lens of chaos-Bad News-was a much easier experience compared to that of Untold. In part, this was due to the fact that I had more of an idea about the characters and what the story was going to be about, but it was also due to the premise itself being much simpler and more episodic.

I set out to overtly incorporate strange attractors into the narrative structure of this serial by having each of the cases be interconnected without the characters realising it-the characters are embroiled in an ongoing chain of events that appears to them as separate and closed-off events. One person's bad news leads to another person's bad news and so on, in an interlinked chain of cause and effect that means the characters are inadvertently creating their own feedback loop and narrative orbit. Each of the cases is always drawn back to the central conflict of the serial-the push and pull between needing to put an end to something in one's life and the desperation to avoid the confrontation this ending would involve. The intention with this story is that the avoidance-coming from the clients as well as from the members of Bad News-generates more problems than if the characters had just confronted the issues themselves. In this sense, the human flaw of avoiding difficult things in life means that the characters in this serial become stuck in a kind of cycle that allows for things to always be the same, but still have variety. Because of this, Bad News is both continuous and episodic.

Because strange attractors were at the forefront of my mind when writing this serial, I decided that, instead of restricting the feedback loops to the narrative threads of each individual episode, the entire first season would feed back on itself-creating a larger orbit that frames the smaller ones. The first season ends with a case stemming from an incident that began the entire serial but seemed inconsequential when it happened. The main character, Elliot, parks his scooter illegally causing a series of other vehicles to have to park in spaces they're not meant to, resulting in someone dying. The final episode of the season introduces a new case for the business that explains why the man in the car intentionally slammed into the truck, setting off a potential new trajectory for the business that will 
shape the second season (a storyline in which the Bad News characters get in way over their heads with a murder case). In other words, the first season ends in nearly the same place that it begins, but because it is not a complete circle, the second season will have its own distinct narrative trajectory while also maintaining the essence of season one.

Another benefit of interconnecting the narratives in this self-similar and self-generating way, was that when I introduced anything to a narrative-even if it was completely random at first -I could then use that thing as a way to shape the episode or narrative arc. I would work towards paying off the often-random elements that I introduced-therefore giving shape and direction to the episode or overarching storyline. Or sometimes it would happen in reverse, I would write something into the script and realise I could build towards that thing in an interesting way by setting it up earlier on. For example, at the end of the first episode Elliot gets a call from his first Bad News client-a woman who has to tell an old man that his beloved dog was hit by a car while she was taking it for a walk. I originally didn't introduce the storyline for this dog until the end of the episode, but realised that it could be useful, and entertaining, to subtly drop the dog in at the start in a way that made it seem insignificant. So, I had the dog take off on its leash while the woman was walking it past the disorderly scene caused by Elliot parking his scooter illegally. In doing so, my intention was to establish the nature of the show-that random and insignificant events can come into play in a much bigger way further down the line (in a way that reflects sensitive dependence on initial conditions). I then went on to use the death of the dog to generate a storyline in Episode Two in which a teenager acts out because the dog used to visit him every afternoon, giving him a reprieve from his difficult home-life. When the dog stops visiting (due to being killed) the boy lets out his frustration and sadness by smashing a car, which ultimately leads to another case for the Bad News business in which the owner of the car, a fake spiritual healer, wants to use Bad News to come clean about his deception. So, not only do small moments have large consequences for the characters and in the narrative's progression, I also wanted any moment in the serial to have the possibility of functioning as a beginning, middle, and end all at once-in the same way that dynamical systems behave when a strange attractor is present. In this way, because everything has the potential to be significant, an audience can never precisely predict which aspects are going to be important in the narrative. 
The flipside of interconnecting the narrative threads in this way is that when it came to rewriting the serial, it was more difficult to shift things around. Usually when I rewrite a script I'm open to changing whatever needs to be changed in order to make it better, but since everything was so precisely and directly connected in this serial, I found it difficult to make drastic changes. Just as one event or incident in the serial could have big consequences further into the narrative, if I took one of these elements away, or changed it, the entire serial might fall apart; changing one aspect would mean having to change another aspect, which would mean having to change something else and so on. In this sense, the experience of writing Bad News has been similar to that of writing Untold, in that using chaos as a launching pad into both serials meant that I was prioritising structure over the story-albeit to varying degrees.

One major aspect of the story that I did change from one draft to another was the way in which the members of Bad News come together to form the business. In an early draft Elliot, Leone and Barry worked for a business that took people's unwanted junk away, but the leap from taking people's physical junk away to taking people's emotional junk away felt contrived and messy to me. I decided to change it so that Barry, Leone and Jayesh are all living together as hermits because of their shared experiences of accidentally killing someone. This shift meant that these three characters already have an emotional, and domestic, connection before the serial begins, which allows for the serial to explore storylines that more directly tie into the themes of chance, misfortune, and karma. This change to the set-up of the serial demonstrates, again, how simplifying or streamlining particular aspects of the narrative can open a serial up in other ways.

So, whether it has positive or negative effects, using strange attractors as a framework for Bad News has elucidated the narrative connectivity that is inherent in all TV drama narratives. The ripple effect that can happen in both the writing and rewriting of a TV project is something that all serials face. It's just that, by approaching the writing process with chaos in mind, this challenge of writing a serial drama is heightened. The third and final serial that I am presenting is The Chances, which also demonstrates the challenges and rewards of TV's narrative connectivity. 



\title{
THE CHANCES
}

\author{
Written by
}

Alison McLachlan 



\title{
THE CHANCES
}

\author{
$6 \times 1$-hour drama series
}

The Chances is an off-kilter drama that explores people's discordant reactions to an inconceivable coincidence.

When a series of strange events leads Bertha Jessop, an insecure and impressionable 12-year old, to realise that she is one of many Berthas throughout history who all look the same, her life changes forever.

But this change isn't necessarily a good thing, and she-and all the other Berthas throughout history-must grapple with their own sense of reality while navigating delusions, manipulations, mysteries and deceptions, if they're ever going to be fulfilled and find the answers they're all searching for.

Is the coincidence proof of destiny? God? Is there a scientific explanation? Is it simply a random occurrence that has no deeper meaning? Or is there more to the world than meets the eye?

As this darkly comic series unfolds, and as each Bertha's story unravels, the many-layered narrative of the show takes us to vastly different eras and environments that show us that history repeats, but never in exactly the same way. 

CHARACTERS 



\section{BERTHA JESSOP (1999)}

We meet Bertha Jessop when she is 12 years old-insecure, impressionable, and miserable in her home life with her widowed and alcoholic father, and four burly brothers.

When her twin brothers trick her into thinking she's adopted and create a birth certificate to prove it, Bertha-desperate to be part of a loving family-travels to Jamieson (aka Sunny Cliff), the town written on the fake birth certificate, only to discover that while she isn't adopted, she is part of a much stranger, but equally enticing, kind of family.

Initially, Bertha is easily manipulated and brainwashed by her surrogate family-Howard and Bertha Manning - but as she grows older and sees the promise of unlocking the truth of the universe slip away from her, she must overcome her deep-rooted insecurities and paranoia in order to take control of her own life.

\section{BERTHA MANNING (1967)}

Having grown up as an only child to parents who thought they would never be able to have children, Bertha Manning is a spoilt brat who thinks she's more special and gifted than everyone else.

So, when-at the age of 14-she meets Michael who claims that she's his sister's ghost Bertha deludes herself into thinking this is a confirmation that she is invincible and immortal, so she sets out to use her gifts for her own personal gain and becomes a highly successful hitwoman.

At the height of her criminal career, Bertha Manning's delusions of grandeur are put to the test and she tries to start a new, more admirable life, but her villainous persona is never far from the surface, ready to rear its ugly head whenever the need arises.

\section{BERTHA GREIVE (1951)}

Bertha Greive has been brought up in a very religious household-her father died after her mother insisted on using prayer, rather than medicine, to cure his illness. Because of this oversaturation of faith, Bertha Greive has cultivated a rational and scientific world view as an expression of her rebellious and anti-authoritarian nature.

But Bertha Greive's rational way of thinking is upturned at the age of 18 when she learns she's pregnant even though she doesn't remember ever sleeping with anyone, and then coincidentally meets a man-Bertie Nobody-who looks exactly like her. Unable to find a logical way to explain these two incidents, Bertha Greive turns to faith for her answers and becomes convinced of the existence of God. She also believes that her son is the second coming of Christ and does everything in her power to protect him.

While Bertha Greive undergoes a drastic shift in terms of her world-view, her rebelliousness remains a consistent motivator for her actions and is both her strength and her weakness. 


\section{BERTIE NOBODY (1943)}

Bertie Nobody has been brought up by the members of a travelling circus after he was abandoned by his mother as a baby.

While he doesn't like to admit it, his feelings of rejection and abandonment underscore every decision he makes. His desperation to develop a skill that he can use as a circus act, for example, really stems from his desperation to prove to his mother-whoever and wherever she is-that he is worthy of her love.

When he's 26 he has a dream one night that convinces him he has paranormal abilities and that these are what leads him to his lookalikes. From this point on, Bertie struggles to determine what is real and what isn't-the outlook he decides to adopt will either set him free or imprison him.

\section{BERTA LORENZ (1939)}

Bertha is a successful actress in Germany who believes that everything happens for a reason and that only those who are in tune with the universe and its signs are capable of succeeding. So, after receiving what she believes is a sign from the universe, Berta moves to New York City with an American producer who she is having an affair with, leaving behind her husband and two sons.

However, once in the US the reality far from meets her expectations and she is on the cusp of rejecting destiny altogether when she meets BERTHA ANNE, her doppelgänger, who prevents her from committing suicide and sends her life in an unexpected direction.

As Berta continues to face heartbreaks and disappointment she must make a decision as to whether to move back home or stay in the US-a choice that will force her to accept that everything is random and meaningless, or accept that her particular fate is one shrouded in misery. 


\section{SUPPORTING CHARACTERS:}

ANNA SMITH (AKA BERTHA ANNE) (72): After losing her husband of 45 years to suicide and then finding out that she has a terminal illness, Anna Smith-terrified of dying alone-tricks Berta Lorenz into thinking that they are doppelgangers and that fate has brought them together.

BERTHA AGNEW (11): Bertha Agnew is the Bertha of the future, who comes 64 years after Bertha Jessop. She is extremely proud to be deemed a good-luck charm by her parents, who pawn her off to strangers, so when Bertha Jessop turns up and threatens the good thing she has going she has the potential to go down a destructive path.

TRENT JESSOP (52): Trent is Bertha Jessop's father. Trent never really wanted to be a Dad, but sort of just fell into it - five times over. Since Trent's wife died after giving birth to Bertha, he has had to look after his five kids on his own, a task that he is in no way equipped for. Trent has developed a habit of drinking to excess and, while doing the bare minimum to keep his children clothed, fed and sheltered, is incredibly neglectful of his children's emotional needs.

CALLUM JESSOP (14): Bertha Jessop's brother who is constantly bullying and teasing Bertha because she's such an easy target. He is the brains behind the plan to trick Bertha into thinking she's adopted, so when it leads to Bertha's apparent death, Callum is consumed with guilt and turns to superstition as a way to avoid taking responsibility for his actions.

JAKE JESSOP (14): Callum's twin brother who joins in on Callum's bullying and teasing of Bertha but doesn't enjoy it. When Bertha Jessop is presumed dead because of the prank he and his twin brother pulled, his sensitivity and intuition lead him to the belief that Bertha is still alive somewhere.

BRETT JESSOP (18): Bertha Jessop's oldest brother is a jock through and through, but is probably the most responsible of the siblings due to the fact that his father relies on him to take on the responsibilities of a parent.

KYLE JESSOP (16): Kyle is quiet and non-committal-never joining in on his brothers' cruel treatment of Bertha, but also never putting a stop to it either. He basically wants nothing to do with his family, and can't wait to move out of home and never see them again. 
MICHAEL GREIVE (AKA HOWARD) (25-47): Originally called Michael-the brother of Bertha Greive-Howard has spent most of his life dedicated to understanding how the phenomenon of the Berthas occurred, and intends to use his findings to gain the ability to predict long-term future events. His determination and impatience, however, regularly undermine his pursuit and he ends up manipulating events more than he predicts events.

ODETTE (18-50s): Odette grew up in Sunny Cliff (aka Jamieson) and thinks she witnessed Bertha Manning being crushed to death in the landslide of 1967 as a punishment for having a secret affair with Father David when she was 16 . Filled with guilt and dread that her actions had such a mammoth impact on her hometown, Odette comes up with a rumour (that a man named Jamieson predicted something miraculous would happen in 32 years) as a way to give the town hope and purpose. The rumour catches on much faster than she expects and soon it is completely out of her control, and when the day finally arrives 32 years later she struggles to deal with all the pain and confusion that it brings.

FATHER DAVID (40s-70s): One of the oldest, and most respected, members of Sunny Cliff (aka Jamieson), Father David has a dark side that he has managed to conceal from everyone except Odette. When he hears that someone who looks like Bertha Manning has appeared in town, he is forced to revisit his past mistakes.

JUNE MANNING (40s): June is Bertha Manning's mother, who was told she'd never have children before she had Bertha. As a result, June believes Bertha is a miracle and overindulges her even though she knows, deep down, that it is having a detrimental effect on her daughter's character. It is only when June suspects her daughter is gay that she sees it as her duty to finally crack the whip.

MRS. GREIVE (40s): Bertha and Michael Greive's mother puts her religion above anything else, so when she finds out her daughter is pregnant out of wedlock she sends her away to live with her aunt and wants nothing more to do with her.

CIRCUS FOLK: When a group of circus folk discover an abandoned baby they adopt him, name him Bertie Nobody, and bring him up like they're his family. They like to pull lighthearted pranks on Bertie, but when one prank goes horribly wrong they all believe they are being haunted by Bertie's ghost. 
THE CHANCES

Episode Synopses (1-6) 



\section{THE CHANCES}

\section{Episode One: “Bertha Jessop"}

BERTHA JESSOP (12) fantasises about being part of a loving and nurturing family-the complete opposite to her real family-so when her twin brothers manufacture a fake birth certificate and tell her that she's adopted, Bertha Jessop believes it and uses the made-up information to go in search for her made-up birth mum.

A STRANGE MAN shadows Bertha as she travels to the odd town of Jamieson-where all the townspeople are camped out in a park, waiting for a "prediction" to come true. Bertha is devastated to learn that the address that her brothers wrote on the birth certificate was destroyed in a landslide 32 years ago, and when she calls her brothers to tell them, they break the news to her that none of it was real anyway.

While Bertha waits for her brothers to collect her, she overhears a conversation between FATHER DAVID and a townsperson about a woman named Odette-the name of her fake birth mum.

Curious, Bertha tracks down ODETTE CHEN at her ramshackle house on the shoreline, and is stunned when Odette recognises her and knows her name.

Meanwhile, Bertha's brothers are travelling to Jamieson when they are intercepted and misdirected by the strange man who has been shadowing Bertha.

Bertha is spooked to learn that she is identical to a girl name Bertha Manning who was killed in the landslide 32 years ago, and Bertha runs away when Odette tries to convince her to pretend to the townspeople that she's Bertha Manning's ghost as part of the "prediction" which-Odette reveals-she made up after the landslide to give the town hope again.

While Bertha waits for her brothers to pick her up, the strange man introduces himself as Howard and convinces Bertha to go home with him by telling her that she is one of several Berthas throughout history, who all look alike and have other strange similarities, and that she might be the key to solving the mystery of the universe.

Meanwhile, Odette wakes the townspeople up to tell her that the prediction is happeningBertha Manning has returned from the dead-only to learn that no one ever actually believed the prediction was real. Odette is rudely dismissed by everyone but Father David, who is intensely shaken upon hearing Odette talk about Bertha Manning returning from the dead.

While another landslide occurs in Jamieson, in the exact same place as last time, blocking Bertha's brothers from being able to get into the town, Bertha is warmly welcomed into Howard's home and is stunned when she is introduced to Howard's wife - the supposedly dead BERTHA MANNING. 


\section{THE CHANCES}

\section{Episode Two: "Bertha Manning"}

Sunny Cliff (aka Jamieson), 1967: A young Bertha Manning brags to Odette that she plans on using her gifts to get her crush, Father David, to marry her. But when JUNE MANNING, Bertha Manning's overly-adoring mother, observes Odette and Bertha Manning together she suspects that her daughter might be gay and so sets out find her a boyfriend.

Jamieson (aka Sunny Cliff), 1999: When police ask the townspeople for information about the now-missing Bertha Jessop who, to the befuddlement of the older members of town, looks exactly like Bertha Manning, Father David discovers that Odette has drowned herself because she believed herself to be cursed.

Meanwhile, Betha Jessop is treated by Howard and Bertha Manning like she's part of the loving family she always dreamed of, while the Jessops are told by police to be prepared for the worst after they find some of Bertha Jessop's belongings amidst the rubble of the landslide.

Sunny Cliff, 1967: When Michael (25) turns up in the town after escaping a cruise ship on which all the passengers were infected with a deadly rash, June Manning takes Michael in, seeing him as a potential suitor for her daughter who, unbeknownst to June, has made plans to meet up with, and woo, Father David that night.

Jamieson, 1999: Bertha Jessop's idyllic new life is overturned when she learns that Howard has been stalking her since she was born. And when Bertha Jessop tries to escape the property Howard and Bertha Manning tranquilize and brainwash her until she promises to stay with them.

Sunny Cliff, 1967: After Michael is introduced to Bertha Manning he reveals to her that she looks just like his sister who died years before from the same rash that broke out on board the cruise ship. Michael is convinced that Bertha is his sister's ghost and that he has been sent to rescue her. Extremely unnerved by this revelation, Bertha Manning goes to Father David's house for advice, but is horrified when she discovers that he and Odette are having an affair.

Jamieson, 1999: After learning that Howard's sister was one of the Berthas, Bertha Jessop searches for more answers and when she discovers a "wanted for murder" poster that has a photo of Bertha Manning on it, Howard distracts her by making an impossible prediction.

Sunny Cliff, 1967: As a hard rain falls down on Sunny Cliff, Michael convinces Bertha Manning to run away with him after he overhears June calling a psychologist to have Bertha Manning admitted to a psych ward to cure her of being gay.

As Michael and Bertha Manning are driving out of Sunny Cliff they narrowly escape the landslide that kills Bertha Manning's parents, while Odette believes she witnesses Michael and Bertha Manning being crushed to death. 


\section{THE CHANCES}

\section{Episode Three: "Bertha Greive"}

Australia, 1951: When BERTHA GREIVE (18) contracts a rash, she goes to the doctor in secret-after her religious mother insists on curing it with prayer rather than medicine-and learns that she is pregnant even though she insists she is a virgin.

While avoiding telling her family, Bertha Greive extracts a figurine inscribed with the name "Bertie" on it, from some dried cement. When she later learns that the body of a man named "Bertie" has washed ashore in a coffin after being missing for 8 years, Bertha tracks down Bertie's relatives who are spooked by her resemblance to Bertie.

When Bertha Greive finally tells her mum about her pregnancy, she is sent away to live with her aunt, and Bertha's brother, Michael, is told that her sister died from the rash that she contracted.

Bertha Greive is living at her aunt's where she becomes convinced that her pregnancy was an immaculate conception, and that it has something to do with Bertie who she learns was abandoned by his mother as a baby.

New Zealand, 1967: Bertha Manning and Michael are living a hard life on the streets until Michael abandons her when he gets news that his sister is actually alive and looking for him.

While fending for herself, Bertha Manning discovers, due to her sense of invincibility and immortality, that she is a very good at killing people for money, and soon becomes a successful hit woman.

Meanwhile, Jamieson struggles to deal with the effects of the landslide, and Odette, plagued with guilt, starts the rumour about Jamieson's prediction.

Jamieson, 2003: Four years have gone by, and Bertha Jessop-now 17-still lives with Howard and Bertha Manning, who have made no progress in finding an answer to the mystery of the Berthas, leading Bertha Jessop to develop compulsive tics as a way to manage her paranoia and anxiety.

Bertha Jessop makes a discovery that proves that keeping her captive is detrimental to Howard and Bertha's investigation-they've been manipulating and controlling events, rather than letting them happen naturally-and so she convinces Howard and Bertha to let her go.

As Bertha Jessop ventures out of the property for the first time in four years, Howard makes a new discovery that he believes proves there are parallel universes. 


\section{THE CHANCES}

\section{Episode Four: "Bertie Nobody"}

Australia, 1943: BERTIE NOBODY (24)-abandoned by his mother and adopted by a travelling circus-is desperate to cultivate a skill that he can turn into a circus act. He becomes convinced that he is psychic when he has a dream about a figurine stuck in a patch of wet cement in the park that he was abandoned in.

Thinking his dream might lead him to the identity of his mother, Bertie doesn't find the figurine but convinces himself that he is the one that has to plant it.

Bertie waits for something to happen with the figurine until he is arrested for robbing the figurine from a toy store and he is taken to police station where he has an eerie meeting with BERTA LORENZ who thinks Bertie is her estranged son.

At first, Bertie thinks his circus family are pranking him, so he gets back at them with his own prank, but it has disastrous repercussions, and he eventually realises the interaction with Berta was real and that his dream led him to it.

His circus family grow sick of Bertie's ramblings so trick him into thinking that they've made a powder that can transport him into parallel worlds. Bertie consumes the powder one night, and the next day is nowhere to be seen.

Australia, 1958: Bertha Greive is living with her Aunt and six-year old son, who has been brought up thinking Bertha is his sister, when Bertha becomes convinced that her son is the second coming of Christ and is in great danger.

Bertha Greive kidnaps her own son in an attempt to keep him safe, but her Aunt alerts the authorities and Bertha Greive is forced to leave her son at a convent before being sent to jail.

New Zealand, 1968: Bertha Manning is at the height of her success as a hit-woman when she botches a case after unexpectedly encountering Howard (AKA Michael) who tells her that his sister is still alive and that she is not immortal, but there are other Berthas throughout history.

Howard and Bertha Manning get married and secretly move back to Jamieson where they attempt to uncover the secrets of the universe.

Jamieson, 2003: When Howard dies from his attempt to access a parallel universe, he leaves Bertha Jessop with the choice to either kill herself in order to potentially access the parallel universe or continue living her life without ever knowing whether the parallel universe exists.

Bertha Jessop shocks her family when she returns home after four years of them thinking she was dead, while Bertha Manning stays behind-having grown terrified of ever leaving the house. 


\section{THE CHANCES}

\section{Episode Five: "Berta Lorenz"}

Germany, 1939: After receiving what she believes is a sign from the universe, BERTHA LORENZ (42)-a famous German actress-moves to the US with a producer who she is having an affair with, leaving her husband and children behind in totalitarian Germany.

Once in the US, the producer promptly abandons Berta and she becomes suicidal as she struggles to fend for herself in an alien country.

Just as she is about to jump from the bridge she visits every day for weeks, an OLD WOMAN stops her, and tells her that her name is Bertha Anne, and that Berta looks exactly like she did at her age and was once approached by another Bertha who claimed the same thing.

After Berta sees pictures of Bertha Anne and the Bertha before her, Berta-deducing that this must be her purpose in life-dedicates her days to looking after Bertha Anne who is dying of a terminal illness.

As Bertha Anne draws closer to her death, Berta uncovers a devastating truth about Bertha Anne that challenges her faith.

Australia, 1943: Bertie Nobody wakes up in what appears to be a parallel universe where he discovers he has the gift of talking to the dead, and uses it to get a job at the circus.

At the height of his fame, Bertie is stripped of his gift after he encounters the ghost of a woman named Anna Smith who tells him that he is in the wrong place.

Bertie convinces himself that his life as a successful medium was a premonition, so sets up a stall and pretends he can talk to dead people, but his customers are more interested in the webbed fingers and feet that he has developed and he lives his life as a freak show until one day he wakes from a dream and finds himself in a coffin, washed up on shore, and is told by Bertha Greive that he has been missing for eight years.

Australia, 1967: When Bertha Greive is released from prison she secretly watches her son live his life from afar, until she witnesses him being bullied and tries to step in but is met with fear and loathing. Full of regret, Bertha Greive returns to her home only to learn that her mother is living alone with dementia and her brother, Michael, was allegedly killed in a landslide in New Zealand.

Jamieson, 1987: Having calculated the exact date that Bertha Jessop will be born, Bertha Manning and Howard discretely travel to Hamilton, NZ to watch the birth, but inadvertently kill Bertha Jessop's mother, and are forced to cover their tracks.

Hamilton, 2003: Bertha Jessop struggles to adjust being home after four years of everyone thinking she was dead, but when she opens up about the truth about her lookalikes, Bertha is inundated with calls from people who have had similar experiences but haven't come forward for fear of being thought to be crazy 


\section{THE CHANCES}

\section{Episode Six: "Bertha Agnew"}

New York City, 1935: ANNA SMITH's (72) life is turned upside down when she gets a call from police telling her that her husband of 45 years has jumped off a bridge and killed himself.

Anna struggles to come to terms with her new life as a widow, and routinely visits the bridge her husband jumped off where she notices another frequent visitor, a suicidal woman.

After learning that the woman is the famous German actress, Berta Lorenz, Anna suffers a fall and ends up in hospital where she is told that she has an incurable cancer.

Terrified of dying alone, Anna concocts a plan; she cuts out photographs of Berta from magazines and manufactures photographs that will convince Berta into thinking they are doppelgangers.

New York City, 1939: Berta Lorenz is furious to find out that Anna lied to her about being her doppelgänger, but finds it within herself to help Anna overcome her fear of dying, and is at her bedside when she passes away.

When a nurse walks in on the scene she suspects Berta Lorenz of aiding Anna's death. The police take Berta into the police station for questioning where she bumps into Bertie Nobody who looks just like she did at his age.

After trying to find meaning in her meeting with Bertie, Berta Lorenz deduces that it is nothing more than a coincidence, and returns to Germany where she must convince her family to forgive her for leaving.

Australia, 1951: When Bertie Nobody tells police that he believes he has been travelling between parallel universes for the past eight years, the police-for entertainment's saketell him they'll lock him up in a psych ward unless he can prove the parallel worlds exist.

Bertie returns to his circus family to retrieve the powder, but they don't seem to be able to see him so Bertie makes his own powder that unintentionally kills the police officer who ingests it.

Bertie is locked up in prison for murder but is visited by the dead police officer who tells him that he's sorry for not believing his claim about there being parallel universes.

New Zealand, 1967: Bertha Greive follows a hunch that her brother is not actually dead and travels to New Zealand to track him down but, not knowing what he looks like, returns to Australia after bumping into her brother without realising it.

When Howard sees a missing person poster with his face on it and his sister's details, he abandons Bertha Manning and travels back home where he reunites with his sister and 
learns about the Bertha lookalikes, and sets out on his path to uncover the scientific explanation for it.

Meanwhile, Bertha Greive is visited by a deplorable man who claims to be her son's father, but Bertha Greive refuses to accept that her son has a father and joins the convent where she spends the rest of her life.

Hamilton, 1999: While Bertha Manning prepares the home for Bertha Jessop's arrival, Howard shadows Bertha Jessop on the days leading up to her arrival in Jamieson but, growing impatient, he interferes with fate and subliminally plants the idea into Callum's head that he should trick Bertha Jessop into thinking that she's adopted.

Jamieson, 2003: Bertha Manning overcomes her agoraphobia in order to reveal herself to her hometown-which has turned into a thriving tourist destination since the second landslide occurred-and makes amends with Father David before murdering him.

Hamilton, 2003: Using the trends from all the newly emerged cases of doppelgangers, Bertha Manning is able to come up with a formula that gives her the ability to predict future events, just as Howard had set out to do.

Bertha Manning uses the formula to live out the rest of her life in exactly the way she wants-having her own family and giving her children the childhood she always wantedbut because her life lacks spontaneity and unexpectedness she becomes incredibly unhappy, and as the day approaches when the new Bertha is meant to arrive she struggles to decide whether to travel to meet her or not.

2063: BERTHA AGNEW (11) is considered by her gambling-addicted parents to be a goodluck charm and is sold off to strangers who want to win big. But Bertha Agnew's good-luck streak is ended when Bertha Jessop, now an old woman, turns up.

Bertha Agnew steals money from Bertha Jessop to give to her parents to make up for her good-luck streak coming to an end, and Bertha Jessop uses the encounter to bond with Bertha Agnew, before deciding against telling her about the Berthas; she breaks the cycle. 



\section{THE CHANCES}

Scripts (Episode One \& Episode Two) 

THE CHANCES

Episode One:

"Bertha Jessop"

Written by

Alison McLachlan 

EXT. COASTAL TOWN DAIRY (1999) - NIGHT

A full moon illuminates a blackboard easel displayed outside a shabby dairy in a coastal town.

The blackboard has "4 sleeps to go!" written on it in chalk.

Someone smudges the "4" with their finger, and replaces it with a "3".

INT. JESSOP HOUSE, BEDROOM - NIGHT

The moonlight faintly illuminates a dark, cluttered room, crammed with two bunk beds. The ladder from one bunk bed has been removed and placed across the two top beds, like a bridge.

JAKE and CALLUM (both 15 - fraternal twin brothers) share one bunk bed, and are chatting and laughing loudly with no consideration for their sister - BERTHA JESSOP (12 - plump, and uncomfortable in her own skin) - who is struggling to sleep in the top bunk bed opposite them.

KYLE (17) is snoring loudly on the bottom bunk, oblivious.

Jake pushes his feet up against the bottom of Callum's mattress, causing Callum to involuntarily bob up and down on his bed.

Bertha sighs, and sits up.

Callum and Jake stop their scuffling, and watch Bertha closely.

Bertha swings her legs over the side of her bed, which causes the bunk to creak. She goes to put her foot on the ladder that isn't there, and falls to the ground with a thud.

Callum suppresses his laughter while Jake looks a little bit guilty. Bertha, determined to not give them what they want, picks herself up and walks to the door.

Just as she's about to leave, Callum flicks his lamp on and exclaims, exaggeratedly.

\section{CALLUM \\ What have you done, Bertha?!}

Bertha turns to her brothers, notices the ladder resting across the top bunk beds, and frowns, confused.

\section{CALLUM}

Don't you know it's bad luck to walk under ladders?

Bertha stares at the ladder that she just walked under, dismayed. 
I hate you!

BERTHA

Bertha storms out of the room.

Callum and Jake descend into fits of laughter.

CALLUM

She's...so...stupid!

INT. JESSOP HOUSE, HALLWAY - NIGHT

Bertha squints at the brightness of the hallway light, and trudges towards the bathroom across worn and filthy carpet.

INT. JESSOP HOUSE, BATHROOM - NIGHT

Bertha flicks the light on.

Nearly every surface of the bathroom is covered in grime. Bertha is blind to it.

Bertha slips her pyjama pants down and waddles over to the toilet. She puts the yellowed toilet seat down, and takes a seat.

Bertha pees, relieved. But when she drops her gaze towards the floor, she stops mid-stream, mortified. She stares at a browny-red stain that has emerged in the gusset of her undies.

Bertha squeezes her eyes shut, and quickly finishes peeing.

Bertha breaks off a bit of toilet paper, and wipes herself. She briefly inspects the brown, congealed blood on the toilet paper, before scrunching it up and dismissively throwing it into the toilet bowl. She flushes it away.

After washing her hands in the slimy basin, she quickly peeks into the cabinet underneath the sink. All that's there are empty bottles of shampoo, and rusted cans of deodorant and shaving cream.

Bertha grabs a bit of toilet paper, and lines the gusset of her undies with it.

INT. JESSOP HOUSE, HALLWAY - NIGHT

Bertha can hear Jake and Callum continuing to scrap in the bedroom.

She heads to the living room, lit by the intermittent glow of a TV screen. 
INT. JESSOP HOUSE, LIVING ROOM - NIGHT

Bertha hovers timidly in the door frame.

In the far corner of the lounge, the eldest brother, BRETT (19), is sleeping on a mattress in an adjoining nook - his bedroom.

Bertha's dad, TRENT (52), is drunk and coma'd out on the floor in front of the TV. An empty wine glass and a halfeaten packet of chips lies next to him.

A trashy news show is playing on TV:

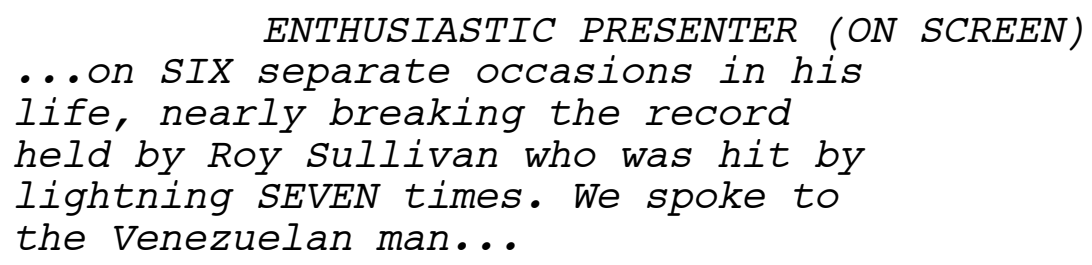

The VENEZUELAN MAN is being interviewed from his hospital bed. He speaks in Spanish, but a TRANSLATOR translates:

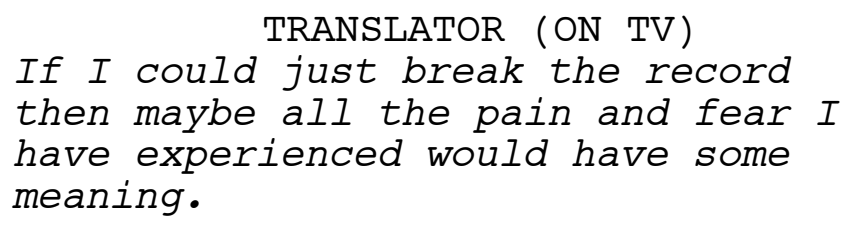

Bertha steps into the lounge, nervous.

\section{Dad?}

BERTHA

Trent doesn't move, but he snores heavily.

Bertha steps closer to him, and gives his limp body a nudge.

Dad.

$$
\text { BERTHA }
$$

Still, Trent doesn't budge. Bertha smells something gross in the air, then steps back disgusted, but not all that surprised.

Her dad has peed himself.

She heads into the adjoining kitchen.

INT. JESSOP HOUSE, KITCHEN - NIGHT

The bench is full of dirty dishes, and old scraps of food. Bertha grabs a soiled tea towel that is hanging on the oven handle. 
INT. JESSOP HOUSE, LIVING ROOM - NIGHT

Bertha sneaks up to her dad again, tea towel at the ready.

She puts it down at her feet, then reaches for the packet of chips and pours the rest of them into the tea towel. She wraps the chips up, and heads back to the bedroom.

INT. JESSOP HOUSE, BEDROOM - NIGHT

Bertha creeps into the bedroom with her package of chips concealed beneath her pyjama top.

Callum pouts at Bertha, tauntingly.

$$
\begin{gathered}
\text { CALLUM } \\
\text { (phoney) } \\
\text { We're sorry Bertha. }
\end{gathered}
$$

Bertha ignores him. She discreetly places the chips on the bed, before putting the ladder back in place and clambering up it.

\section{CALLUM}

Maybe it'll bring you good luck.

Bertha lies down, and turns to face the wall.

\section{CALLUM}

Since you're already so fat and ugly.

Bertha stifles tears.

Jake, annoyed with his brother, pushes his feet up underneath Callum again, nearly causing him to tumble off the bed.

\section{Dick.}

CALLUM

Callum steadies himself, and Jake looks up at Bertha's bed.

JAKE

You OK, Berth?

Bertha is quiet for a moment, and Jake looks worried.

Mhmm.

BERTHA

Jake looks relieved, he turns over to finally go to sleep.

INT. JESSOP HOUSE, BEDROOM - NIGHT (LATER)

All three brothers are now asleep, and snoring loudly. 
Bertha is still awake, and anxiously scribbling into her notebook.

The tea towel sits next to her pillow - now only encrusted with chip crumbs.

INT. JESSOP HOUSE, LIVING ROOM - MORNING

Bertha is in her school uniform and sits, still as a post, on the couch.

Brett, Kyle and Jake mill about, getting ready for school and roughing each other up in the process.

Bertha closely watches her dad, who is in the kitchen. He is dressed in high-vis gear for his rubbish collection job, and is vacantly stirring milk into his instant coffee - his eyes are bloodshot and hands shaky from his night of heavy drinking.

Jake notices Callum is missing, and leaves the room.

INT. JESSOP HOUSE, BEDROOM - MORNING

Jake enters the messy bedroom - the curtains are still

closed, wet towels are lumped on the carpet, and the beds are all unmade.

Callum has found Bertha's notebook, and reads it - grinning. The cover has "BERTHA JESSOP: TOP SECRET" written on it.

\section{CALLUM}

“Fantasy family - point one hundred and fifteen: We eat meals at a real table and tell each other about our days."

Callum cackles with glee. Jake frowns.

Callum flashes Jake a look of pure delight.

\section{CALLUM}

I have an awesome idea.

INT. JESSOP HOUSE, LIVING ROOM - MORNING

Bertha is still sitting on the couch, apprehensive.

Jake and Callum come back into the lounge, and Callum smirks at Bertha. The four brothers all eventually stumble out the door, grunting goodbyes as they go.

Bertha pulls herself up and leans in the doorway to the kitchen where Trent is. 
Dad?

BERTHA

Trent finishes off his coffee in one gulp.

$\mathrm{Mm}$ ?

TRENT

Bertha, embarrassed, glances down and speaks in a hushed voice.

$$
\text { BERTHA }
$$

I need some sanitary items.

Oh.

TRENT

Trent awkwardly takes a sip of his coffee even though there's none left.

TRENT

I don't really know about all that stuff.

Bertha's face drops.

$$
\begin{aligned}
& \text { TRENT } \\
& \text { Maybe go to your school nurse or } \\
& \text { something? }
\end{aligned}
$$

Bertha nods, humiliated.

She grabs her school bag from the lounge, bows her head, and rushes out the door.

EXT. JESSOP HOUSE - DAY

When Bertha reaches the end of the overgrown driveway, she pulls out her jersey from her bag, and self-consciously ties it around her waist.

EXT. HIGH SCHOOL - DAY

The school is abuzz with STUDENTS chit-chatting and fluttering about before school.

EXT. HIGH SCHOOL OFFICE - DAY

Bertha stands near the entrance to the school's office, frozen in fear. She peers into an adjoining sickbay, where a GIRL is holding an ice-pack to the side of her head.

STUDENTS and TEACHERS come in and out of the building, avoiding Bertha but sometimes bumping into her. 
The SCHOOL RECEPTIONIST is behind the desk, and ends a phone call when she sees Bertha standing there.

Ah! Ms. Jessop!

RECEPTIONIST

Bertha glances down, self-conscious.

RECEPTIONIST

A quick word?

The receptionist gestures for Bertha to approach her desk.

Bertha nervously approaches the desk, and the receptionist lowers her voice.

\author{
RECEPTIONIST \\ Your fees for the year 9 camping \\ trip tomorrow haven't been paid.
}

Bertha, mortified, subtly glances around to see if anyone in the vicinity has heard this.

\title{
RECEPTIONIST
}

If we don't receive the fifty

dollars by tomorrow, then we'll

have to find something else for you

to do at school. OK, hun?

Bertha nods, then stares down at the carpet.

\section{RECEPTIONIST \\ Was there something else you were here for?}

Bertha shakes her head, and scurries out of the office.

INT. CLASSROOM - DAY

Bertha is sitting at a desk, pretending to listen to her maths teacher drone on, when she sees the STUDENT sitting next to her reach into her schoolbag and pull out a pen from a side pocket.

Bertha notices a sanitary pad that now sticks out of the sidepocket, and her eyes go wide. Bertha continues to stare at the pad, trying to figure out how she could swipe it.

She slumps her upperbody on her desk, facing the schoolbag, then drops her arm so that it dangles near the girl's bag.

Bertha tenses as she extracts the pad as discreetly as possible, then shoves it quickly into her pocket.

Bertha tries to remain calm despite the adrenaline coursing through her body. 


\section{Miss!}

MALE STUDENT (O.S.)

The male student sitting behind Bertha has his hand up, and everyone turns to him. The TEACHER looks annoyed at the interruption.

\section{Yes?}

\section{TEACHER}

MALE STUDENT

Big Bertha just stole something from Aubrey's bag.

Bertha looks mortified as everyone's eyes fall on her.

$$
\text { No, I didn't. }
$$

BERTHA

Aubrey picks up her bag up and sifts through it to see what's missing.

MALE STUDENT

$$
\text { I saw you! }
$$

The teacher sighs.

\section{TEACHER}

Bertha. If you've stolen something

please give it back to Aubrey

immediately.

Bertha stands up.

$$
\text { I didn't. BERTHA }
$$

Bertha grabs her school bag and runs out of the classroom to the laughs and sniggers of her peers.

INT. SCHOOL BATHROOM - DAY

Bertha enters a toilet cubicle and breathes a sigh of relief as she closes the door behind her.

She takes the pad out of the packet, and inspects it like she's never seen one in her life.

She pulls her skirt up, and pulls down her undies.

EXT. SMALL-TOWN DAIRY - NIGHT

Moonlight illuminates the blackboard, displayed outside the small-town dairy. 
The blackboard still has "3 sleeps to go!" written on it in chalk.

Someone smudges the "3" with their finger, and replaces it with a "2".

INT. JESSOP HOUSE, LIVING ROOM - NIGHT

Bertha sits on the couch, watching a family sitcom on TV while counting out coins that she's poured out of a jar.

When she's done counting she tallies up the total amount and writes it down - it only comes to $\$ 25.25$.

Bertha sinks in her seat when Trent, Kyle and Brett come home. Kyle carries a packet of fish and chips, and Trent has a cask of wine cradled in his arm.

Bertha quickly brushes the coins back into the jar, but Trent catches her.

TRENT

What are you doing?

Kyle and Brett take seats on either side of Bertha - severely cramping her style.

Trent marches over to Bertha.

Bertha looks at Trent, guiltily.

BERTHA

There's this school camp tomorrow

that I need fifty dollars for.

Trent takes the coin jar from her.

TRENT

Fifty dollars? Where're they taking

you? Buckingham Palace?

Bertha glances down.

BERTHA

I showed you the permission slip

ages ago.

Trent shakes the jar.

TRENT

This money's for bills.

Bertha watches Trent take the jar back into the kitchen and place it back on top of the fridge.

He pours himself a glass of wine from his cask, and downs it in one go. 
Bertha sinks back into her seat, miserable.

Kyle and Brett rip open the fish chips, and demolish them. BRETT Callum! Jake! Dinner!

INT. JESSOP HOUSE, BEDROOM - NIGHT

Callum and Jake are sitting on the bedroom floor - they ignore Brett's call.

Callum, tongue poked out in concentration, is meticulously tracing something onto a bit of paper.

Jake watches on, impressed but hesitant.

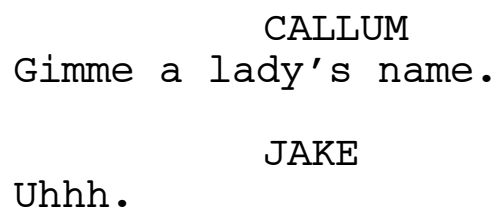

\section{CALLUM}

That'd be funny, but I don't reckon

she's dumb enough to think she's Asian.

Callum sees a bag under the bed branded with "Wilson". Callum writes down Wilson.

$$
\text { Is this too mean? }
$$

Callum shakes his head.

\section{CALLUM}

She should be honoured that we're putting so much effort into it. 
Callum concentrates hard as he writes down what he is saying. CALLUM

D.O.B: Seventeenth of August...

Callum does some calculations on his fingers.

CALLUM

1951. Place of Birth?

Jake doesn't say anything.

Callum eyes a calendar that hangs on the wall - the 20 th of July is circled.

\title{
CALLUM \\ Twenty ...
}

Callum spots a cluster of old tomato seeds encrusted on the carpet.

\section{Seed Street...}

CALLUM

Callum writes this down. Jake frowns.

\author{
JAKE \\ I don't think they put full \\ addresses on them.
}

Callum pauses, regretful.
CALLUM
She won't know that. Name of a
town?

Jake sees an empty bottle of Jameson Whiskey on the dressing table. He speaks reluctantly.

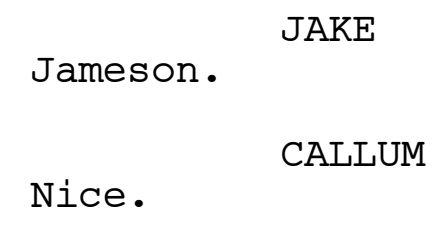

\section{Nice.}

Callum writes this down, then proudly holds up the piece of paper.

We see now that it is a fairly convincing counterfeit birth certificate.

\section{JAKE}

Who knew you had such good penmanship?

Callum smiles, excited. 
INT. JESSOP HOUSE, LIVING ROOM - NIGHT

Trent has changed the TV channel from Bertha's family sitcom to a rugby game.

Most of the fish and chips have been devoured.

Jake and Callum poke their heads into the lounge - grave expressions on their faces.

CALLUM

(whispering)

\section{Bertha.}

Bertha looks at the them, confused.

CALLUM

Can we have a word with you?

Bertha frowns. She looks to Jake for an explanation.

JAKE

It's real important.

Bertha hesitantly follows them into the hallway. Callum conceals a grin.

INT. JESSOP HOUSE, BEDROOM - NIGHT

Bertha stands in the doorway, uncomfortable.

CALLUM

Take a seat.

Bertha doesn't move until Jake nods at her encouragingly.

BERTHA

Why're you acting so weird?

Callum kneels down in front of her, and Jake stands behind him.

\section{CALLUM}

We've been thinking about telling

you this for ages, but we were

never sure if it was the right

time.

Callum looks to Jake to help him out. He does so, reluctantly at first, but gradually gets pulled into the charade.

\section{JAKE}

Mum and Dad made us swear we'd

never tell you.

Bertha looks intrigued. 


\section{CALLUM}

But it doesn't feel right keeping

the secret from you now that you're getting older. We're family, you know?

\section{JAKE}

And we know we're not always very

nice to you, but we do love you.

Callum nods, agreeing. Bertha looks really spooked.

$$
\text { Are you ready? }
$$

Bertha nods.

\section{JAKE}

We told you we love you, right?

BERTHA
Yes! What is it?

Callum lowers his voice.

CALLUM

You're adopted.

Bertha is frozen, almost believing it - then she scoffs.

$$
\text { BERTHA }
$$

Shut up.

\section{CALLUM}

We know it sounds likes a prank, but it's real.

Bertha looks into Jake and Callum's eyes - they don't falter.

$$
\text { CALLUM }
$$

And we have this to prove it.

Callum reaches up to his bunk bed, and pulls out the birth certificate from under his pillow.

He hands it over to Bertha who silently reads it - awed.

She looks Jake and Callum up and down - they're tall and lanky, and nothing like her.

$$
\text { JAKE }
$$

Mum really really wanted a girl, and adopting was the only way to guarantee it.

Callum nods - impressed with Jake's lying. 


\section{CALLUM}

We just thought you should know.

\section{JAKE}

Promise you won't tell dad we told

you.

Bertha is silent - head swimming with the possibilities.

I promise.

$$
\text { BERTHA }
$$

Callum pats Bertha on the head before he and Jake leave the room.

Bertha is stunned. She reads over the birth certificate, hints of joy and hope creeping in.

INT. JESSOP HOUSE, HALLWAY - NIGHT

Jake and Callum silently congratulate each other.

INT. JESSOP HOUSE, LOUNGE - NIGHT

Bertha sits on the couch watching TV with her family sprawled slovenly around her.

The remains of the fish and chip papers are spread out in front of them.

Trent is drunk again, and lying on the floor in front of the TV. He belches loudly.

Bertha surveys her family in disgust.

EXT. SCHOOL CARPARK - DAY

A bus-load of noisy YEAR NINE students are driven out of the school.

INT. LIBRARY - DAY

The LIBRARIAN keeps her eye on Bertha who is sitting at a table with her science text book open.

She copies down an equation until she sees the librarian disappear into a back room.

Bertha scurries over to the library's one computer, takes a seat and logs on to it.

A poster is displayed on the wall next to her that says "BE Y2K WISE!" 
Bertha discreetly pulls out the birth certificate, and types the address on it into a web browser.

The search returns a swathe of random information - nothing that exactly matches the address - but an old news article about a landslide in a town called Jamieson catches her eye.

Bertha clicks into it and is excited to see the words "Seed Road" and "Odette Chen" crop up in the article.

Bertha brings up a new window, and searches the internet for "bus to Jamieson", when the librarian returns and sees Bertha on the computer.

\section{LIBRARIAN}

Did I give you permission, young lady?

Bertha fumbles as she logs out of the computer, and scurries back to her table.

EXT. SCHOOL QUAD - DAY

The quad is empty and quiet until the bell rings and swarms of noisy students pour out of the surrounding classrooms.

Bertha appears from the nearby library. She lowers her head, walks through the busy quad towards the entrance to the school.

EXT. SCHOOL ENTRANCE - DAY

Bertha runs ungracefully out of the school.

EXT. JESSOP HOUSE - DAY

Bertha makes her way up the driveway to her house.

INT. JESSOP HOUSE, KITCHEN - DAY

Bertha enters the house, swipes the jar of money from the top of the fridge, and puts it in her schoolbag.

INT. JESSOP HOUSE, BEDROOM - DAY

Bertha changes out of her uniform and into some regular clothes that she retrieves from the floor.

She puts a few other items of clothing into her bag before leaving. 
EXT. JESSOP HOUSE - DAY

When Bertha gets to the end of the driveway, she turns and looks at her depressing rundown home with its peeling paint and overgrown lawns.

She continues on down the street.

EXT. BUS STOP - DAY

An odd mixture of PASSENGERS hovers around a bus, waiting to be let on.

A STRANGE MAN (47) lingers outside the bus-stop toilet.

INT. BUS-STOP TOILETS - DAY

Bertha pees with her pants around her ankles, revealing the soiled sanitary pad that she hasn't taken out since she inserted it the previous day.

EXT. BUS STOP - DAY

Bertha appears from the public toilet. She hovers nervously on the outskirts, as people begin to board.

Soon it is only her and the strange Man left. The BUS DRIVER closes the luggage compartment and Bertha realises she's about to miss her chance.

BERTHA

Does this bus stop in Jamieson?

The strange man's ears prick up.

BUS DRIVER

What you wanna go to Jamieson for?

Bertha is taken aback. The bus driver smiles before realising Bertha doesn't get his humour.

BUS DRIVER

Just kidding, love. We stop at all

sorts of shit holes.

Bertha looks hopeful again, and pulls out the jar of coins from her bag.

BERTHA

One ticket please?

The bus driver eyes her dubiously.

BUS DRIVER

How old are you? 


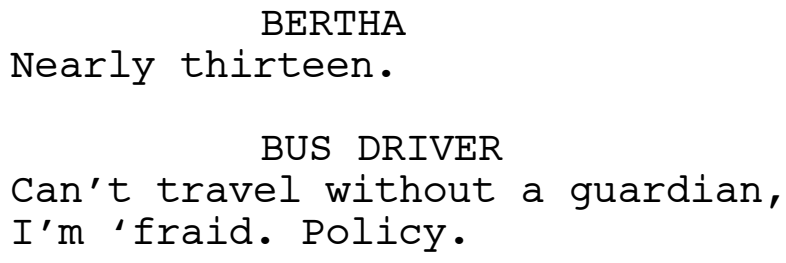

Bertha looks heartbroken.

Bertha racks her brain for an excuse when the Strange Man steps in. He has an Australian accent.

$$
\begin{aligned}
& \text { STRANGE MAN } \\
& \text { I'm her guardian. }
\end{aligned}
$$

The Strange Man puts his hand on Bertha's shoulder. Bertha freezes with fear.

$$
\begin{gathered}
\text { STRANGER MAN } \\
\text { I'm travelling with her. }
\end{gathered}
$$

The bus driver clearly doesn't believe it, but doesn't care enough to argue.

$$
\text { BUS DRIVER }
$$

Bertha slips from under the man's grasp and counts out fifteen dollars of coins from the jar. The bus driver is not pleased, and the Strange Man gives a sidelong glance at Bertha's coin jar.

$$
\begin{gathered}
\text { STRANGE MAN } \\
\text { She's been saving up for yonks. }
\end{gathered}
$$

When she's done counting out all the money, she heads onto the bus.

INT. BUS - DAY

Bertha takes a seat by a window. She makes herself as small as possible.

The Strange Man hops on board and takes a seat across the aisle from her.

Bertha tries to avoid eye contact.

$$
\text { STRANGE MAN }
$$

No need to thank me.

Bertha glances at him.

$$
\text { Thank you. }
$$$$
\text { BERTHA }
$$

The Strange Man shrugs it off. 
Happy to help a little lady reach

her destiny.

The Strange Man laughs at himself.

STRANGE MAN

I mean destination.

The stranger winks at her, then nestles into his seat keeping his eye on Bertha all the while.

The bus's engine starts up, and they move off. Bertha stares out the window with a mix of terror and excitement in her eyes.

INT. JESSOP HOUSE, LOUNGE - NIGHT

Brett, Kyle, Jake and Callum are all sprawled out in the lounge watching TV, just like the night before.

Trent enters from the kitchen with a dish filled with microwaved sausages, a pottle of tomato sauce and a packet of white bread. He plonks them on the coffee table.

The boys each grab a sausage and a bit of bread, and take turns at pouring sauce over them before shovelling them into their mouths.

TRENT

Bertha! Dinner!

Kyle shakes his head.

KYLE

Don't think she's home.

Trent looks confused.

\section{Where is she?}

\section{TRENT}

Brett shrugs, uncaring. Trent has a thought that answers his question.

\section{TRENT}

She say something about a camp yesterday?

The boys all shrug, not really listening.

Trent takes a seat on the floor, grabs a sausage from the dish, and munches into it, unconcerned. 
EXT. COASTLINE - NIGHT

Through heavy rain, the bus zooms down a bumpy road running adjacent to a severely eroded cliff that overlooks the rough ocean.

The coastline is embedded with bits of old crumbled road, houses, and fence posts.

INT. BUS/COASTLINE - NIGHT

Bertha is asleep - her head resting on an uncomfortable angle against the window.

One of the back windows in the bus is jammed open, causing rain water to leak into the now near-empty bus.

Water trickles down the aisle. When it reaches the bus driver's feet, he slams on the brake.

\section{Bloody Jamieson.}

BUS DRIVER

Bertha wakes up as the bus lurches. She gawks out the window at the shambolic coastline, and notices a stream of smoke wafting up from behind some large rocks.

The bus continues on, and the smoke fades in the distance.

EXT. JAMIESON BUS STOP - NIGHT

The bus pulls into the small town-centre of Jamieson.

INT. BUS - NIGHT

Bertha nervously gathers her things. The Strange Man does the same from across the aisle.

\section{STRANGE MAN}

Your family know you're here?

Bertha overtly avoids eye contact with the Strange Man.

$$
\text { Yes. }
$$$$
\text { BERTHA }
$$

The Strange Man sees through her lie.

STRANGE MAN

I could escort you to wherever it

is you're going, if you like?

Bertha's voice squeaks with terror. 


$$
\text { I'm fine, thank you. }
$$

Bertha bolts down the aisle, and jumps off the bus.

The Strange Man takes a moment, looking guilty for making Bertha feel scared. He slowly makes his way towards the front of the bus.

EXT. JAMIESON BUS STOP - NIGHT

Across the road from the bus stop is the dairy with the blackboard out in front of it.

ROY (24) - the dairy cashier - bends over the blackboard, wipes the "2" off of the "2 sleeps to go!" and replaces it with a "1". He forgets to get rid of the "s" in "sleeps", so it now reads: "1 sleeps to go!"

Roy heads back into the dairy.

Bertha, now drenched by the rain, runs across the road to the dairy.

The bus pulls away, and the Strange Man stands on the footpath, watching Bertha go into the dairy.

INT. DAIRY, JAMIESON - NIGHT

The doorbell jingles as Bertha enters.

The shop is cluttered with dusty, overpriced items.

Roy stands behind the counter watching Bertha closely - he chews bubblegum intensely.

Bertha grabs a packet of chips, and takes it to the counter.

Roy rings it up on the register, and speaks to Bertha much louder than he needs to.

$$
\begin{aligned}
& \text { ROY } \\
& \text { BUCK FIFTY. }
\end{aligned}
$$

Bertha hands over the coins, and Roy puts them into the register.

Bertha lingers, wanting to ask him a question. Roy blows a large pink bubble out of his gum. It pops and deflates pathetically. He starts chewing again.

ROY

$$
\begin{aligned}
& \text { I'VE NOT SEEN YOU IN HERE BEFORE, } \\
& \text { HAVE I? }
\end{aligned}
$$

Bertha shakes her head. 


\title{
ROY \\ YOU HERE FOR JAMIESON'S PREDICTION?
}

Bertha doesn't understand. She shakes her head. Roy looks disappointed.

\author{
ROY \\ WE WERE HOPING IT WOULD BRING IN \\ SOME TOURISTS.
}

Bertha finally speaks up, but she's quiet and nervous.

\section{BERTHA}

Do you know where 20 seed Road is?

Roy squints at her. He points to a sign on the counter:

“Hi, I'm Roy and I'm hard of hearing. Please enunciate clearly."

Roy forms shapes with his lips, to demonstrate.

Bertha, feeling awkward, repeats herself, but makes sure she enunciates.

\section{BERTHA \\ DO YOU KNOW WHERE 20 SEED ROAD IS?}

Roy extracts the bubblegum from his mouth and slaps it underneath the counter top. He eyes Bertha, curiously.

\section{THIS A JOKE?}

ROY

Bertha shakes her head, earnestly.

Roy disappears into a back room, the returns with a framed front cover of an old newspaper. He looks down at it.

\section{ROY}

BIT WEIRD THEY FRAMED IT, BUT I

GUESS IT'S THE ONLY TIME WE'VE BEEN

IN THE NATIONAL NEWS.

He hands it over to Bertha.

Bertha looks at it - the headline reads:

"LANDSLIDE KILLS FOUR IN SUNNY CLIFF: Four people are dead after severe weather caused a cliff to collapse in the coastal town."

The article provides a map of the town, and pinpoints where the slip occurred: Seed Road, a residential road that once existed on the now-eroded cliff that Bertha observed from the bus. 
ROY
THERE'S NOT BEEN A SEED ROAD SINCE.

Bertha looks at the date on the newspaper - July 24, 1967.

NOT IN 1986?

BERTHA

Roy shakes his head.

Bertha pulls out the birth certificate and glances at it.

$$
\text { BERTHA }
$$

Do you know an Odette Wilson?

Roy squints at her since she hasn't enunciates clearly.

$$
\text { WILSON? ROY }
$$

Bertha nods, hopeful. Roy shakes his head.

Bertha is completely devastated - her mistake washing over her.

Her lip trembles, and Roy looks unequipped to deal with it.

Bertha is about to head for the exit when she sees the strange man standing outside.

She panics and turns back to Roy

$$
\text { BERTHA }
$$

DO YOU HAVE A PHONE I CAN USE?

Roy hesitates, but pulls out a landline from under the counter.

ROY

BE QUICK COS THE FESTIVITIES HAVE

ALREADY STARTED.

Bertha leans over, picks up the receiver and punches in a number, her fingers trembling.

Roy disappears into the back room.

INT. JESSOP HOUSE, LOUNGE - NIGHT

Trent is coma'd out on the floor again, and Bertha's four brothers are still lounging around, watching TV. The phone in the kitchen rings.

Trent sleeps through it, but the brothers all hear it and, in impressively quick succession, yell: 


\section{Bags not! \\ KYLE / BRETT/ CALLUM}

Bags--

JAKE

--Kyle, Brett and Callum all laugh and point at Jake.

Jake pulls himself up, reluctantly.

INT. JESSOP HOUSE, KITCHEN - NIGHT

Jake picks up the phone.

$$
\begin{array}{ll}
\text { Hello? } & \text { JAKE } \\
\text { Jake? } & \text { BERTHA (PHONE) } \\
\text { Bertha? } & \text { JAKE } \\
& \\
\text { Can you come get me? }
\end{array}
$$

Jake frowns, confused.

$$
\text { Where are you? }
$$

Bertha pauses, then speaks guiltily.

Jamieson.

$$
\text { BERTHA (PHONE) }
$$

Jake looks vacant.

$$
\text { Where? JAKE (INTO PHONE) }
$$

INT. JAMIESON DAIRY - NIGHT

Bertha looks confused now.

\section{BERTHA}

Jamieson. Where the birth certificate said my birth mum is from. Except they musta got it wrong or something cos the address isn't an address anymore. So I really need you to come get me. 
INT. JESSOP HOUSE, KITCHEN - NIGHT

Jake looks completely baffled.

JAKE

What are you talking about? We made

all that stuff up.

INT. JAMIESON DAIRY - NIGHT

Bertha has fallen silent.

JAKE (PHONE)

There's a real place called

Jamieson?

The rain starts to pelt down again outside. Tears stream down Bertha's face.

$$
\text { BERTHA }
$$$$
\text { JAKE (PHONE) }
$$

Jesus Christ. How did you...? How?!

Bertha's breath quickens, humiliated and panicked.

$$
\text { BERTHA }
$$

Please come get me.

$$
\text { JAKE (PHONE) }
$$

Where are you calling from?

Bertha sees that the Strange Man is walking away into the darkness.

In the dairy.

$$
\text { BERTHA }
$$

\section{JAKE (PHONE)}

OK. Stay put - we'll get there as

soon as we can.

Bertha relaxes ever so slightly.

$$
\text { BERTHA }
$$

Thank you.

Bertha hangs up the phone.

Bertha goes over to the door, and is watching the rain pour down outside, when Roy reappears from the back room.

$$
\begin{gathered}
\text { ROY } \\
\text { CAN YOU PLEASE LEAVE? }
\end{gathered}
$$


Bertha turns to him, and Roy flinches when he sees her blotchy, tear-drenched face.

\section{BERTHA}

Can I please stay in here until my

brothers come get me?

Roy squints, having not heard her.

Bertha realises her mistake and opens her mouth to speak louder but Roy - clearly not wanting to hear her interrupts.

\section{ROY \\ FESTIVITIES!}

Roy gestures outside where, across the road, a park area is crammed with tents around a bonfire.

Bertha's posture shrinks as Roy pushes past and he opens to the door for her to leave through.

Bertha trudges out into the rain. Roy flicks the dairy light off, steps outside, then locks the door.

Bertha stands under the awning of the dairy, and watches Roy run across the road to where the tents and marquee are.

Bertha takes a seat on the concrete, and hugs her backpack to keep warm.

INT. JESSOP HOUSE, BEDROOM - NIGHT

All four brothers are crammed in the bedroom, having a heated, but hushed, conversation.

$$
\begin{gathered}
\text { KYLE } \\
\text { (to Jake and Callum) } \\
\text { You two are such dickheads. }
\end{gathered}
$$

Jake is holding a NZ road atlas in his hand.

$$
\text { JAKE }
$$

According to this it should only

take a couple of hours to get

there.

Jake checks to see that everyone is on board. Kyle laughs. KYLE

I'm not going with you.

JAKE

You can stay behind and cover us if

Dad wakes up, then. Brett, can you

drive? 
Brett shakes his head, annoyed but relenting •

Whatever.

BRETT

CALLUM

I can't believe how retarded she

is. Like, she's seriously special

needs.

JAKE

Shut up, Callum. This is serious.

Callum pulls a face at Jake.

Jake shakes his head at him, before leaving the room. Callum and Brett follow after him.

EXT. COUNTRY ROAD - NIGHT

A white van is parked up on the side of a country road. Its headlights are on, and are pointing at a sign post that marks the turn off to "Jamieson".

The Strange Man is standing on a ladder leaning against the signpost, and is unscrewing the sign from it.

He carries the sign down the ladder, and places it, and the ladder, in the back of his van which is jam packed full of shrubs and leafy tree branches.

The Strange Man proceeds to heave the shrubs and branches out of the van, and places them at the mouth of the turn-off.

EXT. JAMIESON DAIRY - NIGHT

Bertha remains scrunched up in her tight ball, watching the flickering flame from the bonfire, when she hears a sing-along start up.

Curious, Bertha pulls herself up and heads over to the park.

EXT. PARK, JAMIESON - NIGHT

Bertha stands on the outskirts of the park, hidden in the shadows of a bunch of trees, and watches the TOWNSPEOPLE (excited children, and drunk adults) stand around the bonfire singing a song.

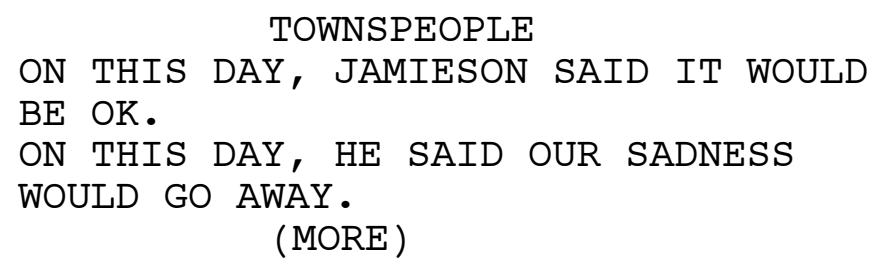




\section{TOWNSPEOPLE (CONT ' D) \\ ON THIS DAY, HE SAID WE'LL BE \\ SINGING "HOORAA-AAY"!}

The townspeople cheer and laugh together when they come to the end of the song.

They all break off into separate groups. A WOMAN takes a seat on a bench near where Bertha is standing, and is followed by a priest - FATHER DAVID (70s) - who takes a seat next to her.

$$
\begin{aligned}
& \text { FATHER DAVID } \\
& \text { Pity about the rain. }
\end{aligned}
$$

The woman nods. They sit in silence for a moment while Father David surveys the crowd of people.

FATHER DAVID

Any sign of Odette?

Bertha's eyes go wide on hearing this name.

The woman sighs.

\section{WOMAN}

Don't know why you bother with that

lady.

FATHER DAVID

She's as much a part of this town

as anyone else.

WOMAN

She belongs in the loony bin.

Father David tries to ignore this comment. He shakes his head.

FATHER DAVID

When I spoke to her the other day

she seemed so enthusiastic about

all this. Asking all sorts of questions.

The woman glares at Father David.

FATHER DAVID

I think I'll go and check on her.

Father David stands up.

\section{WOMAN}

No one here will be thanking you.

Father David shakes his head again.

FATHER DAVID

No one ever thanks me. 
Father David heads through the trees, and Bertha has to duck down so as not to be seen.

Once Father David is a certain distance away, Bertha follows after him.

EXT. COASTLINE - NIGHT

Bertha watches Father David clamber over the debris-ridden coastline towards the stream of smoke rising from behind a cluster of boulders and bush.

Bertha continues to follow Father David at a distance, until a make-shift house comes into view.

The house is built on the foundation of an old house clearly uplifted from the ground long ago. The house itself is made up of bits of other houses, and leans on a slightly wayward angle.

EXT. ODETTE'S HOUSE - NIGHT

Bertha ungracefully makes her way over the rocks to the side of the house, and listens as Father David knocks on the door around the corner from her.

Bertha peers round the corner and sees that there is a knitted, life-size doll dangling by a rope through a window and down the side of the house.

Father David continues to knock.

FATHER DAVID

Odette! I know you're in there.

Father David knocks again, but still gets no answer.

FATHER DAVID

We're all down at the park if you

want to join us.

Still no response.

FATHER DAVID

OK, then. You'll be missed.

Father David lowers his head, and heads back towards the campsite. Bertha ducks around the other side of the house to remain out of sight.

When Father David's far enough away, Bertha creeps around the side of the house to the front door.

Bertha, astonished, stands in front of the door - which is clearly from a different house - but has an old inscription on it that says: "20 seed Road". 
In the corner of her eye, Bertha sees the knitted doll is being pulled back into the house through the window.

Bertha hesitantly makes her way over to the window - a hole in the side of the house. ODETTE CHEN (50s) is hanging a blanket over the hole when she sees Bertha standing there.

Odette drops the blanket, and stares at Bertha, stunned.

\section{Bertha?}

ODETTE

Bertha doesn't know what to do with herself. Eventually Odette opens the door and stands in the doorway gawking at Bertha.

BERTHA

What's going on?

Odette eventually snaps out of it and ushers Bertha inside.

EXT. COUNTRY ROAD - NIGHT

A huckery car hoons down the country road.

INT. CAR - NIGHT

Brett is driving the huckery car.

Jake, anxious, is sitting in the front seat, intermittently glancing down at the atlas in his lap.

Callum is in the back seat, snoozing.

They drive silently, through the dark country road.

JAKE

Should be a right turn coming up onto Reel street.

Jake and Brett look to their right, looking out for the turnoff. But it never comes, so they keep driving on - past the concealed turn off.

INT. ODETTE'S HOUSE - NIGHT

The inside of the house is surprisingly homely for what it is.

Most of the furnishings are set up at the end of the house furthest away from the crashing waves.

A pit of coals burns in a make-shift kitchen - a hole in the ceiling releases the smoke, but it also lets in rain that creates more smoke. 
Odette is boiling a pot of tea over the coals, and shoots Bertha perplexed glances every now and again.

Bertha nervously looks around - there are huge chunks of grassy soil scattered around the place, as well as big boulders carved into odd shapes.

Bertha sees a rock-table displaying three framed pictures. She inspects them. First is a portrait of a middle-aged Chinese couple from the 60s. Next to it is a framed postcard of an idyllic-looking 'Sunny Cliff' - clearly before the landslide.

And then Bertha picks up a photograph that nearly causes her to faint.

This photo is yellowed and faded with age, but there, sitting on the front steps of an old farm-house in the mid 1960s, is a girl that looks exactly like Bertha.

Bertha staggers over to a mouldy arm chair and takes a seat. She stares at the picture, barely blinking.

Is this me?

BERTHA

Odette brings Bertha a cup of tea, and takes a seat opposite her on a wooden park bench.

ODETTE

Is it?

(genuine question)

Bertha doesn't know what this means. She pulls out the birth certificate, and hands it to Odette.

\section{BERTHA}

My brothers made this as a prank to make me think I was adopted.

Odette frowns as she reads over it.

\section{A prank?}

ODETTE

Bertha nods, spaced out.

Odette inspects the birth certificate closely. She smears some of the now-blotchy ink with her thumb.

\section{ODETTE \\ How did they know my birthday?}

Bertha is so overwhelmed that she looks like she's going to cry again.

BERTHA

That's your actual birthday? 
Odette nods, spaced out.

ODETTE

And 20 Seed Road. That was Bertha

Manning's address.

BERTHA

Bertha Manning?

Odette nods to the photograph in her hands.

ODETTE

She was my childhood friend before

she died in the landslide.

Bertha looks at the photo of her lookalike again.

BERTHA

What's happening?

Odette takes a moment to gather herself and her thoughts, when she springs out of her seat, having come to some sort of realisation.

ODETTE

Fate has obviously sent you here to help me.

BERTHA

Help you?

Yes!

ODETTE

Odette claps her hands, excited and grateful.

ODETTE

You might have heard that the town

is expecting a miracle tomorrow.

Something that's meant to restore

the town to its former glory.

There is a hint of frustration as Odette continues.

ODETTE

All because Jamieson - an old white

man with a degree - predicted it

fifty years ago after the landslide

happened. You know the town wasn't

always called Jamieson, right? They

went and renamed it after him.

Odette shakes her head to herself, then looks Bertha dead in the eyes. She hesitates before saying the next bit.

ODETTE

I made the whole thing up, Bertha. 
Odette has clearly never said this out loud before, and she's overwhelmed with relief.

Bertha stares blankly at her - completely lost.

ODETTE

I didn't think it would catch on

like it did. I mean, jeepers, I

just wanted to give the people a

bit of hope after the landslide.

Something to get them through the

hard times... I thought they'd

forgotten about it, but...

Odette shakes her head, exasperated.

\section{ODETTE}

The kids are even being taught it

at school. They sing songs about

the man!

Odette retrieves the knitted doll that's now on the floor under the make-shift window.

ODETTE

So I've been toiling away, trying

to come up with ways to give them

something.

She wags the doll, and gestures to the boulders and chunks of grassy soil.

ODETTE

But now you've been sent to me.

Odette goes over to Bertha, and puts her hands gently against Bertha's face.

$$
\begin{gathered}
\text { ODETTE } \\
\text { You're the miracle. }
\end{gathered}
$$

Bertha is completely flummoxed.

INT. CAR - NIGHT

The Jessop brothers are now driving in the other direction, still looking for Reel street. Callum is now awake, and is also keeping his eyes peeled.

Brett is gripping the steering wheel.

JAKE

It should definitely be around here

somewhere.

Callum turns and looks through the back window. 


$$
\begin{gathered}
\text { CALLUM } \\
\text { Maybe it is further that way. }
\end{gathered}
$$

Brett swerves to the side of the road, and stops the car with a skid. Jake and Callum are thrown around in their seats.

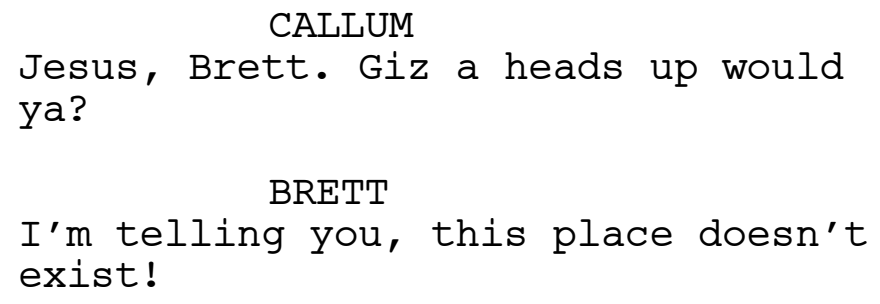

Brett looks to the others - he's being dead serious, but the others don't believe him.

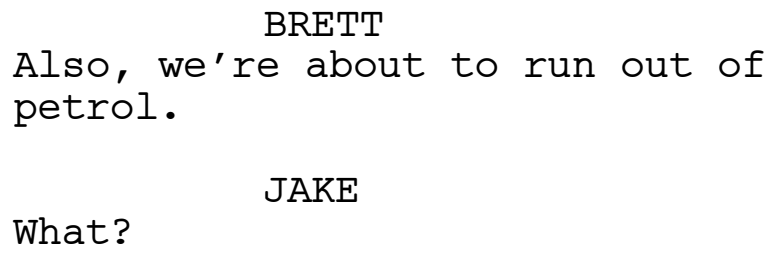


ODETTE

You won't have to do much - just

make an appearance as Bertha

Manning's ghost, and maybe give

some reassuring words from beyond

the grave, then disappear again.

That's all. Easy as pie. They'll

love it.

Odette tries to hand the dress over, but Bertha shakes her head, and pulls herself up.

\section{BERTHA}

My brothers will be here soon.

Bertha makes a move for the door, but odette grabs onto her shoulder to stop her.

$$
\text { You can't leave now! }
$$

Bertha is panic-stricken.

\section{BERTHA}

You're scaring me.

Bertha struggles out of Odette's grasp, and lunges for the door.

Odette launches after her.

$$
\text { I need you! }
$$

Bertha scurries through the door.

EXT. ODETTE'S HOUSE - NIGHT

Bertha tumbles over the rocks towards the road.

$$
\text { ODETTE }
$$

The town needs you!

Odette chases after Bertha.

$$
\text { ODETTE }
$$

You mustn't defy fate!

EXT. COUNTRY ROAD - NIGHT

The huckery car slowly drives down the country road through the rain when the engine starts making a spluttering sound. 
INT. CAR - NIGHT

Brett grips the steering wheel as the engine ticks and splutters until it is completely kaput.

Thar she blows.

CALLUM

BRETT

(irate)

That's not what that means.

Brett steers the car to the side of the road, and turns off the ignition.

JAKE

What should we do?

Brett looks out the window at the pouring rain.

$$
\text { We're gonna have to walk. }
$$

Callum is pissed off.

$$
\text { I'm gonna murder her } \text {. }
$$

The brothers all clamber out of the car, Jake grips the road atlas tightly.

EXT. DAIRY - NIGHT

Bertha, huffing and puffing, jogs back to the dairy - making sure to avoid the campsite festivities.

Across the road, Odette appears - also exhausted. She watches Bertha take a seat on the ground, and huddle into an inconspicuous ball.

Odette is about to cross the road to approach her, when she sees the tents in the park, and has an idea.

She heads to the campsite.

EXT. PARK, JAMIESON - NIGHT

Odette creeps up to the park area where everyone has

retreated into their tents to stay out of the rain.

Odette stands up on a picnic table, and yells as loud as she can: 
There is a little bit of movement from some of the tents, but all is silent.

Odette stomps her feet on the table.

$$
\text { ODETTE }
$$

Wake up! The prediction is

happening now!

There is a murmur of confusion. A couple of people turn their torches on in their tents.

$$
\text { Get ready, people!! }
$$

The sound of tents being unzipped makes Odette smile, excited.

Several sleepy and confused townspeople emerge from their tents - including Roy and Father David.

A man, NICK (30s), who holds his grizzly FOUR-YEAR-OLD in his arms, speaks up - furious.

$$
\begin{gathered}
\text { NICK } \\
\text { You think this is funny? }
\end{gathered}
$$

They all stare at Odette who continues to stand on the picnic table - her excitement has turned into dismay.

$$
\text { ODETTE }
$$

No...The prediction, that you've

all been counting down for, is

happening...

Some people laugh. Some are confused. Others are concerned for Odette's wellbeing. But most are furious.

$$
\begin{aligned}
& \text { FATHER DAVID } \\
& \text { (concerned) } \\
& \text { Odette - you know the prediction } \\
& \text { isn't actually real, right? }
\end{aligned}
$$

Odette sways dizzily with this question.

$$
\text { ODETTE }
$$

What do you mean? Of course it's real.

Some people go back into their tents, realising there is no emergency •

$$
\text { It's a myth. }
$$

Odette deflates even more, struggling to comprehend. 
ODETTE

What's with all this, then?

Odette gestures to all the tents.

WOMAN

You know Santa isn't real, right?

Some people snigger. More people disappear back into their tents, muttering.

The daughter has heard the comment about Santa, and looks up at Nick, as dismayed as Odette is. Nick tries to comfort her, but she starts to cry.

ODETTE

But you named the town Jamieson?

Father David looks sorry for Odette.

FATHER DAVID

That was more of a joke that took

hold.

Odette, dumbfounded, looks at Father David, and then at all the people still staring at her like she's a freak show.

FATHER DAVID

I, for one, was against it.

Father David steps over to her and holds out his hand to help her down from the table.

FATHER DAVID

This is why you should try to be

more involved in the community,

Odette.

Odette doesn't take his hand.

Odette watches on as the rest of the people disappear back into their tents.

FATHER DAVID

What did you think was happening?

Huh?

ODETTE

FATHER DAVID

The prediction?

Odette is silent for a moment. She finally gets down from the table.

Nothing. Just... 
Odette realises how silly her plan was.

ODETTE

Bertha Manning had returned from

the dead.

Father David looks disturbed.

\section{Bertha Manning?}

FATHER DAVID

Odette trudges away from the campsite and back towards her house.

Father David follows after her.

FATHER DAVID

How were you going to pull that off?

EXT. COUNTRY ROAD - NIGHT

The three brothers trudge down the road in the pelting rain when Jake spots the concealed road to Jamieson.

Jake sprints over to it, and turns to his brothers.

Look!

JAKE

Jake discards the branches, and the brothers make their way down the dark road.

EXT. ODETTE'S HOUSE - NIGHT

Father David is still shadowing Odette as she heads back towards her house.

Odette stops walking, annoyed, and turns to Father David.

ODETTE

I'd like to make it very clear -

I'm the one who started the rumour

about the prediction.

Father David looks confused.

ODETTE

I'm the one who made Jamieson up.

This is clearly news to Father David, but it's not what he's interested in. 
FATHER DAVID

OK, but how were you going to prove

Bertha Manning had returned from

the dead? And why her?

Odette frowns.

\section{ODETTE}

Why do you care?

Father David catches himself, and tones down his intensity.

\section{FATHER DAVID}

I'm just curious. It's curious.

Odette takes a moment to consider whether or not to tell him.

ODETTE

A girl who, I swear, was the

spitting image of Bertha Manning

showed up at my house earlier.

Odette shakes her head, still disbelieving.

ODETTE

She claimed her name was Bertha, and she had all this information

about me.

Father David frowns as he takes all this on board.

ODETTE

Someone must have put her up to it.

FATHER DAVID

Where is the girl now?

Odette eyes him suspiciously now.

$$
\begin{gathered}
\text { ODETTE } \\
\text { I'm not telling you. }
\end{gathered}
$$

Odette disappears into her house.

Father David scours Odette's house, fruitlessly searching for Bertha Manning's lookalike, before giving up and heading back towards the road.

Odette can be seen poking her head out her window, watching Father David leaving.

She soon appears at her door, and follows after Father David. 
EXT. DAIRY, JAMIESON - NIGHT

Bertha, still sitting on the concrete, is staring at the picture of Bertha Manning that she swiped from odette's house.

The rain starts to pour down again, when the strange man pulls up in his van.

He opens his door, and when Bertha sees who it is, she shuffles backwards in fear.

$$
\begin{aligned}
& \text { STRANGE MAN } \\
& \text { I'm not going to hurt you, Bertha. }
\end{aligned}
$$

Bertha's eyes go wide.

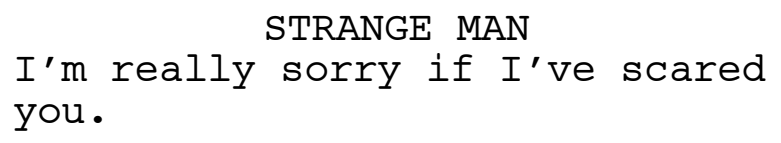

The strange man sees in Bertha's terrified expression that this isn't going well.

$$
\begin{aligned}
& \text { STRANGE MAN } \\
& \text { My name's Howard. }
\end{aligned}
$$

Howard flashes Bertha a friendly smile.

\section{BERTHA}

How do you know my name?

Howard leans over and retrieves a folder from his glove compartment.

\section{HOWARD}

You're a big piece of a puzzle that

I've been trying to solve for most

of my life.

Bertha looks perplexed.

Howard sees that she's holding a picture of Bertha Manning. HOWARD

I see you already know about Bertha

Manning.

Howard hops out of the van, and hands over the folder to Bertha.

\section{HOWARD}

Identical Berthas throughout history.

Bertha opens it up and sees a series of photographs of various women, and one man, who are all of different ages and from different eras but look very similar to one another. 
It's like looking at an age progression of Bertha throughout her entire life span.

Bertha turns the photographs over - each have a name, year, and age:

Bertha Anne, age 74 (1937)

Berta Lorenz, age 42 (1939)

Bertie Nobody, age 26 (1943)

Bertha Greive, age 18 (1951)

Bertha Manning, age 14 (1967)

Bertha Jessop, age 12 (1999)

BERTHA

I don't understand.

Howard laughs.

Nor do I. Yet.

Bertha shakes her head, baffled.

\section{HOWARD}

What I'm sure of is that you're

part of something miraculous, Bertha.

Bertha looks down at the photographs again.

HOWARD

And if you come with me now, we can

figure this out together and maybe

find an answer to the mysteries of

the universe itself!

Bertha doesn't move, other than to look Howard up and down.

BERTHA

But my brothers are coming .

Howard shakes his head.

HOWARD

They know about all this, and they

want you to come with me.

Bertha frowns.

BERTHA

You talked to them?

Howard nods. 
BERTHA

They said they don't want me to

come home?

Howard hesitates, and then shakes his head again. Bertha looks heart-broken.

HOWARD

I hate to interfere and force

things, so if you don't want to

come with me then that's fine. I'll

have to accept that it wasn't meant

to be.

Bertha takes a moment to consider.

BERTHA

This isn't another prank?

HOWARD

Would have to be a pretty elaborate prank.

Bertha flicks through the photographs again.

BERTHA

I want to come.

Howard smiles, immensely relieved, and opens the passenger seat door for her.

Bertha hops in.

Howard gets into the driver's seat, and drives the van into the distant depths of Jamieson.

At that, Father David tumbles onto the road from the rocky shore, and heads back towards the campsite when there is a loud crunching sound followed by a wallop that booms through the air.

Father David heads towards where the sound came from - the road leading into Jamieson.

EXT. COUNTRY ROAD - NIGHT

The brothers continue to trudge down the road through the rain when they hear the same crunching and walloping sound.

The brothers run to see what it is, and find themselves on the outskirts of a large landslide that completely blocks the road into Jamieson.

The brothers back away to safety, as more soil and gravel tumbles down the hill.

The brothers all look at each other, stunned. 
EXT. JAMIESON, ENTRANCE - NIGHT

Odette has appeared, and stands by Father David, on the other side of the landslide. She looks completely rattled.

Other townspeople are emerging from the tents to see what's happening •

ODETTE

This is how it happened last time.

EXT. JAMIESON, WINDY STREET - NIGHT

Howard drives up a hill on a windy road that is surrounded by pine trees.

Bertha is surveying the photographs more closely, when they both hear the sound of the landslide.

What was that?

BERTHA

Howard continues driving, unperturbed.

HOWARD

If my calculations are correct, it's a landslide.

Bertha looks confused.

Howard turns off down a gravel driveway that leads into a thicket of pine trees.

EXT. HOWARD'S HOUSE - NIGHT

Howard parks his van outside a homely-looking country house that's clearly been well looked after and loved.

Howard gets out and opens Bertha's door for her.

Come on in.

Bertha tentatively gets out of the van.

INT. HOWARD'S HOUSE - NIGHT

Howard opens the front door for Bertha. She steps inside.

She looks around in awe at Howard's cozy home. Everything inside is clean, warm and inviting.

And there's a proper dining table, with a table-cloth and everything. 
Woah.

Bertha gawks at the fireplace that is roaring in the lounge, in front of a comfy-looking sofa.

\section{BERTHA}

I like your house.

Howard smiles.

$$
\text { I'm glad. HOWARD }
$$

Howard ushers Bertha to take a seat at the dining table. HOWARD

I'll just get my wife.

Bertha is surprised and relieved to hear Howard has a wife. Howard disappears down a hallway.

$$
\text { Darling? }
$$

$$
\text { HOWARD (O.S.) }
$$

Bertha already feeling at home, creeps into the kitchen and quietly opens the fridge.

Bertha's eyes go wide as she sees how packed with deliciouslooking food it is.

EXT. JAMIESON, ENTRANCE - NIGHT

Most of the townspeople are now standing on the outskirts of the landslide. They all look spooked.

The cliff is basically completely flattened, as if there was never a cliff to begin with.

\section{WOMAN}

That land might be usable again.

$$
\text { NICK }
$$

This is a coincidence, right?

FATHER DAVID

Or something divine.

$$
\text { WOMAN }
$$

Or Jamieson was real.

Odette fidgets at this comment. She turns to Father David and whispers to him. 
Odette scurries away, panicked, before Father David can respond.

In the sky a helicopter approaches.

INT. HOWARD'S HOUSE - NIGHT

Bertha is still standing in front of the open fridge.

Howard enters with his WIFE (64) who is overwhelmed at seeing Bertha.

HOWARD

Bertha.

Bertha jumps in fright, and quickly closes the fridge, embarrassed.

BERTHA

Sorry

Howard smiles.

HOWARD

Bertha Jessop, I'd like you to meet

Bertha Manning.

Bertha looks at BERTHA MANNING, surprised.

Bertha Manning stares back at her, with tears in her eyes.

BERTHA MANNING

She looks just like I did, Howard.

End of Episode. 

THE CHANCES

Episode Two:

"Bertha Manning"

Written by

Alison McLachlan 

EXT. OCEAN (1967) - DAY

MICHAEL (25 - wearing a formal, cruise-ship uniform) is paddling a row boat in the middle of the ocean, panicked.

A large cruise ship can be seen behind him in the distance.

EXT. SUNNY CLIFF, CHURCH (1967) - DAY

The ground is dotted with large puddles of rain.

Dark clouds hang over the church as the CONGREGATION mill about outside after a service.

JUNE MANNING (50s) dominates a conversation with FATHER DAVID (40s) who looks like he wants to escape.

EXT. CHURCH GRAVE YARD - DAY

BERTHA MANNING (14 - a slightly older-looking version of Bertha Jessop, and wearing the 1960s frock that Odette tried to get Bertha Jessop to wear in the previous episode) is sitting against the side wall of the church, which looks out to a graveyard in the distance.

Bertha Manning is clutching a stick and rapidly rubbing it back and forth against her thigh. A red mark is emerging, but she suppresses her pain.

ODETTE CHEN (16) approaches Bertha Manning with curiosity.

ODETTE

What are you doing?

Bertha Manning looks up at Odette like she's the weird one.

BERTHA MANNING

Find a stick and I'll show you.

Odette searches the ground, and returns with a stick.

BERTHA MANNING

Rub it against your skin like this.

Bertha Manning rubs the stick against her thigh.

Odette looks confused, but takes a seat next to Bertha

Manning, pulls her skirt up and proceeds to rub the stick against her thigh.

Odette grimaces at the pain, and rubs it a little more softly.

Harder!

$$
\text { BERTHA MANNING }
$$


Odette hesitates, then rubs it harder - her skin goes red, and starts to bleed. Odette stops.

Bertha Manning looks smug.

BERTHA MANNING

You're not special like me.

Bertha Manning continues to rub the stick against her thigh not nearly as hard as Odette was.

BERTHA MANNING

I don't feel pain like everyone

else.

Odette looks disappointedly at her grazed thigh.

ODETTE

It didn't hurt me that much.

Bertha Manning rolls her eyes.

BERTHA MANNING

When I'm older and I go to the

doctor for a check up, I'll get on

the news cos they'll have found out

I've been living with tuberculosis

my whole life without realising.

Odette clearly doesn't believe this, but pretends to be impressed anyway.

\section{BERTHA MANNING \\ People die from tuberculosis.}

Bertha Manning swoons when she spots Father David speaking with June in the distance. Bertha Manning tosses away her stick.

BERTHA MANNING

And when Father David finds out, he'll be so proud that I'm his wife.

Odette grimaces and laughs.

ODETTE

You want to marry Father David?

Bertha Manning looks furious.

So what?

BERTHA MANNING

Odette stops smiling.

ODETTE

Father David is old and disgusting. 
Bertha Manning shakes her head and folds her arms, superior.

BERTHA MANNING

You're too immature to appreciate a good man when you see one.

I'm older than you.

Bertha Manning ignores Odette.

BERTHA MANNING

He already has a crush on me, so

now I just need to get him to fall

in love with me.

ODETTE

But Father David's married to God.

Bertha Manning pulls a face.

BERTHA MANNING

I'm better than God.

EXT. SUNNY CLIFF, CHURCH - DAY

Father David is still stuck in the conversation with June.

JUNE

Truly, my Bertha excels at

everything she tries.

Father David subtly rolls his eyes.

FATHER DAVID

I'm quite sure she's the apple of

your eye, Mrs. Manning, but - as

I've said before - you must be

careful not to spoil the child.

June laughs, defensive. Father David continues.

FATHER DAVID

I've observed some unfavourable

behaviour from her in Sunday

School. She's competitive,

arrogant, and - quite frankly -

unladylike.

June can't believe what she's hearing, but then she gives a look of understanding.

JUNE

Well, it's understandable for her

to get frustrated since the other

children aren't as advanced as her. 
Father David rolls his eyes to himself.

He sees MR. \& MRS. CHEN in the distance, trying to catch his eye.

FATHER DAVID

Excuse me, Mrs. Manning.

Father David makes his exit, but ignores the Chens and greets a FAMILY instead.

June looks around, unsure what to do with herself now, when she is horrified to see Bertha Manning and Odette sitting together down the side of the church with their skirts pulled up.

She marches towards them.

EXT. CHURCH GRAVE YARD - DAY

Bertha Manning and Odette continue to chat, not realising a maddened June is heading for them.

ODETTE

Are you gonna do anything?

Bertha Manning laughs.

BERTHA MANNING

I don't need to.

Odette squints at her.

\section{BERTHA MANNING}

It'll just happen cos we're meant

to be together.

Odette nods, pretending to agree - Bertha Manning sees through it.

\section{BERTHA MANNING}

You think I should tell him?

Odette cringes and shakes her head, when June reaches the two girls, and stares at their pulled-up skirts.

JUNE

What in heaven's name is going on?

Nothing.

BERTHA MANNING

Bertha Manning and Odette both pull down their skirts again, which confirms June's suspicions.

June glares at Odette in disgust. 
Get up!

JUNE

June grabs Bertha Manning's wrist, and yanks her up onto her feet.

Ow!

BERTHA MANNING

June points aggressively at Odette.

JUNE

You stay away from my daughter.

June drags Bertha Manning away.

That hurts!

BERTHA MANNING

EXT. SUNNY CLIFF, CHURCH - DAY

June plasters a fake smile on her face, and makes it look like she's lovingly holding Bertha's hand, so the other churchgoers don't suspect anything.

Bertha glances at David and smiles coyly. David doesn't notice.

June guides Bertha into their black car, and slams the door on her.

June hops in the driver's seat, and the car takes off.

EXT. SUNNY CLIFF - DAY

The black car zooms past a row of small shops - where the dairy will be in 1999 - and turns up a street that leads to Seed Road, up on the cliff.

EXT. JAMIESON (1999) - DAY

The cliff is collapsed, and several EMERGENCY WORKERS and LABOURERS are sifting through the debris of the landslide.

A couple of POLICE OFFICERS are milling about, speaking to some of the TOWNSPEOPLE who are hovering around the cordonedoff disaster zone.

Old Odette stands at a distance from everyone else, and watches as one FIREMAN inspects the steadiness of what little remains of the cliff when a bit of fabric beneath a rock catches his eye.

He bends over and pushes the rock aside and uncovers Bertha Jessop's backpack. 


$$
\begin{gathered}
\text { FIREMAN } \\
\text { Mate, check this out. }
\end{gathered}
$$

The co-worker steps over the rocks to look over the fireman's shoulder as he opens the backpack up and sees all of Bertha Jessop's belongings inside - including the now-smashed jar of Trent Jessop's coins.

Odette's squints to see what they're looking at.

The co-worker then looks around, and finds Bertha Jessop's jacket and one of the shoes she was wearing the previous night crushed in the rubble.

The co-worker turns to the other workers and whistles at them.

Odette, recognising the items of clothing, looks completely distraught. She turns away, and scurries over the coast to her house - which was just spared from the landslide.

Meanwhile, a police officer shows a picture of Bertha Jessop to an OLDER TOWNSPERSON who rips the photograph from her.

\section{OLDER TOWNSPERSON}

This is the girl who's missing?

The police officer nods.

$$
\text { You seen her? }
$$

POLICE OFFICER

The older townsperson squints at the photo, disbelieving. OLDER TOWNSPERSON

Thirty odd years ago.

What?

POLICE OFFICER

The older townsperson yells out to his WIFE across the way.

OLDER TOWNSPERSON

Debbie! Will you take a look at this?

Debbie hobbles over to her husband and the police officer.

INT. HOWARD'S HOUSE (1999) - DAY

Bertha Manning (64) is making breakfast in the kitchen when Howard comes in from outside.

Bertha Manning looks angrily at him as she cracks an egg into the frying pan. 
BERTHA MANNING

I told you not to.

Howard shrugs.

HOWARD

I'm only hastening the inevitable.

Howard takes a seat at the table, where he picks up the day's newspaper which has a small article about the landslide on it, with the headline: "Same Time, Same Place".

\section{BERTHA MANNING}

What are you gonna tell her when

she notices her things are missing?

The truth.

HOWARD

Bertha flips the egg in the frying pan, shaking her head to herself.

BERTHA MANNING

This isn't going to be as easy as

you seem to think it's going to be.

INT. HOWARD'S HOUSE, BEDROOM (1999) - DAY

Bertha Jessop is asleep in a comfy bed, in her own room, wearing a brand new nightie.

She wakes to the sound and smell of someone cooking in the kitchen. She stretches out, like a happy cat.

She notices something on her dresser, and gets up to inspect it.

It is a packet of sanitary pads.

Bertha Jessop is mortified at first, but then immensely relieved. She hugs the pads to her chest, and scurries down the hallway, and into the bathroom.

INT. HOWARD'S HOUSE, BATHROOM (1999) - DAY

Bertha flicks the light on, and delights in the clean, luxurious bathroom.

She rips the heavily-stained pad from the gusset of her undies, scrunches it up, and throws it into the bin.

She places a fresh pad in her undies, and breathes a sigh of relief. 
INT. HOWARD'S HOUSE, KITCHEN (1999) - DAY

Bertha Manning is busy cooking pancakes now.

Howard sits at the table, in the adjoining dining room, reading the paper.

The dining table is set up for breakfast - juice, toast, eggs, bacon, and fruit salad.

Bertha Jessop enters.

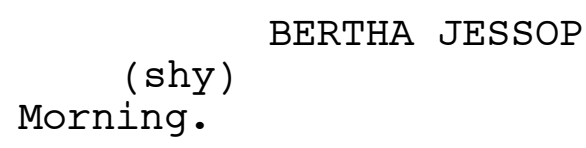

Bertha Manning and Howard turn to Bertha Jessop with huge welcoming grins.

HOWARD

Our wonderful miracle has arisen!

Bertha Jessop, bashful, doesn't know what to do or say.

HOWARD

How did you sleep?

Good, thank you.

BERTHA JESSOP

BERTHA MANNING

Take a seat, sweetie, and help

yourself to breakfast.

Bertha Jessop takes a seat, and stares at all the food in front of her, gobsmacked.

She looks to Howard, who nods at her to serve up.

Bertha Jessop starts loading up her plate, when she sees the article about the landslide in Howard's newspaper.

\section{BERTHA JESSOP}

Did anyone get hurt?

Bertha Manning glares at Howard. Howard puts the newspaper down, and speaks with a serious tone.

HOWARD

Bertha, we want to be honest with you.

Bertha Jessop looks worried.

Bertha Manning watches the conversation, with a look of trepidation. 
HOWARD

You know how everyone thinks

Bertha...

Howard nods his head at Bertha Manning.

HOWARD

...was killed in the first

landslide.

Bertha Jessop nods.

HOWARD

Well, the same thing is going to

happen with you.

Bertha Jessop frowns, not quite understanding.

HOWARD

If it's any comfort, thinking that

you're dead will be easier on your

family in the long run than if they

thought you were missing.

Bertha Jessop looks shocked, now that it's sinking in.

BERTHA JESSOP

I'm never going to see them again?

Bertha Manning sighs, annoyed at Howard's approach. She brings the plate of pancakes to the table. She takes a seat next to Bertha Jessop, and puts her hand on hers.

BERTHA MANNING

The truth is, honey, they never

want to see you again.

Bertha Jessop's lip trembles.

Howard flinches at Bertha Manning's cruelty.

BERTHA MANNING

Your brothers made that very clear

by making that fake birth

certificate, didn't they?

Bertha Manning stares suggestively at Bertha Jessop as she process this.

BERTHA MANNING

Families don't always love each

other.

Tears roll down Bertha Jessop's cheeks. 


\section{BERTHA MANNING}

But we love you, and we're going to

look after you like you deserve to

be looked after.

Bertha Jessop wipes her tears away.

BERTHA MANNING

We're a family now. OK?

Bertha Jessop surveys Howard and Bertha Manning, who smile at her warmly. She nods.

Bertha Manning lets go of Bertha's hand, relieved.

HOWARD

Now, eat up - we've got a big day

of investigating ahead of us.

Bertha Jessop nibbles at the food on her plate.

EXT. JAMIESON, COAST (1999) - DAY

Odette is standing at the edge of the water - wearing every layer of clothing that she owns.

She turns to look at her make-shift house one last time, before stepping into the ocean.

EXT. OCEAN (1967) - DAY

Michael has managed to row to shore, but is now struggling to navigate the crashing waves.

But seeing the Sunny Cliff township in the distance keeps him going.

EXT. MANNING HOUSEHOLD (1967) - DAY

The black car that June Manning was driving is now parked up outside a farmhouse that sits at the top of the cliff.

WILLIAM MANNING (40s) can be seen toiling away on the farm in the distance.

The front door to the house has 20 seed Road written on it the one that Odette will use in the future for her haphazard house.

INT. MANNING HOUSEHOLD (1967) - DAY

Bertha Manning is sitting at the dining table eating a delicious-looking sandwich, when June enters the room with a magazine in hand. 


$$
\begin{gathered}
\text { JUNE } \\
\text { I think you're ready to read this. }
\end{gathered}
$$

June puts the magazine down in front of Bertha Manning.

Bertha Manning stares at it, quizzically. It is a magazine on "How to be a good wife".

JUNE

It worked on your father.

June goes into the adjoining kitchen, and slices into a cake.

Bertha Manning opens the magazine, and reads curiously.

June brings Bertha Manning a slice of cake. Bertha gobbles it down, barely thinking about it.

$$
\begin{aligned}
& \text { JUNE } \\
& \text { Are there any boys at school that } \\
& \text { you like? }
\end{aligned}
$$

Bertha Manning scrunches up her face, her mouth full of cake.

$$
\text { BERTHA MANNING }
$$

Yuck, no. Boys are gross.

June looks very concerned.

$$
\text { JUNE }
$$

What about Jimmy Barker from Sunday School?

Bertha laughs.

$$
\text { BERTHA MANNING }
$$

He already goes around with

Elizabeth Hobson.

June seems surprised by this.

$$
\text { JUNE }
$$

You're much prettier than Elizabeth

Hobson.

Bertha Manning shoves some more cake into her gob.

\section{I know.}

$$
\text { BERTHA MANNING }
$$

Bertha Manning goes back to flicking through the magazine and lands on a page about "How to attract a husband". She reads it intensely.

$$
\text { JUNE }
$$

It's about time you put those good looks to use. 
June stands up, and straightens out her apron.

JUNE

I think I'll put the word out that you're on the lookout for a nice young man.

Bertha Manning doesn't seem to hear this as she continues to read the magazine.

June flips through her address book, and picks up the phone.

EXT. SUNNY CLIFF, COAST (1967) - DAY

The coast is clear of the debris, and Odette's ramshackle house.

Michael makes his way onto shore, but is completely drenched and exhausted.

He drags his rowboat onto the rocks, then makes his way towards the township.

EXT. ODETTE'S HOUSE, JAMIESON (1999) - DAY

Father David is standing outside Odette's house, knocking on the door. He is exhausted, like he's been at this a while.

FATHER DAVID

Odette! I know you're in there!

There is no response.

FATHER DAVID

You need to talk to the police!

Father David, waning, knocks again.

FATHER DAVID

Think of that poor girl's family!

Still nothing.

Father David, concerned now, pushes Odette's door open and creeps inside.

INT. ODETTE'S HOUSE, JAMIESON (1999) - DAY

Father David steps into Odette's ramshackle house - it's empty.

But there is a note on her coffee-table, addressed to Father David and begins: "It is quite obvious that I have been cursed for past misdeeds. I can no longer bear the burden..." 
As Father David continues to read the letter, a grave expression comes over his face. He scrunches the note up, and runs outside.

EXT. COAST, JAMIESON (1999) - DAY

Father David clambers over the rocks towards the ocean.

When he reaches the edge of the water, he surveys the area until he sees Odette's limp body being thrashed around by the waves.

Father David rips the note up, and tosses the bits into the water, before peeling his clothes off and wading into the sea to retrieve Odette's body.

EXT. HOWARD'S HOUSE (1999) - DAY

Howard guides Bertha Jessop to a shed at the back of the house.

In the daylight Bertha Jessop sees that Howard's property is completely enclosed by a high fence lined with barbed wire, and concealed, from the outside, by large pine trees.

Howard opens the tin shed and lets Bertha Jessop in.

INT. HOWARD'S SHED (1999) - DAY

Howard's shed is set up like a laboratory.

A work bench lines every wall, and is cluttered with testtubes, animal skins, dead insects pinned onto corks, unruly plants, weird-looking equipment, papers and charts.

A framed picture of each Bertha, at a different age, hangs on each wall.

In the middle of the room is a desk with a typewriter on it, and boxes of files stored underneath.

A chair sits opposite the desk, as if it's been set up for an interview.

Bertha Jessop takes a look at all the Berthas on the wall.

$$
\text { BERTHA JESSOP }
$$

These are seriously real people?

HOWARD

As real as you are.

This comment makes Bertha Jessop look uncomfortable.

Howard retrieves a clear-file folder from his desk. 


\title{
HOWARD
}

I've collated all the recorded

meetings of past lookalikes that I

could find.

Howard hands the folder over to Bertha who flicks through it, and sees pairs of people who look alike.

HOWARD

There aren't too many, but you can see they all come in pairs.

Bertha Jessop gets to the end of the doppelgangers, and closes the clear file.

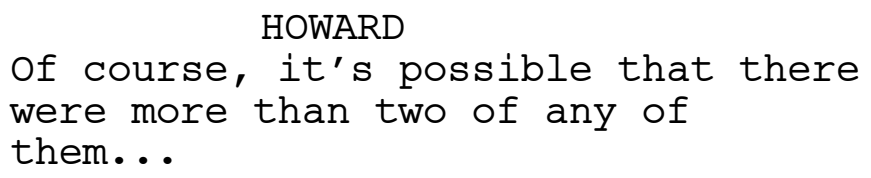

Howard takes the clear file from Bertha Jessop.

\section{HOWARD}

...but they just never met because

of how vast and messy the world is.

Howard puts the clear file away, and takes a seat behind his desk. He watches Bertha inspecting all the junk spread out on his work bench.

\section{HOWARD}

My current theory is that we all

have doppelgangers, but it's just

that our paths never cross. Which

is why the case of you and the

other Berthas is so remarkable.

Bertha Jessop turns back to Howard.

\section{HOWARD}

I think the thing that's pulled you

all together is a microcosm of the

design of the universe itself.

Bertha Jessop looks utterly confused.

\author{
HOWARD \\ So once I've figured out the \\ design, I'll - we'll - be able to \\ predict the future!
}

Howard is wide-eyed with excitement, while Bertha looks like she's about to faint.

Howard guides her to the chair in the middle of the room.

Bertha flops down on the chair, and tensely taps both sides of the chair with her fingers over and over again. 
Are you OK?

Bertha can't stop.

$$
\begin{gathered}
\text { HOWARD } \\
\text { What are you doing? }
\end{gathered}
$$

Howard looks panicked.

$$
\begin{aligned}
& \text { HOWARD } \\
& \text { How about we ease into things by } \\
& \text { verifying some information? }
\end{aligned}
$$

Bertha keeps tapping.

Howard feeds a bit of paper into the typewriter on his desk, and types in "Bertha" before pausing.

HOWARD

No middle name, is that right?

Bertha stops tapping.

$$
\text { BERTHA JESSOP }
$$

The day that my brothers made that

birth certificate they also tricked

me into walking under a ladder.

Could that have something to do

with all this?

Howard considers this seriously.

$$
\begin{aligned}
& \text { HOWARD } \\
& \text { I'm not usually one for } \\
& \text { superstition, but nothing can be } \\
& \text { ruled out. }
\end{aligned}
$$

Howard writes this down on a bit of paper on his desk.

$$
\text { HOWARD }
$$

The ladder from your bunk bed?

Bertha Jessop looks freaked out.

Yeah?

$$
\text { BERTHA JESSOP }
$$

Howard regrets his words, and smiles to smooth it over.

$$
\begin{aligned}
& \text { HOWARD } \\
& \text { Just a hunch. }
\end{aligned}
$$

Howard writes another note, before returning to his typewriter. 


\section{HOWARD}

Let's get back to the beginning

again. No middle name?

Bertha Jessop, tentatively shakes her head.

Howard continues to type "Bertha Jessop: Interview One (25.06.1999)".

Howard pushes the typewriter's platen to the side to start a new line.

\section{HOWARD}

Date of birth is sixteenth of January, 1986?

Bertha Jessop nods.

Howard types.

HOWARD

And you were born at Waikato Hospital?

Bertha Jessop hesitates, but nods.

Howard types.

\section{HOWARD}

And your mother died of respiratory problems shortly after giving birth to you?

Bertha Jessop tenses up.

\section{BERTHA JESSOP}

How do you know all this stuff?

HOWARD

There's certain information I've

had to acquire for the purposes of

this investigation.

BERTHA JESSOP

That I sleep in a bunk?

Howard sighs, but sounds proud of his dedication.

HOWARD

I've been tracking you since you

were entered on the birth registry,

Bertha.

Tracking me?

BERTHA JESSOP

HOWARD

I mean - observing you from afar. 


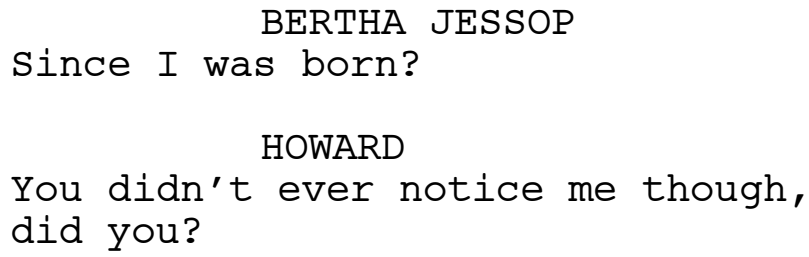

Bertha Jessop subtly surveys Howard up and down, her concern intensifying.

$$
\text { HOWARD }
$$

Well, there was that one time when

some boys from your school were

hassling you and I stepped in.

Howard winks at Bertha Jessop, not realising how freaked out this is making her.

$$
\begin{aligned}
& \text { BERTHA JESSOP } \\
& \text { That was you? }
\end{aligned}
$$

Howard nods. Bertha starts frantically tapping her fingers against the side of the chair again.

\section{HOWARD}

Perhaps we should get to the stuff

I'm a bit more hazy about. What's

your earliest memory?

Bertha Jessop continues tapping while staring down at her feet.

HOWARD

What about dreams? Any recurring

dreams?

Howard waits for Bertha Jessop to respond.

BERTHA JESSOP

I don't want to do this anymore.

Howard frowns, concerned.

\section{Do what?}

HOWARD

Bertha Jessop stands up, and bursts out the garage door.

Howard follows after her.

HOWARD

I don't think you understand how

big this thing is, Bertha! 
EXT. HOWARD'S HOUSE (1999) - DAY

Bertha Jessop runs for the gate, with Howard chasing after her.

Bertha Jessop tries to yank the gate open but sees that it's locked shut with a heavy-duty padlock. Bertha Jessop's panic intensifies.

$$
\text { I want to go home! }
$$

Howard approaches her, but stands at a distance.

HOWARD

This is your home.

Bertha Manning steps out of the house, and walks towards them.

Bertha Jessop scours the fence-line for another way out - to no avail. She drops to the ground and starts digging under the fence.

$$
\text { You can't defy fate, Bertha. }
$$

Bertha Manning stands next to Howard, and they watch her dig.

Bertha Jessop is disheartened to see that the fence posts are deeply embedded in the soil.

Bertha Jessop looks up at the top of the fence, lined with barbed wire.

She stands up, takes a deep breath, and climbs onto the fence, clumsily clinging to the horizontal fence rails.

$$
\begin{aligned}
& \text { BERTHA MANNING } \\
& \text { (to Howard) } \\
& \text { You went too hard too soon. She's } \\
& \text { not a bloody robot. }
\end{aligned}
$$

Bertha Manning glares at Howard, then back at Bertha Jessop who is making surprisingly good progress at climbing.

\section{BERTHA MANNING}

For Christ's sake.

Bertha Manning goes back into the house.

Bertha Jessop reaches the top of the fence, but has nothing to hold onto except for the barbed wire.

She rolls her sleeves down over her hands, and tries to gently pry the barbed wire away but the barbs cling onto the fabric and she becomes stuck. 
As she tries to free herself, Bertha Manning returns from the house with a tranquilizer gun in hand.

Bertha Manning aims it at Bertha Jessop, and shoots.

The dart hits Bertha Jessop in the back of her right leg, and she immediately falls to the ground with a thud and a crunch.

EXT. SUNNY CLIFF, TOWNSHIP (1967) - DAY

Michael, bedraggled, heads into the township and tries to approach various people who either look upon him with scorn or ignore him completely.

He heads into the grocery store (which will one day become the Jamieson dairy).

INT. SUNNY CLIFF, SCHMIDT'S GROCERY STORE (1967) - DAY

A bell jingles as Michael enters the store. The FEW PEOPLE who are inside the store try their best not to stare at Michael who looks deranged.

MR. SCHMIDT (50s) is standing behind the counter.

MR. SCHMIDT

Can I help you?

Michael speaks with an Australian accent.

MICHAEL

I'm looking for a place to stay the night?

Mr. Schmidt eyes Michael up and down.

MR. SCHMIDT

Sunny Cliff's not really a tourist

destination...

Michael deflates, and looks like he might cry.

MICHAEL

I've just escaped the cruise ship I

was working on cos some of the

passengers contracted a deadly

rash.

Everyone in the store has stopped pretending not to listen.

MICHAEL

I don't have anywhere to go.

Michael holds his arms out. 
MICHAEL

I don't have the rash myself - you

can check me.

Mr. Schmidt looks embarrassed and guilty.

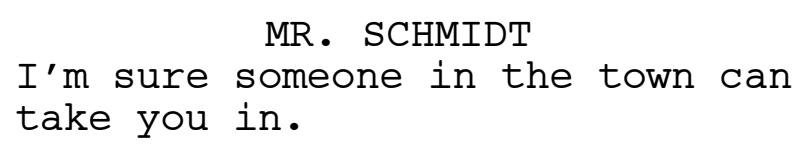

At that, the people in the store bow their heads and scurry

as fast as they can outside.

Mr. Schmidt smiles at him.

$$
\begin{gathered}
\text { MR. SCHMIDT } \\
\text { (clearly lying) } \\
\text { I'd take you in, but we've no room. }
\end{gathered}
$$

Michael deflates again.

MR. SCHMIDT

I'll make some calls for you, if you'd just wait outside.

Michael looks grateful.

Thank you .

MICHAEL

Mr. Schmidt smiles, and Michael takes a seat outside.

Mr. Schmidt picks up his phone.

INT. BERTHA MANNING'S BEDROOM (1967) - DAY

Bertha Manning is lying on her bed in her messy room, reading the magazine and taking down notes in her notebook.

The phone can be heard ringing in the kitchen. When it stops, Bertha puts down her notebook and we now see that her notebook reads:

How to woo Father David:

- Sew buttons onto a shirt in his presence.

- Eat delicately around him.

- Ask him about his day.

- Look nice for him.

Bertha Manning gets up and retrieves a set of paints from under her bed. 
She stands in the front of her dressing-table mirror and proceeds to apply paint to her face as if it was make up.

INT. MANNING HOUSEHOLD, KITCHEN (1967) - DAY

June is on the phone, looking animated.

$$
\begin{aligned}
& \text { JUNE } \\
& \text { How old is he? } \\
& \text { MR. SCHMIDT (PHONE) } \\
& \text { Uh - I'd say in his twenties? }
\end{aligned}
$$

June bobs her head side to side, weighing something up.

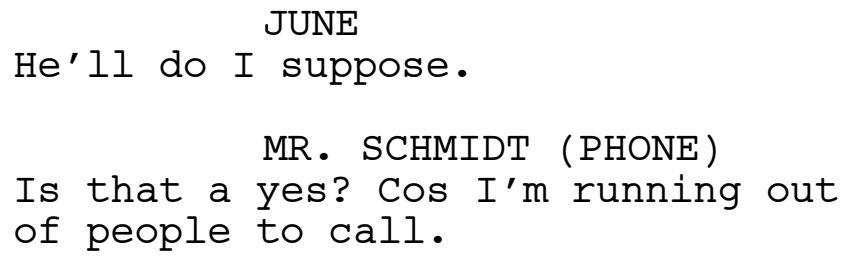

June pauses.

$$
\begin{aligned}
& \text { JUNE } \\
& \text { I'll be right over. }
\end{aligned}
$$

June hangs up the phone, excited. She heads down the hallway.

INT. MANNING HOUSEHOLD, HALLWAY (1967) - DAY

June pushes open Bertha Manning's closed-bedroom door without knocking.

JUNE

Fate has delivered you a suitor!

INT. BERTHA MANNING'S BEDROOM (1967) - DAY

June stands in the doorway, horrified to see that her daughter has paint all over her face, and looks a bit like a clown.

$$
\begin{gathered}
\text { JUNE } \\
\text { What on earth are you doing? }
\end{gathered}
$$

Bertha Manning doesn't seem perturbed.

BERTHA MANNING

Making myself look nice.

Bertha strikes a pose to show off her "make up". June shakes her head. She grabs her wrist. 


$$
\text { Absolutely not. }
$$

June drags her daughter out of her room.

INT. MANNING HOUSEHOLD, HALLWAY (1967) - DAY

June shoves Bertha Manning into the bathroom.

JUNE

I want it cleaned off by the time

I've returned.

INT. MANNING HOUSEHOLD, BATHROOM (1967) - DAY

Before Bertha Manning can respond, June slams the door.

Bertha Manning looks at herself in the mirror and likes what she sees.

Bertha Manning waits to hear her mother closing the front door and starting the car engine, then heads down the hallway towards the kitchen.

INT. MANNING HOUSEHOLD, KITCHEN (1967) - DAY

Bertha Manning creeps into the kitchen, and flicks through June's address book until she finds Father David's number.

She dials the number, and waits for Father David to pick up.

FATHER DAVID (PHONE)

Good Afternoon, this is Father

David speaking.

Bertha Manning becomes flustered and nervous.

BERTHA MANNING

Hello, Father David.

There is a pause.

FATHER DAVID (PHONE)

Who am I speaking with?

Bertha Manning giggles awkwardly .

BERTHA MANNING

It's Bertha Manning.

FATHER DAVID (OVER PHONE)

Oh. Miss Manning. How can I help? 
Bertha Manning coyly twists the phone cord around her fingers, but soon gets tangled and loses circulation in her fingers.

She untangles herself, frustrated.

BERTHA MANNING

I was wondering if we could talk some time?

FATHER DAVID (PHONE)

Oh, yes? About what?

BERTHA MANNING

You know...just things.

Bertha Manning giggles again.

FATHER DAVID (PHONE)

I'm afraid I'll need you to be a

bit more specific.

Bertha Manning pauses to think.

BERTHA MANNING

I have a confession I need to make.

Bertha Manning grins, pleased with herself.

FATHER DAVID (PHONE)

I don't conduct confessionals,

Bertha.

Oh.

BERTHA MANNING

Bertha Manning frowns, frustrated.

BERTHA MANNING

But it's really important and I can only talk to you about it.

She hears Father David sighing.

FATHER DAVID (PHONE)

I'll be at the church from about

six, if you want to drop by.

Bertha Manning beams with excitement.

It's a date!

BERTHA MANNING

Bertha Manning hangs up, and does a little celebratory dance in the kitchen. 
EXT. JESSOP HOUSE (1999) - DAY

A police car pulls up in the Jessop's driveway. Jake, Callum, and Brett step out of the backseat - they're completely wrecked.

A policeman - OFFICER SAMSON - steps out of the drivers seat, and follows the brothers to the front door.

INT. JESSOP HOUSE, LOUNGE (1999) - DAY

Trent is sitting on the couch, silently fuming.

Kyle lies in the middle of the floor stabbing a dead

cockroach with a pen. The brothers tentatively head inside.

Trent stands up, and marches towards the brothers - ready to clobber them over the head, when officer Samson enters, and he has to restrain himself.

TRENT
For fuck's sake.

Callum bursts into tears.

TRENT
You don't get to cry.

Officer Samson tries his best to diffuse the tension.

OFFICER SAMSON

You must be Trent.

Trent nods. Officer Samson shakes Trent's hand.

OFFICER SAMSON

Officer Samson. Mind if we take a seat?

Trent leads Officer Samson into the lounge.

Trent lightly kicks Kyle to get him to sit up.

All six of them take a seat.

Officer Samson clears his throat.

OFFICER SAMSON

We've just had word that they've

found Bertha's backpack, and some

items of clothing, in the rubble of

the landslide.

Callum is trembling so Jake puts his arm around him. 


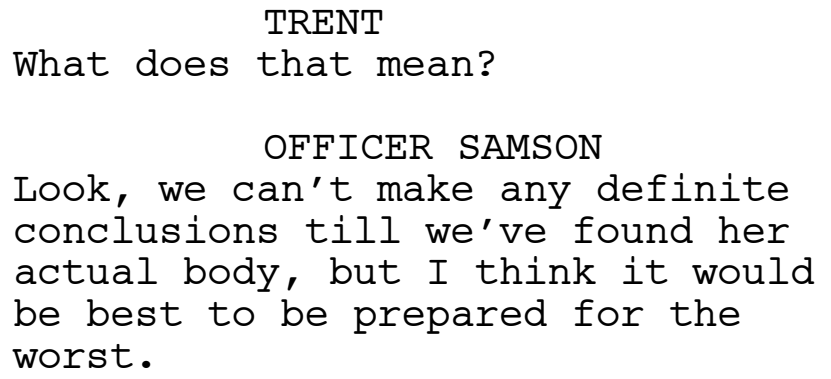

The Jessops sit in tense silence, until Callum can't bear it any longer and storms out of the room.

INT. JESSOP HOUSE, BEDROOM (1999) - DAY

Callum is lying on Bertha's top bunk when Jake comes into the room.

Callum remains lying on his back as he speaks.

$$
\text { CALLUM }
$$

This isn't my fault.

Jake bristles.

$$
\begin{gathered}
\text { JAKE } \\
\text { I never said it was. }
\end{gathered}
$$

Callum stares up at the ceiling.

\section{CALLUM}

You reckon it's cos she walked

under that ladder?

Callum turns his head and sees that Jake is frowning.

Callum resumes staring at the ceiling, his eyes prick with tears.

Jake shakes his head.

JAKE

I don't reckon she's dead.

Callum sits up.

\section{CALLUM}

How do you know?

Jake shrugs.

$$
\text { I just have this feeling. }
$$

Callum shrinks again. 
Oh.

Callum lies back down, disappointed.

Jake looks annoyed that Callum doesn't believe him.

\section{JAKE}

We better make sure Dad's not

murdered anyone out there.

Callum doesn't move or say anything.

Jake slinks out of the room on his own.

INT. MANNING HOUSEHOLD, DINING ROOM (1967) - DAY

Bertha Manning is sitting at the dining table, she has not cleaned the paint off her face and she now wears one of her mother's frocks, which is anything but the right fit for her.

She is furiously trying to sew a button onto a man's shirt, while trying to delicately eat another piece of cake.

\section{BERTHA MANNING \\ How was your day?}

Bertha Manning is pretending to listen to the response, when June opens the front door and invites Michael in.

June looks horrified when sees the state her daughter is in.

$$
\text { Bertha, darling! }
$$

Bertha Manning looks up and sees Michael standing there. She's not embarrassed at all, even though Michael is staring at her like he's seen a ghost.

$$
\text { JUNE }
$$

This is Michael. Michael this is my lovely daughter, Bertha.

Bertha?

$$
\text { MICHAEL }
$$

Michael staggers backwards a little.

$$
\text { Oh, are you OK? }
$$

June holds her hand out for him, but he doesn't take it.

Michael doesn't take his eyes off Bertha Manning.

June is pleased with his apparent interest in her daughter. 
Isn't it a beautiful name? It just

came to me when she was born, like

someone was whispering it in my

ear.

Bertha Manning starts to get creeped out by Michael's staring, but, figuring her make-up must be working, she flicks her hair back confidently.

JUNE

Take a seat, and I'll rustle

something up for you. You must be

famished.

Michael tries to compose himself.

MICHAEL

Thank you.

Michael takes a seat opposite Bertha Manning, and tries not to look at her as she continues to sew the button on the shirt while nibbling delicately on the cake.

INT. HOWARD'S HOUSE, LOUNGE (1999) - DAY

Bertha Jessop is sleeping on the couch.

Bertha Manning is sitting on the floor next to her, gently pressing a cold flannel against her head.

Bertha Jessop starts to wake, so Bertha Manning drops the flannel and forces herself to cry.

She holds onto Bertha Manning's hand

\section{Bertha?!}

BERTHA MANNING

Bertha Jessop is groggy and disoriented.

Bertha Manning bursts into uncontrollable sobs, and gives Bertha Jessop an affectionate hug.

\section{BERTHA MANNING}

We thought we'd lost you!

Bertha Jessop strains to recall.

BERTHA MANNING

Please don't scare us like that

again, Bertha.

Bertha Manning holds onto Bertha Jessop's hand.

Bertha Jessop is taken aback by Bertha Manning's emotion. 


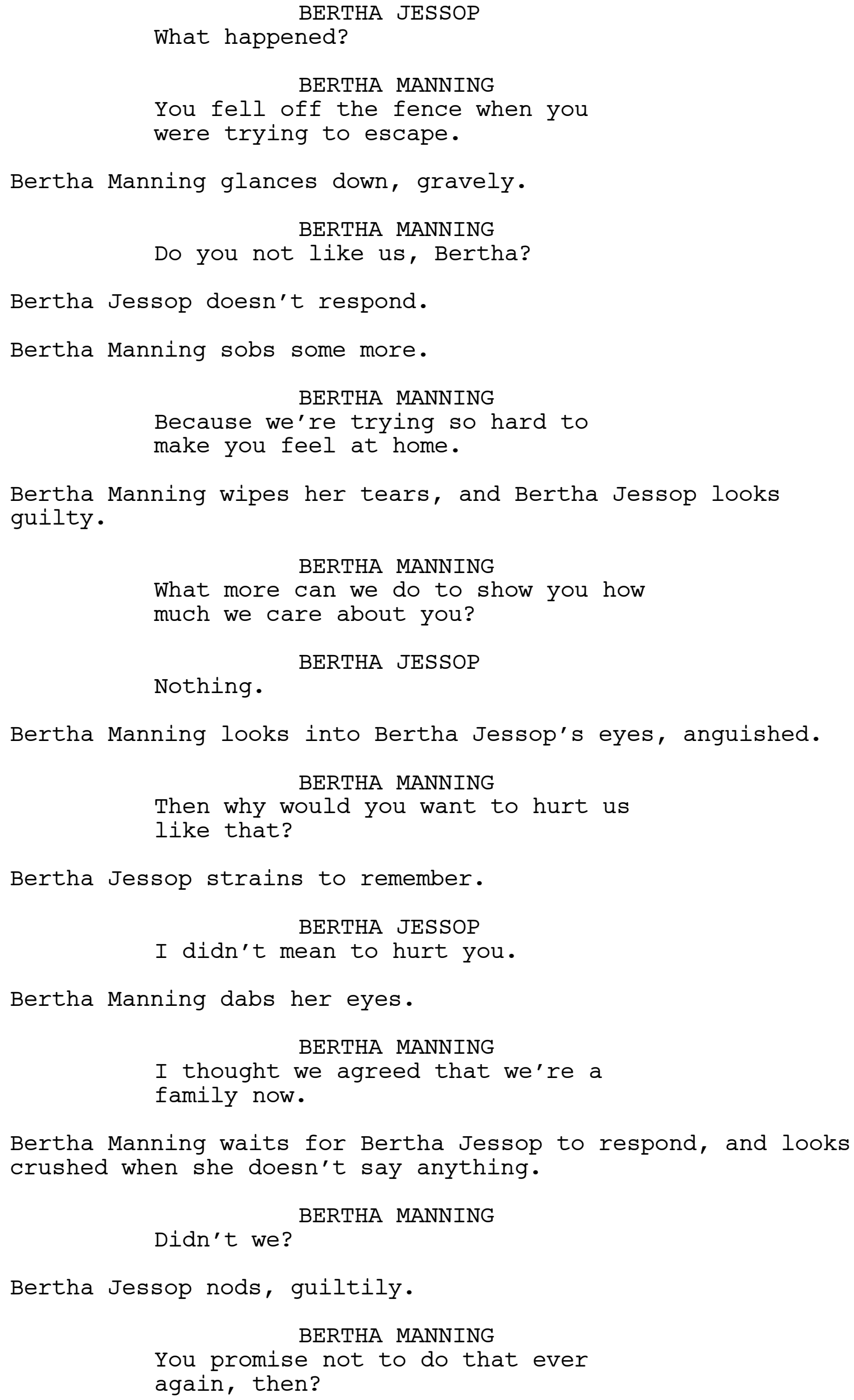


Bertha Jessop nods again.

\title{
Sorry.
}

BERTHA JESSOP

Bertha Manning rubs the top of Bertha Jessop's head.

That's OK.

$$
\text { BERTHA MANNING }
$$

The two fall silent for moment.

\section{BERTHA MANNING}

How about we play a card game or something? As a family?

Bertha Jessop likes the sound of this.

INT. MANNING HOUSEHOLD, BERTHA'S BEDROOM (1967) - DAY

Bertha Manning, still wearing the dress and paint-makeup and carrying the man's shirt, opens her bedroom window and starts to make her way through it when there is a knock on the door.

Bertha Manning frowns. She goes to the door, and opens it.

She rolls her eyes when she sees Michael there.

\author{
BERTHA MANNING \\ Should have realised it was you - \\ mum never knocks.
}

Bertha Manning lets Michael into her room.

Michael stands a few paces away, staring at Bertha in awe.

BERTHA MANNING

Why do you keep looking at me like

that?

Michael realises he's being creepy, and briefly averts his eyes. But he can't resist staring for long.

MICHAEL

Do you remember me?

Bertha Manning laughs, but stops when he sees how earnest he's being.

From where?

BERTHA MANNING

Tears fill Michael's eyes.

Are you OK?

BERTHA MANNING 
Michael wipes the tears away, but they just come thicker and faster.

\author{
MICHAEL
}

Are you OK? Are you happy?

Bertha Manning gives this some genuine thought.

BERTHA MANNING

I mean, things could be better.

MICHAEL

Yeah? That woman isn't good to you?

Who? My mum?

BERTHA MANNING

MICHAEL

I guess she is your mum, yeah.

Bertha Manning frowns.

BERTHA MANNING

She's kind of a drag, actually.

Especially today.

Michael kneels down on the floor and looks into Bertha's eyes.

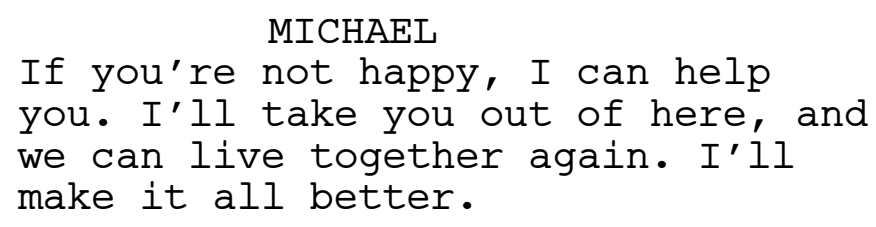

Bertha Manning looks disturbed.

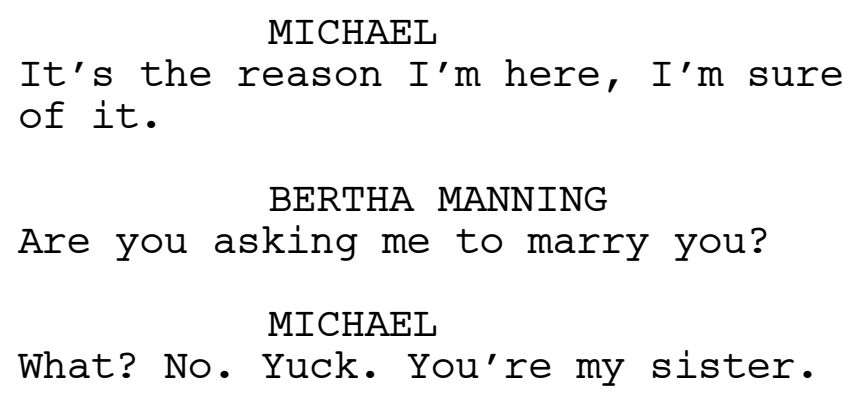

Michael delves into his pocket. 


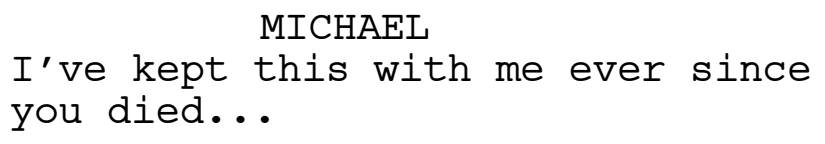

Michael pulls out a locket.

Died?

\section{BERTHA MANNING}

Michael flicks the locket open and shows it to Bertha Manning.

Inside is the portrait of a young girl - Bertha Greive - in 1959 who looks exactly like Bertha Manning. The girl is sitting next to Michael as a ten-year old boy.

Bertha Manning takes the locket from Michael, completely dumbfounded.

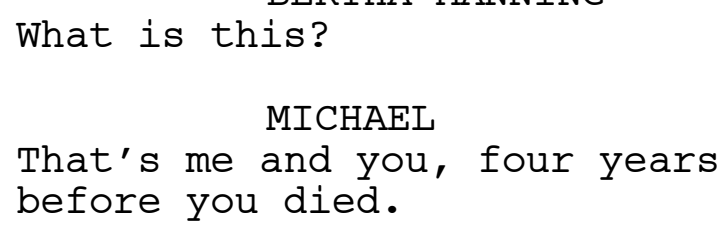

Michael shakes his head, disbelieving.

\section{MICHAEL}

Those people on the ship all had

the same rash that killed you.

That's why I escaped. And then I

ended up here with you.

BERTHA MANNING

You're saying I'm your sister's ghost?

June's footsteps can be heard approaching Bertha Manning's bedroom, and she soon appears in the doorway and is shocked to see Michael down on one knee in front of her daughter.

\section{Oh!}

JUNE

Michael stands up, embarrassed.

MICHAEL

It's not what it looks like.

June can't help but grin a little bit.

JUNE

What does it look like?

Michael turns to Bertha Manning. 


$$
\text { Maybe a reincarnation? }
$$

Bertha Manning frowns, confused.

Michael scurries past June and out the door.

JUNE

I can't say I'm not relieved, but

please remember that you're a lady.

Bertha Manning doesn't move or say anything.

JUNE

Bertha?

June sees the locket.

$$
\text { What's that? }
$$

\title{
JUNE
}

June leans over to grab the locket, but Bertha Manning snaps it shut.

\section{BERTHA MANNING}

Bertha Manning shoves June out the door, and slams it on her.

Bertha Manning opens the locket again, and stares at the picture of her doppelganger.

She snaps the locket shut again, then proceeds to wipe the paint off her face, and change into clothes that actually fit her.

She leaves the man's shirt behind, and climbs through the open window.

INT. HOWARD'S HOUSE, LOUNGE (1999) - NIGHT

Bertha Jessop, Bertha Manning and Howard are sitting around the coffee table in lounge - laughing raucously as they play a game of snap.

Bertha Jessop slams her hand down on the big pile of cards.

\section{Snap!}

BERTHA JESSOP

Howard and Bertha Manning groan, jovially, as Bertha Jessop pulls the cards towards - sealing the win.

\author{
BERTHA MANNING \\ You're a quick one!
}


Bertha Jessop smiles, and knocks the set of cards against the coffee table until they're in a perfectly neat pack.

Howard holds out his hand for the cards.

HOWARD

Let me show you a trick.

Bertha Jessop hands the cards over.

Howard holds out the cards out in a fan.

HOWARD

Pick a card, any card, but don't

let me see it.

Bertha Jessop scrunches her face up.

BERTHA JESSOP

I know that one.

HOWARD

Oh?

BERTHA JESSOP

Yeah. My brothers taught me. You

split the deck to see the card it's

next to when I put it back.

Howard raises his eyebrows, and persists.

HOWARD

Pick a card.

Bertha Jessop rolls her eyes, and picks a card.

Bertha Jessop keeps her eye on Howard while she very quickly glances at the card - it's the six of hearts.

Howard doesn't split the deck of cards. He puts it down on the table.

HOWARD

Put the card anywhere in the deck.

Howard closes his eyes, and Bertha Jessop slips the card back in the deck.

HOWARD

Now shuffle them.

Bertha Jessop shuffles the cards, and puts them down again.

Howard opens his eyes, picks up the deck of cards, searches through them and picks out the six of hearts and shows it to Bertha Jessop.

Bertha Jessop is impressed but skeptical. 


\section{BERTHA JESSOP}

How did you do that?

Howard glances at Bertha Manning to see if he's allowed to tell her. Bertha Manning nods.

\section{HOWARD}

I've learnt how to predict certain things based off of my findings.

Bertha Jessop is stunned.

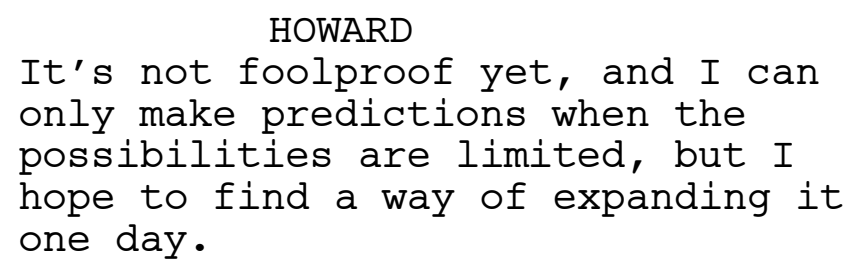

Bertha Jessop looks to Bertha Manning to see if he's having her on.

BERTHA MANNING

Pretty cool, huh?

Bertha Jessop nods.

EXT. SEED ROAD (1967) - EVENING

Bertha Manning power-walks down seed Road as rain begins to drizzle.

EXT. SUNNY CLIFF, TOWNSHIP (1967) - EVENING

Bertha Manning walks past the shops, which are all closed now. The rain sets in harder.

EXT. FATHER DAVID'S HOUSE (1967) - EVENING

Bertha Manning stands under the awning of Father David's front porch. She knocks continuously.

Eventually Father David answers the door, looking dishevelled.

He is perplexed, and annoyed, to see Bertha Manning standing there.

He furtively closes the door behind him, and steps onto the porch. 
BERTHA MANNING

I know, I just really needed to

talk to you.

Bertha Manning bursts into tears, and clings on to David in a one-sided hug.

David peels Bertha off of him.

$$
\begin{aligned}
& \text { FATHER DAVID } \\
& \text { What's happened? }
\end{aligned}
$$

Bertha sniffs.

BERTHA MANNING

I'm in love with you, Father David.

Father David is baffled.

BERTHA MANNING

And I know you feel the same way about me.

Father David laughs at the absurdity.

FATHER DAVID

That's most certainly not the case.

Bertha Manning tries to ignore this comment.

BERTHA MANNING

Well it's not going to work out

anymore because I just found out

I'm a ghost.

Father David squints at Bertha Manning, disbelieving.

BERTHA MANNING

I knew I was different from

everyone else, but I don't wanna be

this kind of different.

Father David shakes his head, irate.

BERTHA MANNING

I don't know what to do.

Bertha Manning's lip quivers.

FATHER DAVID

You're a spoilt brat, Bertha. And

you need to learn that the world

doesn't revolve around you.

Bertha Manning staggers backwards - this isn't how she thought this would go. 
FATHER DAVID

I know that you think you're better

than everyone else, but people who

are truly better than other people

don't go around bragging about it.

BERTHA MANNING

I'm not bragging. Look.

Bertha Manning pulls out the locket and shows him the

picture. Father David looks at it but doesn't seem impressed.

FATHER DAVID

I'm not interested in your games,

Bertha.

Bertha is completely deflated.

FATHER DAVID

Go home, and try to put other

people's needs before your own.

Father David opens the door and Bertha Manning gapes when she catches a glimpse of Odette, wrapped in an eiderdown, and standing in Father David's hallway.

Bertha Manning steps forward to get a better look, but Father David closes the door on her.

Bertha, stunned, creeps over to the front window and peers in - she's so distracted by what she sees that she doesn't seem to notice that she's getting drenched by the rain.

INT. FATHER DAVID'S HOUSE (1967) - EVENING

Odette is standing in the hallway, naked except for the duvet around her.

ODETTE

Did she know I was here?

FATHER DAVID

I hope not.

Father David looks immensely guilty and flustered, and ushers Odette into the adjoining bedroom.

INT. FATHER DAVID'S HOUSE, BEDROOM (1967) - NIGHT

Odette takes a seat on Father David's bed, pensive.

Father David picks up her clothes which are scattered over the floor, and throws them over to her.

FATHER DAVID

You need to go. 
Odette looks crushed, but it comes out as anger. She puts her clothes on, then storms out of the room, without saying a word to Father David.

Father David flinches as he hears the door slamming.

EXT. FATHER DAVID'S HOUSE (1967) - NIGHT

Bertha Manning tries to duck out of sight as Odette marches past, but Odette spots her, panicked.

\section{ODETTE}

Bertha!

Bertha Manning sprints off down the street.

INT. HOWARD'S HOUSE (1999) - NIGHT

The house is quiet and dark.

INT. HOWARD'S HOUSE, BEDROOM (1999) - NIGHT

Bertha Jessop is sitting up in bed, reading over her fake birth certificate.

She pulls herself up from the bed, and creeps out into the hallway.

INT. HOWARD'S HOUSE, FRONT DOOR (1999) - NIGHT

Bertha Jessop quietly opens the front door, and creeps outside.

EXT. HOWARD'S HOUSE, SHED (1999) - NIGHT

Bertha Jessop makes her way into Howard's shed.

INT. HOWARD'S SHED (1999) - NIGHT

Bertha Jessop flicks the light on, and heads over to Howard's desk.

She pulls out all the boxes of files, which have been labelled and organised by each Bertha.

Bertha Jessop proceeds to rummage through the boxes - pulling out papers and spreading them across the floor.

INT. MANNING HOUSEHOLD, DINING ROOM/ KITCHEN (1967) - NIGHT

The dining table has been set for dinner. 
Michael and WILLIAM MANNING (40s - gruff, and wearing his dirty farming clothes) are sitting at the table in awkward silence, while June prepares dinner in the kitchen.

JUNE

Would you mind giving me a hand, Michael?

Michael, relieved for something to do, joins June in the kitchen.

June grins.

JUNE

You're such a gentleman.

She nods to the clumpy gravy that is bubbling away on the stove.

JUNE

If you could give that a stir.

June hands Michael a wooden spoon, but when he goes to take it he gasps and drops the spoon on the floor.

What is it?

Michael stares at his hand, before trying to conceal it with his sleeve. But June has seen what's caught his eye - a red, bumpy rash is covering the entire upper-side of his hand.

June pushes him away from the food, grabs a pair of gloves from under the sink and puts them on.

$$
\begin{gathered}
\text { JUNE } \\
\text { I trained as a nurse. }
\end{gathered}
$$

She pulls Michael's sleeve up and they see that the rash has spread up his arm.

Michael starts to hyperventilate.

June squints at it, confused.

$$
\text { JUNE }
$$

This is the deadly rash that you

said broke out on the cruise ship?

Michael nods, petrified. June drops his hand.

JUNE

That's just ring worm.

Michael frowns, confused. 
JUNE

It's not pleasant by any means, but

definitely not fatal.

Michael stares at his arm.

\section{MICHAEL}

But my sister died from this.

June frowns.

JUNE

That doesn't sound right to me.

Michael stares at June, confounded.

JUNE

We've some ointment in the bathroom

cabinet if you'd...

Michael scurries out of the room and down the hall.

June is a bit taken aback. She looks to her husband in the adjoining dining room.

JUNE

$$
\begin{aligned}
& \text { Maybe he's a bit too odd to be our } \\
& \text { son-in-law. }
\end{aligned}
$$

At that, Bertha Manning bursts through the front door drenched, dishevelled, and tears pouring down her face.

June turns to her, stunned.

$$
\begin{gathered}
\text { JUNE } \\
\text { Where have you been?! } \\
\text { BERTHA MANNING } \\
\text { Odette's a lying whore! }
\end{gathered}
$$

Bertha Manning storms through the dining room and down the hallway.

June glances at William, gravely concerned, before following after her daughter - forgetting about the gravy which continues to bubble.

William sees that it needs tending to, but doesn't do anything about it.

INT. MANNING HOUSEHOLD, HALLWAY (1967) - NIGHT

Bertha Manning disappears into her room, leaving June out in the corridor. 
INT. MANNING HOUSEHOLD, BERTHA'S BEDROOM (1967) - NIGHT

Bertha Manning has shoved her chair under the door knob, and is lying on her bed, sobbing.

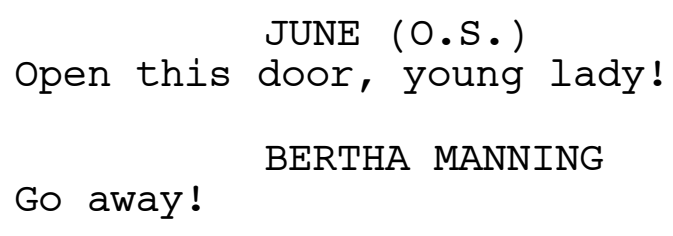

Bertha Manning buries her head in her pillow, when she hears the doorbell ringing.

\section{June! Door!}

WILLIAM (O.S.)

Bertha Manning is relieved to hear her mother's footsteps disappear.

INT. MANNING HOUSEHOLD, ENTRANCE (1967) - NIGHT

The doorbell continues to buzz, as June opens the door.

She is horrified to see odette standing there, drenched in rain and tears streaming down her face just like her daughter.

$$
\text { I need to talk to Bertha. }
$$

June glares at Odette.

$$
\begin{aligned}
& \text { JUNE } \\
& \text { My daughter has no interest in } \\
& \text { talking to the likes of you. }
\end{aligned}
$$

Odette looks mortified.

$$
\begin{gathered}
\text { ODETTE } \\
\text { Did she tell you? }
\end{gathered}
$$

June looks at her questioningly.

$$
\text { ODETTE }
$$

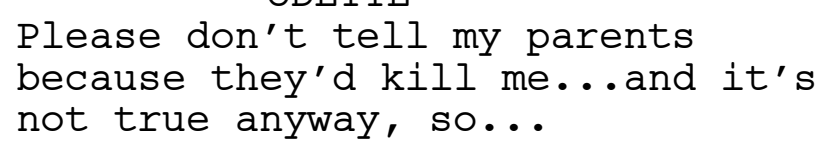

This is all the confirmation June needs. 
June slams the door on Odette, and turns to William, overcome with emotion.

William grimaces. He stands up, but instead of going to his wife he finally tends to the overflowing gravy.

$$
\begin{gathered}
\text { JUNE } \\
\text { I'm calling Doctor Owen. }
\end{gathered}
$$

EXT. MANNING HOUSEHOLD (1967) - NIGHT

Odette trudges down the driveway, stops at the road, and slumps down onto the ground.

INT. MANNING HOUSEHOLD, BATHROOM (1967) - NIGHT

Michael has lathered ointment all over his rash, and seems to have calmed down.

He heads back down the hallway.

INT. MANNING HOUSEHOLD, HALLWAY (1967) - NIGHT

Michael stops outside the door to the kitchen, which has been closed. He hears June sobbing, and puts his ear to the door.

INT. MANNING HOUSEHOLD, KITCHEN (1967) - NIGHT

June is on the phone, in tears.

William continues to stir the gravy in the background. JUNE (INTO PHONE)

And it will cure her?

June looks comforted by the response.

INT. HOWARD'S SHED (1999) - NIGHT

Bertha Jessop continues to scour through the files and clippings when she comes across a "wanted for murder" poster that has a picture of Bertha Manning in her twenties on it.

Bertha Jessop stares at it, stunned, when Howard appears in the doorway.

Bertha Jessop tries to hide the poster, but Howard has clearly seen it.

Bertha Jessop stands up, nervous and guilty.

Sorry

BERTHA JESSOP 
Howard smiles and shakes his head.

HOWARD

Your enthusiasm is encouraging.

Howard steps towards Bertha Jessop, and picks up the wanted poster.

HOWARD

Make any other discoveries?

Bertha Jessop looks bashful.

BERTHA JESSOP

I think there's a pattern to when

each Bertha found out about the

other Berthas. It goes from two

years apart, to four years apart,

to eight years apart.

Howard smiles and nods, like a proud father.

HOWARD

To sixteen years apart, to thirty

two years apart.

Bertha Jessop looks disappointed.

BERTHA JESSOP

You already know about that?

Howard laughs.

HOWARD

I've turned this thing inside-out

and upside-down.

BERTHA JESSOP

What do the numbers mean?

HOWARD

That you'll meet the next Bertha in

sixty four years.

Bertha Jessop hadn't thought of this, and is astonished.

Howard surveys her amazed expression.

Bertha Jessop's gaze falls on the wanted poster again. She fidgets, nervously.

BERTHA JESSOP

Did she really murder someone?

Howard discreetly places the wanted poster out of sight.

HOWARD

People's paths aren't always a

straight line, you know? 
Howard eyes Bertha Jessop submits and nods to pretend she understands.

Howard is unnerved by Bertha Jessop's uncertainty, so changes the subject.

\section{HOWARD}

I wasn't planning on showing you

this so early on, but I think it

might help you see how big this

thing really is.

Howard pulls out a box of casette tapes from under his workbench.

HOWARD

I haven't even played these to my wife.

He places one of the tapes in a cassette deck, and hits play. The tape crackles a little then plays Howard's recorded voice.

HOWARD (RECORDING)

Today's date is May 25th, 1998. It

is 12:02 pm. Based on recent

calculations I hypothesise that on

July 17th, 1999--the night after

Bertha Jessop will have arrived--I

will walk in on her searching

through my files.

Bertha Jessop is bewildered.

\section{HOWARD (RECORDING)}

She will discover the wanted poster with Bertha Manning's photo on it. She will apologise. I will forgive her. She will tell me she has uncovered the pattern of dates, and then will be disappointed when I tell her I am already aware of the pattern.

How. . ?

BERTHA JESSOP

Howard, proud, flashes her a wolfish grin. HOWARD (RECORDING)

She will then ask about the wanted poster, and I will distract her with this very recording.

Howard stops the cassette. 
BERTHA JESSOP

That's not possible.

HOWARD

Anything is possible, Bertha.

Bertha Jessop looks like she might vomit.

EXT. MANNING HOUSEHOLD (1967) - NIGHT

The house is dark and still, but the rain is pounding down causing flooding in the driveway.

INT. MANNING HOUSEHOLD, BERTHA'S BEDROOM (1967) - NIGHT

Bertha Manning is lying on her bed, flicking the locket of Bertha Greive open and shut over and over again.

There is a knock on the door.

Bertha Manning gets up and stands at the door.

$$
\begin{aligned}
& \text { BERTHA MANNING } \\
& \text { (whispering) } \\
& \text { Who is it? } \\
& \text { Michael. MICHAEL (O.S.) }
\end{aligned}
$$

Bertha Manning opens the door to let Michael in.

MICHAEL

We have to get out of here.

Bertha Manning looks skittish.

BERTHA MANNING

Father David said I have to stop

putting my needs before others.

MICHAEL

Your mum wants to lock you in a

loony bin.

Bertha tenses.

What?

\section{BERTHA MANNING}

MICHAEL

I heard her on the phone talking to

a doctor about you being queer, and

they're planning on electrocuting

you into not being queer anymore.

Bertha Manning can't believe what she's hearing. 
Queer?

BERTHA MANNING

Michael pulls out June's diary, and flicks to the page with tomorrow's date on it - July 25, 1967 - and shows it to Bertha Manning.

It has "Bertha's appointment with psychologist" written on it.

\title{
BERTHA MANNING
}

Bitch.

Michael smiles, pleased. He holds out the car keys to the Manning's car, suggestively.

Bertha Manning starts packs some things into a bag.

EXT. MANNING HOUSEHOLD (1967) - NIGHT

Michael and Bertha Manning make a mad dash for the car.

INT. MANNING CAR (1967) - NIGHT

Michael starts the car, does an erratic U-turn, then zooms down the flooded driveway.

They turn off seed Road and head down the hill when a figure runs out in front of the car and stands in the headlights.

\section{Jesus!}

MICHAEL

Michael brakes just in time.

Bertha Manning squints and her heart sinks as she recognises the figure - Odette, drenched from the rain and crying.

Odette approaches the side of the car. Michael winds down the window.

\section{Don't! \\ BERTHA MANNING}

Bertha tries to reach over to stop him, but she's too slow.

Odette grips onto the glass and peers in.

\author{
ODETTE \\ Where are you going? \\ BERTHA MANNING \\ None of your business.
}


ODETTE

Can I come with you? I need to talk

to you.

$$
\text { BERTHA MANNING }
$$

I hope you both burn in hell!

Odette looks crushed.

$$
\begin{aligned}
& \text { BERTHA MANNING } \\
& \text { Drive, Michael. }
\end{aligned}
$$

Michael hits the accelerator and hoons off, flinging Odette onto the muddy road.

EXT. ROAD ( 1967 ) - NIGHT

Odette runs down the rest of the hill after the black car.

EXT. COASTAL ROAD (1967) - NIGHT

Odette watches as the car zooms down the road that runs beneath the cliff, which is starting to crumble - gravel tumbling down the cliff-face towards the road.

Odette gives up, and trudges back into the township.

INT. MANNING CAR (1967) - NIGHT

Michael spots a van parked up on the road, and pulls over.

BERTHA MANNING

What're you doing?

MICHAEL

If we take that van, it'll be

harder for them to find us.

Michael hops out of the car, and Bertha Manning reluctantly follows.

EXT. COASTAL ROAD (1967) - NIGHT

More of the cliff starts to crumble, but Michael and Bertha Manning don't notice it in the pouring rain.

Michael rips the radio antenna off the Manning's car and they head towards the van.

Michael wiggles the antenna through the bottom of the van's window to unlock it.

They hop in and Michael uses the antennae to hot-wire the van's engine. 
They drive off, disappearing into the night.

Behind them, rocks tumble down the cliff which avalanche into a huge landslide, crushing the Manning car and bringing down Seed Road - including the Manning property - from above.

Nearing the town centre, Odette stops in her tracks and turns to see the destruction. Her eyes land on the crushed Manning car, and she lets out a gasping sob.

INT. VAN - NIGHT

Michael speeds up to escape the debris from the landslide, and once he's clear he brakes.

Michael and Bertha Manning turn to face the landslide.

$$
\text { MICHAEL }
$$

Holy shit.

Bertha Manning doesn't take her eyes off her house which has ripped in half, and continues to slide down the hill.

\section{MICHAEL}

We should go and help.

Bertha Manning turns back around, and stares up at the open road ahead.

$$
\text { Nah. }
$$

$$
\text { BERTHA MANNING }
$$

Michael's eyes go wide.

$$
\begin{aligned}
& \text { MICHAEL } \\
& \text { Are you sure? }
\end{aligned}
$$

Bertha Manning nods, assuredly.

Michael tentatively drives the van down the road.

BERTHA MANNING

I kinda like being immortal.

EXT. OPEN COUNTRY ROAD (1967) - NIGHT

The van hoons down the road.

INT. VAN (1967) - NIGHT

Bertha Manning stares out the window. Michael keeps glancing at her, disturbed at how calm and chipper she is.

Where are we heading? 


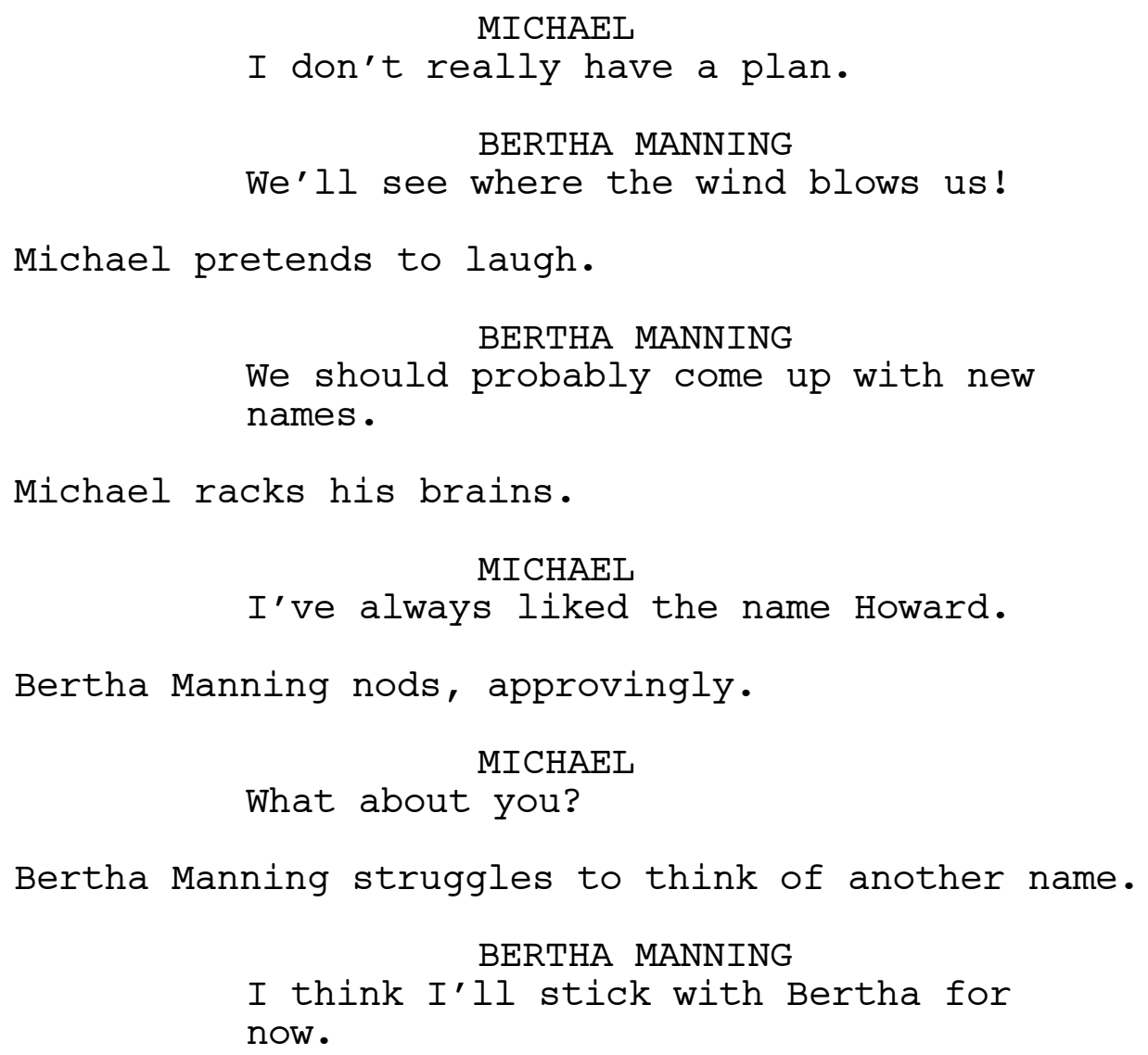

End of Episode. 


\section{THE CHANCES AND CHAOS}

The process of writing this serial was similar to that of Untold in that it started off as much more of an idea than it was a story. While I had written an outline of the serial and its characters for my PhD application, I was in no way committed or attached to what I had proposed. All that I was attached to was the idea that the serial would start with an unimaginable coincidence then go backwards in time to depict the series of events that led to the coincidence, and therefore suggest that the coincidence wasn't really a coincidence at all. I envisioned that the serial would reflect sensitive dependence to initial conditions, in that the it would end with the main event that started off the chain of events that led to the coincidence.

In order to achieve this, I knew I had to come up with a kind of coincidence that would be fascinating and believable enough for a fictional story, as well as a coincidence that could convincingly be the result of a series of individual events that were engaging in their own right. A type of coincidence that kept coming up in my research on strange coincidences, or perhaps one that I was drawn to, was that in which an individual would encounter someone who not only looked like them, but also had a lot of other strange similarities-same birthday, same name, same tastes, and often similar life experiences. And so, this was what I decided would be the focus of this particular serial-not just that a strange coincidence happens that might not be a coincidence, but to examine how people might respond differently to such uncanny occurrences. However, when I went to map the story out, I realised that if this was to happen to someone in real life, it would indeed be weird and uncanny, but it probably wouldn't be enough to construct an entire narrative around. So, I decided to open the narrative up by including a series of doppelgangers throughout history, rather than just two people. With this idea taking hold, I abandoned my desire to have the narrative depict how the coincidences came to be, and instead turned my focus onto how people who are near-identical, but from a different era and brought up in different environments, would react differently to learning about their doppelgangers. There would still be an element of explaining how the coincidences occur, but it was no longer the focus. 
The story I set out to tell in The Chances follows seven people throughout history who are all named Bertha, who all look alike, and who all learn about the Berthas before them at different ages and in different eras. Their varying reactions to the coincidence-which are based on their personal circumstances-determine the subsequent courses of their lives. Rather than having sensitive dependence on initial conditions frame a single narrative as I had initially proposed, I decided I would use the idea of sensitivity to initial conditions to frame several storylines, and-by placing each of the storylines alongside each other-show how changes in initial conditions can have drastically different results. In employing this method, my intention was to emphasise the way in which the narrative structures of serial dramas produce variety through repetition. And it was in the repetition that I thought I could establish the links and connections between each of the Berthas-in a way that allowed me to depict their individual stories, but also combine their stories to form a narrative whole.

This narrative whole is shaped by the successive depiction of each individual Bertha learning about the Berthas before them, but it is also shaped by and tied together with the overarching narrative of Bertha Jessop-the first Bertha we meet-who is held captive by Howard and Bertha Manning. In my first draft Bertha Jessop returned home after her strange encounters in Jamieson, and was forced to try to come to an understanding about the Berthas, and her own existence, alone. Changing the outcome of Bertha Jessop's narrative trajectory in the first episode allowed me to not only increase the stakes, but it also allowed for an ongoing narrative that depicts an investigation into the dramatic mystery of the chain of coincidences and ties all the other Bertha threads together. That is, the level of narrative closure of the other Bertha threads hinges on whether Howard and Bertha Jessop are able to find an answer or not, even though each of the Bertha threads is also distinct and separate from the others. Here we can see the way in which serial drama narratives are necessarily ongoing but also comprised of self-contained narratives, in the same way that the phase space curve that depicts of a system being pulled towards a strange attractor never directly intersects itself, but does repeatedly loop close to itself.

I also used a model of bifurcation as a way to order the various narrative threads. The first episode starts with the most recent Bertha-Bertha Jessop, in the 1990s, at age 13-and 
tells the story of her learning about the chain of Berthas before her. The second episode introduces the preceding Bertha-Bertha Manning, in the 1960s, at age 14-as she learns about the chain of Berthas prior to her, but also interweaves the continuing storyline of Bertha Jessop as a kind of ' $B$ ' story. The third episode focuses on the next Bertha-Bertha Greive, in the 1950s, at age 18-while interweaving the continuing storylines of Bertha Jessop and Bertha Manning. And so on, until the sixth and final episode which interweaves six Berthas, and focuses on two main storylines: Bertha Jessop in the future spotting her succeeding Bertha but deciding to break the cycle by not telling her, and the reveal that the original Bertha wasn't really a Bertha at all-she was just a strange lady who needed a friend so lied to a woman about having the same name and looking alike. I decided to have the narrative unfold as a set of bifurcations in an attempt to simultaneously emphasise the potential complexity of serial drama narratives but also minimise the potential for confusion. The bifurcations were a way to impose a kind of order and pattern on a narrative that might otherwise become sprawling and disorderly.

To take these bifurcations further, I made specific calculations based on a geometric sequence to determine how old each of the Berthas would be when their discovery of the chain of Berthas would take place. Each age was found by multiplying the previous age-gap by two. So, Bertha Jessop is 12, making Bertha Manning 14 (2 years difference), making Bertha Greive 18 (4 years difference), making the next Bertha 26 ( 8 years difference) and so on. And the years in which each Bertha discovers the coincidence were established with the same sequence, but in reverse, so that as the age gap between each pair of Berthas gets further apart, the closer together the years in which the coincidence is revealed to them become-Bertha Jessop finds out in 1999, Bertha Manning finds out in 1967 (32 years apart), Bertha Greive finds out in 1951 (16 years apart) etc. While these calculations have no explicit bearing on the narrative or how the events might be interpreted by the characters or the audience, they at least provided an order for me as a writer. Just like the conventions and formulae of TV drama narratives in general, the constraints of this method sparked my creativity; rather than plucking out random numbers from thin air and basing the characters and their lives on these arbitrary numbers, I was able to develop the characters and their lives based on something more tangible and controlled. 
Using this model of bifurcation to shape the details of this TV narrative meant that I was also implementing the fractal-like structures of serial dramas by introducing a new Bertha in each episode. That is, while each Bertha has a different reaction, the shape of their narrative trajectory is essentially the same and they fold in on each other. For example, Bertha Jessop is living her normal, miserable life when she learns about the chain of Berthas and is excited by it at first because, to her, it represents the "family unit" that she so desperately wants. But after living with the new "family unit", she starts to question the validity of the strange occurrence, which sends her down a path of paranoia and anxiety that she may not have otherwise experienced. In a similar, but different, way, when Bertha Manning-who has been brought up to think she is the centre of the universe-finds out about the doppelgangers she sees it as a confirmation of what she already knew. But when things start happening to her that prove she is not the centre of the universe she starts to view the coincidence in a negative light, leading her down a dark path that she may not have otherwise taken. Thus, as well as each self-contained narrative trajectory reflecting each other, these trajectories also form a continuous, ongoing narrative in the way that I discussed in relation to strange attractors. The trajectories are similar, but they also diverge in a way that forms the shape of the serial as a whole.

The process of writing The Chances, as well as Untold and Bad News, has elucidated the fact that randomness in serial drama narratives can't be pure randomness, even if it's trying to appear random or deal with randomness in the narrative itself. There has to be an element of design, determinism, or order underpinning any disorder in the narrative (and perhaps even underpinning the disorder of the writing process)-whether it's visible or not. This obviously reflects the idea that is at the heart of chaos-that a system may appear unstable and disorderly, but it has underlying structures that are determining its behaviour. Writing each of these TV projects with chaos in mind hasn't necessarily resulted in the most organic or natural writing process-and I am in no way arguing for all TV writers to employ a chaos framework-but I do think that using such a specific and involved method has elucidated how and why the serial drama is often referred to as a complex narrative form. Its complexity isn't always in the types of stories it tells, it's in how these stories are told, and in ensuring that the "how" isn't always obvious or predictable to a viewer. 


\section{CONCLUSION}

In my introduction I suggested that the Lorenz Waterwheel is an apt analogy for the interconnected components of TV serial drama narratives. After examining the simple and repeated narrative structures that underpin all serial dramas through the lens of "Universality" in Chapter One, and then-in Chapter Two-using strange attractors to examine how these simple and repeated narrative structures are used to produce unpredictable, yet expected, storylines, we can understand this analogy of the Lorenz Waterwheel in more depth. We can think of the premise of a successful serial drama as being like the flow of water going into the Lorenz Waterwheel; the speed at which water flows, or the intended execution of a show's premise, determines how fast or slow the buckets will fill up. The buckets are like the various parts and units that comprise a serial drama narrative, which will be utilised in different ways depending on the execution of a serial's tone, style and texture. The water leaking out of the holes at the bottom of each bucket can be likened to the plot of a TV serial, which is made up of the main characters, their goals and desires, how they go about achieving these, and the obstacles that stand in their way. In the same way that the leaking water determines the motion of the waterwheel, which in turn determines how much each bucket then fills up and so on, the plot of a serial drama determines and is determined by the show's premise and the way in which the underlying structural units and parts are used. All of the interlinked components of the Lorenz Waterwheel work together in a way that produces unpredictable motion, just as all the interlinked components of a successful serial drama produce an unpredictable story.

Examining the various components of serial drama narratives in this way, through the lens of chaos, helps elucidate the interdependence of complexity and simplicity in successful serial dramas. From my perspective as a writer, serial dramas are complex because of the intricate way their narratives are built off and embedded with simple and repeated structures and patterns. Chaotic systems are deemed chaotic not because they display pure randomness and disorder, but because of the intricate intertwining of order and disorder, 
regularity and irregularity. In the same way, serial drama narratives are complex not because they are erratic and disordered, but because of the underlying structures and formulae that determine the expected-yet-unpredictable shape of the serial. The repeated narrative structures of serial dramas, like the buckets of the Lorenz Waterwheel, function in a simple and expected way, but when examining these simple structures in terms of the serial, or "system", as a whole, we can see that they are not only used to produce complex and unpredictable storylines, they are the reason that serial dramas are complex and unpredictable. The complexity of TV serial dramas is inherently simple, which means that the simplicity of a serial drama is also inherently complex.

Applying my analysis of serial drama narratives through the lens of chaos to the structure and content of my own three TV projects has also helped expound the nature of complexity in serial drama narratives. Through my critical component I was not only able to demonstrate the similarities between chaotic systems and serial drama narratives that I had discussed in my critical component, but I was also able to-as a beginning writer-reinforce and enrich my own understanding of how the structures and rules of serial dramas work. With this deeper understanding I have been able to generate three unique and ambitious TV projects that I might not otherwise have attempted to write. Just as the constraints of the narrative form enable TV serials to flourish, I have used the models of fractals, strange attractors, and sensitive dependence on initial conditions to form a specific kind of constraint that has opened up my writing process. When I started this thesis three years ago I had a tendency to delete and edit my words and ideas before even putting them down on the page for fear of them being too simple and obvious, quashing the potential for those ideas to turn into something more substantial. Writing these three TV projects through the lens of chaos has allowed me to experiment with the fundamental mechanics of storytelling in TV drama and embrace the often overwhelming and clumsy process of TV writing. While the process has proven difficult at times-due to my tendency to prioritise a complex premise or idea over constructing a digestible and engaging story-the process allowed me to reflect on why particular elements in the narrative weren't working, or why I was having such a difficult time writing a particular aspect of the narrative. The answer to these questions, more often than not, was that I was missing something fundamental or simple in the story (a character goal, what was at stake, or how a storyline was meant to fit into the 
overarching storyline). From these realisations and discoveries I have been able to deduce that there is a correlation between the interdependent relationship of simplicity and complexity in serial drama narratives and the interdependent relationship of story and structure in serial drama narratives. In each case, one cannot exist without the other.

In writing these three serials through the lens of chaos I have been able to establish and solidify the narrative bounds and foundations for each of my serials, which I can now use to guide me in the next stages of developing and refining the narratives. In other words, using chaos has strengthened my grasp on how to implement and embrace simplicity in order to develop more engaging storylines that will eventually, after subsequent drafts, become more seamlessly complex. In this sense, the process of writing itself can be likened to a chaotic system - I have learnt the importance of experimenting with the narrative because something as small as a line of dialogue could end up informing the entire shape of a serial. I believe this thesis could provide a useful guide for other beginning writers to understand the power and significance of the simple underlying patterns and structures that are necessary for the success of any serial drama.

My approach of using chaos to examine serial drama narratives is especially relevant at a time when the landscape of TV is ever-changing, as TV writers continue to push the boundaries and challenge the conventions of TV drama narratives, and as the viewing habits of TV audiences are shifting (such as the move from watching episodes weekly to "binge watching" an entire serial in one sitting). And, while my thesis has focused on the complexity of serial dramas in terms of their narrative components and form, further aspects that I haven't included in this thesis, but could also be examined through the lens of chaos, are the conditions of TV writing. That is, external factors and variables (which include such things as the collaborative dynamic of TV writing rooms, budget, ratings, production constraints and limitations, writers' strikes, and actors' availability) also have a big impact on determining the development and shape of a serial drama. Just as the premise and execution of a serial can be likened to the water that flows into the Lorenz Waterwheel and determines the system's unpredictable motion, there are also several external variables that can push and pull a TV drama in unpredictable directions. For example, if an actor in a TV show drops out for whatever reason, then the writers of that serial will have to build the 
actor's character's departure into the script somehow, which will then (because of how interlinked the components of a TV serial are) have a big impact on how future storylines will play out. While these external factors are largely uncontrollable, their effects have to be managed in a way that sustains and feeds into the bounds of the serial drama that are set up at the beginning. In terms of my own TV projects, the narratives could have gone in completely different directions had they been worked on in a writers' room, like most TV shows are, where several different people with different ideas and perspectives would be determining the direction and shape of the story. Yet the essence of each TV project would have remained the same.

Ultimately, all the components of a TV serial drama-whether they exist inside or outside of the narrative-have to come together to achieve the most important aspect of a serial-to keep its viewers engaged and entertained. As Ellen Sandler writes in relation to how to turn an idea into a story, "[i]f it sounds simple, it's because it is. Simple is what we are going for. But as we also know, simple is not easy" (72). Indeed, the simplicity of TV serial dramas is not easy. It's not easy because its simplicity goes hand-in-hand with its complexity. It is through this interdependent relationship between simplicity and complexity that a serial drama is able to be rigidly structured yet endlessly creative; familiar and expected yet surprising and unpredictable; the same but different. 


\section{BIBLIOGRAPHY}

Allen, Robert C., Channels of Discourse, Reassembled: Television and Contemporary Criticism, London, Routledge, 2000.

Allrath, Gaby and Marion Gymnich, editors. Narrative Strategies in Television Series. New York: Palgrave Macmillan, 2005.

Armbrust, Sebastian. "House vs The Wire: Procedure and Complexity in Contemporary US Serial Television Drama." Diegesis: Interdisciplinary E-Journal for Narrative Research, vol. 2, no. 1, 2013, pp. 1-22. DIEGESIS, www.diegesis.uniwuppertal.de/index.php/diegesis/article/view/122/145.

Boeing, Geoff. "Visual Analysis of Nonlinear Dynamical Systems: Chaos, Fractals, SelfSimilarity and the Limits of Prediction." Systems vol. 4, no. 4, 2016 ProQuest, doi:10.3390/systems4040037.

Berg, Peter, creator. Friday Night Lights. NBC, 2006.

Bordwell, David. Narration in the Fiction Film. Madison, University of Wisconsin Press, 1985. Bouquiaux, Laurence. "Monads and Chaos: The Vitality of Leibniz's Philosophy." Diogenes vol. 41, no. 161, 1993, pp. 87-105. Sage, doi.org/10.1177/039219219304116107.

Butler, J. G. Television: Critical Methods and Applications. New Jersey, Lawrence Erlbaum Associates, 2002.

Brady, Patrick., "Chaos Theory, Control Theory, and Literary Theory or: A Story of Three Butterflies." Modern Language Studies, vol. 20, no. 4, 1990, pp 65-79. Jstor, DOI: $10.2307 / 3195061$.

Boeing, Geoff. "Visual Analysis of Nonlinear Dynamical Systems: Chaos, Fractals, SelfSimilarity and the Limits of Prediction." Systems vol. 4, no. 4, 2016 ProQuest, doi:10.3390/systems4040037.

Chase, David, creator. The Sopranos. HBO, 1999.

Coleman, David, et al. "Finding Meaning in All the Right Places: A Novel Measurement of Dramatic Structure in Film and Television Narratives." Projections, vol. 7, no. 2, 2013, pp. 92-110. Gale, DOI: 10.3167/proj.2013.070205.

Collins, Jim. "Television and Postmodernism." Channels of Discourse, Reassembled, edited by Robert C. Allen. 2nd ed, U of North Carolina P, 1992, pp. 327-353. 
Creeber, Glen. Serial Television: Big Drama on the Small Screen. London, BFI Publishing, 2004.

DiMaggio, Madeline. How to Write for Television. New York, Fireside, 2008.

Dunleavy, Trisha. Television Drama: Form, Agency, Innovation. New York: Palgrave Macmillan, 2009.

----. Complex Serial Drama and Multiplatform Television. New York: Routledge, 2018.

Eigenauer, John D., "The Humanities and Chaos Theory: A Response to Steenbug's 'Chaos at the Marriage of Heaven and Hell'". The Harvard Theological Review, vol. 86, no. 4, 1993, pp. 455-469. Jstor, doi.org/10.1017/S0017816000030662.

Epstein, Alex. Crafty TV Writing: Thinking Inside the Box. New York, Owls Books, 2006.

Feigenbaum, Mitchell J. "Universal Behavior in Nonlinear Systems.” Physica D: Nonlinear Phenomena, vol. 7, no. 1, 1983, pp. 16-39. ScienceDirect, doi.org/10.1016/01672789(83)90112-4.

Feuer, Jane. "Narrative Form in American Network Television." High Theory/Low Culture, edited by Colin MacCabe, Manchester UP, 1986, pp.101-14.

Fiske, John \& John Hartley. Reading Television. London: Routledge, 2003.

Franklin, Nancy. "The Heart of Texas: 'Friday Night Lights'.” The New Yorker, 8 Oct. 2007, newyorker.com/magazine/2007/10/08/the-heart-of-texas. Accessed 4 April, 2017.

Gaddis, John Lewis. The Landscape of History: How Historians Map the Past. New York, Oxford University Press, 2002.

Galatzer-Levy, Robert M. “Finding Your Way Through Chaos, Fractals, and Other Exotic Mathematical Objects: a Guide for the Perplexed." Journal of the American Psychoanalytic Association, vol. 57, no. 5, 2009, pp. 1227-1249. Sage, doi.org/10.1177/0003065109347905.

Gil, Steven John. "'Remember the Nox': Stargate: SG-1 's Narrative Structure and the Changing Form of Television Fiction." New Review of Film and Television Studies, 2014, pp. 1-25. Taylor \& Francis, DOI: 10.1080/17400309.2014.880643.

Gilligan, Vince, creator. Breaking Bad. AMC, 2008.

Gleick, J. Chaos: The Amazing Science of the Unpredictable. London, Vintage, 1998.

Goldberg, Lee and William Rabkin. Successful Television Writing. Hoboken, New Jersey, John Wiley \& Sons, 2003. 
Goldstein, Jeffrey. "Chaos, Constants and Insensitivity to Initial Conditions: an Introduction to Mitchell Feigenbaum's 'Universal Behavior in Nonlinear Systems'.” Vol. 17, no. 3, 2015, Academic OneFile, 10.emerg/10.17357.86372e6b08fec2ef73370772c7fc27e9.

Gregersen, Hal, and Lee Sailer. "Chaos Theory and Its Implications for Social Science Research." Human Relations, vol. 46, no. 7, 1993, pp. 777-802. Sage, DOI: 10.1177/001872679304600701.

Hayles, Katherine, N., editor. Chaos and Order: Complex Dynamics in Literature and Science. The University of Chicago Press, 1991.

Hirsh, Marc. "Plot twist will ruin 'Friday Night Lights'." today.com, 11 Oct. 2007, today.com/popculture/plot-twist-will-ruin-friday-night-lights-wbna21230604.

Hodge, Bob. "Life, Chaos, and Transdisciplinarity: A Personal Journey." World Futures, vol. 63, no. 3-4, 2007, pp. 209-222. Francis \& Taylor, DOI: 10.1080/02604020601172558.

Kasey, Gerald. Writing for Television. London, A \& C Black, 1999.

Kellert, Stephen H. "Science and Literature and Philosophy: the Case of Chaos Theory and Deconstruction." Configurations, vol. 4, no. 2, 1996, pp. 215-232. Project MUSE, doi:10.1353/con.1996.0013.

Kirby, David A. “Forensic Fictions: Science, Television Production, and Modern Storytelling." Studies in History and Philosophy of Biol \& Biomed Sci, vol. 44, no. 1, 2013, pp. 92-102. ScienceDirect, doi.org/10.1016/j.shpsc.2012.09.007.

Kozloff, Sarah. "Narrative Theory and Television." Channels of Discourse, Reassembled, edited by Robert C. Allen, 2nd ed, U of North Carolina P, 1992, pp.61-100.

Lindelof, Damon, Jeffrey Lieber and J.J. Abrams, creators. Lost. ABC, 2004.

Lindelof, Damon and Tom Perrotta, creators. The Leftovers. HBO, 2014.

Lorenz, Edward. The Essence of Chaos. London, UCL Press, 1995.

Mackenzie, Adrian. "The Problem of the Attractor: A Singular Generality between Sciences and Social Theory." Theory, Culture \& Society, vol. 22, no. 5, 2005, pp. 45-65. Sage, DOI: $10.1177 / 0263276405057190$.

Marion, Russ. The Edge of Organization: Chaos and Complexity Theories of Formal Social Systems. Thousand Oaks, Sage, 1999.

Mandelbrot, B. "How Long Is the Coast of Britain? Statistical Self-Similarity and Fractional Dimension." Science (New York, N.Y.), vol. 156, no. 3775, 1967, pp. 636-8. AAAS, DOI: 
10.1126/science.156.3775.636.

Miller, Kristen. "From Fears of Entropy to Comfort in Chaos: Arcadia, The Waste Land, Numb3rs, and Man's Relationship with Science." Bulletin of Science, Technology and Society, vol. 27, no. 1, 2007, pp. 81-94. Sage, DOI: 10.1177/0270467606297626.

Mittell, Jason. Complex TV: The Poetics of Contemporary Television Storytelling. New York, New York University Press, 2015.

----. "All in the Game: The Wire, Serial Storytelling and Procedural Logic." Third Person Authoring and Exploring Vast Narratives, edited by Pat Harrigan and Noah WardripFruin, MIT, 2009, pp.429-439.

----. "Lost in a Great Story: Evaluation in Narrative Television (and Television Studies)." Reading LOST: Perspective on a Hit Television Show, edited by Roberta Pearson, Tauris, 2009, pp. 119-38.

----. “Narrative Complexity in Contemporary American Television." Matrizes, vol. 5, no. 2, 2012, pp. 29-52. ebscohost, DOI: http://dx.doi.org/10.11606/issn.19828160.v5i2p29-52.

Morreale, Joanne. "Lost, The Prisoner, and the End of the Story." Journal Of Popular Film And Television, vol. 38, no. 4, 2010, pp. 176-185. ProQuest, DOI: 10.1080/01956051.2010.508504.

Nannicelli, Ted. "It's all Connected: Televisual Narrative Complexity." The Wire: Urban Decay and American Television, edited by Tiffany Potter and C.W. Marshall, Bloomsbury Academic \& Professional, 2015, pp. 190-202. ProQuest Ebook Central, ebookcentral.proquest.com/lib/vuw/detail.action?doclD=564206.

Ndalianis, A. "Television and the Neo-Baroque." The Contemporary Television Serial, edited by Lucy Mazdon and Michael Hammond, Edinburgh University Press, 2005, pp. 83101. Jstor, www.jstor.org.helicon.vuw.ac.nz/stable/10.3366/j.ctt1r29hq.

Nelson, R., TV Drama: 'Flexi-Narrative Form', and 'a New Affective Order', 8th Annual Conference: German Society for Contemporary Theatre and Drama in English, 1999, Schloss Rauischholzhausen, Wissenschaftlicher Verlag Trier, 2000.

Newman, Michael. "From Beats to Arcs: toward a Poetics of Television Narrative." Velvet Light Trap, no. 58, 2006, pp. 16-28. ProQuest, www.muse.jhu.edu.helicon.vuw.ac.nz/journals/the_velvet_light_trap/v058/58.1new man.html. 
O'Neal, Sean. "Here's the Lost episode Carlton Cuse and Damon Lindelof Think is the Absolute Worst." AV Club, 8 May, 2014, www.news.avclub.com/heres-the-lostepisode-carlton-cuse-and-damon-lindelof-1798268417. Accessed 1 December 2017.

Outlaw, Kofi. "Lost Finale Explained: Answering the Unanswered Questions." Screenrant, 24 May, 2010, www.screenrant.com/lost-finale-explanation/.

Parker, Jo Alyson. Narrative Form and Chaos Theory in Sterne, Proust, Woolf, and Faulkner. Basingstoke, Palgrave Macmillan, 2007.

Polvinen, Merja. "The Ends of Metaphor." European Journal of English Studies, vol. 11, no. 3, 2007, pp. 273-284. Taylor \& Francis, DOI: 10.1080/13825570701409581.

Porter, Michael J., et al. "Re(De)Fining Narrative Events: Examining Television Narrative Structure." Journal of Popular Film and Television, vol. 30, no. 1, 2002, pp. 23-30. ProQuest, DOI: 10.1080/01956050209605556.

Raisio, Harri, and Niklas Lundström. "Managing Chaos." Administration \& Society, vol. 49, no. 2, 2017, pp. 296-315. Sage, DOI: 10.1177/0095399714541269. 3 January 2016.

Rincón, Omar. “Nuevas Narrativas Televisivas: Relajar, Entretener, Contar, Ciudadanizar, Experimentar/New Television Narratives: Entertainment, Telling, Citizenship, Experimental." Comunicar, vol. 18, no. 36, 2011, pp. 43-50. ProQuest, DOI: 10.3916/C36-2011-02-04

Sandler, Ellen. The TV Writer's Workbook: A Creative Approach to Television Scripts. New York, Bantam Dell, 2007.

Sconce, Jeffrey. "What If? Charting Television's New Textual Boundaries." Television after TV: Essays on a Medium in Transition, edited by Lynn Spigel and Jan Olsson, Duke UP, 2004, pp.93-112.

Simon, David, creator. The Wire. HBO, 2002.

Slethaug, Gordon E. Beautiful Chaos: Chaos Theory and Metachaotics in Recent American Fiction, State University of New York Press, 2000. ProQuest Ebook Central, ebookcentral.proquest.com/lib/vuw/detail.action?doclD=3408029.

Stewart, lan. Does God Play Dice?: The Mathematics of Chaos. Cambridge, Mass, Basil Blackwell, 1990.

Thompson, Kristin. Storytelling in Film and Television. Cambridge, MA/London, Harvard University Press, 2003. 
VanDerWerff, Todd. "The Delicate Art of the TV Series Finale." Vox, 17 Oct, 2017, http://vox.com/culture/2017/10/17/16462246/series-finale-best-worst-tv-halt-andcatch-fire-americans. Accessed 1 December 2017.

Vees-Gulani, Susanne. "'Hidden Order in the Stoppard Set': Chaos Theory in the Content and Structure of Tom Stoppard's Arcadia." Modern Drama, vol. 42, no. 3, 1999, pp. 411-426. ProQuest, DOI: 10.3138/md.42.3.411.

Williams, Linda. On the Wire, Duke University Press, 2014. ProQuest Ebook Central, ebookcentral.proquest.com/lib/vuw/detail.action?doclD=1757308. 